\title{
Dynamics Modeling and Simulation of Flexible Airships
}

\author{
by \\ Yuwen Li \\ Department of Mechanical Engineering \\ McGill University, Montreal
}

Canada

January 2008

A thesis submitted to McGill University

in partial fulfillment of the requirements of the degree of

Doctor of Philosophy

(C) Yuwen Li, 2008 


\section{Abstract}

The resurgence of airships has created a need for dynamics models and simulation capabilities of these lighter-than-air vehicles. The focus of this thesis is a theoretical framework that integrates the flight dynamics, structural dynamics, aerostatics and aerodynamics of flexible airships.

The study begins with a dynamics model based on a rigid-body assumption. A comprehensive computation of aerodynamic effects is presented, where the aerodynamic forces and moments are categorized into various terms based on different physical effects. A series of prediction approaches for different aerodynamic effects are unified and applied to airships. The numerical results of aerodynamic derivatives and the simulated responses to control surface deflection inputs are verified by comparing to existing wind-tunnel and flight test data.

With the validated aerodynamics and rigid-body modeling, the equations of motion of an elastic airship are derived by the Lagrangian formulation. The airship is modeled as a free-free Euler-Bernoulli beam and the bending deformations are represented by shape functions chosen as the free-free normal modes. In order to capture the coupling between the aerodynamic forces and the structural elasticity, local velocity on the deformed vehicle is used in the computation of aerodynamic forces. Finally, with the inertial, gravity, aerostatic and control forces incorporated, the dynamics model of a flexible airship is represented by a single set of nonlinear ordinary differential equations.

The proposed model is implemented as a dynamics simulation program to ana- 
lyze the dynamics characteristics of the Skyship-500 airship. Simulation results are presented to demonstrate the influence of structural deformation on the aerodynamic forces and the dynamics behavior of the airship. The nonlinear equations of motion are linearized numerically for the purpose of frequency domain analysis and for aeroelastic stability analysis. The results from the latter for the Skyship-500 airship indicate that this vehicle is not susceptible to aeroelastic instability in its operating range. However, these problems may arise for modern airship designs with thin film materials. 


\section{Résumé}

L'intérêt renouvelé envers les dirigeables a créé un besoin de modèles dynamique et de simulations de ces véhicules plus légers que l'air. Cette thèse traite d'un cadre théorique qui intègre la dynamique de vol, la dynamique structurale, l'aérostatique et l'aérodynamique des dirigeables flexibles.

La recherche débute par une étude d'un modèle dynamique fondé sur l'hypothèse d'un corps rigide. Une approche de calcul d'aérodynamique complète est présentée, où les forces et les moments aérodynamiques sont classés par catégories basées sur différents effets physiques. Une série d'approches de prédiction des différents effets aérodynamiques est unifiée et appliqué aux dirigeables. Les résultats numériques des dérivés aérodynamiques et des réponses simulées à des commandes spécifiés sont comparés à des résultats d'essais provenant d'autre œuvres.

Une fois l'aérodynamique et le modèle de corps rigide validés, les équations de mouvement d'un dirigeable élastique sont dérivées avec une formulation Lagrangienne. Le dirigeable est modélisé comme poutre Euler-Bernoulli et les déformations sont représentées par des fonctions de forme choisies. Afin de prendre en considération la dépendance entre les forces aérodynamiques et l'élasticité structurale, la vitesse locale sur le véhicule déformé est employée dans le calcul des forces aérodynamiques. En conclusion, avec les forces d'inertie, de gravité, d'aérodynamique et de commande incorporées, le modèle dynamique d'un dirigeable flexible est exprimé sous la forme d'un ensemble d'équations différentielles ordinaires non-linéaires.

Le modèle proposé est mis en pratique sous forme de simulation dynamique afin 
d'analyser les caractéristiques dynamiques du dirigeable Skyship-500. Des résultats de simulation sont présentés pour démontrer l'influence de la déformation structurale sur les forces aérodynamiques et le comportement dynamique du dirigeable. Les équations non-linéaires de mouvement sont linéarisées numériquement pour permettre une analyse dans le domaine de fréquences, ainsi qu'une analyse de la stabilité aéroélastique. Les résultats indiquent que le véhicule n'est pas susceptible d'être instable aéroélastiquement dans ses conditions d'opérations normales. Cependant, ces problèmes peuvent surgir dans le cas de dirigeables modernes construits avec matériaux plus minces. 


\section{Acknowledgements}

There are many people I would like to thank, without whom this thesis would never have been completed. First, I would like to express my deep gratitude to my research supervisors, Professor Meyer Nahon and Professor Inna Sharf, for their guidance, encouragement and support over the past years. I want to thank them for their critical reading of the thesis draft and all the technical documents I have written during the course of this work. I have benefited immensely from their comments, remarks, suggestions, and their broad knowledge on the various related disciplines.

My sincere thanks also go to Professor Stuart J. Price for his two courses that helped me enter the fields of flight dynamics and aeroelasticity.

I want to thank my fellow students at CIM who have created a pleasant atmosphere for research including Phillippe Coulombe-Pontbriand, Alistair Howard, Jie Li, Lianzhen Luo, Zhongjing Ma, Marco Masciola, Gabriel Meunier, Jonathan Miller, Zhi Qi, Shivakumar Ranganathan, Jianfeng Yin. Special thanks to Dr. Casey Lambert, who was always willing to help. I am grateful to Nicolas Plamondon for his kind help in correcting the thesis abstract in French.

I am also pleased to acknowledge Jan Binder, Cynthia Davidson and Joyce Nault for their administrative assistance.

Finally, I thank my wife Shuhua and my family: my dad, my mom, my brother and my sister. Words would never be enough to express my gratitude to their understanding, support and love. 


\section{Claim of Originality}

Following is a summary of the main contributions of this thesis:

- A clear and physically justified development and implementation of the dynamics model of flexible airships. This is the first work to present an integrated treatment of the flight dynamics, aerostatics, aerodynamics and structural dynamics for LTA aircraft.

- A comprehensive aerodynamics computational method for flexible airships. A series of prediction approaches for various aerodynamic effects are unified and applied to airships. Particularly, a formulation of the potential-flow aerodynamic forces and moments are provided for flexible maneuvering airships. The influence of structural deformation of the airship hull has been incorporated. The method is effective and can be used in a dynamics simulation program.

- A validation of the aerodynamics and dynamics models by using existing flight test results.

- Generation of simulation results that demonstrate the impact of aerodynamics on the structural vibration and the effects of flexibility on the aerodynamics and transient response of elastic airships.

- A prediction method of the aeroelastic instability condition of flexible airships. This is the first theoretical work on the aeroelastic stability analysis of airships. 


\section{Contents}

$\begin{array}{ll}\text { Abstract } & \text { i }\end{array}$

Résumé $\quad$ iii

$\begin{array}{lll}\text { Acknowledgements } & \text { V }\end{array}$

Claim of Originality vi vi

List of Figures $\quad$ xi

List of Tables $\quad$ xiv

Nomenclature $\quad$ XV

1 Introduction 1

1.1 Background ............................ 1

1.2 Literature Review . . . . . . . . . . . . . . . . . 3

1.2.1 Dynamics models of airships . . . . . . . . . . . . . . 3

1.2.2 Structural analysis of airships and related inflated structures . . 9

1.2.3 Dynamics of flexible HTA aircraft . . . . . . . . . . . . . 12

1.2.4 Vibration of ships . . . . . . . . . . . . . . 14

1.3 Thesis Objectives and Organization . . . . . . . . . . . 15

1.4 Note on Notation . . . . . . . . . . . . . . . . . . 16 
2.1 Equations of Motion for Airship in Vacuum . . . . . . . . . . . . . 18

2.2 Interaction Forces and Moments Between Airship and Air: Aerostatics 21

2.3 Interaction Forces and Moments Between Airship and Air: Aerodynamics 22

2.3.1 Added-mass force and moment . . . . . . . . . . . . . 23

2.3.2 Viscous effect on the hull, normal to the centerline . . . . . . 28

2.3.3 Force acting on the fins, normal to the centerline . . . . . . . . 29

2.3.4 Force acting on the hull due to the fins, normal to the centerline 32

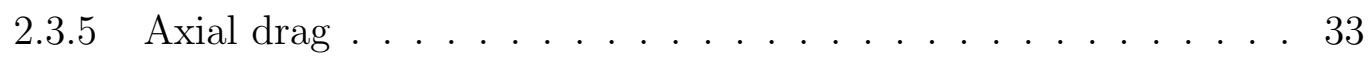

2.3.6 Force and moment due to control surface deflection . . . . . . . 33

2.4 Linearized Model . . . . . . . . . . . . . . . . . . . . 35

2.5 Numerical Simulation . . . . . . . . . . . . . . . . . . . . 37

2.5.1 Validation of the aerodynamics model . . . . . . . . . . 37

2.5.2 Nonlinear dynamics simulation for the Skyship-500 airship . . . 39

2.5.3 Linearized model and stability analysis for the Skyship-500 airship 45

2.6 2-DOF Model for Steady Turns . . . . . . . . . . . . . 51

2.6.1 Simplified model . . . . . . . . . . . . . 53

2.6.2 Comparison to the 6-DOF model . . . . . . . . . . . 55

3 Dynamics Model of Flexible Airships: Equations of Motion 58

3.1 Kinematic Description ................... . 59

3.1.1 Shape functions . . . . . . . . . . . . . 60

3.2 Equations of Motion . . . . . . . . . . . . . . . . . . . 62

3.3 Inertial Terms . . . . . . . . . . . . . . . . . . . 64

3.3.1 Linear and angular momenta. . . . . . . . . . . 64

3.3.2 Mass matrix and inertial force ............. 65

3.4 Gravity, Aerostatic and Thrust Forces . . . . . . . . . . . . 68 
4.1 Velocity Computation for Aerodynamics . . . . . . . . . . . . 72

4.2 Potential-flow aerodynamic force $\ldots \ldots \ldots \ldots \ldots$

4.2.1 Boundary condition of the Laplace equation . . . . . . . . . 74

4.2.2 Fluid kinetic energy and added-mass matrix . . . . . . . 76

4.2.3 Potential-flow aerodynamic force expressions . . . . . . . . 78

4.2.4 Computation of the added-mass matrix . . . . . . . . . . 81

4.3 Viscous Effect on the Hull . . . . . . . . . . . . . . . . . . 85

4.4 Force Acting on the Fins . . . . . . . . . . . . . . . 87

4.5 Force Acting on the Hull due to the Fins $\ldots \ldots \ldots . \ldots . \ldots 8$

$4.6 \quad$ Axial Drag . . . . . . . . . . . . . . . . . . . . . . . . 990

4.7 Force and Moment due to Control Surface Deflection . . . . . . . . . 91

\section{Dynamics Model of Flexible Airships: Implementation and Simula-} $\begin{array}{ll}\text { tion } & 92\end{array}$

5.1 Implementation $\ldots \ldots \ldots \ldots \ldots \ldots \ldots \ldots$

5.1.1 Summary of the dynamics model . . . . . . . . . . . . . 93

5.1 .2 Implementation . . . . . . . . . . . . . . . . . . 95

5.1 .3 Validation . . . . . . . . . . . . . . . . . . 95

$5.2 \quad$ Simulated Airship . . . . . . . . . . . . . . . . . . . . 97

5.3 Shape Functions and Some Shape Function Integrals _ . . . . . . 98

5.3 .1 Shape functions . . . . . . . . . . . . . . . . 98

5.3 .2 Shape function integrals . . . . . . . . . . . . . . . . . . 99

5.4 Time Responses . . . . . . . . . . . . . . . . . . . . . . . . . 102

5.4 .1 Case I: Response to elevator input . . . . . . . . . . . . . 102

5.4 .2 Case II: Response to rudder input . . . . . . . . . . . . . . 103

5.4 .3 Case III: Path-following flights . . . . . . . . . . . . . . 105

5.5 Frequency Response . . . . . . . . . . . . . . . . . . . . . . 109 
6 Dynamics Model of Flexible Airships: Aeroelastic Stability

6.1 Aeroelastic Instability . . . . . . . . . . . . . . . . . 116

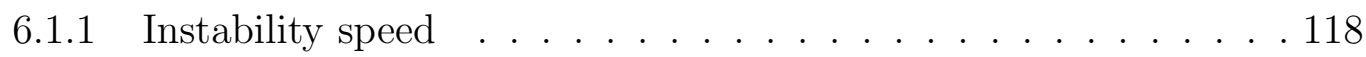

6.1 .2 Time response at high speeds . . . . . . . . . . . . 125

6.2 Influence of $E T$ on $u_{\text {unstable }} \ldots \ldots \ldots \ldots \ldots \ldots \ldots \ldots \ldots \ldots$

6.3 Summary of the Aeroelastic Stability Analysis . . . . . . . . . . . 131

7 Conclusions 132

7.1 Recommendations for Future Research . . . . . . . . . . . . . . 135

$\begin{array}{ll}\text { Bibliography } & 137\end{array}$ 


\section{List of Figures}

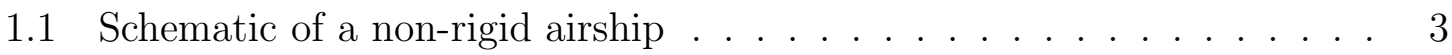

1.2 Hybrid heavy lift airship in $[13] \ldots \ldots \ldots \ldots \ldots$

1.3 The Skyship-500 airship . . . . . . . . . . . . . . . 5

1.4 The High Platform II airship . . . . . . . . . . . . . . . . . . . . 11

1.5 Schematic of aeroelasticity $[89] \ldots \ldots \ldots \ldots \ldots \ldots$

2.1 Body frame . . . . . . . . . . . . . . . . . . . . . . . 19

2.2 Added-mass factors $[11] \ldots \ldots \ldots \ldots \ldots$

2.3 Fin cross section $\ldots \ldots \ldots \ldots \ldots \ldots \ldots \ldots$

2.4 Body-fin factor $k_{44}[104] \ldots \ldots \ldots \ldots \ldots \ldots$

2.5 Efficiency factor $\eta_{f}[105,106] \ldots \ldots \ldots \ldots \ldots$

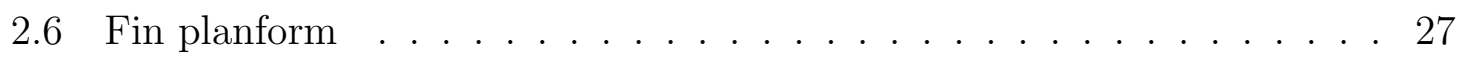

2.7 Efficiency factor $\eta \ldots \ldots \ldots \ldots \ldots$

2.8 Normal force decomposition . . . . . . . . . . . . . . . . . 30

2.9 Normal force on a fin . . . . . . . . . . . . . . . . . . . 31

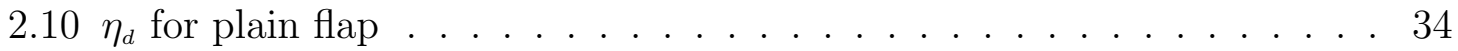

2.113 -D Effectiveness factor for $\tau=0.5 \ldots \ldots \ldots \ldots$

2.12 Profiles of the airships in simulation examples . . . . . . . . . 38

2.13 Steady-state aerodynamics results of the Akron model . . . . . . . . . . 40

2.14 Throttle crank input and airspeed in the acceleration test . . . . . . . 42

2.15 Elevator input and response results at $12.86 \mathrm{~m} / \mathrm{s} \ldots \ldots \ldots$

2.16 Rudder input and response results at $12.86 \mathrm{~m} / \mathrm{s} \ldots \ldots \ldots 44$ 
2.17 Yaw rate in steady turn $($ at $12.86 \mathrm{~m} / \mathrm{s}) \ldots \ldots \ldots \ldots 4$

2.18 Control response in the frequency domain at $12.86 \mathrm{~m} / \mathrm{s} \ldots \ldots . .46$

2.19 Control response in the frequency domain at $20.58 \mathrm{~m} / \mathrm{s} \ldots \ldots . .47$

2.20 Longitudinal modes . . . . . . . . . . . . . . . . . . . . . 49

2.21 Vector diagram of longitudinal modes at $1 \mathrm{~m} / \mathrm{s} \ldots \ldots \ldots$

2.22 Vector diagram of longitudinal modes at $15 \mathrm{~m} / \mathrm{s} \ldots \ldots \ldots$

2.23 Vector diagram of longitudinal modes at $30 \mathrm{~m} / \mathrm{s} \ldots \ldots \ldots$

2.24 Lateral modes . . . . . . . . . . . . . . . . . . . . . . 52

2.25 Vector diagram of lateral modes at $1 \mathrm{~m} / \mathrm{s} \ldots \ldots \ldots \ldots 2$

2.26 Vector diagram of lateral modes at $15 \mathrm{~m} / \mathrm{s} \ldots \ldots \ldots . \ldots 53$

2.27 Vector diagram of lateral modes at $30 \mathrm{~m} / \mathrm{s} \ldots \ldots \ldots$

2.28 An airship in steady turn . . . . . . . . . . . . . . . 55

2.29 Turn rate results (at $12.86 \mathrm{~m} / \mathrm{s}$ ) from 2-DOF and 6-DOF models . . . . 57

3.1 Body frame on the undeformed body $\ldots \ldots \ldots \ldots$

4.1 Local centerline frame . . . . . . . . . . . . . . . 73

4.2 Boundary condition of the Laplace equation $\ldots \ldots \ldots \ldots$

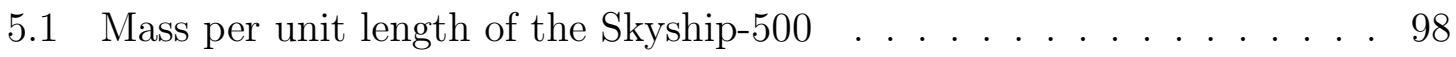

5.2 The first two normal mode shapes . . . . . . . . . . . . . . . . 99

5.3 Elevator and rudder input in Cases I and II . . . . . . . . . . 103

5.4 Time histories of rigid-body velocities, aerodynamic force and moment, Case I, at $30 \mathrm{~m} / \mathrm{s} \ldots \ldots \ldots \ldots \ldots$

5.5 Time histories of generalized coordinates and velocities (Case I) . . 105

5.6 Time histories of rigid-body velocities, aerodynamic force and moment, Case II, at $30 \mathrm{~m} / \mathrm{s} \ldots \ldots \ldots \ldots \ldots \ldots$

5.7 Time histories of generalized coordinates and velocities (Case II) . . . 107

5.8 Desired path in Case III . . . . . . . . . . . . . . . . . 108

5.9 Desired yaw angle and yaw rate in Case III . . . . . . . . . . . 108 
5.10 Time histories of rigid-body velocities, Case III, at $15 \mathrm{~m} / \mathrm{s}$. . . . . . 110

5.11 Time histories of rigid-body velocities, Case III, at $30 \mathrm{~m} / \mathrm{s}$. . . . . . . 111

5.12 Flight path and control input, Case III . . . . . . . . . . . . . . . . . 112

5.13 Control response in the frequency domain at $15 \mathrm{~m} / \mathrm{s} \ldots . . . . . .114$

5.14 Control response in the frequency domain at $30 \mathrm{~m} / \mathrm{s} \ldots \ldots . . . .115$

6.1 Eigenvalues of longitudinal modes from flexible-body dynamics . . . . . 120

6.2 Eigenvalues of lateral modes from flexible-body dynamics . . . . . . . . 121

6.3 Eigenvalues of longitudinal modes from rigid-body dynamics . . . . . . 122

6.4 Eigenvalues of lateral modes from rigid-body dynamics . . . . . . . . . 123

6.5 Cause of the aeroelastic instability . . . . . . . . . . . . 124

6.6 Time histories of vertical speed, pitch angle and aerodynamic pitch moment at $86 \mathrm{~m} / \mathrm{s}$ and $90 \mathrm{~m} / \mathrm{s}$, given a disturbance of $\Delta w_{0}=1 \mathrm{~m} / \mathrm{s} . .126$

6.7 Time histories of vertical speed, pitch angle and aerodynamic pitch moment at $105 \mathrm{~m} / \mathrm{s}$ and $125 \mathrm{~m} / \mathrm{s}$, given a disturbance of $\Delta w_{0}=1 \mathrm{~m} / \mathrm{s} 127$

6.8 Eigenvalues of longitudinal modes from flexible-body dynamics, with material in Eq. (6.1) . . . . . . . . . . . . . . . . . . . 129

6.9 Eigenvalues of lateral modes from flexible-body dynamics, with material in Eq. (6.1) . . . . . . . . . . . . . . . . . 130 


\section{List of Tables}

2.1 Added-mass results . . . . . . . . . . . . . . . . . . . . . . . . . . 39

2.2 Mass, position of C.G. and moments of inertia (about the C.V.) of the Skyship-500 $[17] \ldots \ldots \ldots \ldots \ldots$

4.1 Added-mass matrix entities . . . . . . . . . . . . . . 77

4.2 Velocity distribution corresponding to boundary conditions . . . . . . 83

5.1 Summary of mass matrix and inertial, gravity and aerostatic forces . . 93

5.2 Summary of aerodynamic forces . . . . . . . . . . . . 94

5.3 Control parameters at different speeds _ . . . . . . . . . . . 109 


\section{Nomenclature}

$a, e$

A,B

$b$

$B_{n}^{p}, C_{n}^{p}$

$c, c_{f}$

$C_{D}, C_{L}, C_{M}$

$C_{D C}$

$C_{D H 0}, C_{D F 0}$

$C_{L \alpha}, C_{l \alpha}$

$\mathbf{c}_{\text {total }}$

D

$d_{F}$

E

EI

F

f

$g$

$\hat{\mathrm{g}}$

H

$\mathbf{h}_{i}$

$\mathbf{h}_{i j}^{\prime}$ semi major axis and eccentricity of an ellipsoid of revolution

state matrix and input matrix of the linear model

fin semi span

coefficients in the calculation of velocity potentials in Eq. (4.23)

airfoil chord and flap chord

drag, lift and pitching moment coefficients

cross-flow drag coefficient on the hull

zero-angle axial drag coefficients of the hull and the fins

3-D and 2-D lift curve slopes of the fins

first moment of inertia of the flexible vehicle

maximum cross-sectional diameter of the airship

distance from a point on a fin to a point on the centerline

elastic modulus

bending stiffness

force vector

force per unit length along the centerline

acceleration of gravity

unit vector in the direction of gravity

angular momentum coefficient matrix

angular momentum coefficients

flexible angular momentum coefficients 


$\begin{array}{ll}\mathbf{H}_{u} & \text { flexible angular momentum coefficient matrix } \\ \mathbf{I} & \text { shape function integral } \\ \mathbf{J} & \text { second moment of inertia of the undeformed airship } \\ \mathbf{J}_{t o t a l} & \text { total second moment of inertia of the flexible airship } \\ \mathbf{J}_{u r}, \mathbf{J}_{r u} & \text { rigid/flexible coupling parts of second moment of inertia of the flex- } \\ & \text { ible airship } \\ \mathbf{J}_{u u} & \text { flexible part of second moment of inertia of the flexible airship } \\ \mathbf{K} & \text { elastic stiffness matrix of flexible vehicle } \\ k_{1}, k_{2}, k^{\prime} & \text { added-mass factors of ellipsoids } \\ k_{3 D} & \text { 3-D efficiency factor for the aerodynamics of control surface deflec- } \\ k_{44} & \text { tion } \\ K_{E 1}, K_{E 2} & \text { added-mass factor for the added moment of inertia of the fins } \\ K_{R 1}, K_{R 2} & \text { control gains for elevator deflection } \\ K_{T} & \text { control gains for rudder deflection } \\ L & \text { control gain for thruster } \\ \mathbf{M}_{F, 22}, \bar{m}_{F, 33} & \text { length of the airship } \\ \bar{m}_{F, 44} & \text { moment vector } \\ m_{H i j}, m_{F, i j} & \text { elements of added-mass matrices } \mathbf{M}_{A H} \text { and } \mathbf{M}_{A F} \\ \mathbf{M}_{q q} & \text { mass per unit length } \\ m^{\prime}, I^{\prime} & \text { total mass of the airship } \\ \mathbf{M}_{A H}, \mathbf{M}_{A F} & \text { mass and moment of inertia of the air displaced by the hull } \\ \mathbf{M}_{A, r i g i d} & 6 \times 6 \text { added-mass matrices of the undeformed hull and fins } \\ \mathbf{M}_{A T} & \end{array}$




\begin{tabular}{|c|c|}
\hline $\mathbf{M}_{q s}, \mathbf{M}_{s q}$ & $\begin{array}{l}\text { added-mass matrices associated with the coupling between } \boldsymbol{\Psi}_{q} \text { and } \\
\boldsymbol{\Psi}_{s}\end{array}$ \\
\hline $\mathbf{M}_{\text {rigid }}$ & $6 \times 6$ mass matrix of the undeformed airship \\
\hline $\mathbf{M}_{r q}, \mathbf{M}_{q r}$ & $\begin{array}{l}\text { added-mass matrices associated with the coupling between } \boldsymbol{\Psi}_{r} \text { and } \\
\boldsymbol{\Psi}_{q}\end{array}$ \\
\hline $\mathbf{M}_{r r}$ & added-mass matrix associated with rigid-body motion \\
\hline $\mathbf{M}_{r s}, \mathbf{M}_{s r}$ & $\begin{array}{l}\text { added-mass matrices associated with the coupling between } \boldsymbol{\Psi}_{r} \text { and } \\
\boldsymbol{\Psi}_{s}\end{array}$ \\
\hline $\mathbf{M}_{s s}$ & added-mass matrix associated with $\boldsymbol{\Psi}_{s}$ \\
\hline $\mathbf{M}_{s y s}$ & $(6+2 N) \times(6+2 N)$ total mass matrix of the flexible airship \\
\hline$N$ & $\begin{array}{l}\text { number of shape functions describing the deflection in the } y \text { or } z \\
\text { direction }\end{array}$ \\
\hline $\mathbf{n}$ & unit normal vector of the body surface \\
\hline $\mathbf{M}_{e}$ & elastic generalized mass matrix \\
\hline $\mathbf{P}$ & linear momentum coefficient matrix \\
\hline $\mathbf{p}_{i}$ & linear momentum coefficients \\
\hline$P_{n}^{p}, Q_{n}^{p}$ & Legendre and associated Legendre functions of degree $n$ and order $p$ \\
\hline $\mathrm{Q}$ & generalized elastic force vector \\
\hline q & generalized coordinate vector \\
\hline$q_{0}=1 / 2 \rho V^{2}$ & dynamic pressure \\
\hline$R$ & hull cross-sectional radius \\
\hline $\mathbf{R}$ & rotation matrix from inertial frame to body frame \\
\hline $\mathbf{r}$ & $\begin{array}{l}\text { position vector of a material point from the body-frame origin on the } \\
\text { undeformed airship }\end{array}$ \\
\hline $\mathbf{r}_{G}$ & $\begin{array}{l}\text { position vector of the C.G. from the body-frame origin on the unde- } \\
\text { formed body }\end{array}$ \\
\hline $\mathbf{R}_{p}$ & rotation matrix from body frame to local centerline frame \\
\hline
\end{tabular}




\begin{tabular}{|c|c|}
\hline $\mathbf{r}_{T}$ & $\begin{array}{l}\text { position vector of a thruster from the body-frame origin on the un- } \\
\text { deformed body }\end{array}$ \\
\hline $\mathbf{r}_{V}$ & $\begin{array}{l}\text { position vector of the C.V. from the body-frame origin on the unde- } \\
\text { formed body }\end{array}$ \\
\hline$S$ & hull cross-sectional area \\
\hline$s$ & spanwise position on a fin \\
\hline $\mathbf{S}_{E}$ & generalized elastic force vector \\
\hline$S_{f}$ & flap area \\
\hline$S_{F E}$ & exposed fin area \\
\hline$S_{H}, S_{F}$ & reference areas for $C_{D H 0}$ and $C_{D F 0}$ respectively \\
\hline$T$ & envelope thickness \\
\hline $\mathcal{T}$ & kinetic energy of the flexible airship \\
\hline $\mathcal{T}_{f}$ & kinetic energy of the potential fluid \\
\hline$\overline{\mathbf{U}}, \Delta \mathbf{U}$ & equilibrium control input and disturbance in control input \\
\hline $\bar{u}_{0}$ & equilibrium forward speed \\
\hline $\mathbf{u}$ & elastic displacement \\
\hline$\tilde{u}_{0}, \tilde{w}_{0}$ & desired velocities in a path-following flight \\
\hline$u(c / 4)$ & axial velocity component at the center of $1 / 4$-chord \\
\hline $\mathbf{u}_{G}$ & elastic displacement of the C.G. \\
\hline$u_{\text {unstable }}$ & speed at which aeroelastic instability occurs \\
\hline $\mathbf{u}_{V}$ & elastic displacement of the C.V. \\
\hline$u_{V}, v_{V}, w_{V}$ & velocity components at the position $\varepsilon_{V}$ \\
\hline$V$ & airspeed \\
\hline $\mathbf{v}$ & velocity distribution over the flexible airship \\
\hline $\boldsymbol{v}=\left[\mathbf{v}_{0}^{\mathrm{T}}, \boldsymbol{\omega}^{\mathrm{T}}\right]^{\mathrm{T}}$ & rigid-body generalized velocity vector \\
\hline $\mathbf{v}_{0}=\left[u_{0}, v_{0}, w_{0}\right]^{\mathrm{T}}$ & linear velocity \\
\hline$V_{B}$ & airship volume \\
\hline
\end{tabular}




\begin{tabular}{|c|c|}
\hline $\mathbf{v}_{d}$ & $\begin{array}{l}\text { local velocity on the deformed airship expressed in the local centerline } \\
\text { frame }\end{array}$ \\
\hline $\mathcal{V}$ & elastic potential energy of the flexible vehicle \\
\hline $\mathbf{v}_{f}$ & fluid velocity vector \\
\hline$v_{n}(c / 4)$ & $\begin{array}{l}\text { velocity component perpendicular to the fin surface at the center of } \\
1 / 4 \text {-chord }\end{array}$ \\
\hline$v_{t}(c / 4)$ & $\begin{array}{l}\text { velocity component tangent to the fin surface at the center of } 1 / 4 \text { - } \\
\text { chord }\end{array}$ \\
\hline$w_{d F}$ & downwash due to the fins \\
\hline$\overline{\mathrm{X}}, \Delta \mathrm{X}$ & equilibrium state vector and disturbance in state vector \\
\hline$x_{F s}, x_{F e}$ & $x$-coordinates of the start and end positions of the fins \\
\hline$X, Y, Z$ & coordinates of body-frame origin in the inertial frame \\
\hline$x, y, z$ & coordinates of a point in the body frame \\
\hline$\alpha_{F}, \alpha_{e}$ & geometric and effective angles of attack of the fins \\
\hline$\delta_{E}, \delta_{R}$ & elevator and rudder deflection, respectively \\
\hline$\delta_{T}$ & displacement of throttle crank \\
\hline$\varepsilon$ & longitudinal distance from the nose \\
\hline$\varepsilon_{V}$ & longitudinal position at which the flow ceases to be potential \\
\hline$\eta$ & efficiency factor for the cross-flow drag due to finite length \\
\hline$\eta_{d}$ & correction factor for the flap effectiveness factor \\
\hline$\eta_{f}$ & efficiency factor for the fin added mass due to 3 -D effect \\
\hline$\gamma$ & angle between centerline and velocity vector \\
\hline$\lambda_{1,2}=\sigma \pm \mathrm{j} \omega_{d}$ & eigenvalues \\
\hline $\boldsymbol{\omega}=[p, q, r]^{\mathrm{T}}$ & angular velocity \\
\hline$\Phi_{i}$ & the $i^{\text {th }}$ shape function \\
\hline$\phi, \theta, \psi$ & Euler angles \\
\hline
\end{tabular}




$\begin{array}{ll}\Phi_{F} & \text { angle from the oxz plane to the fin surface plane } \\ \Phi_{i}, \omega_{i} & \text { the } i^{\text {th }} \text { free-free normal mode and natural frequency } \\ \Psi & \text { total velocity potential } \\ \boldsymbol{\Psi}_{q} & \text { velocity potential vector associated with deflection rate } \\ \boldsymbol{\Psi}_{r} & \text { velocity potential vector associated with rigid-body motion } \\ \boldsymbol{\Psi}_{s} & \text { velocity potential vector associated with bending slope } \\ \rho & \text { air density } \\ \boldsymbol{\tau} & \text { forces and moments } \\ \tau & \text { theoretical flap effectiveness factor } \\ \Delta C_{p \alpha} & \partial \Delta C_{p} / \partial \alpha, \text { where } \Delta C_{p} \text { is the pressure coefficient of the airfoil } \\ \zeta, \mu, \vartheta & \text { ellipsoidal coordinates }\end{array}$

Subscripts: (of various terms $\boldsymbol{\tau}, \mathbf{F}, \mathbf{M}, \mathbf{Q}, \mathbf{f}$ and $\mathbf{I}$ )

A added-mass

$A D \quad$ aerodynamics

A, non nonlinear potential-flow aerodynamic terms

$A S \quad$ aerostatics

Axial axial drag

C control

F aerodynamic force acting on the fins

G gravity

$H(F) \quad$ aerodynamic force acting on the hull due to the fins

I inertial

T thrust

$V \quad$ viscous effect on the hull

$\delta \quad$ aerodynamic force due to control surface deflection 


\section{Chapter 1}

\section{Introduction}

\section{$1.1 \quad$ Background}

Airships were the first aircraft that realized human's dream of controlled, powered flight. In 1784, Jean-Baptiste Meusnier proposed a design for an airship of ellipsoid form with a rudder, an elevator and three large airscrews, but he lacked a lightweight, powerful engine. Henri Giffard was the first person to apply steam-engine technology successfully to airships. He flew his airship 17 miles in 1852, with a single propeller driven by a three horsepower engine [1]. The golden age of airships began with the launch of the Luftschiff Zeppelin LZ1 in 1900. The Zeppelin airships became the most famous aircraft of that time and some of these airships were used as bombers during World War I by the German Army. The United States and the Britain built several airships, such as the R-33, R-34 British airships and the American USS Shenandoah (ZR-1), in the 1920s and 1930s, mostly imitating the original Zeppelin design. Initially, airships obtained some degree of great success and an impressive safety record. For example, the German Graf Zeppelin flew over one million miles without a single passenger injury. But the use of airships had declined over time as the development and application of airplanes and a series of airship accidents, including the burning 
of the Hindenburg, the largest aircraft ever built, in 1937. Although the golden age of airships has ended for decades, in the past few years, the development of modern techniques, such as composite materials, optimal design, computational fluid dynamics (CFD), thermal modeling, automatic control, all brought a resurgence of these aircraft. A wide range of applications have recently been proposed for airships, such as advertising, surveillance, environmental monitoring, planetary exploration and stratospheric observation [2-7].

An airship is a lighter-than-air (LTA) aircraft having propulsion and steering systems [8]. Unlike conventional heavier-than-air (HTA) vehicles such as airplanes and helicopters whose lift is aerodynamically generated by moving an airfoil through the air, airships stay aloft using a light lifting gas. This distinguishing feature can provide them long endurance, high payload-to-weight ratio and low fuel consumption.

Airships fall into three main structural categories, namely, rigid airships, non-rigid airships, and semi-rigid airships. Rigid airships have rigid frames containing multiple, non-pressurized gas cells or balloons to provide lift. Non-rigid airships (blimps) use a pressure level in excess of the surrounding air pressure in order to retain their shape. Semi-rigid airships, like blimps, require internal pressure to maintain their shape, but have a rigid keel along the bottom of the envelope to distribute suspension loads into the envelope and allow lower internal pressure. Non-rigid airships are the most common form nowadays. A typical non-rigid airship, shown in Fig. 1.1, has a hull that is filled with light gas, actuated by thrusters and controllable tail fins. The thrusters are usually installed on the gondola; sometimes another one will be installed on the downward vertical fin to provide additional yaw control. At the same time, in order to maintain the aerodynamic shape of the hull, there must be sufficient pressure difference between the surrounding air and the internal lifting gas across the hull envelope. It is necessary to keep this pressure difference despite the influences of varying temperature and altitude; this is achieved through inflating or deflating 


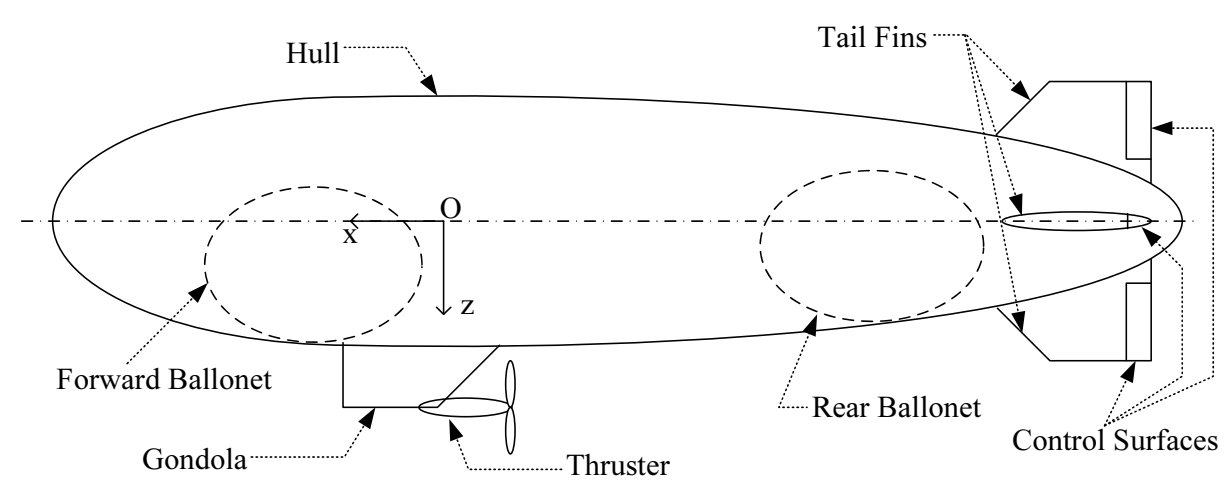

Figure 1.1: Schematic of a non-rigid airship

ballonets, which are air bags contained inside the hull.

The study of modern airships is an interdisciplinary area that involves different theories and technologies. In this thesis, we focus our interests on the dynamics modeling and simulation, which are important tools to analyze their flight behavior and design their control systems. In particular, we focus on a theoretical frame work that can integrate the airships' flight dynamics, structural dynamics, aerostatics and aerodynamics. A review of the research related to airship dynamics and structural analysis is given in the next section.

\section{$1.2 \quad$ Literature Review}

\subsubsection{Dynamics models of airships}

In most dynamics models of aircraft, the vehicles are modeled as a rigid body with three translational and three rotational degrees of freedom (DOF). These dynamics models can be represented by six differential equations, which have been derived in several textbooks for conventional aircraft, such as those by Etkin [9] and Pamadi [10]. However, the large differences between HTA and LTA aircraft imply that models specific to airships must be developed. For example, certain solid-fluid interaction

forces and moments can be neglected for HTA aircraft but become important for 


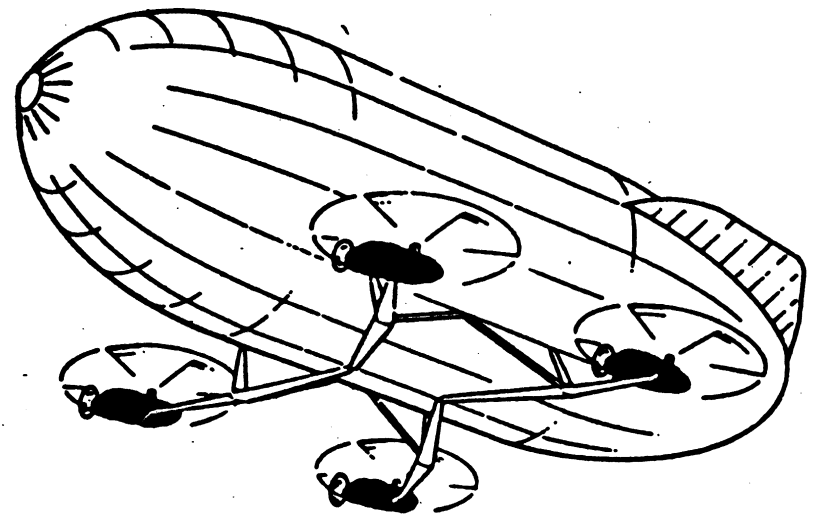

Figure 1.2: Hybrid heavy lift airship in [13]

LTA aircraft, such as buoyancy and those related to the inertia of the surrounding air. The latter ones are usually called the added-mass force and moment, which can be considered as the pressure-induced fluid-structure interaction terms based on a potential flow assumption [11]. A number of airship dynamics models have been presented in the literature in which the airships were also modeled as a rigid-body vehicle.

National Aeronautics and Space Administration (NASA) and Systems Technology, Inc. performed a feasibility study of a hybrid heavy-lift airship, a combination of LTA and rotary wing aircraft. The vehicle consists of a helium-filled envelope mounted on a platform that has a helicopter at each corner, as shown in Fig. 1.2. Tischler et al. [12] derived the nonlinear 6-DOF equations of motion and developed the simulation program HLASIM to evaluate this airship's dynamics characteristics, with a particular focus on the influence of high incidence [13] and atmospheric turbulence [14] on the dynamics model.

The dynamics of other conventional airships were also analyzed. Amann [15] followed the aerodynamics prediction method of Jones and DeLaurier [16] and developed a dynamic simulation program to predict the time responses due to different control inputs for the non-rigid Skyship-500 airship, shown in Fig. 1.3. Jex and Gelhausen [17] 


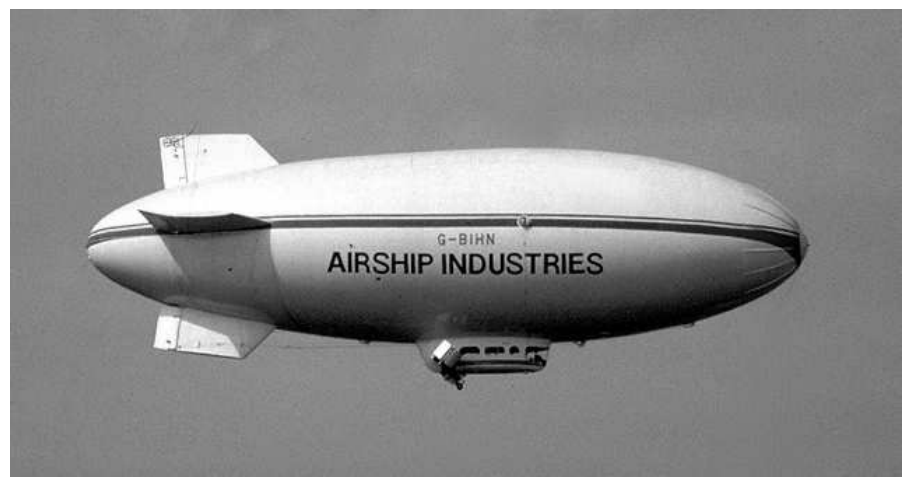

Figure 1.3: The Skyship-500 airship

adapted the HLASIM simulation program to the Skyship-500 to study its flight behavior, and then used a frequency-domain fitting technique to improve the estimates of the aerodynamic derivatives from flight test data.

As with LTA aircraft, an airship's flight characteristics can be strongly affected by atmospheric turbulence. Thomasson [18], Azinheira et al. [19] and Yamasaki and Goto [20] discussed the incorporation of the wind effects (wind speed, acceleration and spatial gradients) into the nonlinear equations of motion of airships, with a special focus on formulating the coupling between the wind effects and the added-mass aerodynamic terms.

Analytical linear dynamics models using aerodynamic derivatives have been applied to study the flight behavior of old rigid airships. For example, Jones and Bell $[21,22]$ used a simplified linear model to investigate the steady turn characteristics for the R-29 and R-101 British rigid airships, with the aerodynamic derivatives measured from wind-tunnel experiments. Linear dynamics models are still used for airships nowadays due to their simplicity and the fact that the models can be readily improved by updating the aerodynamic derivatives obtained from wind-tunnel or flight tests. Cook et al. [23] formulated the linearized equations of motion for a nonrigid airship and investigated its flight stability at various speeds. Schmidt [7] applied a 3-DOF linear model for the control design of a large high-altitude airship. 


\subsubsection{Aerodynamics of airships}

In the above airship models, the computation of aerodynamic force and moment is a key issue. Many theoretical works on the aerodynamics of old airships are based on potential flow theory. Munk [24] applied a slender body assumption to derive the aerodynamic force distribution over an airship hull. The effects of hull's finite length was corrected using the added-mass factors of ellipsoids derived by Lamb [25]. Munk's

results showed that the hull experiences an unstable pitch moment at nonzero angles of attack. Von Karman [26] applied the method of sources and sinks to investigate the pressure on the model of USS Los Angeles (ZR-3) airship. Upson and Klikoff [27] extended Munk's work to formulate the force distribution for an airship hull in curvilinear flight.

However, a prediction based on potential flow cannot represent the real aerodynamics of the hull because of the effects of viscosity, especially at the rear of the body. Allen and Perkins $[28,29]$ added a term related to the cross-flow drag to correct the effects of viscosity for an inclined body of revolution. Hopkins [30] proposed a similar method by assuming that the transverse aerodynamic force on a forward portion of the body could be calculated using the potential flow assumption while the force on the remaining portion was obtained by the cross-flow drag. The position at which the potential flow ceases was then estimated from wind-tunnel test results.

Wind tunnel tests have shown that strong aerodynamic interaction effects exist between the hull and the fins of airships. Several approaches can be found in the literature to compute the aerodynamics of wing-body combination for airplanes and missiles, such as those by Lawrence and Flax [31] and Pitts et al. [32]. Jones and DeLaurier [16] wrote an important paper to address the hull-fin interaction problem for airships and aerostats. They proposed a semi-experimental approach to predict the steady-state aerodynamics, using efficiency factors to account for the interaction between the hull and the fins. These factors were estimated from wind-tunnel test 
results. Then they extended the steady-state model for an airship in general rigidbody motion by discretizing it into slices.

Recently, CFD techniques have been applied to analyze the aerodynamic characteristics of airships. Wong et al. [33] used source panels to model the hull and vortex-lattice panels for the fins. Lutz et al. developed a number of CFD packages to investigate the aerodynamic characteristics of the Lotte airship [34-38], including its steady-state aerodynamic coefficients, added mass and moment of inertia, boundary layer of the hull, and propeller influence. Many of their results were summarized in [39]. In addition, CFD techniques have also been used in airship design. For example, different panel approaches were developed to optimize the hull shape in order to reduce the drag [40-42].

\subsubsection{Wind-tunnel and flight tests}

Wind-tunnel and flight tests have been an important means to establish and evaluate the airship dynamics. First, the aerodynamic characteristics can be obtained in the experiments, and then be used in the dynamics model. On the other hand, the published airship experimental data can be applied to verify the theoretical aerodynamics or dynamics model.

Two important review papers on airship experiments were published. Jones [43] summarized the aerodynamic characteristics of several British airships deduced from experiments in the 1910s and 1920s, and used these results to analyze the flight stability in the horizontal plane. In 1976, Curtiss [44] gave a review of LTA aerodynamic experiments up to that date, especially those by the Aeronautical Research Council $(\mathrm{ARC})$ in Britain and the National Advisory Committee for Aeronautics (NACA) in the United States.

A great amount of wind-tunnel results for old airships are collected in the reports of NACA and ARC, especially for the scale models of the American airships Akron 
(ZRS-4) [45-49], Shenandoah [50], the British airships R-29 [51], R-101 [52], and the German airship L33 [53]. Wind-tunnel tests were also used for the design of modern airships and other LTA vehicle, such as the Lotte airship [54] and the TCOM 250 aerostat [16]. In most of these experiments, researchers measured the steady-state aerodynamic force and moment (drag, lift and pitch moment) at angles of attack, as well as the pressure distribution in some cases such as the Akron [47], R-101 [52] and Lotte [54] models. In addition, the rotational stability derivatives such as yaw rate derivatives and pitch rate derivatives could be measured in a wind tunnel by means of oscillation test [50] or using whirling arms [16].

Flight test results on full-scale airships were also presented in the literature, for example, for the British rigid airships R-26 [55], R-29 [56] and R-33 [57]. In particular, research focused on the turn trials to determine the turn radius at various rudder inputs, because it was difficult to predict the aerodynamic force and moment for airships in curvilinear flight at that time. In addition, the pressure distribution and flight trajectories were recorded for the USS Los Angeles airship in flight tests [58-60]. However, these experimental works were published decades ago and the accuracy of the results was limited by the instrumentation and measurement techniques at that time. More advanced instrumentation has been used in the flight tests of modern airships. In the Patrol Airship Concept Evaluation (PACE) program [61] in 1980s, flight tests were performed for the non-rigid Skyship-500 airship. The time and frequency responses to inputs of elevator, rudder and throttle were measured during the tests. Some of these results were published by Jex and Gelhausen [17,62]. Recently, Kornienko [63] designed an experimental setup to measure the responses of the Lotte airship in maneuvers and applied a system identification approach to determine the aerodynamic derivatives and dynamics characteristics from the flight test data. 


\subsubsection{Structural analysis of airships and related inflated struc- tures}

Real airships experience deformations and their structural flexibility has been an important consideration in the history of airship development. The structural problems in the rigid airship construction were discussed in the review works by Burgess et al. [64] and Ebner [65]. Herrera [66] and Evans [67] applied different approaches to compute the force distribution due to aerostatic, aerodynamic and inertial forces for the R-38 and Shenandoah rigid airships respectively. Their results demonstrated the possibilities of a catastrophic failure due to the structural bending moment for these rigid airships.

To avoid the structural vulnerability, rigid airships are seldom used nowadays and most modern airships are non-rigid airships. The deflection behavior of non-rigid airships was investigated by using a bending beam model [2] and experiments showed that such a model could provide reasonable accuracy $[68,69]$.

With more recent advances in computational capabilities, Finite Element Analysis (FEA) has been used for the preliminary structural analysis of modern airships and other LTA aircraft. Hunt [70,71] and Witherow [72] performed static structural analysis of aerostats to obtain the deformed shape and stress distribution, using FEA packages NASTRAN and LD3DX respectively. In addition, CFD packages have been combined with FEA models to compute the aerodynamics of flexible airships. Amiryants et al. [73] investigated the static elastic deformation and natural modes of a 260-meter semi-rigid airship using FEA packages NASTRAN and ABAQUS and computed the static aerodynamic coefficients with the CFD package ARGON. Their results showed that the first two modes could be described as beam-bending modes and that the structural elasticity could considerably influence the static aerodynamic force and moment. Bessert and Frederich [74] analyzed the effects of deformation on the lift coefficients at angles of attack for the flexible CL-160 non-rigid airship using 
FEA package ABAQUS and CFD solver VSAERO. Their analysis displayed strong effects of the geometric and material nonlinearities of the hull on the aerodynamic derivatives. Omari et al. [75] developed a numerical structure-fluid interaction solver based on a mixed element volume discretization and used this program to obtain the deformation, natural modes and aerodynamic derivatives of a flexible ellipsoid in an inviscid flow at different angles of attack. The computed first and third modes were beam-bending modes and the second mode was a membrane mode. They found that influence of elastic displacement on the aerodynamic coefficients was small.

In general, the majority of the above cited FEA and CFD works on flexible airships focused on the computation of their static deformation, stress, aerodynamic forces and natural modes. However, few studies investigated the influence of flexibility on the dynamics characteristics, and it would be difficult to incorporate the above FEA and CFD models into an airship dynamics simulation program because of the high computational cost. Bennaceur et al. [76] investigated the equations of motion of a flexible airship with a particular focus on the effects of deformation on the inertial force. However, their formulation was limited by assuming the air to be a potential fluid. Furthermore, little discussion was given on the interaction between the flexibility and the aerodynamic forces.

In addition, the structural characteristics of LTA aircraft depend on their envelope materials. Various composite materials have been employed in modern airships, such as polyamides (Nylon) and polyesters (Dacron and Terylene) [2]. Recently, very thin (about $0.048 \mathrm{~mm}$ ) films have been proposed for high-altitude airships, for example, the High Platform II shown in Fig.1.4, the first stratospheric airship (at 23,165 m) in the world [77]. These materials were first developed as part of the NASA's Ultra Long Duration Balloon project [77]. Although they are very light and provide sufficient strength, these films have lower elastic moduli than conventional airship envelope materials such as Nylon and Dacron. Accordingly, particular attention should be 


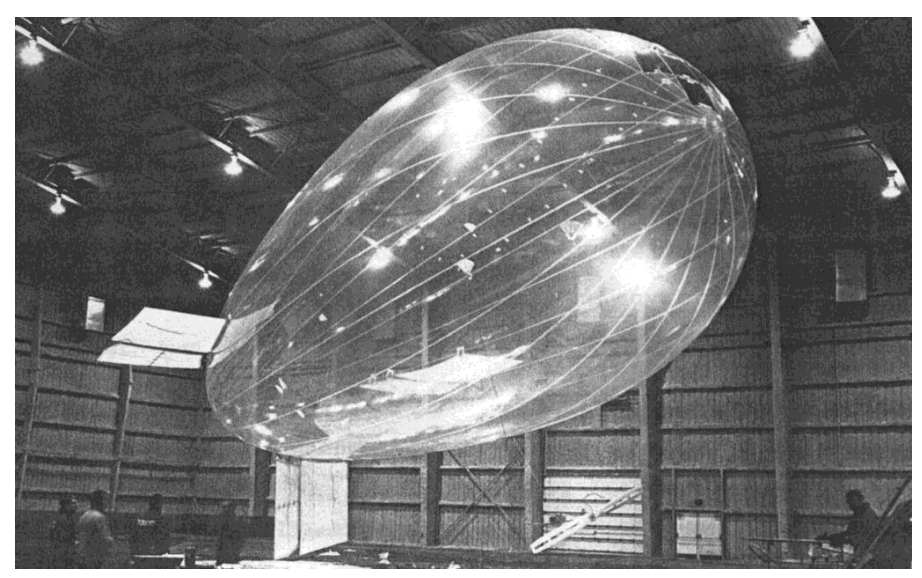

Figure 1.4: The High Platform II airship

focused on the structural-fluid interaction in the airships made of these unconventional composite materials.

\subsubsection{Structural analysis of other inflated structures}

The largest component of a typical modern airship, the hull, is actually an inflated membrane structure. Although research on structural analysis of flexible airships and aerostats is limited, many works can be found on other inflated structures, such as inflated cylinders, inflated toroidal shells and parachutes, based on theoretical models or using commercial FEA packages. Many of these studies have been reviewed in a number of papers [78-81]. Because of the shape of the hull, we focus our discussion here on works on inflated circular cylinders.

A beam model has been applied to model the structural characteristics of inflated cylinders, with the advantage of very easy implementation, although the membrane characteristics are neglected. Main et al. [82] wrote an important paper in 1994 on the load-deflection behavior of cantilevered inflated cylinders. They modeled the cylinders as Euler-Bernoulli beams if the internal pressure was high enough so that no wrinkling occurred, and then their beam assumption was extended to perform the modal analysis [83]. By comparing to experimental results, the beam assumption was 
found to lead to reasonable prediction of the elastic deflection and vibration mode shapes of the first two modes. Similarly, Rybski et al. [84] used the Timoshenko beam model to calculate the natural frequencies of a cantilevered inflatable beam; Suhey et al. [85] used the Euler-Bernoulli beam equation to obtain the deflection of a simplysupported inflated cylinder and their computation results matched the FEA results in ANSYS.

Researchers have also developed more complicated theoretical membrane models to analyze the structural characteristics of inflated structures. For example, Young et al. [86] established a theoretical model for the dynamics of thin-film membranes, and applied their model to obtain the vibration modes of an inflated circular cylinder membrane tube, and then verified their model by comparing the computed and experimental results. In the modal results of their inflated cylinder, the first, second and fifth modes are beam bending modes and the third, fourth and sixth modes are membrane modes.

\subsubsection{Dynamics of flexible HTA aircraft}

Although few prior works exist on the influence of flexibility on aerodynamics and dynamics models of LTA aircraft, there is a large amount of research on the structurefluid interaction of HTA aircraft, especially on the issue of aeroelastic instabilities (divergence and flutter). Aeroelasticity is the study of the interaction between the de-

formation of an elastic structure in the air and the resulting aerodynamic forces [87]. A common model in conventional aeroelasticity is an elastic beam mounted at its root [87-90]. That is, conventional aeroelasticity is mainly concerned with the structural instability of a wing due to the aerodynamic forces. On the other hand, the elasticity also affects the fight dynamics behavior. For example, Etkin [9] included some aeroelastic derivatives in the aerodynamic force computation for the aircraft's dynamics model. Collar suggested that aeroelasticity could be described as forming a 


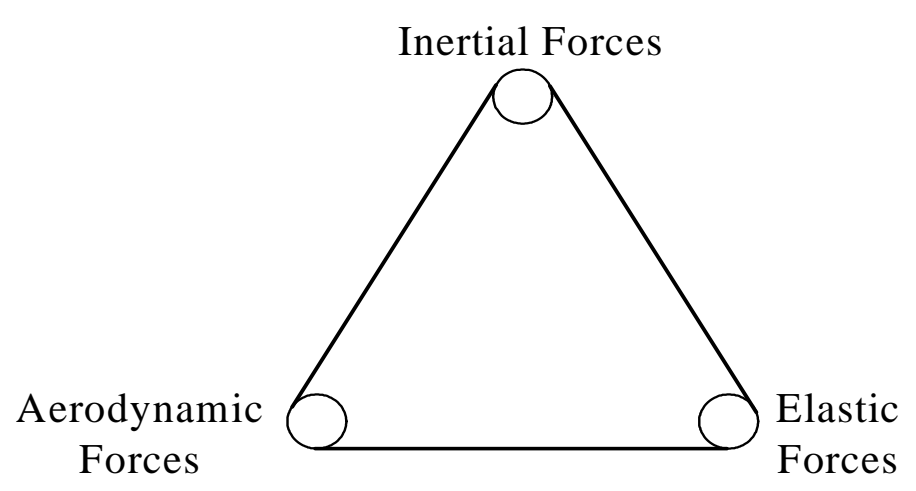

Figure 1.5: Schematic of aeroelasticity [89]

triangle of disciplines, shown in Fig. 1.5 [89], which indicated that the aerodynamics, structural dynamics and flight dynamics could affect each other.

There has been an increasing interest in the modeling of the interaction of flexibility, aerodynamics and flight dynamics of a maneuvering HTA aircraft, especially since powerful computers are available to solve such complicated problems. For example, Bisplinghoff [90], Waszak [91] and Schmidt [92] derived the equations of motion for flexible aircraft in a reference frame moving relative to the undeformed body so that the linear and angular momentum vectors due to elastic deformations vanished. Meirovitch and Tuscu $[93,94]$ presented a procedure to unify the analysis of flight dynamics, structural dynamics, aerodynamics and control system for a flexible airplane, with the body frame established on the undeformed body and the fuselage, wings and tails modeled as several beams. They also provided a review of the works on the flight dynamics and simulation of elastic HTA aircraft. Bolender and Doman [95] followed Meirovitch's method to derive the equations of motion for the longitudinal motion of a hypersonic aircraft. Platus [96] applied a similar approach to analyze the aeroelastic stability of a spinning missile. The coupling between flight dynamics and structural deformation has also been investigated for other HTA aircraft with unconventional configuration. Patil and Hodges [97] derived the dynamics model for a pair of highly flexible flying wings (without fuselage) and then studied the effects of deformation on the phugoid and short-period modes. Cesnik and $\mathrm{Su}$ [98] investigated 
the aeroelasticity of a fully flexible joined-wing vehicle and the effects of flexibility on the time response of its rigid-body motion.

\subsubsection{Vibration of ships}

The coupling between the structural flexibility and fluid-solid interaction forces has also been studied for the other vehicles moving through a heavy fluid. The most well-known instance of this is the hydrodynamic influence on the vibration of ships. Since ships have no wings and move in a heavy fluid, their hydrodynamics modeling is quite different from the aerodynamics of airplanes, and, similar to LTA aircraft, the hydrodynamic terms related to added mass become crucial.

An important paper on ship vibration was written by Lewis [99] in 1929. He modeled a ship as a beam with the shape function chosen as a quadratic function of the longitudinal position, and then investigated the natural frequency of its vertical vibration. He found that that the surrounding water could produce an effect equivalent to a very considerable increase in the mass and decrease in the natural frequency. He first predicted the generalized added mass distribution using strip theory and then developed approximate correction factors for the vibrating ellipsoids to account for the 3-D effects on the generalized added mass distribution.

Macagno and Landweber [100] extended Lewis's work to evaluate the correction factors for other possible vibration mode shapes for an ellipsoid of revolution, based on the analytical solution of the Laplace equation.

Armand and Orsero [101] and Vorus and Hylarides [102] developed different numerical approaches to evaluate the hydrodynamic generalized added-mass matrix for ship vibrations by solving the Laplace equation numerically. In particular, Armand and Orsero [101] showed that, in the dynamics equations of motion for a flexible ship, the generalized added-mass matrix should be included. 


\subsection{Thesis Objectives and Organization}

The overall purpose of this thesis is to improve the understanding of the dynamic behavior of airships, with a particular emphasis on flexible airships. Referring to Collar's diagram in Fig. 1.5, the existing airship dynamics models focus on the interaction of inertial and aerodynamic forces, while the effects of structural flexibility are seldom discussed. The dynamics modeling techniques of flexible airships have lagged behind those for HTA aircraft and this research aims to fill this gap.

For this purpose, a dynamics model is first proposed for airships based on a rigidbody assumption in Chapter 2, with a particular focus on a comprehensive approach to compute the aerodynamic forces and moments and on the model validation. The aerodynamic forces are categorized into various terms based on different physical effects: the added-mass force, the viscous effect on the hull, the force on the fins, the force on the hull due to the fins, and the axial drag. Computational methods are provided for each aerodynamic term and incorporated into the nonlinear dynamics model. The simulation results are then verified by comparing to existing wind-tunnel and flight test data.

Chapters 3 to 6 are devoted to the theory of modeling the dynamics of flexible airships and the corresponding simulation results.

Chapter 3 gives the kinematic description and equations of motion for a flexible airship. The airship is modeled as a free-free Euler-Bernoulli beam and the bending deformations are written using the natural vibration modes as shape functions and the generalized coordinates. Once the velocity distribution of the elastic airship is obtained, Lagrange's equations are applied to derive the dynamics model that describe the linear elastic deformation and the rigid-body motion of the airship. Then the inertial, gravity, aerostatic and thrust forces are incorporated, including the effects of structural flexibility. As a result, the dynamics model is represented by a single set of ordinary differential equations that unifies the airship's flight dynamics, structural 
dynamics, aerostatics and aerodynamics.

To complete the dynamics model, the aerodynamics model verified in Chapter 2 is extended for the flexible airships in Chapter 4. In order to capture the coupling between aerodynamic forces and structural elasticity, the local velocity distribution on the deformed aircraft is used in the computation of aerodynamic forces and moments.

The dynamics model is implemented for an elastic airship in Chapter 5. The simulation results of shape functions, shape function integrals, time and frequency responses to different control surface deflection inputs are presented.

The possibility of aeroelastic instability is investigated for an airship in Chapter 6 . The aeroelastic instability is associated with the interaction of the rigid-body motion, the elastic deformation and the aerodynamic forces.

In Chapter 7, the conclusions drawn in the previous chapters are revisited and recommendations for future research are given.

\subsection{Note on Notation}

As much as possible, the author tried to follow conventional notations used in the different fields pertaining to this thesis. This, however, resulted in a number of notational conflicts - the same symbol used to denote different physical variables. Where deemed not too detrimental to the presentation, some of the original conventions were changed, but several conflicts still remain. For example, $q$ usually denotes a generalized coordinate in the context of structural analysis, a pitch rate in flight dynamics and dynamic pressure in aerodynamics. 


\section{Chapter 2}

\section{Dynamics Model Based on a Rigid-Body Assumption}

Before addressing the dynamics of flexible airships, it is useful to first study the modeling based on a rigid-body assumption. As mentioned in Section 1.2.1, a number of such nonlinear $[12,15,17]$ and linear [21-23] airship models have been presented in the literature. The analytical linear dynamics models with aerodynamic stability derivatives have the advantage that they can be readily improved by updating the aerodynamic derivatives obtained from wind-tunnel or flight tests. On the other hand, in case such experimental tests are not possible, an effective aerodynamic prediction approach can be very useful. An important limitation of the airship dynamics models presented by other authors is that relatively little detail is given on the aerodynamic force prediction. Furthermore, most of these models have not been validated against the actual flight test results.

In this chapter, a modeling approach is proposed to assemble the nonlinear equations of motion based on a rigid-body assumption for the airships, with a particular focus on a comprehensive formulation of the interaction forces between the aircraft and the air, and on the model validation. For this purpose, the derivation begins 
from the equations of motion of a rigid-body vehicle moving in vacuum in Section 2.1. Then the relevant solid-fluid interaction forces, both aerostatic and aerodynamic, are incorporated into the equations in Section 2.2 and 2.3. A linearization procedure is discussed in Section 2.4 to obtain the linear equations of motion. In Section 2.5, the nonlinear dynamics model is used to simulate the Skyship-500 airship and the simulation results are compared to flight test results available in the open literature. Furthermore, the linearized model is applied to investigate the stability of the Skyship500. Finally, a simplified 2-DOF dynamics model is presented to predict the flight behavior for an airship in a steady turn.

With the trust gained from validation, the dynamics model and simulation program in this chapter can be applied to other airships, and the computational method for aerodynamics can be further extended to flexible airships.

\subsection{Equations of Motion for Airship in Vacuum}

The modeling begins from the simplest case of a rigid-body airship moving in vacuum. A local body-fixed frame $\{0 x y z\}$ and an inertial frame $\{O X Y Z\}$ are established respectively, as shown in Fig. 2.1. The airship's position is described by coordinates of the origin of body-fixed frame in the inertial frame, $(X, Y, Z)$; its orientation is represented by Euler (roll $\phi$, pitch $\theta$ and yaw $\psi$ ) angles. These Euler angles are defined by the following sequence of rotations to obtain the axes $\{x y z\}$ from axes $\{X Y Z\}$ : first rotate the axes $\{X Y Z\}$ an angle $\psi$ about the $Z$ direction to axes

$\left\{x_{1} y_{1} z_{1}\right\}$, then rotate the $\left\{x_{1} y_{1} z_{1}\right\}$ an angle $\theta$ about $y_{1}$ direction to axes $\left\{x_{2} y_{2} z_{2}\right\}$ and finally rotate the $\left\{x_{2} y_{2} z_{2}\right\}$ an angle $\phi$ about $x_{2}$ to the axes $\{x y z\}$. The resulting 


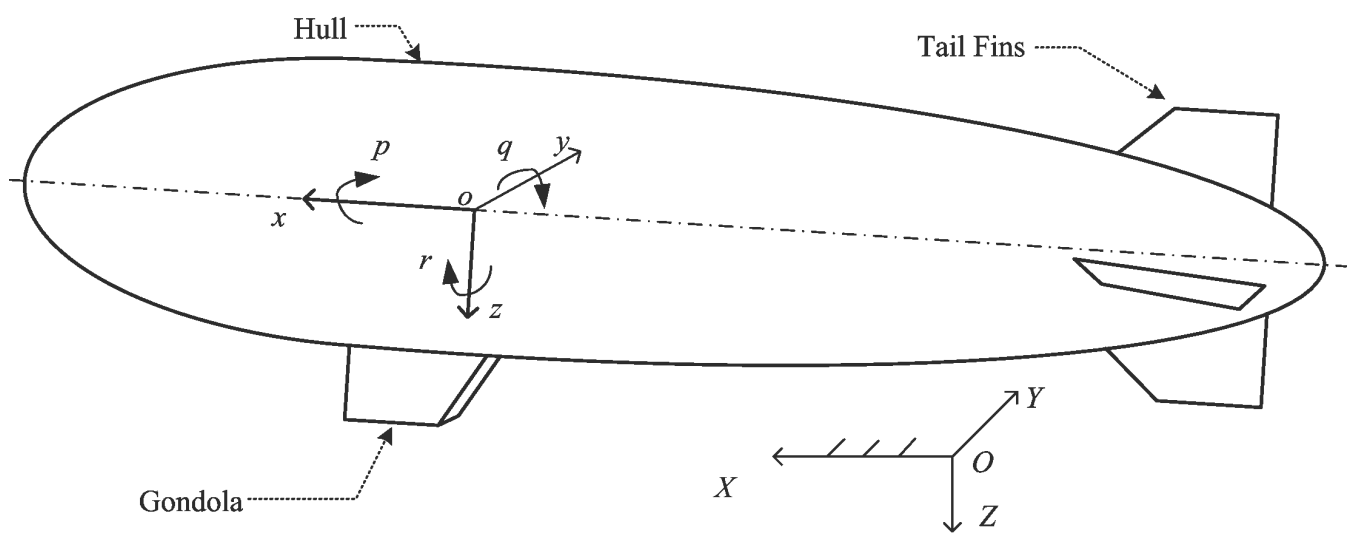

Figure 2.1: Body frame

rotation matrix from the inertial frame to the body frame is [9]

$$
\mathbf{R}=\left[\begin{array}{ccc}
\cos \psi \cos \theta & \sin \psi \cos \theta & -\sin \theta \\
\cos \psi \sin \theta \sin \phi-\sin \psi \cos \phi & \sin \psi \sin \theta \sin \phi+\cos \psi \cos \phi & \cos \theta \sin \phi \\
\cos \psi \sin \theta \cos \phi+\sin \psi \sin \phi & \sin \psi \sin \theta \cos \phi-\cos \psi \sin \phi & \cos \theta \cos \phi
\end{array}\right]
$$

For convenience, the equations of motion for a 6-DOF vehicle are usually derived in the body frame. Usually, the body-fixed frame is located at the center of gravity (C.G.) for an airplane so that the centrifugal terms are eliminated and the translation and rotation are dynamically decoupled, while in this work, we establish the body frame at the center of volume (C.V.) for an airship, with the $x$ axis along the centerline and pointing to the nose, the $z$ axis positively downward, and the positive $y$ axis determined by the right hand rule. The selection of the airship's body-fixed frame location results from three facts: first, the actual C.G. location shifts during the flight due to the motion and inflation/deflation of the ballonets; second, the use of a body frame at the C.V. can simplify the added-mass matrix, as discussed later in Section 2.3.1.3; third, the translation and rotation remain coupled for an elastic airship even if the body frame is established at the C.G., as discussed later in Section 3.3.

For a body moving in vacuum, the equations of motion have been derived in standard textbooks $[9,11]$ using the Newton-Euler approach, and can be summarized 
in matrix form as

$$
\mathbf{M}_{\text {rigid }} \dot{\boldsymbol{v}}=\boldsymbol{\tau}_{I}+\boldsymbol{\tau}_{G}+\boldsymbol{\tau}_{C}
$$

where $\boldsymbol{v}=\left[\mathbf{v}_{0}^{\mathrm{T}}, \boldsymbol{\omega}^{\mathrm{T}}\right]^{\mathrm{T}}$ and $\mathbf{v}_{0}=\left[u_{0}, v_{0}, w_{0}\right]^{\mathrm{T}}, \boldsymbol{\omega}=[p, q, r]^{\mathrm{T}}$ denote the linear and angular velocity vectors expressed in the body frame respectively; $\mathbf{M}_{\text {rigid }}$ is the $6 \times 6$ mass matrix of the rigid body and can be written as

$$
\mathbf{M}_{\text {rigid }}=\left[\begin{array}{cc}
m \mathbf{I}_{3 \times 3} & -m \mathbf{r}_{G}^{\times} \\
m \mathbf{r}_{G}^{\times} & \mathbf{J}
\end{array}\right]
$$

where $m$ is the total mass, including the hull, gas, gondola, fins, ballonets, etc; J is the second moment of inertia and $\mathbf{r}_{G}$ is the position vector of the C.G. from the origin $o$. Note that $\mathbf{J}$ and $\mathbf{r}_{G}$ are both expressed in the body frame. The superscript $\times$ denotes the skew-symmetric matrix form of a vector (corresponding to a cross-product operation).

The right hand side of Eq. (2.2) consists of the external forces and moments. The subscripts $I, G$ and $C$ denote the terms associated with inertia, gravity and control respectively. The inertial and gravity forces and moments are calculated as

$$
\boldsymbol{\tau}_{I}=\left[\begin{array}{c}
-m \boldsymbol{\omega}^{\times} \mathbf{v}_{0}+m \boldsymbol{\omega}^{\times} \mathbf{r}_{G}^{\times} \boldsymbol{\omega} \\
-m \mathbf{r}_{G}^{\times} \boldsymbol{\omega}^{\times} \mathbf{v}_{0}-\boldsymbol{\omega}^{\times} \mathbf{J} \boldsymbol{\omega}
\end{array}\right], \quad \boldsymbol{\tau}_{G}=\left[\begin{array}{c}
\mathbf{F}_{G} \\
\mathbf{r}_{G}^{\times} \mathbf{F}_{G}
\end{array}\right]
$$

where the gravitational force is

$$
\mathbf{F}_{G}=m g \hat{\mathbf{g}}
$$

in which $g$ is the acceleration of gravity. The unit vector $\hat{g}$ in the body-fixed frame is in the direction of gravity, $\hat{\mathbf{g}}=[-\sin \theta, \cos \theta \sin \phi, \cos \theta \cos \phi]^{\mathrm{T}}$.

The control force and moment in $\boldsymbol{\tau}_{C}$ are due to the thrusters, the deflection of control surfaces, and the inflation or deflation of ballonets, and are generated by the automatic control system or the pilot's commands. Some of these forces are 
generated aerodynamically, such as those from the control surface deflection, and will be investigated in Section 2.3. The thrust force depends on the airship's engine and propeller and will be discussed in Section 2.5.2.1 for the simulated Skyship-500 airship.

\subsection{Interaction Forces and Moments Between Air- ship and Air: Aerostatics}

We can now start to incorporate the interaction forces and moments between the vehicle and the air, which include two components: aerostatics and aerodynamics. The former is due to the static air pressure and is independent of the motion of the vehicle while the latter is related to its motion.

The aerostatic force and moment expressed in the body frame are

$$
\boldsymbol{\tau}_{A S}=\left[\begin{array}{c}
\mathbf{F}_{A S} \\
\mathbf{r}_{V}^{\times} \mathbf{F}_{A S}
\end{array}\right]
$$

where the aerostatic force is

$$
\mathbf{F}_{A S}=-\rho g V_{B} \hat{\mathbf{g}}
$$

in which $V_{B}$ is the volume of the airship, while $\rho$ is the air density. Since the body frame is established at the C.V., the position vector of the C.V. relative to the body-fixed frame origin $\mathbf{r}_{V}=\mathbf{0}$ and the aerostatic moment is zero. To incorporate the aerostatics into the equations of motion, $\boldsymbol{\tau}_{A S}$ is added to the right hand side of Eq. (2.2). 


\subsection{Interaction Forces and Moments Between Air- ship and Air: Aerodynamics}

Unlike an airplane, the largest component of an airship is the hull rather than the planar lifting surfaces (e.g. wing, fins). Moreover, the hull-fin interaction plays an important role on the aerodynamics of the airship. Most significantly, the aerodynamic effects related to the inertia of the surrounding air must be considered for an airship due to the light lifting gas. The large differences between an airship and a conventional airplane imply that aerodynamic computation methods specific to airships must be developed. The aerodynamic characteristics of airships were initially investigated during the golden age of airships. For example, Munk [24] first used potential flow theory to compute the aerodynamic force on airship hull, and the viscous effects were then incorporated to improve the estimation accuracy [28-30]. However, the hullfin interaction was still difficult to predict at that time, and therefore wind-tunnel tests became the most important means to obtain the aerodynamic coefficients of an airship. Prediction methods for the hull-fin interaction were later investigated in the fields of missiles and airplanes, such as by Lawrence and Flax [31] and Pitts et al. [32]. However, they were not applied to airships because the development of airships had declined by that time. Therefore, existing analytical aerodynamic models of an airship are incomplete.

In this thesis, a comprehensive aerodynamic computational approach is proposed. First the aerodynamic forces are categorized into various terms based on different physical effects. Then, computational methods for each of these terms are unified and applied to airships. These methods represent the state-of-the-art techniques available in the literature on airship aerodynamics. 


\subsubsection{Added-mass force and moment}

\subsubsection{Force and moment computation from the added-mass matrix}

The added-mass force and moment can be considered as the pressure-induced fluidstructure interaction terms based on the potential flow assumption with the boundary condition that the fluid attaches on the body surface [11]. For a rigid body completely submerged in an unbounded fluid, the added-mass terms can be derived via an energy approach in terms of Kirchhoff's equations $[11,25]$ or alternatively by using Bernoulli's equation to find the pressure distribution over the body [103]. Here we will not present detailed derivations but only review the results. Thus, we can define a $6 \times 6$ symmetric added-mass matrix for a rigid body as

$$
\mathbf{M}_{A, \text { rigid }}=\left[\begin{array}{ll}
\mathbf{M}_{11} & \mathbf{M}_{12} \\
\mathbf{M}_{21} & \mathbf{M}_{22}
\end{array}\right]
$$

The matrices $\mathbf{M}_{11}, \mathbf{M}_{12}, \mathbf{M}_{21}, \mathbf{M}_{22}$ are $3 \times 3$ submatrices, whose elements can be estimated using the methods to be discussed in Section 2.3.1.2. The corresponding added-mass force and moment are then written in vector form as

$$
\boldsymbol{\tau}_{A}=-\left[\begin{array}{ll}
\mathbf{M}_{11} & \mathbf{M}_{12} \\
\mathbf{M}_{21} & \mathbf{M}_{22}
\end{array}\right]\left[\begin{array}{c}
\dot{\mathbf{v}}_{0} \\
\dot{\boldsymbol{\omega}}
\end{array}\right]-\left[\begin{array}{c}
\boldsymbol{\omega}^{\times}\left(\mathbf{M}_{11} \mathbf{v}_{0}+\mathbf{M}_{12} \boldsymbol{\omega}\right) \\
\mathbf{v}_{0}^{\times}\left(\mathbf{M}_{11} \mathbf{v}_{0}+\mathbf{M}_{12} \boldsymbol{\omega}\right)+\boldsymbol{\omega}^{\times}\left(\mathbf{M}_{21} \mathbf{v}_{0}+\mathbf{M}_{22} \boldsymbol{\omega}\right)
\end{array}\right]
$$

Thus, $\boldsymbol{\tau}_{A}$ includes two terms: one related to the time rates of change of the linear and angular velocities of the airship, the other related to the coupling of the linear and angular velocities. Note that for an airship in steady translation, only the moment term $-\mathbf{v}_{0}^{\times}\left(\mathbf{M}_{11} \mathbf{v}_{0}\right)$ appears in Eq. (2.9), which is known as Munk moment [24]; these moments tends to destabilize the pitch and yaw rotations. To incorporate the addedmass force and moment into the dynamics model, the first term in Eq. (2.9) is written on the left hand side of Eq. (2.2) so that the mass matrix $\mathbf{M}_{\text {rigid }}$ is replaced by 


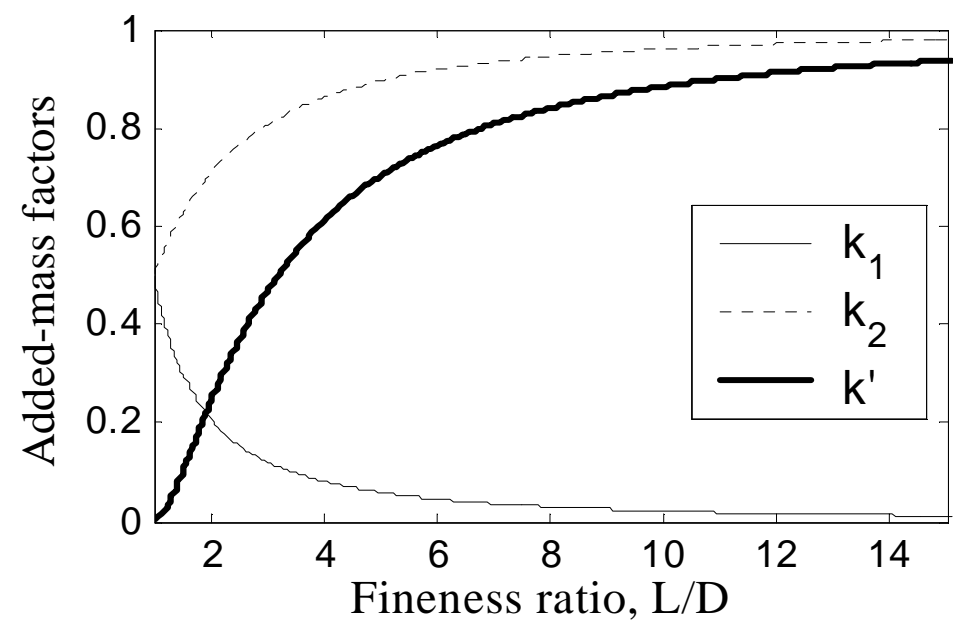

Figure 2.2: Added-mass factors [11]

$\mathbf{M}_{\text {rigid }}+\mathbf{M}_{A, r i g i d}$; while the second term is added to the right hand side of Eq. (2.2).

\subsubsection{Estimation of the added-mass matrix}

The added-mass matrix of a rigid-body airship includes the contributions of both the hull and the fins, i.e.,

$$
\mathbf{M}_{A, \text { rigid }}=\mathbf{M}_{A H}+\mathbf{M}_{A F}
$$

In practice, a simple approach to obtain the added mass and moment of inertia of the hull, $\mathbf{M}_{A H}$, is to approximate the hull as an ellipsoid of revolution. With a body frame defined as discussed in Section 2.1, all the off-diagonal terms in the added-mass matrix of the hull are zero and the diagonal terms are given by

$$
m_{H 11}=k_{1} m^{\prime}, \quad m_{H 22}=m_{H 33}=k_{2} m^{\prime}, \quad m_{H 44}=0, \quad m_{H 55}=m_{H 66}=k^{\prime} I^{\prime}
$$

where $m^{\prime}$ is the mass of air displaced by the hull and $I^{\prime}$ is the moment of inertia of the displaced air. The added-mass factors $k_{1}, k_{2}$ and $k^{\prime}$ [11] are plotted in Fig. 2.2 as functions of the fineness ratio $L / D$, where $L$ is the length of the hull and $D$ is its maximum diameter. 


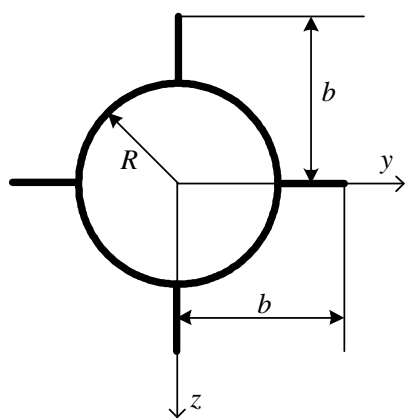

Figure 2.3: Fin cross section

The added mass and moment of inertia of the fins can be computed by integrating the 2-D added mass of the cross section over the fin region. For example, for the cross section with cruciform fins shown in Fig. 2.3, the 2-D transverse added mass in the $y$ and $z$ directions, and the added moment of inertia about the $x$ axis can be computed using potential flow theory as discussed by Nielsen [104]. The contribution of the fins to these 2-D added mass terms can be written as

$$
\bar{m}_{F, 22}=\bar{m}_{F, 33}=\rho \pi\left(b-\frac{R^{2}}{b}\right)^{2}, \quad \bar{m}_{F, 44}=\frac{2}{\pi} k_{44} \rho b^{4}
$$

where $R$ is the hull's cross-sectional radius and $b$ is the fin semi span. The factor $k_{44}$ is plotted in Fig. 2.4 as a function of $R / b$. The 2-D added mass of cross sections with other fin arrangements was also investigated by Nielsen [104].

The non-zero elements in the added-mass matrix of the fins, $\mathbf{M}_{A F}$, are obtained from the following integrals of the 2-D added mass terms in Eq. (2.12)

$$
\begin{aligned}
m_{F 22}=m_{F 33} & =\eta_{f} \int_{x_{F s}}^{x_{F e}} \bar{m}_{F, 22} d x, \quad m_{F 35}=-\eta_{f} \int_{x_{F s}}^{x_{F e}} \bar{m}_{F, 22} x d x, \quad m_{F 26}=-m_{F 35} \\
m_{F 44} & =\eta_{f} \int_{x_{F s}}^{x_{F e}} \bar{m}_{F, 44} d x, \quad m_{F 55}=m_{F 66}=\eta_{f} \int_{x_{F s}}^{x_{F e}} \bar{m}_{F, 22} x^{2} d x
\end{aligned}
$$




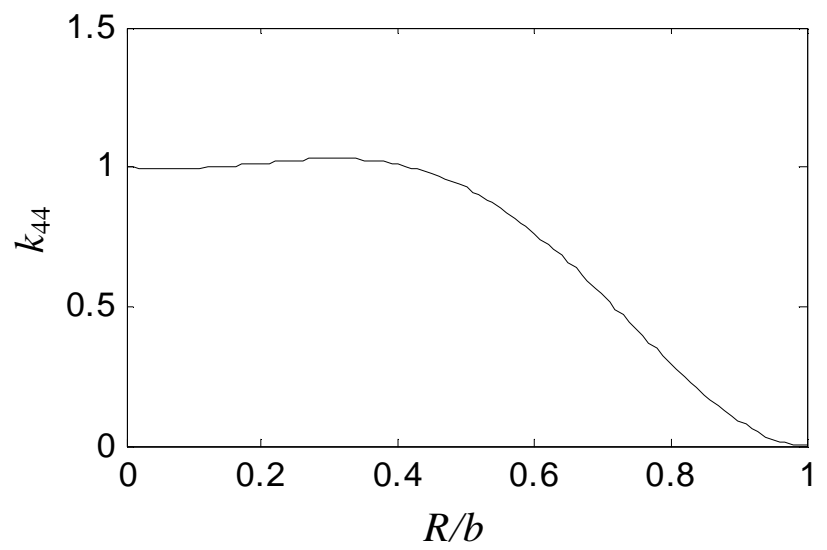

Figure 2.4: Body-fin factor $k_{44}[104]$

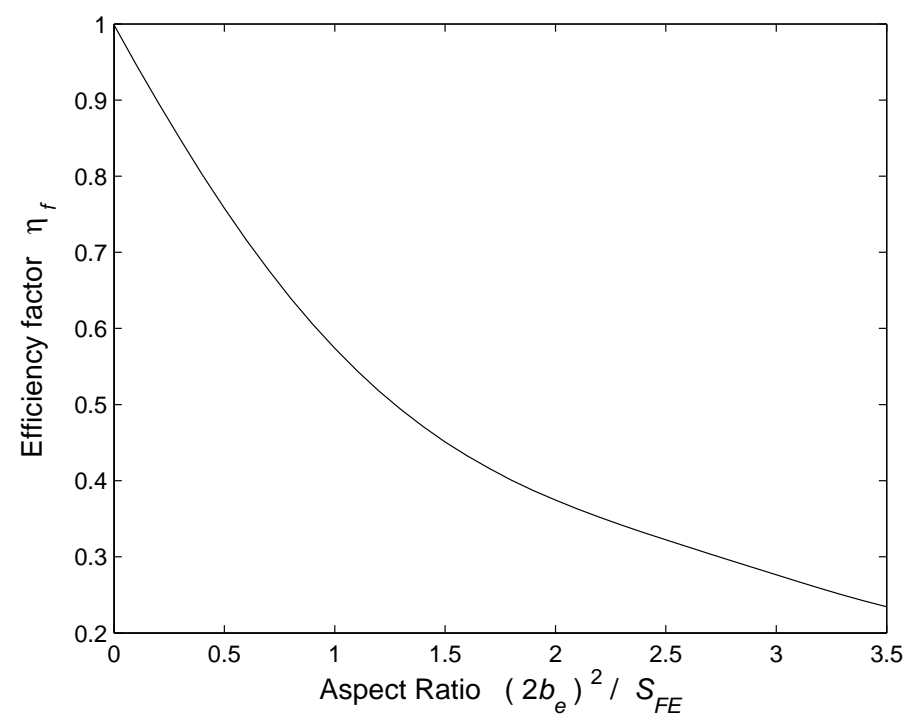

Figure 2.5: Efficiency factor $\eta_{f}[105,106]$

where $x_{F s}$ and $x_{F e}$ are the $x$ coordinates of the start and end positions of the fins. An efficiency factor $\eta_{f}$ (plotted in Fig. 2.5) is included to account for 3-D effects; it is calculated from potential flow theory for the added mass of a thin plate $[105,106]$ and determined based on the aspect ratio $\left(2 b_{e}\right)^{2} / S_{F E}$, in which $S_{F E}$ is the exposed fin area, as shown in Fig. 2.6, and $b_{e}$ is the corresponding semi fin span. 


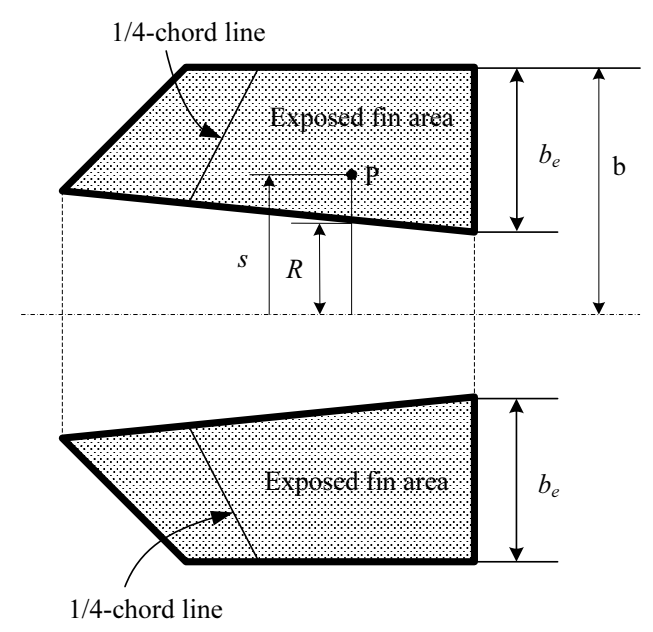

Figure 2.6: Fin planform

\subsubsection{Added-mass matrix transformation}

In the estimation approach for the hull, the body frame is located at the C.V. and oriented along the hull's principal axes. In order to obtain alternative formulations of the equations of motion, with a body frame located at a different position or with different orientation, it is relevant to be able to transform the added-mass matrix between different body frames.

Consider two body frames $\{o x y z\}$ and $\left\{o^{\prime} x^{\prime} y^{\prime} z^{\prime}\right\}$, with the position vector of $o^{\prime}$ in the frame $\{o x y z\}$ being $\mathbf{r}_{\mathrm{o}^{\prime}}$ while the rotation matrix from frame $\left\{o^{\prime} x^{\prime} y^{\prime} z^{\prime}\right\}$ to $\{o x y z\}$ is denoted as $\mathbf{R}_{o^{\prime} o}$. If the added-mass matrix written in the frame $\{$ oxyz $\}$ is $\mathbf{M}_{A, \text { rigid }}$, then it can be written in $\left\{o^{\prime} x^{\prime} y^{\prime} z^{\prime}\right\}$ using the following equation

$$
\mathbf{M}_{A, \text { rigid }}^{\prime}=\mathbf{U}_{o^{\prime} o}^{\mathrm{T}} \mathbf{M}_{A, \text { rigid }} \mathbf{U}_{o^{\prime} o} \quad \text { where } \quad \mathbf{U}_{o^{\prime} o}=\left[\begin{array}{cc}
\mathbf{R}_{o^{\prime} o} & 0 \\
0 & \mathbf{R}_{o^{\prime} o}
\end{array}\right]\left[\begin{array}{cc}
\mathbf{I}_{3 \times 3} & \left(\mathbf{R}_{o^{\prime} o}^{\mathrm{T}} \mathbf{r}_{\mathbf{o}^{\prime}}\right)^{\times} \\
0 & \mathbf{I}_{3 \times 3}
\end{array}\right]
$$

Equation (2.14) can be obtained from the invariance of the kinetic energy of the fluid. If the body frame is established the C.G., the corresponding added-mass matrix can be obtained using Eq. (2.14), and this matrix will have many more non-zero elements than that computed based on a body frame at the C.V. As a result, the body frame 
of a LTA vehicle is usually defined at the C.V.

\subsubsection{Viscous effect on the hull, normal to the centerline}

Wind tunnel tests on the aerodynamics of bodies of revolution at non-zero angles of attack have shown that a prediction based on a potential flow assumption can cause considerable error because of the effects of viscosity, especially at the rear of the body. A semi-empirical estimation approach is given in [30] and [107] for the aerodynamics

of bodies of revolution; in this approach, the force normal to the centerline due to viscous effects can be computed as

$$
F_{V N}=-q_{0} \sin 2 \gamma \cdot\left(k_{2}-k_{1}\right) \int_{\varepsilon_{V}}^{L} \frac{d S}{d \varepsilon} d \varepsilon+q_{0} \eta C_{D C} \sin ^{2} \gamma \cdot \int_{\varepsilon_{V}}^{L} 2 R d \varepsilon
$$

where $C_{D C}$ is the cross-flow drag coefficient of an infinite-length circular cylinder, $\eta$ is an efficiency factor accounting for the finite length of the body and determined from its fineness ratio (shown in Fig. 2.7), $R$ and $S$ are the local cross-sectional radius and area, $\varepsilon$ denotes the longitudinal position from the nose and $\varepsilon_{V}$, the location at which the flow ceases to be potential, is empirically determined as [30]:

$$
\varepsilon_{V}=0.378 L+0.527 \varepsilon_{1}
$$

where $\varepsilon_{1}$ denotes the position at which $d S / d \varepsilon$ has a maximum negative value. The dynamic pressure $q_{0}$ and the angle between centerline and velocity vector $\gamma$ (shown in Fig. 2.8) is computed from the local velocity $\left(u_{V}, v_{V}, w_{V}\right)$ at the position $\varepsilon_{V}$, i.e.,

$$
\gamma=\tan ^{-1}\left(\frac{\sqrt{v_{V}^{2}+w_{V}^{2}}}{u_{V}}\right)
$$

The first term in Eq. (2.15) effectively removes the inviscid flow contribution downstream of $\varepsilon_{V}$ while the second term replaces it with a viscous flow contribution. The 


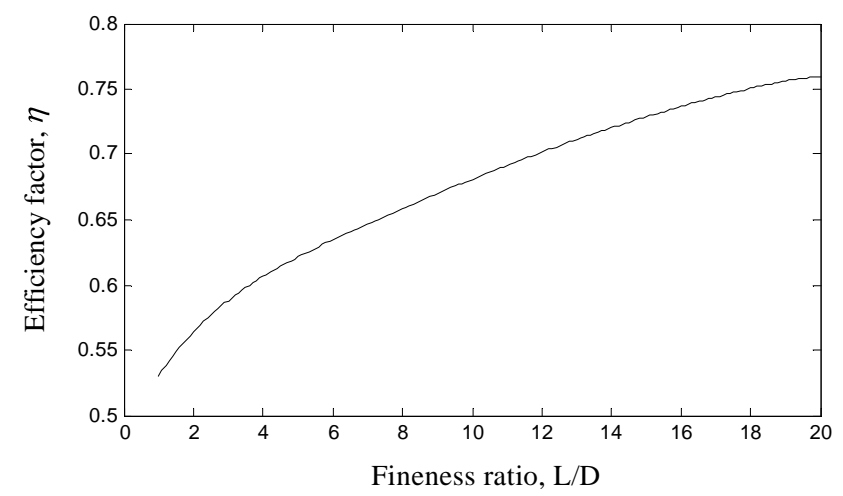

Figure 2.7: Efficiency factor $\eta$

force vector $\mathbf{F}_{V}$ can be written as

$$
\mathbf{F}_{V}=F_{V N}\left[0, \frac{-v_{V}}{\sqrt{v_{V}^{2}+w_{V}^{2}}}, \frac{-w_{V}}{\sqrt{v_{V}^{2}+w_{V}^{2}}}\right]^{\mathrm{T}}
$$

The corresponding moment about the origin of the body frame is computed as

$$
M_{V N}=-q_{0} \sin 2 \gamma \cdot\left(k_{2}-k_{1}\right) \int_{\varepsilon_{V}}^{L} \frac{d S}{d \varepsilon}\left(\varepsilon_{m}-\varepsilon\right) d \varepsilon+q_{0} \eta C_{D C} \sin ^{2} \gamma \cdot \int_{\varepsilon_{V}}^{L} 2 R\left(\varepsilon_{m}-\varepsilon\right) d \varepsilon
$$

where $\varepsilon_{m}$ is the position of the origin of body frame from the nose. The moment vector is then obtained from $M_{V N}$ as

$$
\mathbf{M}_{V}=M_{V N}\left[0, \frac{w_{V}}{\sqrt{v_{V}^{2}+w_{V}^{2}}}, \frac{-v_{V}}{\sqrt{v_{V}^{2}+w_{V}^{2}}}\right]^{\mathrm{T}}
$$

Equations (2.17) and (2.19) are applied to compute the normal forces due to viscosity on the hull and the corresponding moments. These forces and moments are then added to the right hand side of Eq. (2.2).

\subsubsection{Force acting on the fins, normal to the centerline}

We now turn our attention to the force produced by the fins, normal to the airship centerline. This is obtained by estimating the force distribution and integrating it 


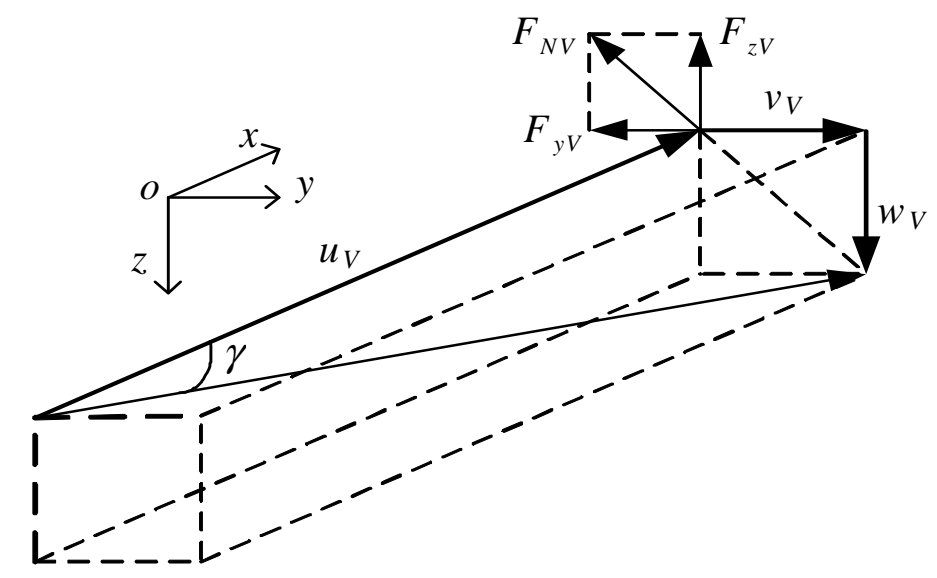

Figure 2.8: Normal force decomposition

over the fin area. If we consider a point $\mathrm{P}$ on the fin planform (shown in Fig. 2.6), with longitudinal position $x$ and spanwise position $s$, the normal force at an area element is predicted as

$$
d F_{F N}=q_{0} \Delta C_{p \alpha}(x, s) \alpha_{e}(x, s) d x d s
$$

where $\Delta C_{p \alpha} \equiv \partial \Delta C_{p} / \partial \alpha$, and $\Delta C_{p}$ is the pressure coefficient of the airfoil; $\Delta C_{p \alpha}$ is determined by the local chordwise position and can be obtained from experiments or from CFD results of the pressure distribution of the airfoil. The effective angle of attack $\alpha_{e}$ is computed as

$$
\alpha_{e}=\frac{C_{L \alpha}}{C_{l \alpha}}\left(1+\frac{R^{2}}{s^{2}}\right) \alpha_{F}
$$

where $C_{L \alpha} / C_{l \alpha}$ is a correction factor for 3-D effects and can be obtained from finite wing theories, such as those in [108]. The factor $\left(1+R^{2} / s^{2}\right)$ accounts for the influence of the hull on the fins [32]. The geometric angle of attack $\alpha_{F}$ employed in Eq. (2.21) is computed from the local velocity at the center of the 1/4-chord line (see Fig. 2.6). Figure 2.9 shows a fin located in the plane inclined at an angle $\Phi_{F}$ from the oxz plane. The velocity component in the $x$ direction at the center of the $1 / 4$-chord is $u(c / 4)$ and the transverse velocity is decomposed into $v_{n}(c / 4)$ perpendicular to the fin surface and $v_{t}(c / 4)$ parallel to the surface. Then the geometric angle of attack is 


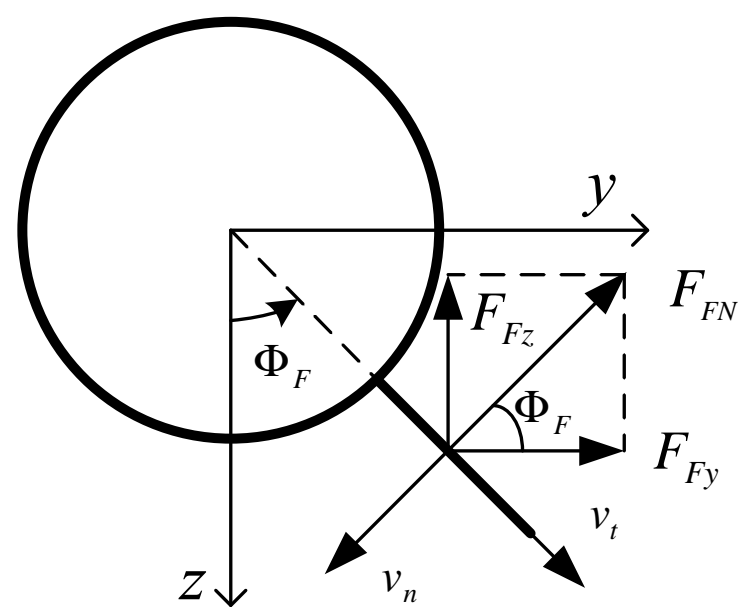

Figure 2.9: Normal force on a fin

computed as

$$
\alpha_{F}=\tan ^{-1}\left[\frac{v_{n}(c / 4)}{u(c / 4)}\right]
$$

If $\alpha_{F}$ is beyond the angle $\alpha_{\text {stall }}$ at which the stall occurs, we use $\alpha_{\text {stall }}$ to calculate the effective angle of attack in Eq. (2.21). The value of $\alpha_{\text {stall }}$ can be predicted by the methods in [107].

The total normal force on the fin is obtained by integrating the force distribution, from Eq. (2.20), over the exposed fin area, i.e.,

$$
\mathbf{F}_{F}=F_{F N}\left[0, \cos \Phi_{F},-\sin \Phi_{F}\right]^{\mathrm{T}}
$$

where

$$
F_{F N}=q_{0} \frac{C_{L \alpha}}{C_{l \alpha}} \alpha_{F} \int_{x_{F s}}^{x_{F e}} \int_{R}^{b} \Delta C_{p \alpha}(x, s)\left(1+\frac{R^{2}}{s^{2}}\right) d s d x
$$

The corresponding rolling, pitching and yawing moments on the fin are obtained as

$$
\mathbf{M}_{F}=\left[M_{F x}, M_{F N} \sin \Phi_{F}, M_{F N} \cos \Phi_{F}\right]^{\mathrm{T}}
$$


where

$$
\begin{aligned}
M_{F x} & =-q_{0} \frac{C_{L \alpha}}{C_{l \alpha}} \alpha_{F} \int_{x_{F s}}^{x_{F e}} \int_{R}^{b} s \Delta C_{p \alpha}(x, s)\left(1+\frac{R^{2}}{s^{2}}\right) d s d x, \\
M_{F N} & =q_{0} \frac{C_{L \alpha}}{C_{l \alpha}} \alpha_{F} \int_{x_{F s}}^{x_{F e}} \int_{R}^{b} x \Delta C_{p \alpha}(x, s)\left(1+\frac{R^{2}}{s^{2}}\right) d s d x
\end{aligned}
$$

Equations. (2.23) and (2.24) can be applied to compute the normal forces and corresponding moments on each fin, which are then added to the right hand side of Eq. (2.2).

\subsubsection{Force acting on the hull due to the fins, normal to the centerline}

Based on the results from wind-tunnel tests on the aerodynamics of model airships, such as the Akron $[45,47]$, it has been found that the presence of the fins can lead to an additional normal force on the hull, due to the fin-induced downwash over the airflow near the hull. The extra normal force per unit length on the hull can be obtained as $[31]$ :

$$
f_{H(F)}=\pi \rho R^{2} V \frac{d w_{d F}}{d x}
$$

where $V$ is the air speed, $w_{d F}$ is the local fin-induced downwash. The variation of the downwash along the centerline can be computed from the force distribution on the fins [109], as given by Eq. (2.20), and we have

$$
w_{d F}(x)=V \frac{C_{L \alpha}}{C_{l \alpha}} \alpha_{F} \int_{x_{F s}}^{x_{F e}} \int_{R}^{b}\left[8 \pi d_{F}\left(d_{F}-x+x_{F}\right)\right]^{-1} \Delta C_{p \alpha}\left(x_{F}, s\right)\left(1+\frac{R^{2}}{s^{2}}\right) d s d x_{F}
$$

where $d_{F}=\sqrt{\left(x-x_{F}\right)^{2}+s^{2}}$ is the distance from a point on a fin to a point on the centerline. Thus, Eqs. (2.26) with (2.27) can be applied to calculate the fin-induced normal force on the hull and the corresponding pitching and yawing moments, which are then added to the right hand side of Eq. (2.2). 


\subsubsection{Axial drag}

The axial force consists of two components, the contributions from the hull and the fins, respectively. At low angles of attack, these forces can be obtained as

$$
\mathbf{F}_{A x i a l, H}=\left[-q_{0} C_{D H 0} S_{H} \cos ^{2} \alpha, 0,0\right]^{\mathrm{T}}, \quad \mathbf{F}_{A x i a l, F}=\left[-q_{0} C_{D F 0} S_{F} \cos ^{2} \alpha_{F}, 0,0\right]^{\mathrm{T}}
$$

where the angle of attack of the hull $\alpha$ is computed from the local velocity at the C.V., $C_{D H 0}$ and $C_{D F 0}$ are the zero-angle axial drag coefficients of the hull and the fins respectively, and $S_{H}$ and $S_{F}$ are the corresponding reference areas. For example, these drag coefficients can be obtained from [108]. To incorporate the axial drag, the forces from Eq. (2.28) are added to the right hand side of Eq. (2.2).

\subsubsection{Force and moment due to control surface deflection}

The force and moment due to the control surface deflections also need to be predicted. A prediction method for the effects of flap deflection on the aerodynamics of a 2-D airfoil section is given in [108], and this is now extended to 3-D fins. That is, the lift coefficient from the deflection of control surface can be computed as

$$
\Delta C_{L}=C_{L \alpha} \tau \eta_{d} k_{3 D} \delta
$$

where $\delta$ is the deflection angle of the control surface and $C_{L \alpha}$ is the 3 -D lift curve slope. The theoretical effectiveness factor $\tau$ is derived from potential flow theory as [108]

$$
\tau=1-\frac{\theta_{f}-\sin \theta_{f}}{\pi}
$$

where $\theta_{f}=\cos ^{-1}\left(2 c_{f} / c-1\right)$, in which $c_{f}$ and $c$ are the flap chord and airfoil chord respectively. The correction factor $\eta_{d}$ is based on experiments and given as a function of $\delta$ for plain flap, as plotted in Fig. 2.10. An efficiency factor $k_{3 D}$ is used to account 


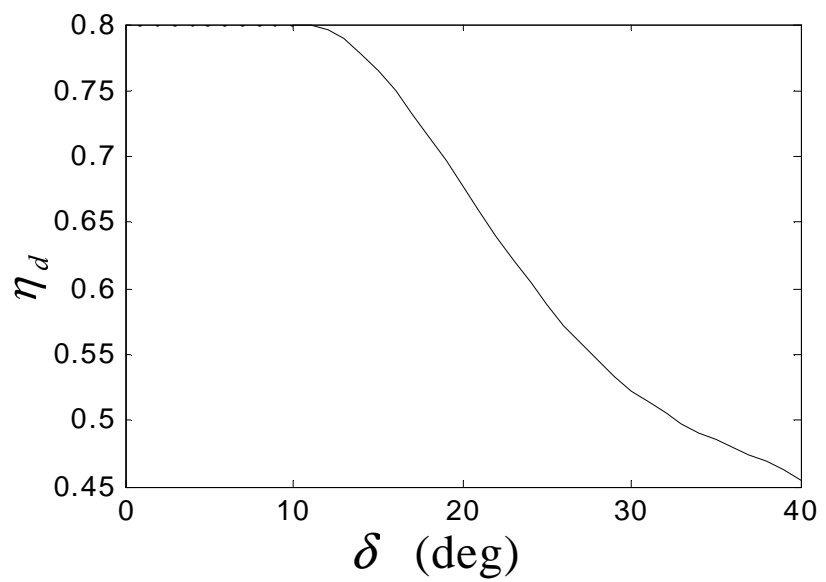

Figure 2.10: $\eta_{d}$ for plain flap

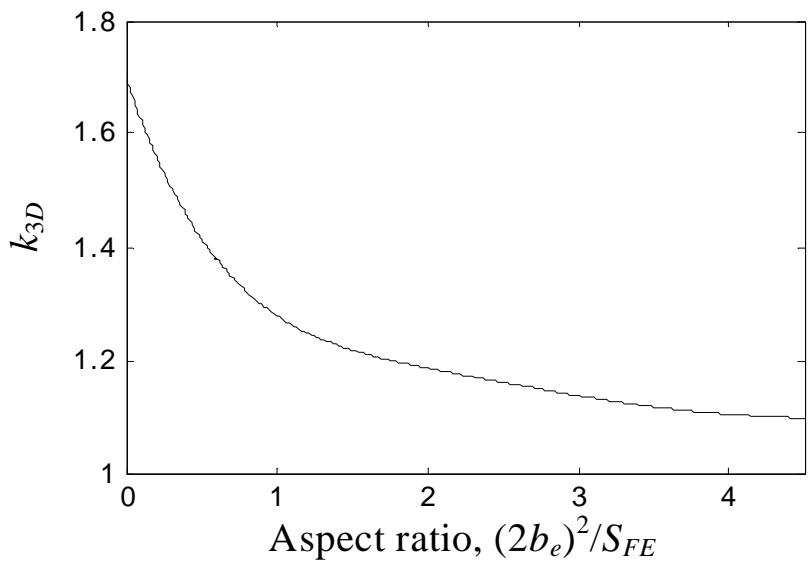

Figure 2.11: 3-D Effectiveness factor for $\tau=0.5$

for 3-D effects and is a function of $\tau$ and aspect ratio [107]; for example, Fig. 2.11 shows $k_{3 D}$ for the case where $\tau=0.5$.

The increment in the drag coefficient, $\Delta C_{D}$, due to the flap deflection is given approximately for plain flaps as [108]

$$
\Delta C_{D}=1.7\left(\frac{c_{f}}{c}\right)^{1.38}\left(\frac{S_{f}}{S_{F E}}\right) \sin ^{2} \delta
$$

where $S_{f}$ is the fin flap area. The influence of flap deflection on the pitching moment coefficient can be estimated from thin airfoil theory, i.e., the ratio of $\Delta C_{M 1 / 4}$ to $\Delta C_{L}$ 
is written as [108]

$$
\frac{\Delta C_{M 1 / 4}}{\Delta C_{L}}=-\frac{2 \sin \theta_{f}-\sin 2 \theta_{f}}{8\left(\pi-\theta_{f}+\sin \theta_{f}\right)}
$$

where $\Delta C_{M 1 / 4}$ is the pitch moment coefficient about the $1 / 4$-chord.

The forces and moments due to the rudder and elevator deflections can be predicted using Eqs. (2.29), (2.31), and (2.32), and then added to the right hand side of Eq. (2.2).

\subsection{Linearized Model}

A linear dynamics model is now formulated to allow a quantitative assessment of the flight stability and the frequency responses to control inputs of the airship. Although analytical linear dynamics models have been used to study airship stability, such as [23], the aerodynamic loads in these models are usually written in terms of aerodynamic derivatives. However, the aerodynamic forces and moments in the present work are expressed nonlinearly in terms of the linear and angular velocities, and it is intractable to obtain an analytical linear formulation for these forces and moments. Therefore, we use a numerical technique to linearize the airship's dynamics equations of motion. This method has been applied to other nonlinear dynamics models for control design [110], and particularly, it has been previously employed to investigate the stability of a tethered aerostat [111].

The nonlinear dynamics model developed in Sections 2.1 to 2.3 can be written as a set of first-order ordinary differential equations as

$$
\dot{\mathbf{X}}=\mathbf{F}(\mathbf{X}, \mathbf{U})
$$

where $\mathbf{X}$ is the state vector defined as $\mathbf{X}=\left[u_{0}, w_{0}, q, \theta, v_{0}, p, r, \phi\right]^{\mathrm{T}}$, recalling that $\theta$ and $\psi$ are the pitch and yaw angle respectively. The first four states describe the longitudinal motion and the last four describe the lateral motion. $\mathbf{U}$ is the control 
input vector, such as that resulting from the elevator and rudder deflections. Equation (2.33) represents eight nonlinear equations, or, $\mathbf{F}=\left[f_{1}, f_{2}, \cdots, f_{8}\right]^{\mathrm{T}}$. The time rates of change of the linear and angular velocities contained in $\dot{\mathbf{X}}$ are obtained from Eq. (2.2), with the aerostatics and aerodynamics models incorporated. In addition, the rates $\dot{\phi}$ and $\dot{\theta}$ can be computed as follows [9]

$$
\dot{\phi}=p+q \sin \phi \tan \theta+r \cos \phi \tan \theta, \quad \dot{\theta}=q \cos \phi-r \sin \phi
$$

To derive the linear dynamics model, the first step is to introduce a reference equilibrium state $\overline{\mathbf{X}}$, about which the system will be linearized, and the corresponding control $\overline{\mathbf{U}}$. If the airship is in steady level flight at equilibrium, then $\overline{\mathbf{X}}=$ $\left[\bar{u}_{0}, 0,0,0,0,0,0,0\right]^{\mathrm{T}}$, where $\bar{u}_{0}$ is an equilibrium speed. The second step is to write the equations for the small disturbance from equilibrium as

$$
\Delta \dot{\mathbf{X}}=\mathbf{F}(\overline{\mathbf{X}}+\Delta \mathbf{X}, \overline{\mathbf{U}}+\Delta \mathbf{U}) \approx \mathbf{A} \cdot \Delta \mathbf{X}+\mathbf{B} \cdot \Delta \mathbf{U}
$$

where $\mathbf{A}=\partial \mathbf{F} / \partial \mathbf{X}$ is the state matrix, or the Jacobian of $\mathbf{F}$ with respect to $\mathbf{X}$, with a similar definition of the control input matrix $\mathbf{B}=\partial \mathbf{F} / \partial \mathbf{U}$. The matrices $\mathbf{A}$ and $\mathbf{B}$ can be evaluated numerically at $\overline{\mathbf{X}}$ and $\overline{\mathbf{U}}$ by finite difference of the nonlinear differential equations.

Once the matrix A is obtained, the airship's stability is characterized by the eigenvalues and eigenvectors of $\mathbf{A}$. The eigenvalues can be either distinct and real, representing non-oscillatory modes, or complex conjugates as $\lambda_{1,2}=\sigma \pm \mathrm{j} \omega_{d}$, representing oscillatory modes. For an oscillatory mode, the natural frequency and damping ratio can be evaluated from $\sigma$ and $\omega_{d}$. For a stable airship, the real parts of all the eigenvalues must be negative. The eigenvector represents the relationship of the elements of the state variables in the corresponding mode. Each element of an eigenvector denotes the magnitude and phase of the response of a particular state variable relative 
to other states.

\subsection{Numerical Simulation}

The numerical simulation results presented in this section include three parts. First, the added-mass terms and steady-state aerodynamic force estimates are obtained and compared to CFD calculations or wind-tunnel test results. Next, the nonlinear dynamics simulation results for the Skyship-500 airship are shown and the time responses due to control surface deflections are analyzed. These simulated results show a good match to the flight test data of the Skyship-500, even though the correction and efficiency factors employed in Eqs. (2.15) and (2.29) are obtained from the experiments on older airships or other aircraft. Third, the linear dynamics model of the Skyship500 airship is analyzed to evaluate the flight stability and the control responses in the frequency domain.

\subsubsection{Validation of the aerodynamics model}

\subsubsection{Added mass and moment of inertia of the Lotte airship}

CFD packages have been developed to compute the aerodynamics of 3-D bodies in potential flow and these have been applied to the added mass calculation for airships. For example, in [39], a CFD package was applied to compute the added mass and moment of inertia of the Lotte airship, shown in Fig. 2.12(a). This CFD method utilizes a distribution of source density on the body surface and solves for the distribution necessary to meet the boundary conditions. We now use these results to evaluate the prediction method given in Section 2.3.1.2.

The CFD results for the added mass and moment of inertia are listed in Table 2.1 and compared to our estimation results. We can observe that the estimation method 


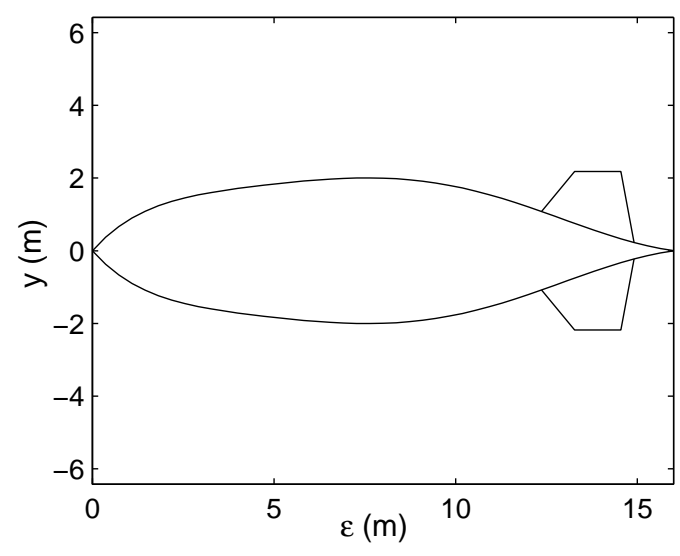

(a) The Lotte airship

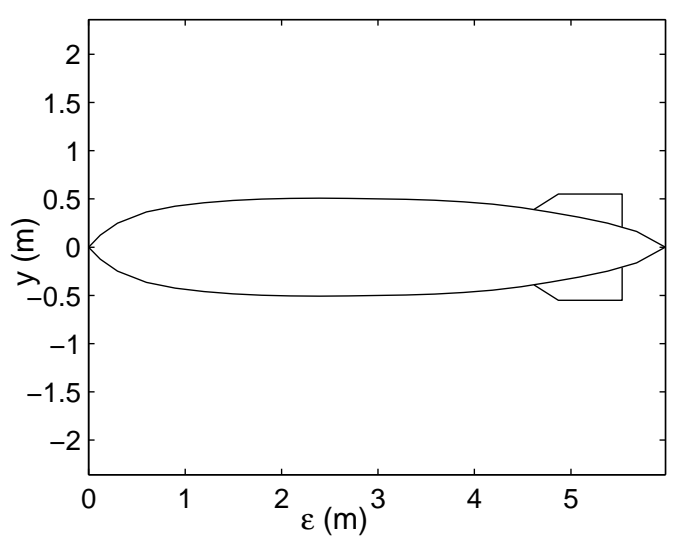

(b) The Akron model

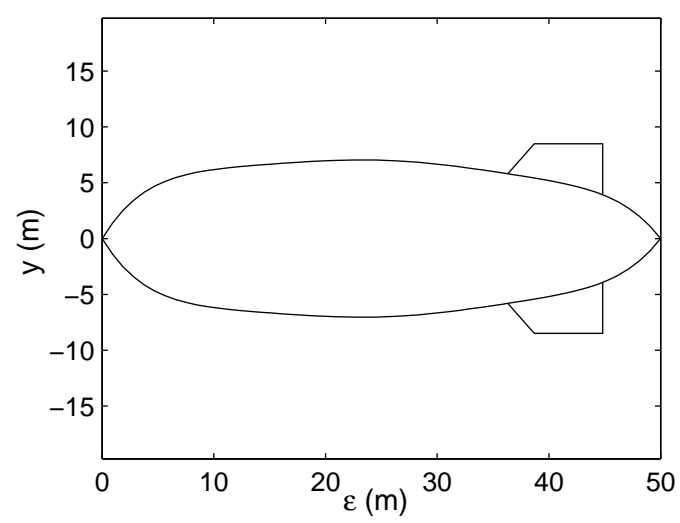

(c) The Skyship-500 airship

Figure 2.12: Profiles of the airships in simulation examples

presented here leads to a reasonable approximation for the added mass of the bare hull and the hull-fin combination. As well, we note that the fins have a considerable effect on the added moment of inertia.

\subsubsection{Steady-state aerodynamics of the Akron airship model}

A great deal of experimental wind tunnel results on the steady-state aerodynamics can be found in the literature for old rigid airships. In this section, the experimental results for the 1/40-scale Akron airship model [45] (drawn in Fig. 2.12(b)) are used to test our aerodynamics computation. The measured normal force and pitching moment (about the C.V.) coefficients at low angles of attack are compared to our prediction results in Figs. 2.13(a) and 2.13(b), for both bare hull and hull-fin combination. We can see 
Table 2.1: Added-mass results

\begin{tabular}{cccccc}
\hline & \multicolumn{2}{c}{ Bare hull } & & \multicolumn{2}{c}{ Hull-fin combination } \\
\cline { 2 - 3 } \cline { 6 - 6 } Added-mass terms & CFD [39] & Prediction & & CFD [39] & Prediction \\
\hline$m_{11}(\mathrm{~kg})$ & 13 & 11 & & 12.7 & 11 \\
$m_{22}(\mathrm{~kg})$ & 112 & 114 & & 129 & 125 \\
$m_{55}\left(\mathrm{~kg} \times \mathrm{m}^{2}\right)$ & 759 & 793 & & 1379 & 1279 \\
\hline
\end{tabular}

that the estimates from Section 2.3 can provide a good match to the experimental results.

\subsubsection{Nonlinear dynamics simulation for the Skyship-500 air- ship}

A dynamic simulation program has been developed in the MATLAB environment to implement the nonlinear dynamics model discussed in Section 2.1 to 2.3. In the numerical simulation, the Skyship-500 airship, shown in Fig. 2.12(c), is used as an example, because the dimensional and inertial parameters, and flight test data are available for this airship $[17,62]$. This subsection contains the simulation results for the transient responses due to elevator or rudder deflection and compares them to flight test results.

The inertial parameters of the Skyship-500 are listed in Table 2.2. To obtain an aerostatic force equal to the gravitational force (neutral buoyancy), the air density must be $\rho=1.158 \mathrm{~kg} / \mathrm{m}^{2}$, which implies that the airship flies at an altitude of about $575 \mathrm{~m}$. The operating speed of this airship is usually below $30 \mathrm{~m} / \mathrm{s}$ [17,62].

\subsubsection{Thrust force}

A thrust force model is needed in order to perform the nonlinear dynamics simulation. Two Porsche six-cylinder internal combustion engines and two Hoffman fine-blade 


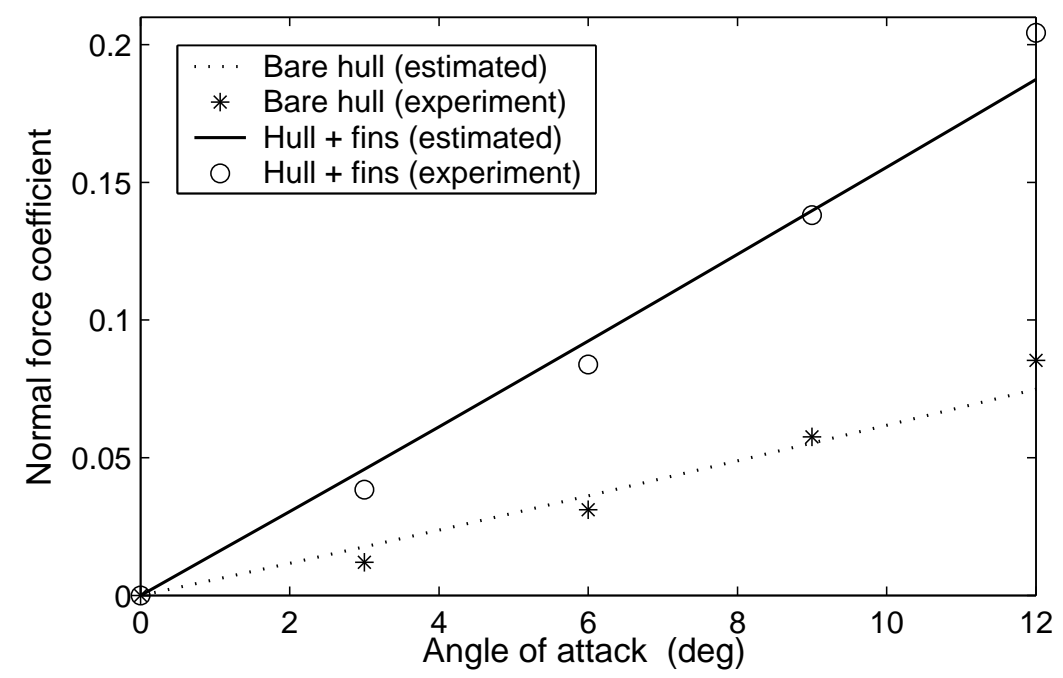

(a) Normal force result

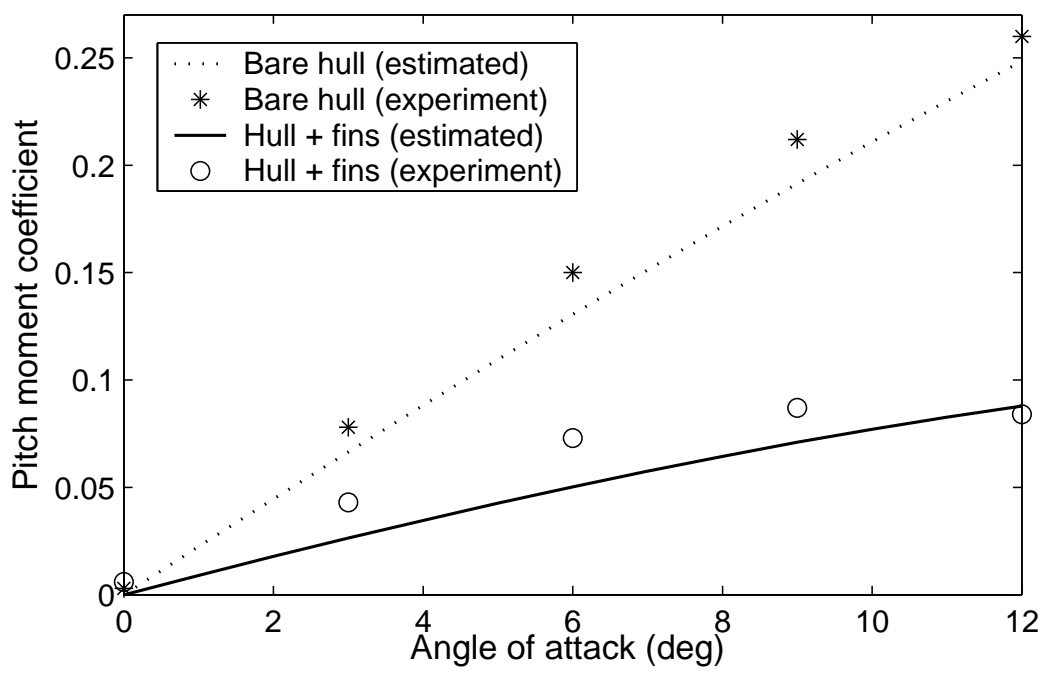

(b) Pitch moment result

Figure 2.13: Steady-state aerodynamics results of the Akron model

Table 2.2: Mass, position of C.G. and moments of inertia (about the C.V.) of the Skyship-500 [17]

\begin{tabular}{cccccc}
\hline$m(\mathrm{~kg})$ & $x_{G}(\mathrm{~m})$ & $z_{G}(\mathrm{~m})$ & $I_{x x}\left(\mathrm{~kg} \times \mathrm{m}^{2}\right)$ & $I_{y y}\left(\mathrm{~kg} \times \mathrm{m}^{2}\right)$ & $I_{z z}\left(\mathrm{~kg} \times \mathrm{m}^{2}\right)$ \\
\hline 5942 & 0 & 5.1816 & 200658 & 850900 & 649699 \\
\hline
\end{tabular}


propellers are used in the thrusters of the Skyship-500 [61]. The thrust force $F_{T}$ can be written as a proportional function of the input to the engine:

$$
F_{T}=k_{T} \delta_{T}
$$

where $\delta_{T}$ is the displacement of throttle crank, a mechanism that regulates the amount of air entering the engine. The factor $k_{T}$ is obtained from the acceleration test results in [61]. In this test, the airship was hovering initially and started to accelerate once the throttle crank input was given. The airspeed of the airship was measured during the flight.

This acceleration maneuver was simulated using a 1-DOF simplified dynamics model of the airship in forward flight. The value of $k_{T}$ was obtained by trial and error to achieve a match between the simulated airspeed, resulting in $k_{T}=-90,000 \mathrm{~N} / \mathrm{m}$. The input of the throttle crank and the airspeed results are displayed in Fig. 2.14. We observe that the proportional thrust force model in Eq. (2.36) leads to reasonable estimates of the airspeed in the acceleration test. It is also noted that a dynamic model for $F_{T}$ was attempted (first- and second-order) with no improvement in the match of the experimental results.

The moment due to each thruster is then obtained as

$$
\mathbf{M}_{T}=\mathbf{r}_{T}^{\times} \mathbf{F}_{T}
$$

where $\mathbf{F}_{T}=\left[F_{T}, 0,0\right]^{\mathrm{T}}$ and $\mathbf{r}_{T}$ is the position vector from the body-frame origin to the point at which the thruster is mounted.

\subsubsection{Responses due to elevator deflection}

In order to predict the responses from the dynamic simulation program, a control force and a control moment are first applied in the simulation so that the airship 

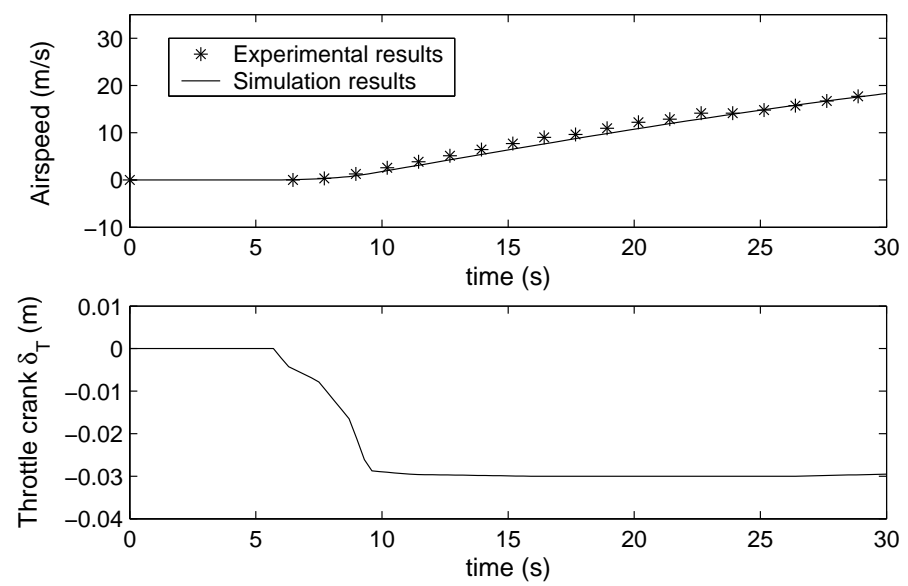

Figure 2.14: Throttle crank input and airspeed in the acceleration test

begins its flight in a trim condition at a constant airspeed of $12.86 \mathrm{~m} / \mathrm{s}$ ( 25 knots).

In this example, the elevator deflection input $\delta_{E}$ (positive trailing edge downward), shown in Fig. 2.15(a), is obtained from the flight test [17]. The resulting time histories of the pitch rate from the simulation and the flight test [17] are compared in Fig. 2.15(b), where we find that the simulated response is very close to the flight test data.

\subsubsection{Responses due to rudder deflection}

In this example, the step-like rudder input $\delta_{R}$ (positive trailing edge left) is plotted in Fig. 2.16(a), and the response results of yaw rate are compared to the flight test data [17] in Fig. 2.16(b). A proportional controller is applied to the thrust input so that the airship maintains a constant airspeed. We can see that the predicted steadystate yaw rate is higher than the test data by about $20 \%$, but generally speaking, the dynamic simulation program provides a reasonable match to the major trends observed in the flight test. 


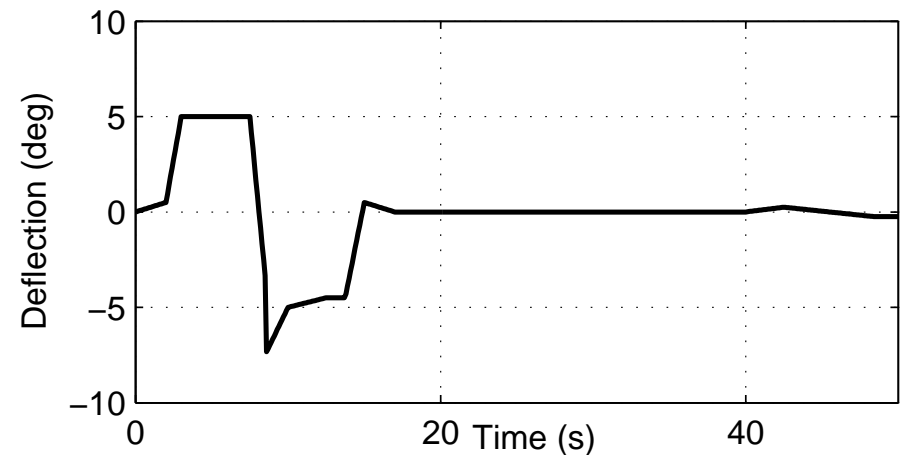

(a) Elevator deflection $\delta_{E}$

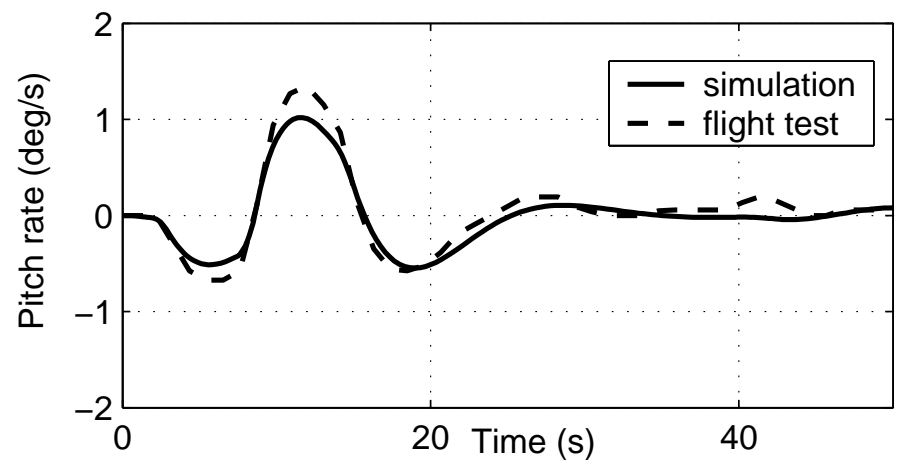

(b) Time history of pitch rate $q$

Figure 2.15: Elevator input and response results at $12.86 \mathrm{~m} / \mathrm{s}$

\subsubsection{Yaw rate in steady turn}

A steady rudder deflection leads to a steady turn flight for the airship. In this example, the yaw rates in steady turns are computed for various rudder deflection angles (from -30 to $30 \mathrm{deg}$ ) at $12.86 \mathrm{~m} / \mathrm{s}$. The simulated results are plotted and compared to the flight test results [62] in Fig. 2.17. We can see that the turn rate is a nonlinear function of the rudder deflection predicted by the dynamics simulation program. 


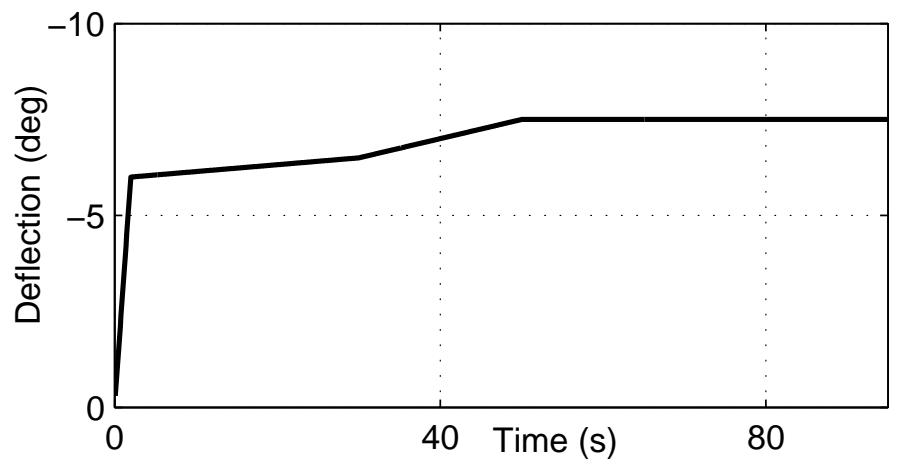

(a) Rudder deflection $\delta_{R}$

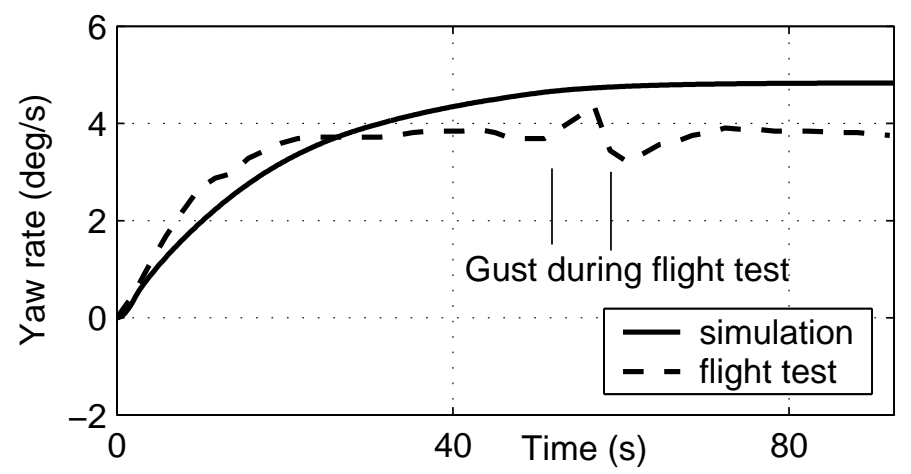

(b) Time history of yaw rate $r$

Figure 2.16: Rudder input and response results at $12.86 \mathrm{~m} / \mathrm{s}$

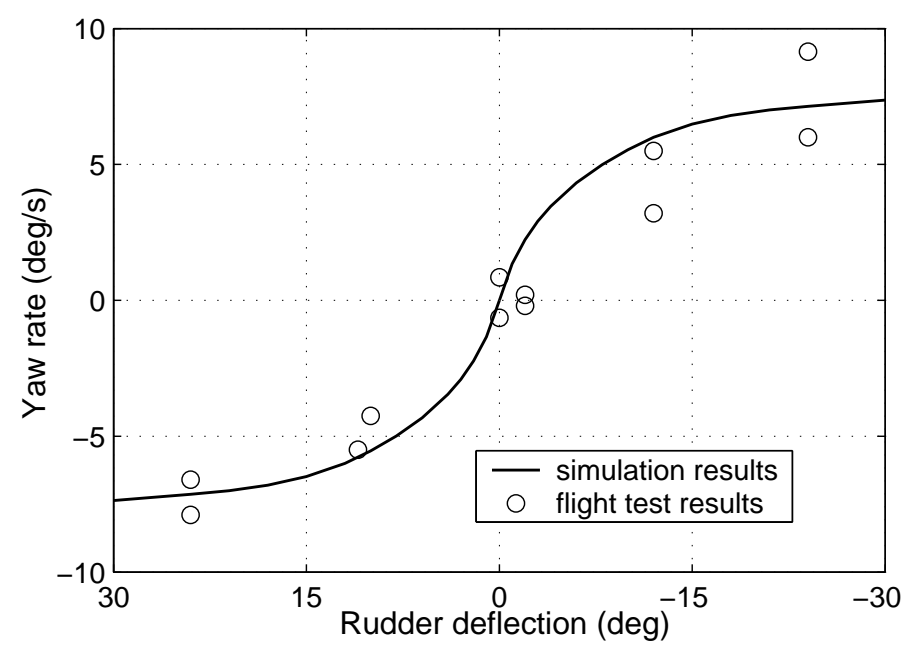

Figure 2.17: Yaw rate in steady turn (at $12.86 \mathrm{~m} / \mathrm{s}$ ) 


\subsubsection{Linearized model and stability analysis for the Skyship- 500 airship}

\subsubsection{Frequency domain response}

The nonlinear dynamics model is linearized for the Skyship-500 airship using the approach described in Section 2.4. It is found that the state matrix A can be partitioned into four distinct submatrices as

$$
\mathbf{A}=\left[\begin{array}{cc}
\mathbf{A}_{\text {long }} & O(\mathbf{0}) \\
\mathbf{0} & \mathbf{A}_{\text {lat }}
\end{array}\right]
$$

where all elements in the lower left submatrix are zero, while some in the upper right submatrix are not exactly zero but of much smaller magnitude than the elements in the $4 \times 4$ matrices $\mathbf{A}_{\text {long }}$ and $\mathbf{A}_{\text {lat }}$. Therefore the longitudinal and lateral motions are essentially decoupled.

Once the matrices $\mathbf{A}$ and $\mathbf{B}$ in Eq. (2.35) are obtained, the control responses in the frequency domain can be computed. The responses due to elevator and rudder inputs are displayed in Bode plots in Figs. 2.18 and 2.19 for forward speeds $\bar{u}_{0}$ of $12.86 \mathrm{~m} / \mathrm{s}$ (25 knots) and $20.58 \mathrm{~m} / \mathrm{s}$ (40 knots), respectively. The responses predicted herein are compared to the flight test results. We can see that the linear model presented herein provides a good match to the major trends in the flight test. In addition, the simulated responses are compared to those predicted by Jex and Gelhausen [17]. The aerodynamic forces and moments in [17] were calculated using the HLASIM simulation program and this program was first applied to a hybrid heavy-lift airship. The comparison in Figs. 2.18 and 2.19 shows that the linear model proposed in this work leads to more accurate predictions than Jex's model results for the yaw rate phase angle response at low frequencies. 

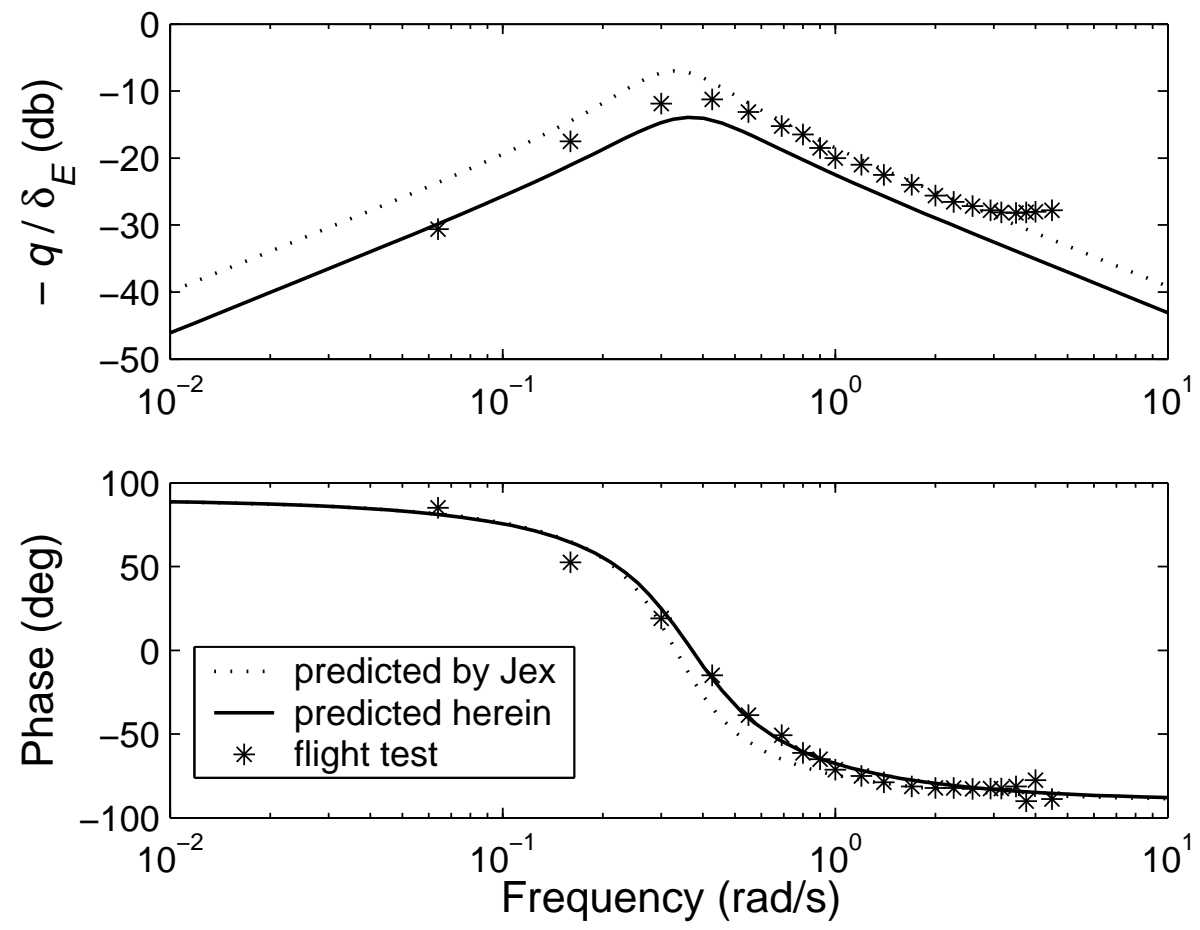

(a) Pitch rate response due to elevator input
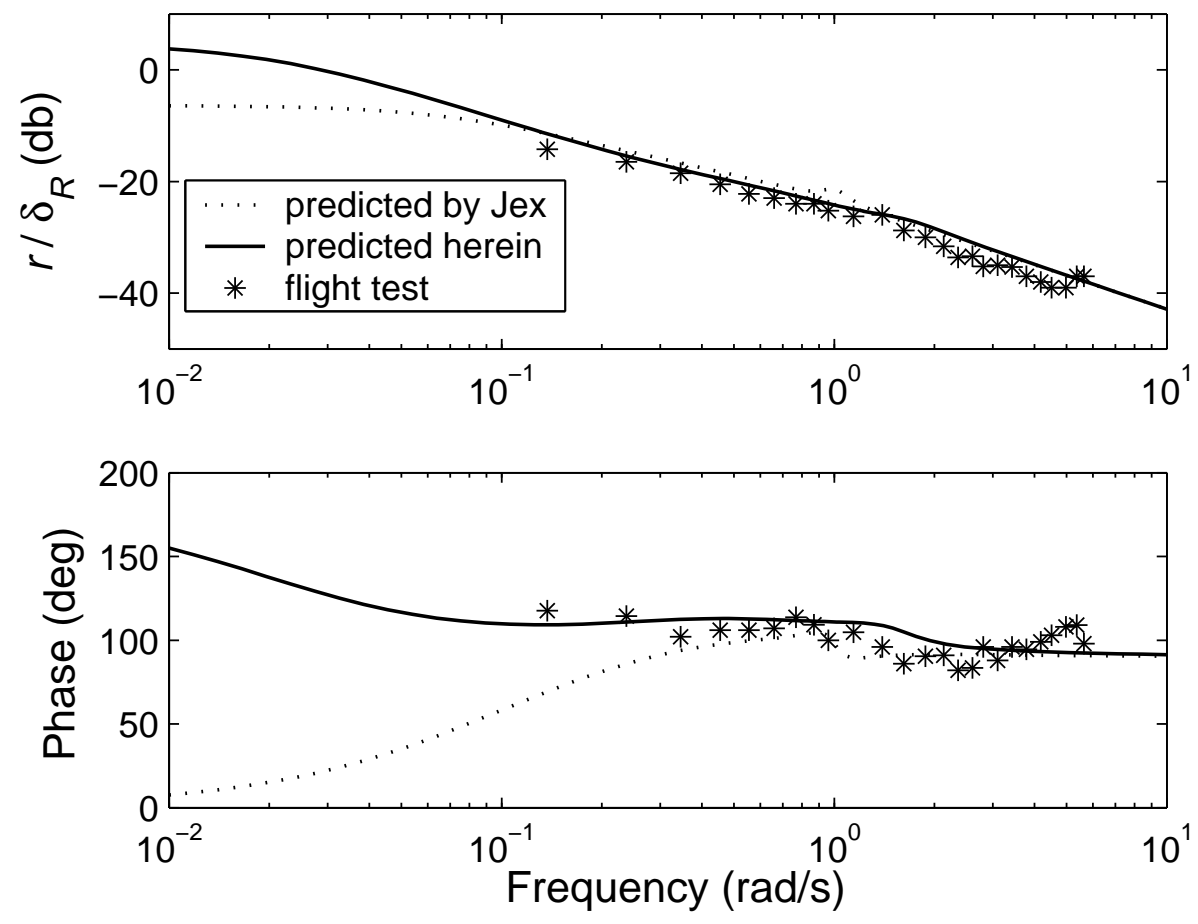

(b) Yaw rate response due to rudder input

Figure 2.18: Control response in the frequency domain at $12.86 \mathrm{~m} / \mathrm{s}$ 

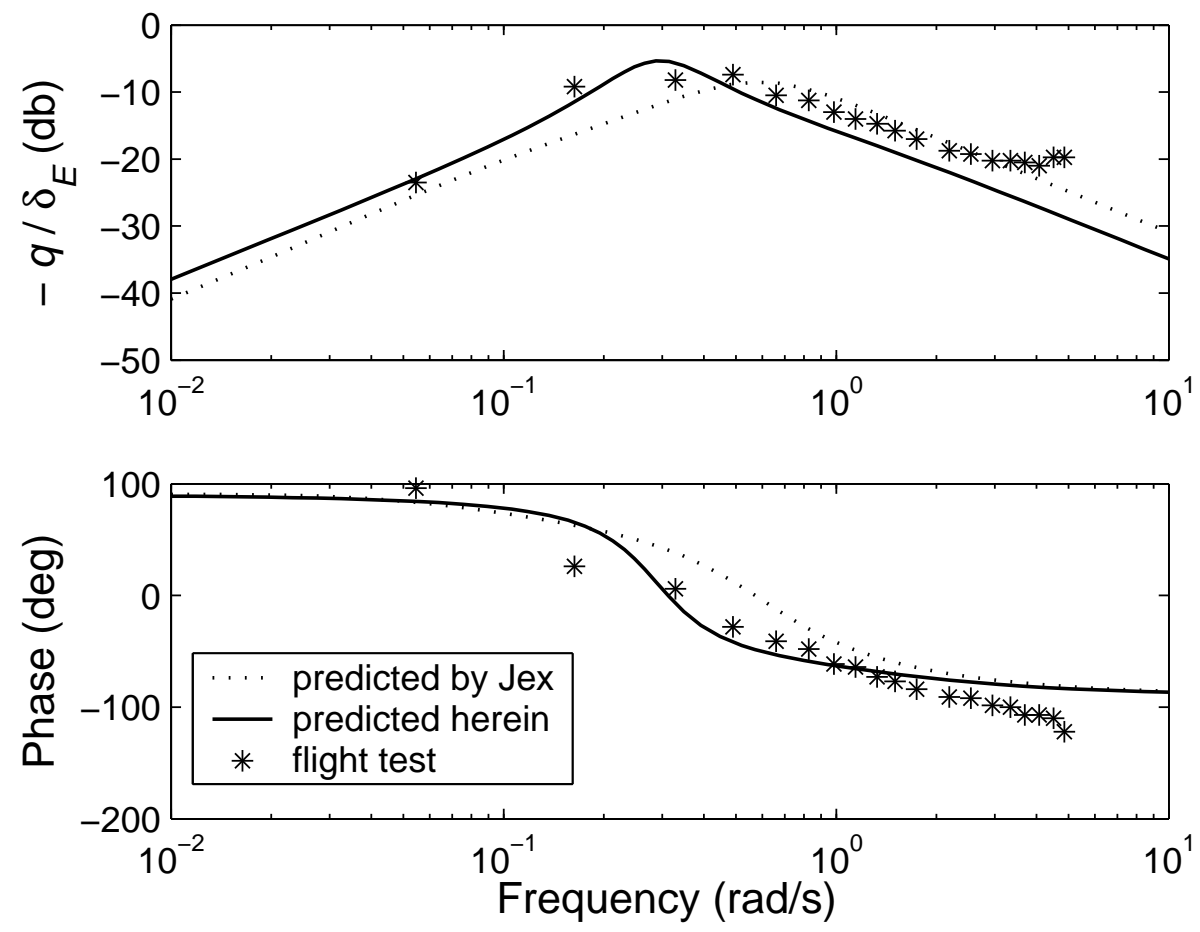

(a) Pitch rate response due to elevator input
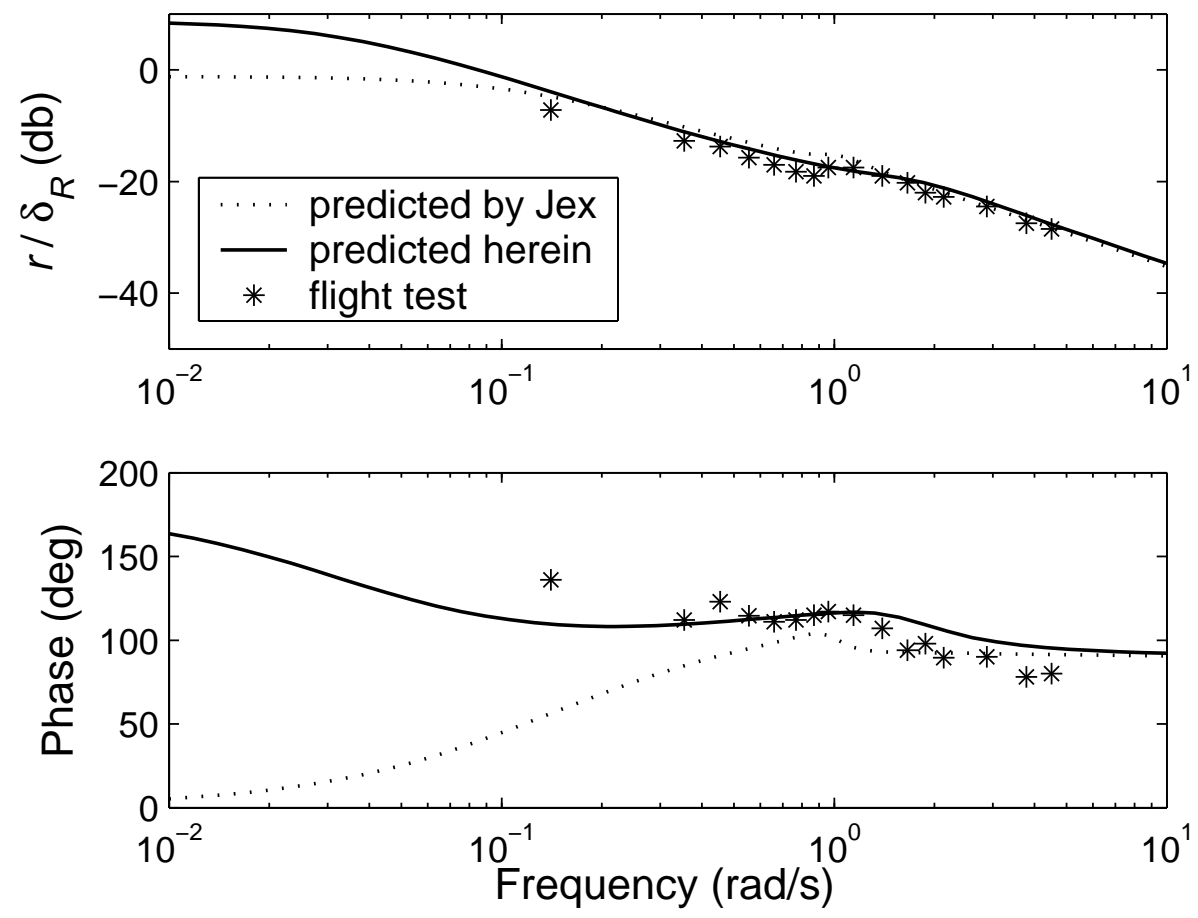

(b) Yaw rate response due to rudder input

Figure 2.19: Control response in the frequency domain at $20.58 \mathrm{~m} / \mathrm{s}$ 


\subsubsection{Stability and natural modes}

The eigenvalues and eigenvectors of $\mathbf{A}_{\text {long }}$ and $\mathbf{A}_{\text {lat }}$ for the Skyship-500 airship are investigated in Figs. 2.20 to 2.27 as they change with variations in the equilibrium speed from 0 to $30 \mathrm{~m} / \mathrm{s}$.

The first two longitudinal modes have negative real eigenvalues, whose magnitude increases with speed (i.e., they become more stable) as shown in Fig. 2.20(a). The eigenvalues of the third longitudinal mode are a complex conjugate pair and the corresponding natural frequency and damping ratio are plotted in Fig. 2.20(b). The eigenvectors are then studied in order to obtain the relative values (magnitude and phase) between the different state variables of each mode, using the following steps. First, we nondimensionalize each component of the eigenvector, i.e., each linear velocity component $\left(\Delta u_{0}, \Delta v_{0}, \Delta w_{0}\right)$ is divided by the equilibrium speed $\bar{u}_{0}$, each angular velocity component $(\Delta p, \Delta q, \Delta r)$ is divided by $\bar{u}_{0} /\left(V_{B}\right)^{1 / 3}$ where $V_{B}$ is the volume of the airship and $\left(V_{B}\right)^{1 / 3}$ is a reference length, and the rotation components, $\Delta \theta$ and $\Delta \phi$, are not modified. Second, we select a reference state variable and factor the eigenvector to make this state equal to unity. Third, all the real and imaginary parts of each component are plotted in an Argand diagram. For eigenvalues that form a complex conjugate pair $\lambda_{1,2}=\sigma \pm \mathrm{j} \omega_{d}$, only the eigenvector corresponding to $\sigma+\mathrm{j} \omega_{d}$ is plotted. For example, the vector diagrams of the longitudinal modes at $1 \mathrm{~m} / \mathrm{s}, 15 \mathrm{~m} / \mathrm{s}$ and $30 \mathrm{~m} / \mathrm{s}$ are shown in Figs. 2.21 to 2.23 respectively.

From the above, we conclude that all longitudinal modes are stable. It is noted that the mode shapes change dramatically with forward speed because the aerodynamic force and moment are negligible at a very low speed but become more important as the speed increases. The particular motion characteristics of each mode at different speeds have similar trends to the modal results of other airships considered by Cook et al. [23] and Kornienko [63]. They can be described as follows:

1) The first longitudinal mode is a surge subsidence mode caused by the axial 


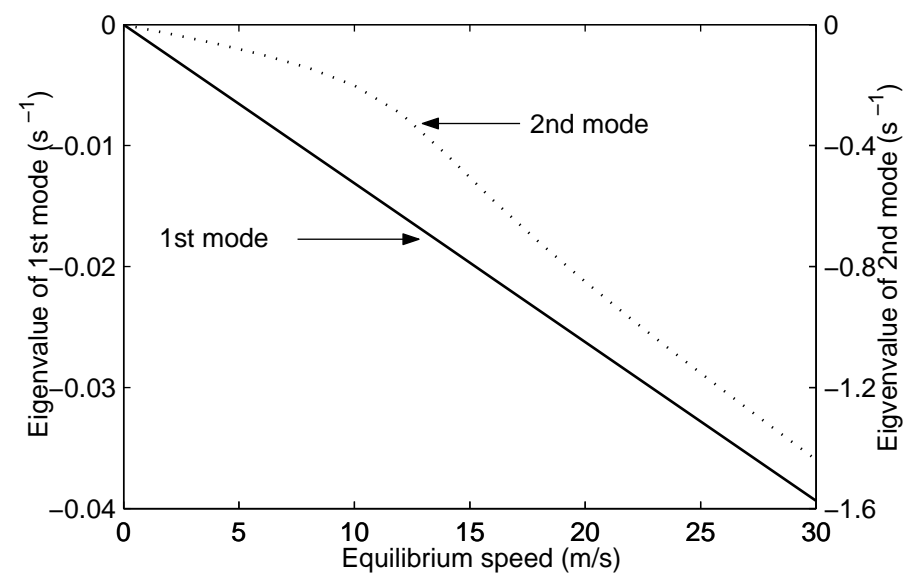

(a) Longitudinal modes (1st and 2nd)
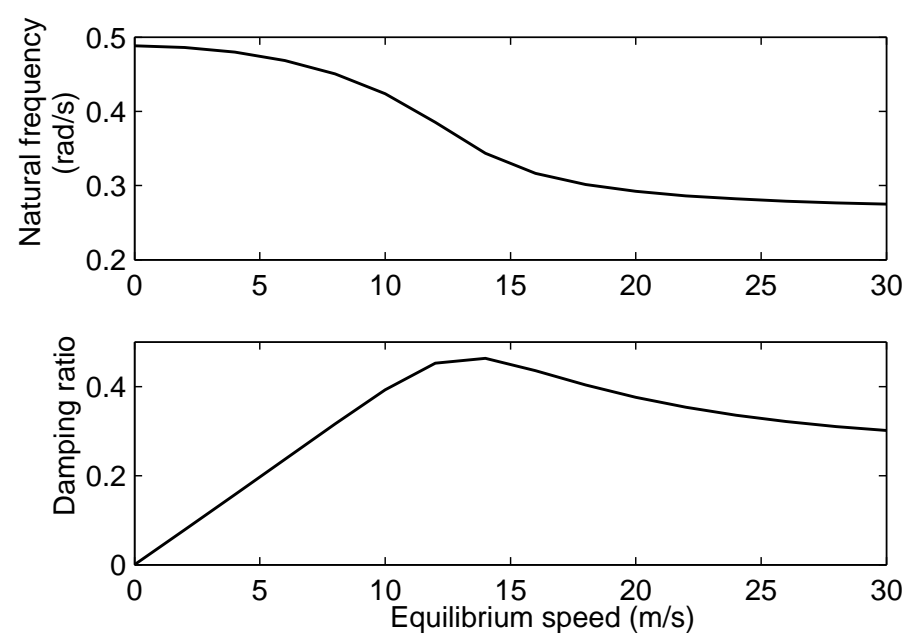

(b) Longitudinal mode (3rd)

Figure 2.20: Longitudinal modes
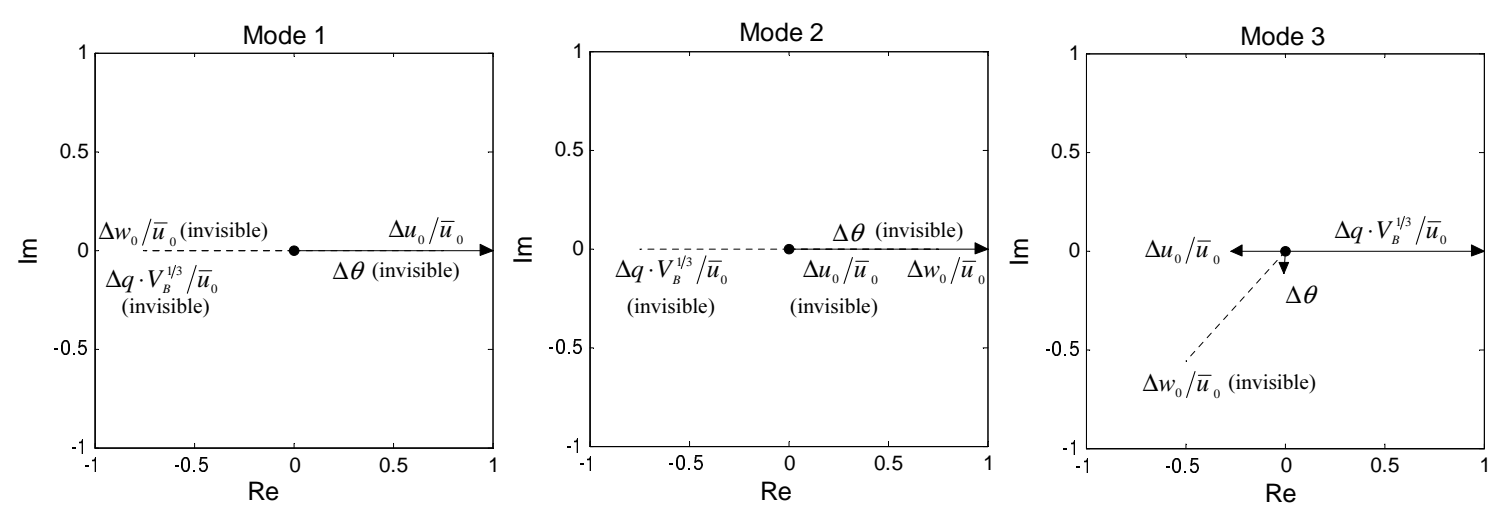

Figure 2.21: Vector diagram of longitudinal modes at $1 \mathrm{~m} / \mathrm{s}$ 

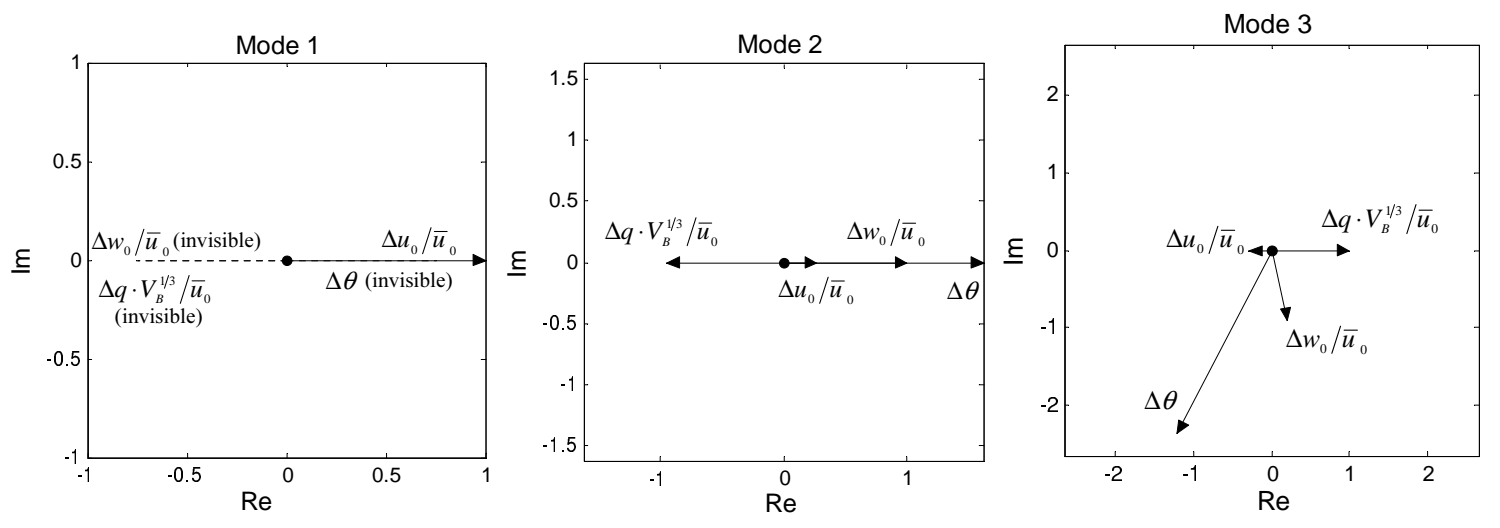

Figure 2.22: Vector diagram of longitudinal modes at $15 \mathrm{~m} / \mathrm{s}$
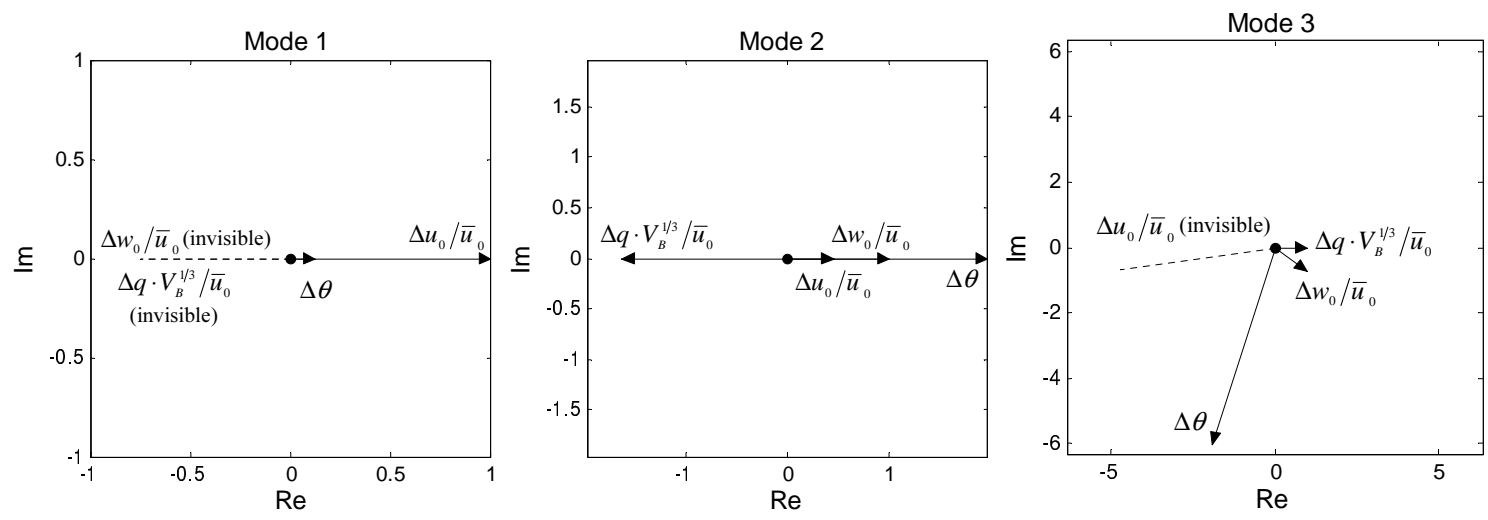

Figure 2.23: Vector diagram of longitudinal modes at $30 \mathrm{~m} / \mathrm{s}$

aerodynamic drag, and can be considered as a 1-DOF motion in forward velocity $\Delta u_{0}$. The modes are neutrally stable at zero speed and become more stable as the speed increases.

2) The second longitudinal mode is a heave-pitch subsidence mode caused by the transverse aerodynamic drag. The dominant motion is $\Delta w_{0}$ near zero speed, coupling with some $\Delta \theta, \Delta q$ and $\Delta u_{0}$ as the speed increases to about $12 \mathrm{~m} / \mathrm{s}$. The pitch rate generates additional aerodynamic damping moment, thus further stabilizing this mode.

3) The third longitudinal mode is a pitch-incidence oscillatory mode. Near zero speed, the dominant motion is the pitch rate $\Delta q$, combined with some $\Delta u_{0}$. As the speed increases to about $14 \mathrm{~m} / \mathrm{s}, \Delta w_{0}$ becomes apparent and the incidence causes a Munk moment, which tends to destabilize the system, thereby leading to a decrease 
in the damping ratio at speeds from $14 \mathrm{~m} / \mathrm{s}$ to $30 \mathrm{~m} / \mathrm{s}$.

The lateral stability modes can be studied using a similar approach. The first two lateral modes have negative real eigenvalues as shown in Fig. 2.24(a). The eigenvalues of the third lateral mode are a complex conjugate pair and the corresponding natural frequency and damping ratio are plotted in Fig. 2.24(b). The corresponding vector diagrams of the lateral modes at $1 \mathrm{~m} / \mathrm{s}, 15 \mathrm{~m} / \mathrm{s}$ and $30 \mathrm{~m} / \mathrm{s}$ are shown in Figs. 2.25 to 2.27 respectively. Again, the lateral modal results are similar to those of the airships in [23] and [63]. All modes are stable, and the motion characteristics of each mode can be described as follows:

1) The first lateral mode is a sideslip-yaw subsidence mode. Near zero speed, the most apparent motion is $\Delta v_{0}$ and yaw rate $\Delta r$. As the speed increases, the roll rotation $\Delta \phi$ becomes apparent because of the centrifugal force.

2) The second lateral mode is a yaw-roll subsidence mode. The yaw rate $\Delta r$ couples with $\Delta v_{0}$ at near-zero speed. This mode becomes slightly less stable as the speed increases from $25 \mathrm{~m} / \mathrm{s}$ to $30 \mathrm{~m} / \mathrm{s}$. This is likely due to the unstable effect of the Munk moment, as in the longitudinal modal analysis.

3) The third lateral mode is a roll oscillatory mode, coupling with $\Delta v_{0}$, and with $\Delta r$ at high speed. The damping ratio curve shows that the aerodynamic damping becomes more significant as the speed increases.

\section{$2.6 \quad 2-D O F$ Model for Steady Turns}

The 6-DOF dynamics simulation model in the above sections can be applied to analyze the dynamic flight behavior of airships, as well as their steady state behavior such as the steady turn rates discussed in Section 2.5.2.4. To perform this analysis, the geometric dimensions and inertial properties (mass, position of C.G. and inertia tensor) must be provided. However, some of these parameters, such as the second moment 


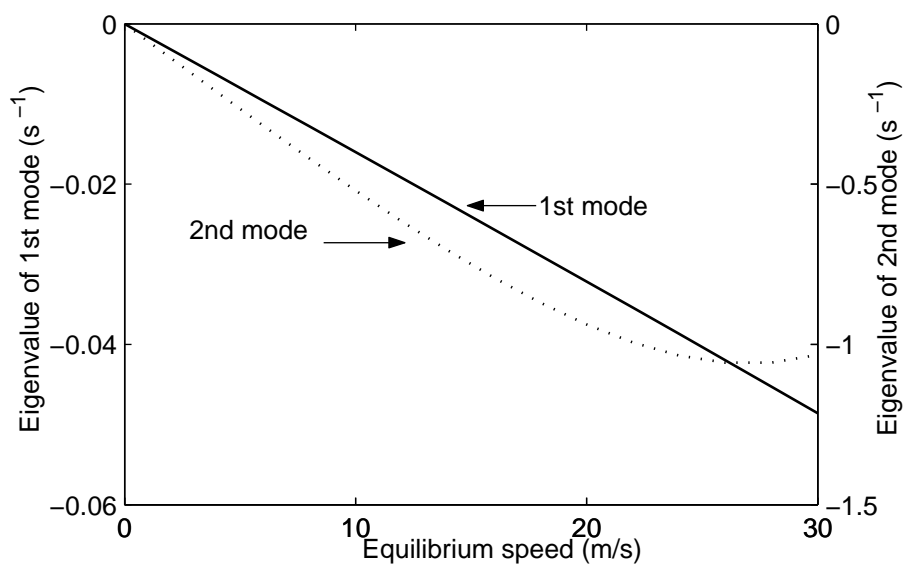

(a) Lateral modes (1st and 2nd)
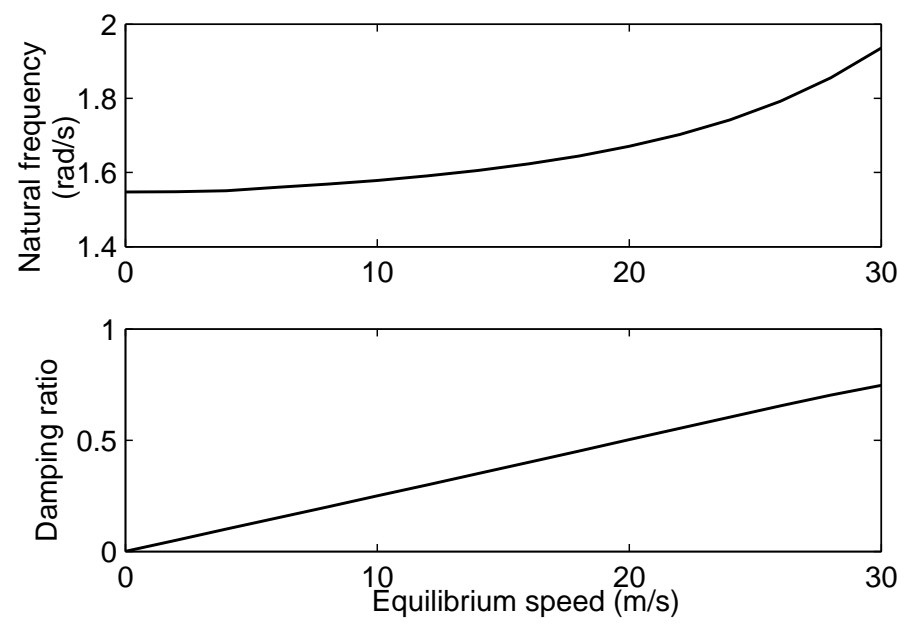

(b) Lateral mode (3rd)

Figure 2.24: Lateral modes
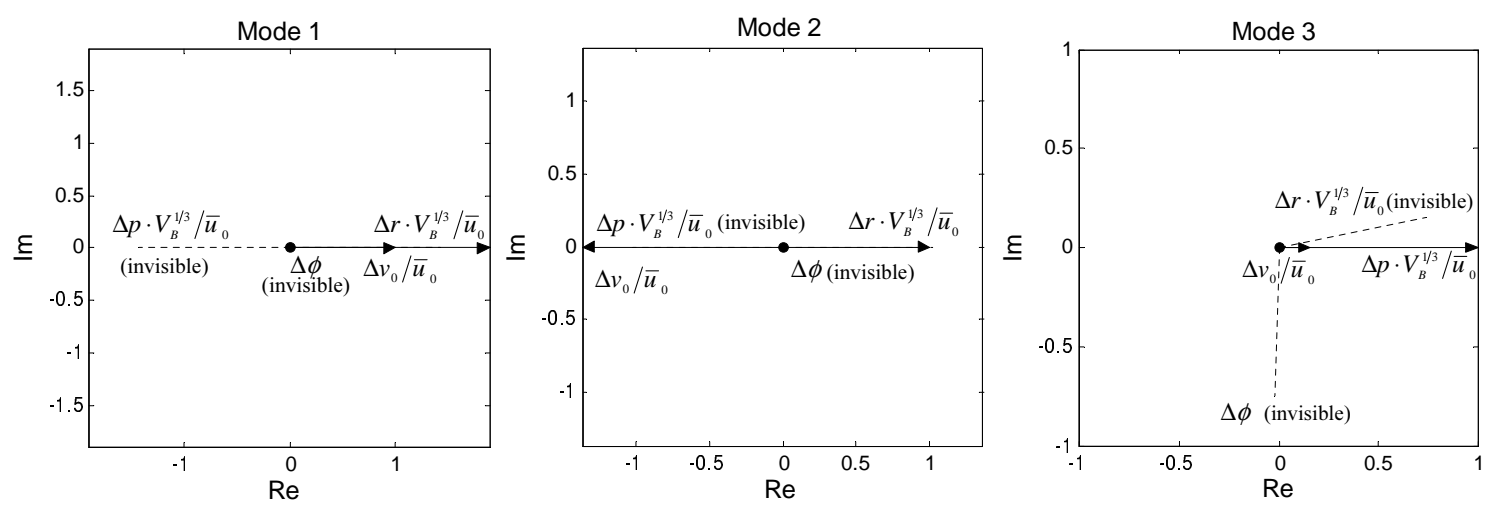

Figure 2.25: Vector diagram of lateral modes at $1 \mathrm{~m} / \mathrm{s}$ 

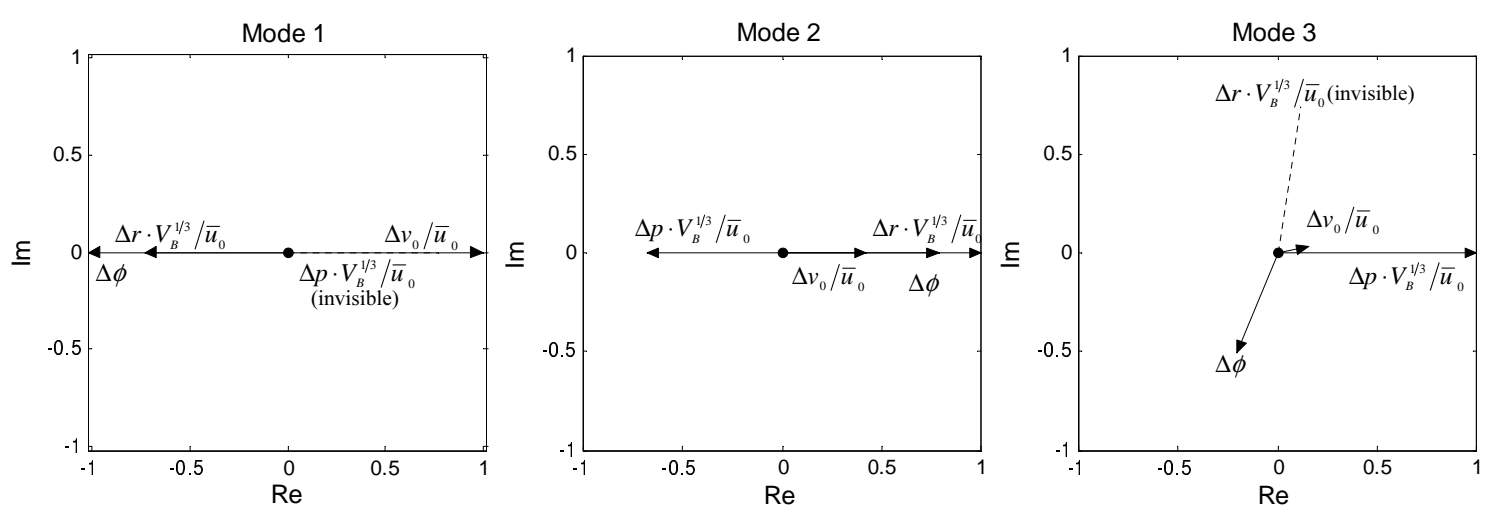

Figure 2.26: Vector diagram of lateral modes at $15 \mathrm{~m} / \mathrm{s}$
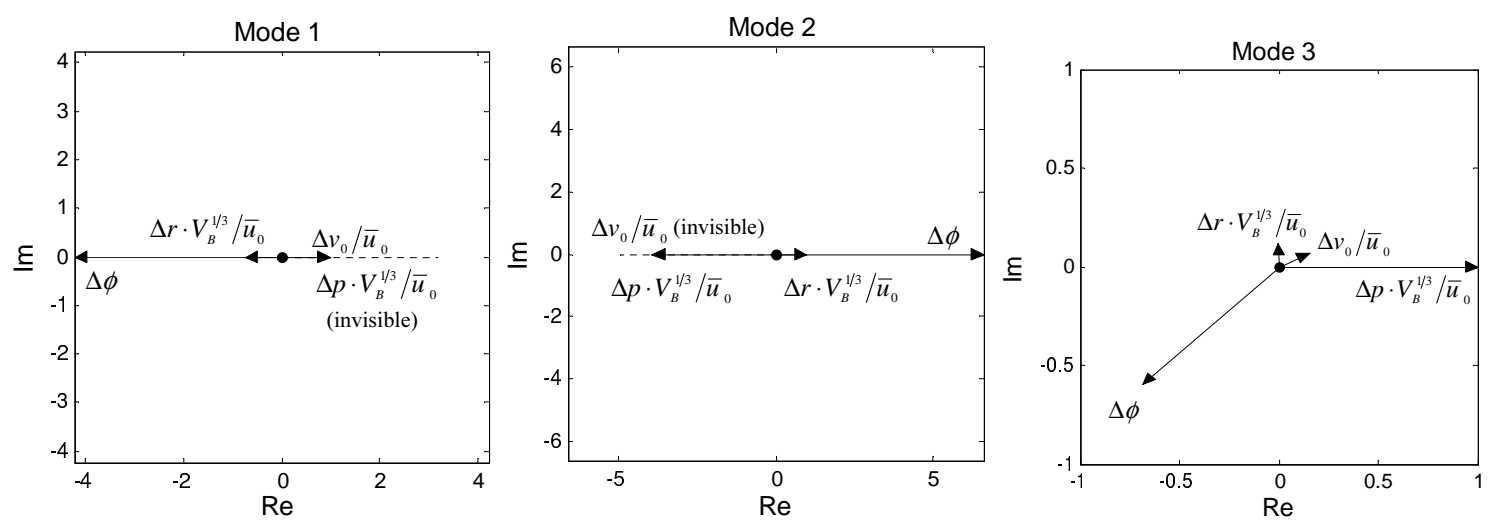

Figure 2.27: Vector diagram of lateral modes at $30 \mathrm{~m} / \mathrm{s}$

of inertia, are not usually provided as part of airship design and are difficult to estimate and may not be needed to evaluate the airship's steady state performance. This section presents a 2-DOF simplified model to predict the steady turn characteristics, which requires much less information than the above 6-DOF model.

\subsubsection{Simplified model}

An airship in a steady turn is shown in Fig. 2.28, with constant linear velocity $u_{0}$ in the $x$ direction and $v_{0}$ in the $y$ direction and a constant yaw rate $r$. In order to perform this steady turn, two control inputs must be specified: a thrust input to maintain the constant airspeed $V$, and a constant rudder deflection to generate the yaw rotation. The procedure of generating a steady turn from a steady straight flight 
is as follows. The airship with forward speed $u_{0}$ is given a positive rudder deflection $\delta_{R}$, thus generating a control force $F_{\delta, y}$, acting at a position $x_{\delta}$, and leading to a negative yaw moment and a velocity $v_{0}$ in the $y$ direction. This lateral velocity leads to two kinds of aerodynamic effects: (1) an unstable negative added-mass aerodynamic yaw moment $M_{A, z}$ (Munk moment), and (2) three aerodynamic normal forces as discussed in Sections 2.3.2 to 2.3.4, i.e., the viscous effect on the hull $F_{V, y}$, the force on the vertical fins $F_{F, y}$, and the force on the hull due to the fins $F_{H(F), y}$. These latter three forces act at the rear of the airship and result in three positive stable yaw moments $M_{V, z}, M_{F, z}$ and $M_{H(F), z}$. Initially, the sum of the Munk moment and the control moment are greater than the sum of the moments due to the other three normal forces, so the airship starts to turn with a yaw rate $r<0$. The negative yaw rate increases the three aerodynamic forces at the rear and thus increases the resulting positive yaw moments. Once the moment from the rudder, the negative Munk moment and the other three positive aerodynamic moments are in balance, the magnitude of the yaw rate stops increasing and the airship is in a steady turn.

A simplified dynamics model can be obtained for a steady turn in a horizontal plane from the following assumptions:

- $V \approx u_{0}$.

- All accelerations are assumed to be zero.

- The roll rotation can be neglected.

Thus, collecting the terms related to $u_{0}, v_{0}$ and $r$ in the lateral motion equations yields:

$$
\begin{aligned}
& F_{1}=-\rho V_{B} u_{0} r+F_{A D, y}\left(u_{0}, v_{0}, r\right)+F_{\delta, y}\left(u_{0}, v_{0}, r\right)=0 \\
& F_{2}=-\rho V_{B} x_{G} u_{0} r+M_{A D, z}\left(u_{0}, v_{0}, r\right)+M_{\delta, z}\left(u_{0}, v_{0}, r\right)=0
\end{aligned}
$$

where $\rho V_{B}$ represents the mass of a neutrally buoyant airship, the subscripts $A D$ and 


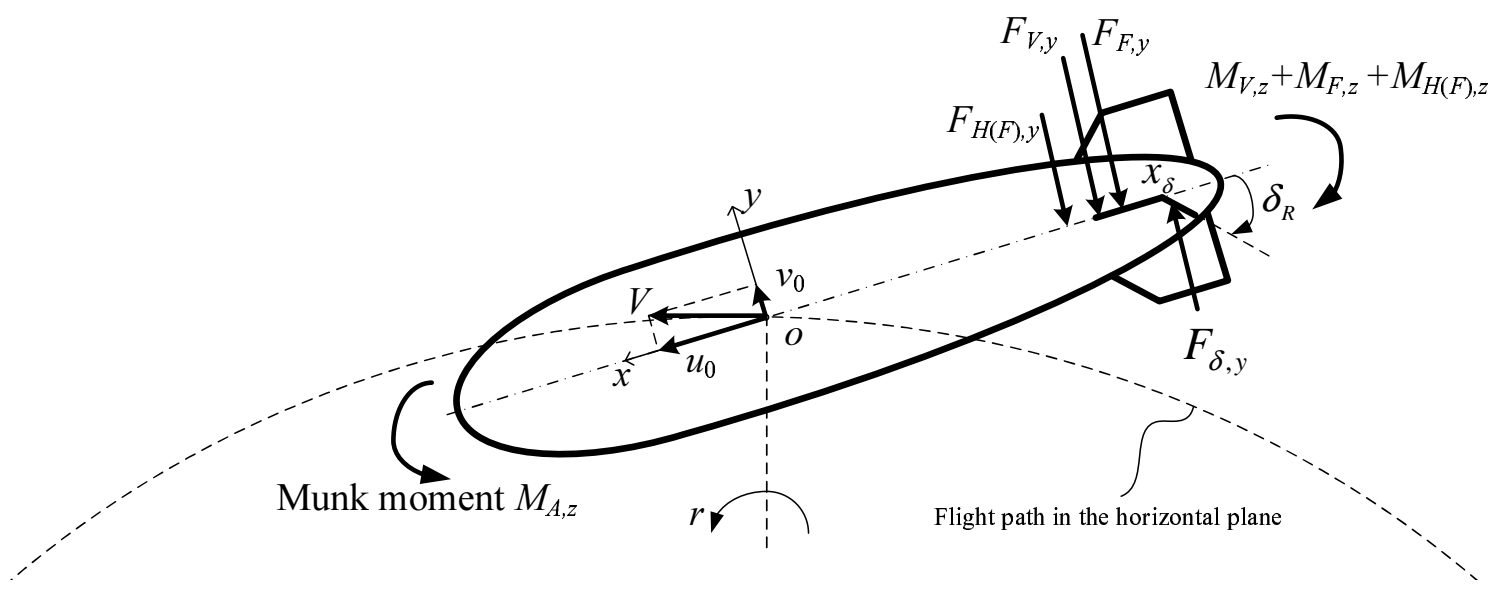

Figure 2.28: An airship in steady turn

$\delta$ denote force or moment from the aerodynamics and rudder deflection respectively. Equation (2.39) is a simplified dynamics model for a steady turn and the turn characteristics can be obtained by solving for $r$ and $v_{0}$, given the particular airspeed. The control input $F_{\delta, y}$ and $M_{\delta, z}$ can be calculated from Section 2.3.6 given a particular rudder deflection. We are now interested in evaluating the possible approximations that can be made in the computation of the aerodynamic force and moment, $F_{A D, y}$ and $M_{A D, z}$. Two computational methods are respectively applied to these terms. The first one (denoted as the full aerodynamics model) uses the approach in Sections 2.3.1 to 2.3.4 with all aerodynamic effects incorporated. The second method (denoted as the linear aerodynamics model) uses the linear formulation

$$
F_{A D, y}=\frac{\partial F_{A D, y}}{\partial v_{0}} v_{0}+\frac{\partial F_{A D, y}}{\partial r} r, \quad M_{A D, z}=\frac{\partial M_{A D, z}}{\partial v_{0}} v_{0}+\frac{\partial M_{A D, z}}{\partial r} r
$$

where the derivatives are constant and evaluated at $v_{0}=0$ and $r=0$ for the given $u_{0}$.

\subsubsection{Comparison to the 6-DOF model}

The steady turn characteristics of the Skyship-500 are now investigated using the simplified 2-DOF dynamics model with the two aerodynamics computation methods, 
and compared to the simulated results from the 6-DOF dynamics model. Figure 2.29 shows the resulting yaw rates from the different models. The 2-DOF dynamics model with linear aerodynamics computation leads to large errors, because Eq. (2.40) cannot represent the real aerodynamic effects in a turn. However, the 2-DOF model with full aerodynamics computation can accurately predict the steady turn rate, especially at low rudder deflections. The results from the 2-DOF model are slightly different from the 6-DOF dynamics model at high rudder deflections $\left(\delta_{R}>20^{\circ}\right)$. This is likely due to the effects of roll rotation.

The comparisons show that steady turn characteristics from the 2-DOF model with full aerodynamics computation are close to those from the 6-DOF nonlinear dynamics simulation; while simplifications made in the linear aerodynamics computation in Eq. (2.40) cause large error. Furthermore, the implementation of the simplified 2DOF dynamics model in Eq. (2.39) requires only the dimensional information about the airship and the $x$ coordinate of the C.G. It does not require the $z$ coordinate of the C.G. nor the second moment of inertia. This provides an accurate, effective and easy means to evaluate the turn performance of an airship. 


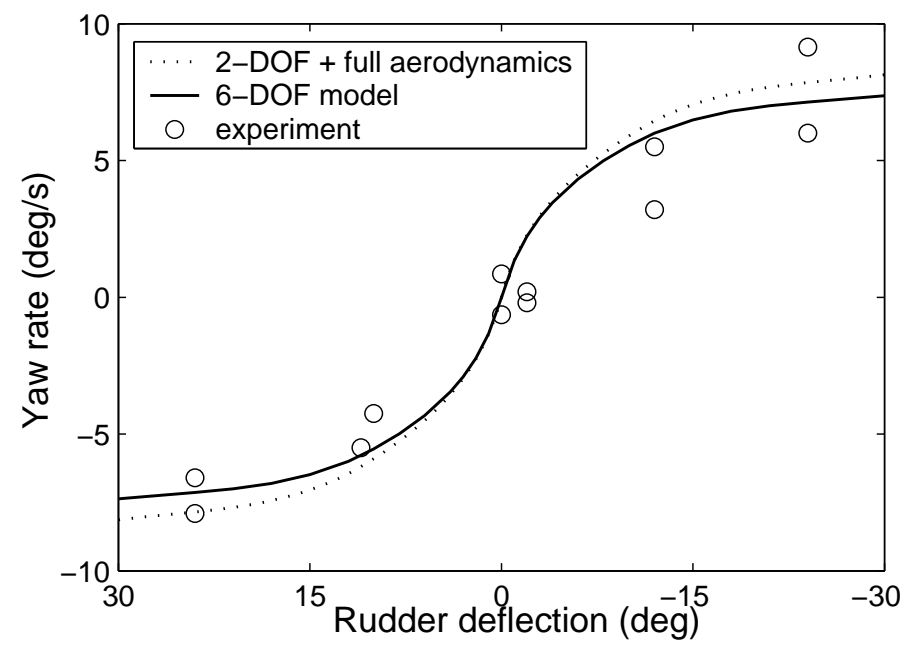

(a) 2-DOF model with full aerodynamics

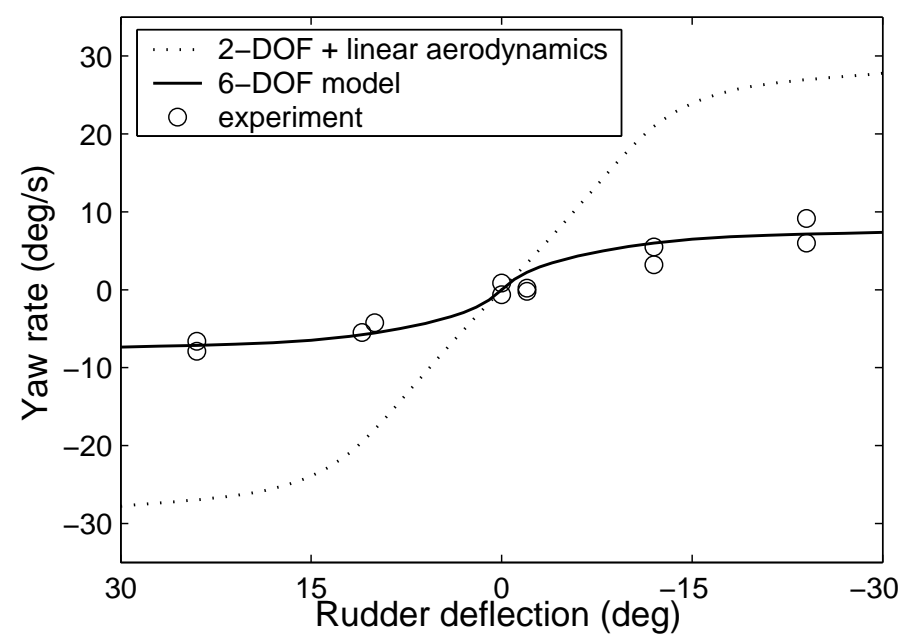

(b) 2-DOF model with linear aerodynamics

Figure 2.29: Turn rate results (at $12.86 \mathrm{~m} / \mathrm{s}$ ) from 2-DOF and 6-DOF models 


\section{Chapter 3}

\section{Dynamics Model of Flexible}

\section{Airships: Equations of Motion}

In the existing airship dynamics models, such as those presented in $[12,15,17,19]$, the aircraft was modeled as a rigid body and the structural flexibility was ignored. However, real airships experience deformations and their structural flexibility can influence the aerodynamic forces and moments and the resulting flight behavior. To improve the understanding of the airship dynamics, a theoretical framework is now presented to seamlessly unify its flight dynamics, structural dynamics, aerostatics and aerodynamics, and then the theoretical model is applied to perform the dynamics simulation and aeroelastic stability analysis for an airship. The presented dynamics model and the corresponding simulation program provide a useful tool to analyze the flight behavior and can serve as a platform to evaluate a control system design.

This chapter first assembles the equations of motion for a flexible airship with linear elastic deformation. The corresponding aerodynamics model is investigated in detail in Chapter 4, by extending the aerodynamic computational approach proposed in Chapter 2. The dynamics simulation results for a flexible airship are then presented in Chapter 5, and the possibility of aeroelastic instability is discussed in Chapter 6 . 


\subsection{Kinematic Description}

We first review the fundamental kinematic description for an unconstrained flexible vehicle. Similar to the dynamics model derived in Chapter 2, the equations of motion of an elastic aircraft are also usually derived in a body-fixed frame. As discussed by Meirovitch and Tuzcu [93], there are two commonly used body reference frames. The first one is fixed on the undeformed body; while the second one is moving relative to the undeformed body, by setting its reference axes as the mean axes. The mean axes represent a body frame satisfying six constraints such that the kinetic energy associated with the deformation stays at a minimum [112]; as a result, the linear and angular momentum vectors due to elastic deformation vanish. That is, a proper use of the mean axes for flexible bodies in vacuum can lead to inertial decoupling. However, Meirovitch et al. [93] demonstrated that, the benefits of using the mean axes are questionable for a flexible aircraft, because the equations of motion remain coupled through the aerodynamic terms. Hence, Meirovitch et al. used the first kind of the body frame for the dynamics model of a flexible airplane.

Following the lead in [93], we write the equations of motion of a flexible airship in a body frame $\{o x y z\}$ fixed on the undeformed airship (as shown in Fig. 3.1), similar to Chapter 2, with its origin $o$ at the C.V., the $x$ axis along the hull centerline and pointing toward the nose, the $z$ axis vertically downward and the positive $y$ axis determined by the right hand rule. The motion of the vehicle is described as the translation and rotation of this body frame with respect to the inertial frame $\{O X Y Z\}$, plus the deformation of the material points on the body relative to the body frame. Note that the deformed centerline (see Fig. 3.1) will be used to define local centerline frames for the aerodynamics calculation in Chapter 4.

With the aforementioned frame assignment, the elastic displacement $\mathbf{u}$ of a material point on the airship is written as a summation of the shape functions according 


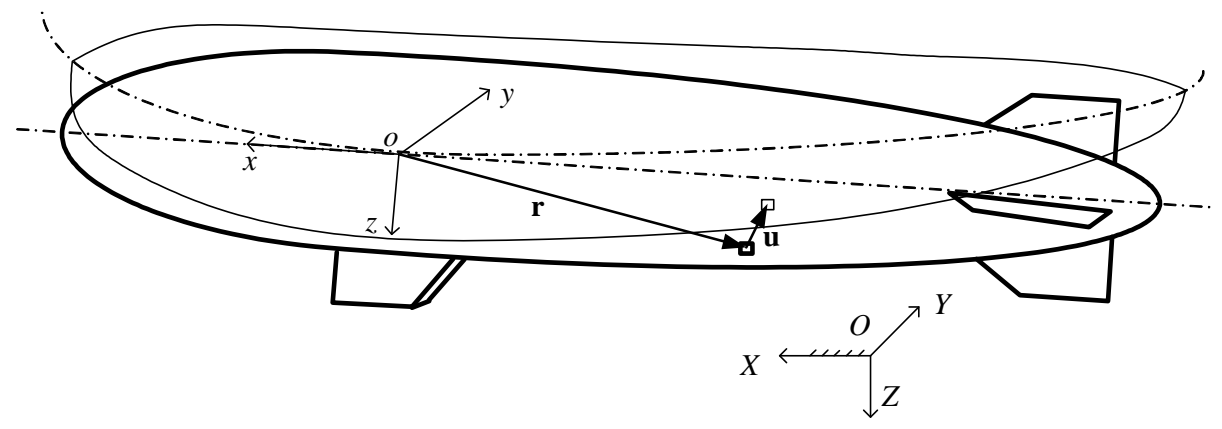

Figure 3.1: Body frame on the undeformed body

to

$$
\mathbf{u}=\sum q_{i}(t) \boldsymbol{\Phi}_{i}(\mathbf{r})
$$

where $q_{i}$ are the time-dependent generalized coordinates, $\boldsymbol{\Phi}_{i}=\left[\Phi_{x i}, \Phi_{y i}, \Phi_{z i}\right]^{\mathrm{T}}$ are the time-independent shape functions, and $\mathbf{r}$ is the position vector of the material point from the origin $o$ on the undeformed body and expressed in the body-fixed frame. Then the velocity distribution over the elastic body is expressed as:

$$
\mathbf{v}=\mathbf{v}_{0}+\boldsymbol{\omega}^{\times} \mathbf{r}+\boldsymbol{\omega}^{\times} \mathbf{u}+\dot{\mathbf{u}}
$$

where the first two terms are associated with the rigid-body translation and rotation respectively, the last two terms represent the influence of the flexibility on the local velocity. The vector $\dot{\mathbf{u}}$ is the velocity at this point due to the deformation observed in the body-fixed frame $\{$ oxyz $\}$. The term $\boldsymbol{\omega}^{\times} \mathbf{u}$ results from the coupling between rotation and deformation.

\subsubsection{Shape functions}

The shape functions can be chosen as functions that satisfy the geometric boundary conditions of the airship structure. The present document represents the first theoretical work on the aeroelastic analysis of LTA vehicles. As such, in this thesis, the airship is modeled as a free-free Euler-Bernoulli beam undergoing bending deforma- 
tion only. There are three principal reasons for using this simplified model to describe flexibility of the airship. First, from published FEA results of modal analysis of an airship with a fineness ratio of 4 [73] and an ellipsoidal shell with a fineness ratio of 6 [75], the fundamental mode shape of an airship usually describes the hull bending if the internal pressure is high enough to prevent wrinkling. Second, the deflection characteristics of non-rigid airships have been investigated using an Euler-Bernoulli beam model [2] and experiments on airship models (with fineness ratios of 4, 6.9 and 8.5) have shown that such a model could provide reasonable accuracy $[68,69]$. Third, experimental and theoretical studies have shown that Euler-Bernoulli beam models can be successfully employed to analyze the deflection behavior of inflated cylinders with fineness ratios from 6 to 18 without wrinkling [82,83,85], and that the first two vibration modes of an inflated circular cylinder are adequately described by beam bending modes [86]. A Timoshenko beam model [84] or a FEM-based method by McTavish [113] can be applied to incorporate the shear deformation and the deformation of the cross section, but would require more complicated modeling.

The shape functions in this work are chosen as the natural vibration mode shapes of the beam, because the mode shapes reflect the mass and stiffness distribution of the airship and also satisfy the orthogonality conditions; the latter can simplify the equations of motion and can be used to check the dynamics simulation program. We employ $2 N$ shape functions to describe the airship's deflection. The first $N$ shape functions are chosen as $\boldsymbol{\Phi}_{i}=\left[0, \Phi_{i}, 0\right]^{\mathrm{T}}, i=1,2, \ldots, N$, describing the bending in the oxy plane, and the other $N$ shape functions are written as $\boldsymbol{\Phi}_{N+i}=\left[0,0, \Phi_{i}\right]^{\mathrm{T}}$, $i=1,2, \ldots, N$, describing the bending in the oxz plane, where $\Phi_{i}$ is the $i^{\text {th }}$ natural mode shape of the free-free beam. That is, $\Phi_{i}$ satisfies the following spatial differential equation

$$
\frac{d^{2}}{d x^{2}}\left[E I(x) \frac{d^{2}}{d x^{2}} \Phi_{i}(x)\right]=\omega_{i}^{2} \bar{m}(x) \Phi_{i}(x)
$$


and the boundary conditions

$$
\frac{d}{d x}\left[E I(x) \frac{d^{2}}{d x^{2}} \Phi_{i}\right]=0 \quad \text { and } \quad E I(x) \frac{d^{2}}{d x^{2}} \Phi_{i}=0 \quad \text { at both ends }
$$

where $E I(x)$ is the bending stiffness, $\bar{m}$ denotes the mass per unit length and $\omega_{i}$ is the natural frequency of the $i^{\text {th }}$ mode. In addition, the normal modes of a free-free Euler-Bernoulli beam satisfy the following orthogonality conditions

$$
\int_{L} \Phi_{i} \Phi_{j} \bar{m} d x=\left\{\begin{array}{lc}
0 & \text { if } i \neq j \\
1 & \text { if } i=j
\end{array}, \quad \int_{L} \Phi_{i} \bar{m} d x=0, \quad \int_{L} x \Phi_{i} \bar{m} d x=0\right.
$$

where $L$ represents the centerline length. The first condition in Eq. (3.4) represents the orthogonality between two elastic modes, and the other two represent the orthogonality conditions between the elastic modes and rigid-body modes of a free-free beam.

\subsection{Equations of Motion}

Given the velocity distributions in Eq. (3.2), the kinetic energy of the flexible vehicle is written as

$$
\mathcal{T}=\frac{1}{2} \int_{m} \mathbf{v}^{\mathrm{T}} \mathbf{v} d m
$$

Under a linear elasticity assumption, the elastic potential energy of the flexible airship can be obtained as

$$
\mathcal{V}=\frac{1}{2} \mathbf{q}^{\mathrm{T}} \mathbf{K q}
$$

where $\mathbf{q}=\left[q_{1}, q_{2}, \ldots, q_{2 N}\right]^{\mathrm{T}}, \mathbf{K}$ is a stiffness matrix. With the shape functions from Section 3.1, the non-zero elements of the stiffness matrix $\mathbf{K}$ can be computed as

$$
K_{i, j}=\int_{L} E I(x) \Phi_{i}^{\prime \prime} \Phi_{j}^{\prime \prime} d x, \quad K_{N+i, N+j}=\int_{L} E I(x) \Phi_{i}^{\prime \prime} \Phi_{j}^{\prime \prime} d x, \quad i, j=1,2, \ldots, N
$$


The equations of motion for a flexible vehicle can be derived from the kinetic and potential energies by Lagrange's equations in terms of generalized coordinates and quasi coordinates $[93,94]$. That is

$$
\begin{array}{r}
\frac{d}{d t} \frac{\partial \mathcal{T}}{\partial \mathbf{v}_{0}}+\omega^{\times} \frac{\partial \mathcal{T}}{\partial \mathbf{v}_{0}}=\mathbf{F} \\
\frac{d}{d t} \frac{\partial \mathcal{T}}{\partial \boldsymbol{\omega}}+\mathbf{v}_{0}^{\times} \frac{\partial \mathcal{T}}{\partial \mathbf{v}_{0}}+\omega^{\times} \frac{\partial \mathcal{T}}{\partial \boldsymbol{\omega}}=\mathbf{M} \\
\frac{d}{d t} \frac{\partial \mathcal{T}}{\partial \dot{\mathbf{q}}}-\frac{\partial \mathcal{T}}{\partial \mathbf{q}}+\frac{\partial \mathcal{V}}{\partial \mathbf{q}}=\mathbf{Q}
\end{array}
$$

where $\mathbf{F}$ and $\mathbf{M}$ are the external force and moment respectively, $\mathbf{Q}$ is the generalized elastic force with its components computed as $Q_{i}=\int_{L} \mathbf{f}^{\mathrm{T}} \mathbf{\Phi}_{i} d x, i=1,2, \ldots, 2 N$, in which $\mathbf{f}$ is the external force per unit length. The resulting equations of motion have the following matrix form:

$$
\left[\begin{array}{lll}
\mathbf{M}_{11} & \mathbf{M}_{12} & \mathbf{M}_{13} \\
\mathbf{M}_{21} & \mathbf{M}_{22} & \mathbf{M}_{23} \\
\mathbf{M}_{31} & \mathbf{M}_{32} & \mathbf{M}_{33}
\end{array}\right]\left[\begin{array}{c}
\dot{\mathbf{v}}_{0} \\
\dot{\boldsymbol{\omega}} \\
\ddot{\mathbf{q}}
\end{array}\right]=-\left[\begin{array}{c}
\mathbf{0} \\
\mathbf{0} \\
\mathbf{S}_{E}
\end{array}\right]+\left[\begin{array}{c}
\mathbf{F}_{I} \\
\mathbf{M}_{I} \\
\mathbf{Q}_{I}
\end{array}\right]+\left[\begin{array}{c}
\mathbf{F}_{G} \\
\mathbf{M}_{G} \\
\mathbf{Q}_{G}
\end{array}\right]+\left[\begin{array}{c}
\mathbf{F}_{A S} \\
\mathbf{M}_{A S} \\
\mathbf{Q}_{A S}
\end{array}\right]+\left[\begin{array}{c}
\mathbf{F}_{A D} \\
\mathbf{M}_{A D} \\
\mathbf{Q}_{A D}
\end{array}\right]+\left[\begin{array}{c}
\mathbf{F}_{C} \\
\mathbf{M}_{C} \\
\mathbf{Q}_{C}
\end{array}\right]
$$

where

$$
\mathbf{M}_{\text {sys }}=\left[\begin{array}{lll}
\mathbf{M}_{11} & \mathbf{M}_{12} & \mathbf{M}_{13} \\
\mathbf{M}_{21} & \mathbf{M}_{22} & \mathbf{M}_{23} \\
\mathbf{M}_{31} & \mathbf{M}_{32} & \mathbf{M}_{33}
\end{array}\right]
$$

is the symmetric mass matrix of the flexible airship, $\mathbf{S}_{E}$ is the internal elastic force vector and represents the stiffness term as

$$
\mathbf{S}_{E}=\mathbf{K q}
$$

The subscripts $I, G, A S, A D$ and $C$ denote the forces and moments due to inertia, gravity, aerostatics, aerodynamics, and control, respectively. The computation of the forces, moments and generalized forces due to aerodynamics will be investigated in 
detail in Chapter 4. The other force and moment terms are discussed below.

\subsection{Inertial Terms}

\subsubsection{Linear and angular momenta}

In order to introduce the definitions of the shape integrals used in the mass matrix, and to interpret the corresponding integral results to be presented in Section 5.3.2.1, we proceed to write the linear and angular momenta for a flexible vehicle.

The linear momentum of the airship is defined as $\int_{m} \mathbf{v} d m$. Substituting $\mathbf{v}$ from Eq. (3.2) yields

$$
\int_{m} \mathbf{v} d m=m \mathbf{v}_{0}+\boldsymbol{\omega}^{\times} \mathbf{r}_{G}+\boldsymbol{\omega}^{\times} \sum_{i=1}^{2 N} q_{i} \mathbf{p}_{i}+\sum_{i=1}^{2 N} \dot{q}_{i} \mathbf{p}_{i}
$$

where $\mathbf{r}_{G}=\int_{m} \mathbf{r} d m / m$ is the position vector of the C.G. of the undeformed body, and the linear momentum coefficients

$$
\mathbf{p}_{i}=\int_{m} \mathbf{\Phi}_{i} d m, \quad i=1,2, \ldots, 2 N
$$

represent the influence of the $i^{\text {th }}$ mode shape on the position of the C.G.

The angular momentum of a flexible vehicle about the origin of the body-fixed frame $o$ is obtained as $\int_{m}(\mathbf{r}+\mathbf{u})^{\times} \mathbf{v} d m$. Substituting $\mathbf{u}$ from Eq. (3.1) and $\mathbf{v}$ from Eq. (3.2) leads to

$$
\int_{m}\left(\mathbf{r}^{\times}+\mathbf{u}^{\times}\right) \mathbf{v} d m=\mathbf{J}_{\text {total }} \boldsymbol{\omega}+m \mathbf{r}_{G}^{\times} \mathbf{v}_{0}+\sum_{i=1}^{2 N} q_{i} \mathbf{p}_{i}^{\times} \mathbf{v}_{0}+\sum_{i=1}^{2 N} \dot{q}_{i} \mathbf{h}_{i}+\sum_{i=1}^{2 N} \sum_{i=1}^{2 N} q_{i} \dot{q}_{j} \mathbf{h}_{i j}^{\prime}
$$


The matrix $\mathbf{J}_{\text {total }}$ in Eq. (3.16) is the total second moment of inertia computed as

$$
\mathbf{J}_{\text {total }}=-\int_{m}(\mathbf{r}+\mathbf{u})^{\times}(\mathbf{r}+\mathbf{u})^{\times} d m=\mathbf{J}+\mathbf{J}_{r u}+\mathbf{J}_{u r}+\mathbf{J}_{u u}
$$

where $\mathbf{J}=-\int_{m} \mathbf{r}^{\times} \mathbf{r}^{\times} d m$ is the second moment of inertia of the undeformed vehicle. The other three terms denote the effect of the elastic displacement on the second moment of inertia. The matrices $\mathbf{J}_{r u}$ and $\mathbf{J}_{u r}$ are the rigid/flexible coupling parts of the second moment of inertia and $\mathbf{J}_{u r}=\mathbf{J}_{r u}^{\mathrm{T}}$, in which $\mathbf{J}_{r u}$ is obtained as

$$
\mathbf{J}_{r u}=-\int_{m} \mathbf{r}^{\times} \mathbf{u}^{\times} d m=\sum_{i=1}^{2 N} q_{i} \mathbf{J}_{r u, i}^{\prime} \quad \text { where } \quad \mathbf{J}_{r u, i}^{\prime}=-\int_{m} \mathbf{r}^{\times} \boldsymbol{\Phi}_{i}^{\times} d m
$$

The matrix $\mathbf{J}_{u u}$ is the flexible part of the second moment of inertia determined with

$$
\mathbf{J}_{u u}=-\int_{m} \mathbf{u}^{\times} \mathbf{u}^{\times} d m=\sum_{i=1}^{2 N} \sum_{j=1}^{2 N} q_{i} q_{j} \mathbf{J}_{u u, i j}^{\prime \prime} \quad \text { where } \quad \mathbf{J}_{u u, i j}^{\prime \prime}=-\int_{m} \boldsymbol{\Phi}_{i}^{\times} \boldsymbol{\Phi}_{j}^{\times} d m
$$

The third term in Eq. (3.16) represents the influence of the elastic displacement of the C.G. on the angular momentum. The angular momentum coefficients and the flexible angular momentum coefficients are defined as

$$
\mathbf{h}_{i}=\int_{m} \mathbf{r}^{\times} \boldsymbol{\Phi}_{i} d m, \quad \mathbf{h}_{i j}^{\prime}=\int_{m} \boldsymbol{\Phi}_{i}^{\times} \boldsymbol{\Phi}_{j} d m, \quad i, j=1,2, \ldots, 2 N
$$

The fourth and fifth terms in Eq. (3.16) denote the effects of the generalized velocities on the angular momentum.

\subsubsection{Mass matrix and inertial force}

The expressions for the symmetric mass matrix $\mathbf{M}_{\text {sys }}$ and the inertial terms $\mathbf{F}_{I}, \mathbf{M}_{I}$ and $\mathbf{Q}_{I}$ of an elastic body have been derived for robotic manipulators [114] and conventional aircraft [113] and are no different for the elastic airships. Therefore we do 
not repeat their derivations but only summarize the results in this section.

The submatrices in the mass matrix in Eq. (3.12) can be written as $[113,114]$

$$
\begin{array}{lll}
\mathbf{M}_{11}=m \mathbf{I}_{3 \times 3}, & \mathbf{M}_{12}=-\mathbf{c}_{\text {total }}^{\times}, & \mathbf{M}_{13}=\mathbf{P} \\
\mathbf{M}_{21}=\mathbf{c}_{\text {total }}^{\times}, & \mathbf{M}_{22}=\mathbf{J}_{\text {total }}, & \mathbf{M}_{23}=\mathbf{H}_{\text {total }} \\
\mathbf{M}_{31}=\mathbf{P}^{\mathrm{T}}, & \mathbf{M}_{32}=\mathbf{H}_{\text {total }}^{\mathrm{T}}, & \mathbf{M}_{33}=\mathbf{M}_{e}
\end{array}
$$

The $3 \times 2 N$ linear momentum coefficient matrix $\mathbf{P}$ is associated with the influence of deformation on the linear momentum and is defined as:

$$
\mathbf{P}=\left[\mathbf{p}_{1}, \mathbf{p}_{2}, \ldots, \mathbf{p}_{2 N}\right]
$$

The total first moment of inertia $\mathbf{c}_{\text {total }}$ is obtained as

$$
\mathbf{c}_{\text {total }}=\int_{m}(\mathbf{r}+\mathbf{u}) d m=m \mathbf{r}_{G}+\mathbf{P q}
$$

where the $\mathbf{P q}$ term represents the change of the first moment because of elastic deformation. In general, $\mathbf{P q}$ is not zero, in which case the translation and rotation remain coupled for a flexible vehicle even if the body frame is established at the C.G. of the undeformed body. This also shows that the advantages of using a body-fixed frame at the C.G. is questionable for an elastic airship.

The $3 \times 2 N$ matrix $\mathbf{H}_{\text {total }}$ represents the influence of elastic deformation on the angular momentum of a flexible vehicle and it consists of two components, i.e.,

$$
\mathbf{H}_{\text {total }}=\mathbf{H}+\mathbf{H}_{u}
$$

in which $\mathbf{H}$ is the angular momentum coefficient matrix defined as

$$
\mathbf{H}=\left[\mathbf{h}_{1}, \mathbf{h}_{2}, \ldots, \mathbf{h}_{2 N}\right]
$$


and the flexible angular momentum coefficient matrix $\mathbf{H}_{u}$ is given as

$$
\mathbf{H}_{u}=\sum_{i=1}^{2 N} q_{i} \mathbf{H}_{u, i}^{\prime} \quad \text { where } \quad \mathbf{H}_{u, i}^{\prime}=\left[\mathbf{h}_{i 1}^{\prime}, \mathbf{h}_{i 2}^{\prime}, \ldots, \mathbf{h}_{i, 2 N}^{\prime}\right]
$$

Lastly, the components of the elastic generalized mass matrix $\mathbf{M}_{e}$ are defined as

$$
M_{e, i j}=\int_{m} \boldsymbol{\Phi}_{i}^{\mathrm{T}} \boldsymbol{\Phi}_{j} d m
$$

From the first orthogonality condition in Eq. (3.4), the elastic generalized mass matrix $\mathbf{M}_{e}$ is a $2 N \times 2 N$ identity matrix. This completes the formulation of the mass matrix of a flexible airship. In summary, the submatrices $\mathbf{M}_{11}, \mathbf{M}_{13}, \mathbf{M}_{31}$ and $\mathbf{M}_{33}$ are constant, while other submatrices depend on the generalized coordinates. Consequently, the total mass matrix is an implicit function of time.

The inertial force and moment terms on the right hand side of Eq. (3.11) are given by $[113,114]$ :

$$
\left[\begin{array}{c}
\mathbf{F}_{I} \\
\mathbf{M}_{I} \\
\mathbf{Q}_{I}
\end{array}\right]=-\left[\begin{array}{ccc}
m \boldsymbol{\omega}^{\times} & -\boldsymbol{\omega}^{\times} \mathbf{c}_{\text {total }}^{\times} & 2 \mathbf{P}_{\dot{q}} \\
\mathbf{c}_{\text {total }}^{\times} \boldsymbol{\omega}^{\times} & \boldsymbol{\omega}^{\times} \mathbf{J}_{\text {total }} & 2 \mathbf{H}_{\dot{q}, \text { total }} \\
-\mathbf{P}_{\dot{q}}^{\mathrm{T}} & -\mathbf{H}_{\dot{q}, \text { total }}^{\mathrm{T}} & 2 \mathbf{M}_{e \dot{q}}
\end{array}\right]\left[\begin{array}{c}
\mathbf{v}_{0} \\
\boldsymbol{\omega} \\
\dot{\mathbf{q}}
\end{array}\right]
$$

in which the effects of elastic deformation on the centrifugal terms are included in the total first moment of inertia $\mathbf{c}_{\text {total }}$. Again, the centrifugal force and moment are not zero even if the body frame is at the C.G. of the undeformed vehicle. The effects of flexibility on the Coriolis terms are accounted for by the matrices $\mathbf{P}_{\dot{q}}, \mathbf{H}_{\dot{q}, \text { total }}$ and $\mathbf{M}_{e \dot{q}}$. The definitions of these matrices are given as follows.

The $3 \times 2 N$ flexible linear Coriolis matrix is $\mathbf{P}_{\dot{q}}=\boldsymbol{\omega}^{\times} \mathbf{P}$ and the force term $-2 \mathbf{P}_{\dot{q}} \dot{\mathbf{q}}$ represents the Coriolis force because of the deformation. The $3 \times 2 N$ flexible angular 
Coriolis matrix $\mathbf{H}_{\dot{q}, \text { total }}$ is computed as $\mathbf{H}_{\dot{q}, \text { total }}=\mathbf{H}_{\dot{q}}+\mathbf{H}_{\dot{q} u}$, in which

$$
\mathbf{H}_{\dot{q}}=\left[\mathbf{J}_{r u, 1}^{\prime} \boldsymbol{\omega}, \mathbf{J}_{r u, 2}^{\prime} \boldsymbol{\omega}, \ldots, \mathbf{J}_{r u, 2 N}^{\prime} \boldsymbol{\omega}\right]
$$

and

$$
\mathbf{H}_{\dot{q} u}=\left[\sum_{j=1}^{2 N} q_{j} \mathbf{J}_{u u, 1 j}^{\prime \prime} \boldsymbol{\omega}, \sum_{j=1}^{2 N} q_{j} \mathbf{J}_{u u, 2 j}^{\prime \prime} \boldsymbol{\omega}, \ldots, \sum_{j=1}^{2 N} q_{j} \mathbf{J}_{u u, 2 N j}^{\prime \prime} \boldsymbol{\omega}\right]
$$

The $2 N \times 2 N$ flexible Coriolis matrix is defined as

$$
\mathbf{M}_{e \dot{q}}=\left[\boldsymbol{\omega}^{\times} \mathbf{H}_{u, 1}^{\prime}, \boldsymbol{\omega}^{\times} \mathbf{H}_{u, 2}^{\prime}, \ldots, \boldsymbol{\omega}^{\times} \mathbf{H}_{u, 2 N}^{\prime}\right]
$$

We can see that the centrifugal and Coriolis terms are nonlinear functions of the rigid-body velocities $\left(\mathbf{v}_{0}\right.$ and $\left.\boldsymbol{\omega}\right)$ and the elastic deformation ( $\mathbf{q}$ and $\left.\dot{\mathbf{q}}\right)$.

Equations (3.21) to (3.23), and Eq. (3.30) provide the complete formulations of the total mass matrix and the inertial forces and moments for an unconstrained flexible vehicle. These were derived from the exact kinetic energy expression, i.e., Eq. (3.5) with the exact velocity distribution of Eq. (3.2) and therefore they include all inertial elastic nonlinearities.

\subsection{Gravity, Aerostatic and Thrust Forces}

The airship flexibility does not affect the magnitude of the gravitational force, so that

the force vector $\mathbf{F}_{G}$ can be calculated using the method for a rigid-body model, i.e., with Eq. (2.5). However, the gravitational moment is affected by the deformation because of the elastic displacement of the C.G., which is computed as $\mathbf{u}_{G}=\int_{m} \mathbf{u} d m / m$. Substituting Eq. (3.1) for $\mathbf{u}$ leads to

$$
\mathbf{u}_{G}=\frac{1}{m} \sum_{i=1}^{2 N} q_{i}(t) \mathbf{p}_{i}
$$


The external moment due to gravity is then computed as

$$
\mathbf{M}_{G}=\mathbf{r}_{G}^{\times} \mathbf{F}_{G}+\frac{1}{m} \sum_{i=1}^{2 N} q_{i}(t) \mathbf{p}_{i}^{\times} \mathbf{F}_{G}
$$

The second term on the right hand side of Eq. (3.35) represents the influence of flexibility on the gravitational moment. The gravitational generalized force is obtained as $Q_{G i}=\int_{m} g \hat{\mathbf{g}}^{\mathrm{T}} \boldsymbol{\Phi}_{i} d m$ or using the linear momentum coefficients of Eq. (3.15), we have

$$
Q_{G i}=g \hat{\mathbf{g}}^{\mathrm{T}} \mathbf{p}_{i}, \quad i=1,2, \ldots, 2 N
$$

The aerostatic force $F_{A S}$ can be computed using Eq. (2.7), since it is reasonable to assume that the flexibility does not affect the body volume. The elastic displacement of the C.V. is computed as $\mathbf{u}_{V}=\int_{V} \mathbf{u} d V / V_{B}$. Then, substituting Eq. (3.1) to determine $\mathbf{u}_{V}$, the external moment due to aerostatics can be written as

$$
\mathbf{M}_{A S}=\sum_{i=1}^{2 N} q_{i} \mathbf{I}_{A S, i}^{\times} \mathbf{F}_{A S}
$$

where we define an aerostatic shape function integral $\mathbf{I}_{A S, i}$ for the $i^{\text {th }}$ mode as

$$
\mathbf{I}_{A S, i}=\frac{1}{V_{B}} \int_{V} \boldsymbol{\Phi}_{i} d V=\frac{1}{V_{B}} \int_{L} \boldsymbol{\Phi}_{i} S(x) d x
$$

The aerostatic generalized force is computed as $Q_{A S, i}=-\int_{L} \rho \hat{\mathbf{g}}^{\mathrm{T}} \mathbf{\Phi}_{i} S d x$, or, using the shape function of Eq. (3.38), we have:

$$
Q_{A S, i}=\mathbf{F}_{A S}^{\mathrm{T}} \mathbf{I}_{A S, i}, \quad i=1,2, \ldots, 2 N
$$

The aerodynamically-induced control forces due to flap deflection will be investigated in Chapter 4, but the thrust forces are discussed in this section. Unlike the distributed gravity and aerostatic forces, the thrust forces are concentrated forces. 
The corresponding external force $\mathbf{F}_{T}$ can be computed using the method described in Section 2.5.2.1. The thruster moment is obtained as $\mathbf{M}_{T}=\mathbf{r}_{T}^{\times} \mathbf{F}_{T}+\mathbf{u}_{T}^{\times} \mathbf{F}_{T}$ in which $\mathbf{u}_{T}$ denotes the elastic displacement at the position $\mathbf{r}_{T}$ where a thruster is mounted. Again, using Eq. (3.1), we obtain:

$$
\mathbf{M}_{T}=\mathbf{r}_{T}^{\times} \mathbf{F}_{T}+\sum_{i=1}^{2 N} q_{i}(t) \boldsymbol{\Phi}_{i}\left(\mathbf{r}_{T}\right)^{\times} \mathbf{F}_{T}
$$

in which $\mathbf{\Phi}_{i}\left(\mathbf{r}_{T}\right)$ denotes the shape function evaluated at the thruster position. The corresponding generalized force is given as

$$
Q_{T i}=\mathbf{F}_{T}^{\mathrm{T}} \boldsymbol{\Phi}_{i}\left(\mathbf{r}_{T}\right)
$$

We can see that the influence of flexibility on the thruster model is incorporated in the moment calculation by accounting for the elastic displacement at the thruster position. 


\section{Chapter 4}

\section{Dynamics Model of Flexible Airships: Aerodynamics}

To complete the dynamics model presented in Chapter 3, the aerodynamic force, moment and generalized force should be incorporated into Eq. (3.11). For this purpose, we now extend the aerodynamic computational method in Chapter 2 to flexible airships. With the aerodynamic terms categorized in the same way as in Section 2.3, the aerodynamic formulation can be summarized as follows

$$
\left[\begin{array}{c}
\mathbf{F}_{A D} \\
\mathbf{M}_{A D} \\
\mathbf{Q}_{A D}
\end{array}\right]=\left[\begin{array}{c}
\mathbf{F}_{A} \\
\mathbf{M}_{A} \\
\mathbf{Q}_{A}
\end{array}\right]+\left[\begin{array}{c}
\mathbf{F}_{V} \\
\mathbf{M}_{V} \\
\mathbf{Q}_{V}
\end{array}\right]+\left[\begin{array}{c}
\mathbf{F}_{F} \\
\mathbf{M}_{F} \\
\mathbf{Q}_{F}
\end{array}\right]+\left[\begin{array}{c}
\mathbf{F}_{H(F)} \\
\mathbf{M}_{H(F)} \\
\mathbf{Q}_{H(F)}
\end{array}\right]+\left[\begin{array}{c}
\mathbf{F}_{\text {Axial }} \\
\mathbf{M}_{\text {Axial }} \\
\mathbf{Q}_{\text {Axial }}
\end{array}\right]
$$

where the first term is the potential-flow aerodynamic force, which is related to the added mass, the second term is due to the viscous effect on the hull, the third term is the force acting on the fins, the fourth term is the force on the hull due to the fins, and the last term is associated with the axial drag. The computation of each term will be discussed in the following sections but first we revisit the velocity distribution of a flexible airship employed in these computations. 


\subsection{Velocity Computation for Aerodynamics}

To consider the effects of flexibility on the aerodynamic computation, the local velocity

components defined in a local centerline frame $\left\{P x_{p} y_{p} z_{p}\right\}$ (shown in Fig. 4.1) are used in the aerodynamics computation for a flexible airship. This centerline frame is established along the centerline at a point $P$, with its $x_{p}$ axis tangent to the centerline. For a rigid airship, the $x_{p}$ axis is parallel to the $x$ axis of the body-fixed frame $\{o x y z\}$; for a flexible airship, the orientation of the frame $\left\{P x_{p} y_{p} z_{p}\right\}$ is related to the bending slopes. For small elastic displacements and infinitesimal rotations, we use the firstorder approximation to compute the rotation matrix from the body frame to the local centerline frame $\mathbf{R}_{p}$ [114], i.e.,

$$
\mathbf{R}_{p}=\left[\begin{array}{ccc}
1 & R_{12} & R_{13} \\
-R_{12} & 1 & 0 \\
-R_{13} & 0 & 1
\end{array}\right] \quad \text { where } \quad R_{12}=\sum_{i=1}^{2 N} q_{i} \Phi_{y i}^{\prime}, \quad R_{13}=\sum_{i=1}^{2 N} q_{i} \Phi_{z i}^{\prime}
$$

The velocity distribution of a flexible airship, i.e., $\mathbf{v}$ from Eq. (3.2), is now rewritten in the local centerline frame $\left\{P x_{p} y_{p} z_{p}\right\}$ as

$$
\mathbf{v}_{d}=\mathbf{R}_{p} \mathbf{v}
$$

where the subscript $d$ denotes a vector written in a local centerline frame on the deformed body. Substituting $\mathbf{R}_{p}$ from Eq. (4.2) and $\mathbf{v}$ from Eq. (3.2) into Eq. (4.3), and neglecting the products of the following terms of small orders of magnitude: $\left(p, q, r, \Phi_{i}, \Phi_{i}^{\prime}\right)$, we have

$$
\mathbf{v}_{d} \approx \mathbf{v}_{0}+\omega^{\times} \mathbf{r}+\sum_{i=1}^{2 N} \dot{q}_{i} \boldsymbol{\Phi}_{i}-u_{0} \sum_{i=1}^{2 N} q_{i} \boldsymbol{\Phi}_{i}^{\prime}
$$

where the first two terms represent the velocity distribution on a rigid-body vehicle; 


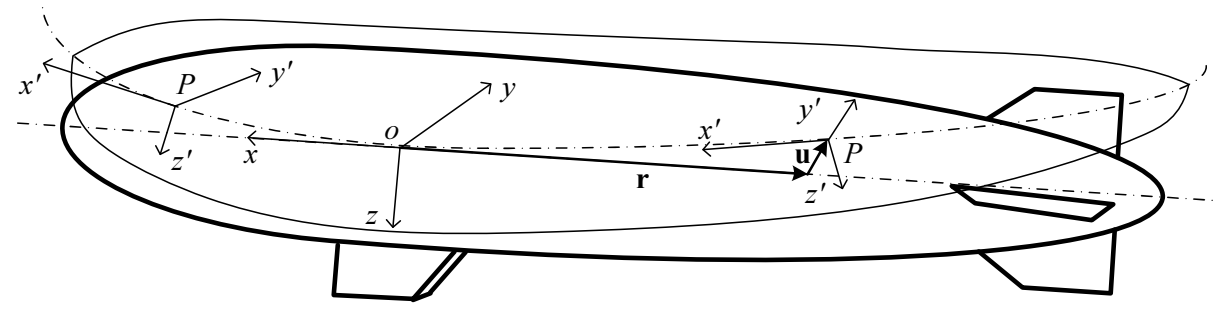

Figure 4.1: Local centerline frame

the last two terms represent the influence of deformation: one related to the deflection rate and the other related to the bending slope. To incorporate the effects of flexibility, $\mathbf{v}_{d}=\left\{u_{d}, v_{d}, w_{d}\right\}$ calculated as Eq. (4.4) is used in the aerodynamics computations in this chapter. A similar velocity calculation was used to compute the local angle of attack of an elastic missile in [96].

\subsection{Potential-flow aerodynamic force}

For a rigid-body vehicle completely submerged in an unbounded potential fluid, the fluid kinetic energy can be written in terms of a $6 \times 6$ added-mass matrix and the aerodynamic forces and moments are given by the added-mass terms in Eq. (2.9). For a flexible vehicle, with its deformation described by $2 N$ shape functions, the added-mass matrix representing the fluid kinetic energy should be a $(6+2 N) \times(6+2 N)$ matrix. To the author's knowledge, no works have been published on a complete formulation of the added-mass matrix and the corresponding aerodynamic forces and moments for an elastic vehicle maneuvering in a heavy fluid. In this subsection, the derivation of this added-mass matrix and the computation of the corresponding forces and moments are presented from first principles. For this purpose, the boundary condition of the Laplace equation is first given for an elastic vehicle moving in an unbounded potential fluid, then the total added-mass matrix and the corresponding aerodynamic forces, moments and generalized forces are derived from the fluid's kinetic energy. Finally, the airship is approximated as an ellipsoid of revolution and the added-mass matrix 
is evaluated using the analytical solution of the Laplace equation for an ellipsoid.

\subsubsection{Boundary condition of the Laplace equation}

For a potential fluid, the velocity vector of the fluid can be represented by the gradient of a scalar potential $\Psi[25]$, i.e., $\nabla \Psi=\mathbf{v}_{f}$, where $\mathbf{v}_{f}=\left[u_{f}, v_{f}, w_{f}\right]^{\mathrm{T}}$ denotes the velocity vector of the fluid. The continuity equation for the fluid can be written as the Laplace equation [25]

$$
\nabla^{2} \Psi=0
$$

In the case of a vehicle in an unbounded fluid, no fluid particles can flow through the boundary surface into the body, so at a point on the boundary surface, the normal component of the fluid velocity must be equal to the normal velocity of that point on the body, as shown in Fig. 4.2, i.e.,

$$
\mathbf{v}_{f}^{\mathrm{T}} \mathbf{n}=\mathbf{v}_{d}^{\mathrm{T}} \mathbf{n}, \quad \text { over } \quad S_{B}
$$

where $\mathbf{n}=\left[n_{1}, n_{2}, n_{3}\right]^{\mathrm{T}}$ is the unit normal vector of the boundary surface, with its positive direction defined outside the body and $S_{B}$ denotes the boundary surface of the body. Substituting $\mathbf{v}_{d}$ from Eq. (4.4) into Eq. (4.6), we have

$$
\mathbf{v}_{f}^{\mathrm{T}} \mathbf{n}=\mathbf{v}_{0}^{\mathrm{T}} \mathbf{n}+\boldsymbol{\omega}^{\mathrm{T}}\left(\mathbf{r}^{\times} \mathbf{n}\right)+\sum_{i=1}^{2 N} \dot{q}_{i} \boldsymbol{\Phi}_{i}^{\mathrm{T}} \mathbf{n}-u_{0} \sum_{i=1}^{2 N} q_{i} \boldsymbol{\Phi}_{i}^{\prime \mathrm{T}} \mathbf{n}, \quad \text { over } \quad S_{B}
$$

Equation (4.7) represents the boundary conditions of the Laplace equation for a flexible body moving through an unbounded potential fluid.

In order to separate the variables according to the boundary conditions, we write the velocity potential as a superposition of various velocity potential components as

$$
\Psi=\boldsymbol{v}^{\mathrm{T}} \boldsymbol{\Psi}_{r}+\dot{\mathbf{q}}^{\mathrm{T}} \boldsymbol{\Psi}_{q}+u_{0} \mathbf{q}^{\mathrm{T}} \boldsymbol{\Psi}_{s}
$$




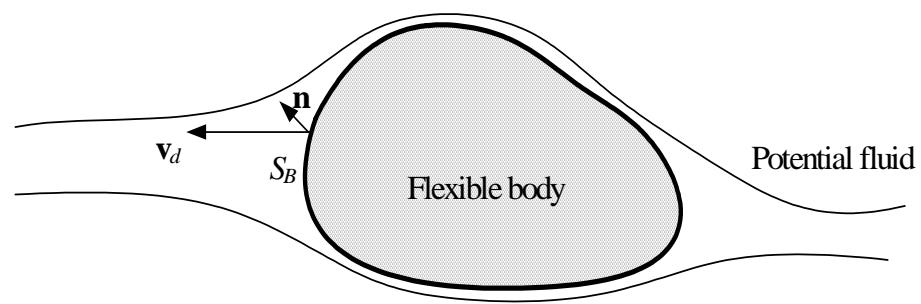

Figure 4.2: Boundary condition of the Laplace equation

Recall that $\boldsymbol{v}=\left[\mathbf{v}_{0}^{\mathrm{T}}, \boldsymbol{\omega}^{\mathrm{T}}\right]^{\mathrm{T}}$ and $\mathbf{v}_{0}=\left[u_{0}, v_{0}, w_{0}\right]^{\mathrm{T}}, \boldsymbol{\omega}=[p, q, r]^{\mathrm{T}}$ denote the linear and angular velocity vectors. The vector $\Psi_{r}=\left[\Psi_{r 1}, \Psi_{r 2}, \ldots, \Psi_{r 6}\right]^{\mathrm{T}}$ represents the velocity potentials associated with the rigid-body motion, in which $\Psi_{r 1}=\left[\Psi_{r 1}, \Psi_{r 2}, \Psi_{r 3}\right]^{\mathrm{T}}$ contains the potential components related to the translational motion and $\Psi_{r 2}=$ $\left[\Psi_{r 4}, \Psi_{r 5}, \Psi_{r 6}\right]^{\mathrm{T}}$ contains the components related to the rotational motion. The potentials $\Psi_{r i}$ are functions of position only and satisfy the Laplace equation $\nabla^{2} \Psi_{r i}=0$ $(i=1,2, \ldots, 6)$. The components related to translation and rotation satisfy the following boundary conditions respectively,

$$
\frac{\partial \boldsymbol{\Psi}_{r 1}}{\partial n}=\mathbf{n}, \quad \frac{\partial \boldsymbol{\Psi}_{r 2}}{\partial n}=\mathbf{r}^{\times} \mathbf{n}
$$

where $\partial \Psi_{r 1} / \partial n=\left[\partial \Psi_{r 1} / \partial n, \ldots, \partial \Psi_{r 3} / \partial n\right]^{\mathrm{T}}, \partial \Psi_{r 2} / \partial n=\left[\partial \Psi_{r 4} / \partial n, \ldots, \partial \Psi_{r 6} / \partial n\right]^{\mathrm{T}}$, and $\partial \Psi_{r 1} / \partial n \equiv\left(\nabla \Psi_{r 1}\right)^{\mathrm{T}} \mathbf{n}$, etc.

The second term on the right hand side of Eq. (4.8) is associated with the deflection rate. The vector $\Psi_{q}=\left[\Psi_{q 1}, \Psi_{q 2}, \ldots, \Psi_{q, 2 N}\right]^{\mathrm{T}}$ contains the potential components related to the mode shapes. That is, $\Psi_{q i}$ is independent of time and satisfies the Laplace equation with the corresponding boundary condition as

$$
\nabla^{2} \Psi_{q i}=0, \quad \frac{\partial \Psi_{q i}}{\partial n}=\boldsymbol{\Phi}_{i}^{\mathrm{T}} \mathbf{n}, \quad i=1,2, \ldots, 2 N
$$

The third term on the right hand side of Eq. (4.8) relates to the bending slope. The vector $\Psi_{s}=\left[\Psi_{s 1}, \Psi_{s 2}, \ldots, \Psi_{s, 2 N}\right]^{\mathrm{T}}$ contains the potential components associated with the slopes of the shape functions. Similarly, $\Psi_{s i}$ is independent of time and 
satisfies the Laplace equation with the corresponding boundary condition as

$$
\nabla^{2} \Psi_{s i}=0, \quad \frac{\partial \Psi_{s i}}{\partial n}=-\boldsymbol{\Phi}_{i}^{\prime \mathrm{T}} \mathbf{n}, \quad i=1,2, \ldots, 2 N
$$

Under the conditions of Eqs. (4.9) to (4.11), we can check that the velocity potential in Eq. (4.8) is a solution of the Laplace equation with the boundary conditions of a flexible vehicle in Eq. (4.7). Once all the components $\Psi_{r i}(i=1,2, \ldots, 6), \Psi_{q i}$ $(i=1,2, \ldots, 2 N)$ and $\Psi_{s i}(i=1,2, \ldots, 2 N)$ are known, we can solve the Laplace equation for $\Psi$.

\subsubsection{Fluid kinetic energy and added-mass matrix}

The fluid kinetic energy of a volume element $d V$ is $1 / 2 \rho\left(\mathbf{v}_{f}^{\mathrm{T}} \mathbf{v}_{f}\right) d V$. Expressing the velocity in terms of the velocity potential and integrating over the region of the fluid, we have the total kinetic energy of the fluid as $\mathcal{T}_{f}=\frac{1}{2} \rho \iiint_{V_{f}}(\nabla \Psi)^{\mathrm{T}}(\nabla \Psi) d V$, in which $V_{f}$ denotes the fluid volume. Using Green's theorem, the kinetic energy of the fluid is written as

$$
\mathcal{T}_{f}=-\frac{1}{2} \rho \iint_{S_{B}} \Psi \frac{\partial \Psi}{\partial n} d S
$$

With the velocity potential written as the superposition of $\Psi_{r i}, \Psi_{q i}$ and $\Psi_{s i}$ from Eq. (4.8), we have

$$
\begin{aligned}
T_{f}= & \frac{1}{2} \boldsymbol{v}^{\mathrm{T}} \mathbf{M}_{r r} \boldsymbol{v}+\frac{1}{2} \boldsymbol{v}^{\mathrm{T}} \mathbf{M}_{r q} \dot{\mathbf{q}}+\frac{1}{2} \dot{\mathbf{q}}^{\mathrm{T}} \mathbf{M}_{q r} \boldsymbol{v}+\frac{1}{2} u_{0} \mathbf{q}^{\mathrm{T}} \mathbf{M}_{s r} \boldsymbol{v}+\frac{1}{2} u_{0} \boldsymbol{v}^{\mathrm{T}} \mathbf{M}_{r s} \mathbf{q} \\
& +\frac{1}{2} \dot{\mathbf{q}}^{\mathrm{T}} \mathbf{M}_{q q} \dot{\mathbf{q}}+\frac{1}{2} u_{0} \mathbf{q}^{\mathrm{T}} \mathbf{M}_{s q} \dot{\mathbf{q}}+\frac{1}{2} u_{0} \dot{\mathbf{q}}^{\mathrm{T}} \mathbf{M}_{q s} \mathbf{q}+\frac{1}{2} u_{0}^{2} \mathbf{q}^{\mathrm{T}} \mathbf{M}_{s s} \mathbf{q}
\end{aligned}
$$

where the added-mass matrices $\mathbf{M}_{r r}, \mathbf{M}_{r q}, \mathbf{M}_{q r}, \mathbf{M}_{q q}, \mathbf{M}_{r s}, \mathbf{M}_{s r}, \mathbf{M}_{s q}, \mathbf{M}_{q s}$ and $\mathbf{M}_{s s}$ are constructed from the $i, j$-entries in Table 4.1, and all these matrices are time independent. We can see that $\mathbf{M}_{r r}$ is a $6 \times 6$ rigid-body added-mass matrix and the first term in Eq. (4.12) represents the contribution of rigid-body motion terms to the 
Table 4.1: Added-mass matrix entities

\begin{tabular}{cccc}
\hline Matrix & \multicolumn{3}{c}{ Entities } \\
\hline $\mathbf{M}_{r r}$ & $M_{r r, i j}=-\rho \iint_{S_{B}} \Psi_{r i} \frac{\partial \Psi_{r j}}{\partial n} d S, \quad i=1,2, \ldots, 6$, & $j=1,2, \ldots, 6$ \\
$\mathbf{M}_{r q}$ & $M_{r q, i j}=-\rho \iint_{S_{B}} \Psi_{r i} \frac{\partial \Psi_{q j}}{\partial n} d S, \quad i=1,2, \ldots, 6$, & $j=1,2, \ldots, 2 N$ \\
$\mathbf{M}_{q r}$ & $M_{q r, i j}=-\rho \iint_{S_{B}} \Psi_{q i} \frac{\partial \Psi_{r j}}{\partial n} d S, \quad i=1,2, \ldots, 2 N$, & $j=1,2, \ldots, 6$ \\
$\mathbf{M}_{q q}$ & $M_{q q, i j}=-\rho \iint_{S_{B}} \Psi_{q i} \frac{\partial \Psi_{q j}}{\partial n} d S, \quad i=1,2, \ldots, 2 N$, & $j=1,2, \ldots, 2 N$ \\
$\mathbf{M}_{r s}$ & $M_{r s, i j}=-\rho \iint_{S_{B}} \Psi_{r i} \frac{\partial \Psi_{s j}}{\partial n} d S, \quad i=1,2, \ldots, 6$, & $j=1,2, \ldots, 2 N$ \\
$\mathbf{M}_{s r}$ & $M_{s r, i j}=-\rho \iint_{S_{B}} \Psi_{s i} \frac{\partial \Psi_{r j}}{\partial n} d S$, & $i=1,2, \ldots, 2 N$, & $j=1,2, \ldots, 6$ \\
$\mathbf{M}_{q s}$ & $M_{q s, i j}=-\rho \iint_{S_{B}} \Psi_{q i} \frac{\partial \Psi_{s j}}{\partial n} d S$, & $i=1,2, \ldots, 2 N$, & $j=1,2, \ldots, 2 N$ \\
$\mathbf{M}_{s q}$ & $M_{s q, i j}=-\rho \iint_{S_{B}} \Psi_{s i} \frac{\partial \Psi_{q j}}{\partial n} d S$, & $i=1,2, \ldots, 2 N$, & $j=1,2, \ldots, 2 N$ \\
$\mathbf{M}_{s s}$ & $M_{s s, i j}=-\rho \iint_{S_{B}} \Psi_{s i} \frac{\partial \Psi_{s j}}{\partial n} d S$, & $i=1,2, \ldots, 2 N$, & $j=1,2, \ldots, 2 N$ \\
\hline
\end{tabular}

fluid kinetic energy. The matrices $\mathbf{M}_{r q}, \mathbf{M}_{q r}$ and $\mathbf{M}_{r s}, \mathbf{M}_{s r}$ are the coupling addedmass matrices between the rigid-body and flexible velocity potentials, $\mathbf{M}_{r q}$ and $\mathbf{M}_{r s}$ are $6 \times 2 N$ matrices and $\mathbf{M}_{q r}=\mathbf{M}_{r q}^{\mathrm{T}}, \mathbf{M}_{s r}=\mathbf{M}_{r s}^{\mathrm{T}}$. The matrices $\mathbf{M}_{q q}, \mathbf{M}_{s q}, \mathbf{M}_{q s}$ and $\mathbf{M}_{s s}$ are the $2 N \times 2 N$ flexible added-mass matrices, and $\mathbf{M}_{s q}=\mathbf{M}_{s q}^{\mathrm{T}}$.

Before proceeding to develop the added-mass matrix, we explain the nature of the terms in the kinetic energy $T_{f}$. If the deformation is zero, $\dot{\mathbf{q}}=\mathbf{q}=\mathbf{0}$, and the fluid's kinetic energy reduces to the first term $\mathcal{T}_{f}=\frac{1}{2} \boldsymbol{v}^{\mathrm{T}} \mathbf{M}_{r r} \boldsymbol{v}$. This is the kinetic energy of a potential fluid in which a rigid-body vehicle is maneuvering. The second line in Eq. (4.12) effectively represents the energy of a $2 \mathrm{~N}$-DOF spring-mass-damping system associated with the elastic deformation, with a generalized added-mass matrix $\mathbf{M}_{q q}$, a damping matrix $\left(u_{0} \mathbf{M}_{s q}-u_{0} \mathbf{M}_{q s}\right)$ and a stiffness matrix $u_{0}^{2} \mathbf{M}_{s s}$. In particular, it has been demonstrated that the generalized added-mass matrix $\mathbf{M}_{q q}$ can significantly reduce the natural vibration frequencies of a vehicle moving a heavy fluid [99-102]. 
The other terms in Eq. (4.12) denote the kinetic energy due to the coupling between the rigid-body motion and elastic deformation.

Furthermore, the fluid kinetic energy can be concisely written as

$$
\mathcal{T}_{f}=\frac{1}{2}\left[\boldsymbol{v}^{\mathrm{T}}, \dot{\mathbf{q}}^{\mathrm{T}}\right] \mathbf{M}_{A T}\left[\boldsymbol{v}^{\mathrm{T}}, \dot{\mathbf{q}}^{\mathrm{T}}\right]^{\mathrm{T}}
$$

where the total added-mass matrix $\mathbf{M}_{A T}$ can be written as the summation of four matrices as

$$
\mathbf{M}_{A T}=\mathbf{M}_{A 1}+\mathbf{M}_{A 2}+\mathbf{M}_{A 3}+\mathbf{M}_{A 4}
$$

in which

$$
\begin{array}{rlr}
\mathbf{M}_{A 1}= & {\left[\begin{array}{cc}
\mathbf{M}_{r r} & \mathbf{M}_{r q} \\
\mathbf{M}_{q r} & \mathbf{M}_{q q}
\end{array}\right],} & \mathbf{M}_{A 2}=\left[\begin{array}{cccc}
\mathbf{M}_{r s} \mathbf{q} & 0 & \cdots & 0 \\
\mathbf{M}_{q s} \mathbf{q} & 0 & \cdots & 0
\end{array}\right], \\
\mathbf{M}_{A 3}=\left[\begin{array}{cc}
\mathbf{q}^{\mathrm{T}} \mathbf{M}_{s r} & \mathbf{q}^{\mathrm{T}} \mathbf{M}_{s q} \\
0 & 0 \\
\vdots & \vdots \\
0 & 0
\end{array}\right], & \mathbf{M}_{A 4}=\left[\begin{array}{cccc}
\mathbf{q}^{\mathrm{T}} \mathbf{M}_{s s} \mathbf{q} & 0 & \cdots & 0 \\
0 & 0 & \cdots & 0 \\
\vdots & \vdots & \vdots & \vdots \\
0 & 0 & \cdots & 0
\end{array}\right]
\end{array}
$$

where $\mathbf{q}^{\mathrm{T}} \mathbf{M}_{s r}$ and $\mathbf{q}^{\mathrm{T}} \mathbf{M}_{s q}$ are a $1 \times 6$ and a $1 \times 2 N$ row vectors respectively, $\mathbf{M}_{r s} \mathbf{q}$ and $\mathbf{M}_{q s} \mathbf{q}$ are a $6 \times 1$ and a $2 N \times 1$ vectors respectively, and $\mathbf{q}^{\mathrm{T}} \mathbf{M}_{s s} \mathbf{q}$ is a scalar. The total added-mass matrix $\mathbf{M}_{A T}$ is a $(6+2 N) \times(6+2 N)$ symmetric matrix dependent on the elastic generalized coordinates $\mathbf{q}$.

\subsubsection{Potential-flow aerodynamic force expressions}

The potential-flow aerodynamic forces and moments can be derived from Kirchhoff's equation, or, Lagrange's equation in terms of quasi coordinates. The corresponding elastic generalized force can be derived from Lagrange's equation in terms of general- 
ized coordinates, i.e.,

$$
\begin{aligned}
\frac{d}{d t} \frac{\partial \mathcal{T}_{f}}{\partial \mathbf{v}_{0}}+\boldsymbol{\omega}^{\times} \frac{\partial \mathcal{T}_{f}}{\partial \mathbf{v}_{0}} & =-\mathbf{F}_{A} \\
\frac{d}{d t} \frac{\partial \mathcal{T}_{f}}{\partial \boldsymbol{\omega}}+\mathbf{v}_{0}^{\times} \frac{\partial \mathcal{T}_{f}}{\partial \mathbf{v}_{0}}+\boldsymbol{\omega}^{\times} \frac{\partial \mathcal{T}_{f}}{\partial \boldsymbol{\omega}} & =-\mathbf{M}_{A} \\
\frac{d}{d t} \frac{\partial \mathcal{T}_{f}}{\partial \dot{\mathbf{q}}}-\frac{\partial \mathcal{T}_{f}}{\partial \mathbf{q}} & =-\mathbf{Q}_{A}
\end{aligned}
$$

where $\mathbf{F}_{A}, \mathbf{M}_{A}$ and $\mathbf{Q}_{A}$ are the aerodynamic forces, moments and generalized forces on the vehicle from the potential fluid respectively. They can be derived by substituting the fluid energy from Eq. (4.13) into Eqs. (4.15) to (4.17), and the results are summarized as follows.

In order to obtain a concise formulation for the aerodynamic force, moment, and generalized force, the $(6+2 N) \times(6+2 N)$ total added-mass matrix $\mathbf{M}_{A T}$ computed from Eq. (4.14), is written in block matrix form as follows

$$
\mathbf{M}_{A T}=\left[\begin{array}{lll}
\mathbf{M}_{A 11} & \mathbf{M}_{A 12} & \mathbf{M}_{A 13} \\
\mathbf{M}_{A 21} & \mathbf{M}_{A 22} & \mathbf{M}_{A 23} \\
\mathbf{M}_{A 31} & \mathbf{M}_{A 32} & \mathbf{M}_{A 33}
\end{array}\right]
$$

where $\mathbf{M}_{A 11}, \mathbf{M}_{A 12}, \mathbf{M}_{A 21}$ and $\mathbf{M}_{A 22}$ are $3 \times 3$ matrices, $\mathbf{M}_{A 13}$ and $\mathbf{M}_{A 23}$ are $3 \times 2 N$ matrices, $\mathbf{M}_{A 31}$ and $\mathbf{M}_{A 32}$ are $2 N \times 3$ matrices, $\mathbf{M}_{A 33}$ is a $2 N \times 2 N$ matrix. Similar to the mass matrix, the total added-mass matrix also depends on the generalized coordinates; thus, it is an implicit function of time. Furthermore, the $6 \times 6$ rigidbody added-mass matrix $\mathbf{M}_{r r}$ and the $6 \times(6+2 N)$ rigid-flexible coupling added-mass matrices $\mathbf{M}_{r q}$ and $\mathbf{M}_{r s}$ are written as

$$
\mathbf{M}_{r r}=\left[\begin{array}{ll}
\mathbf{M}_{r r 11} & \mathbf{M}_{r r 12} \\
\mathbf{M}_{r r 21} & \mathbf{M}_{r r 22}
\end{array}\right], \quad \mathbf{M}_{r q}=\left[\begin{array}{l}
\mathbf{M}_{r q 1} \\
\mathbf{M}_{r q 2}
\end{array}\right], \quad \mathbf{M}_{r s}=\left[\begin{array}{l}
\mathbf{M}_{r s 1} \\
\mathbf{M}_{r s 2}
\end{array}\right]
$$

where the matrices $\mathbf{M}_{r r 11}, \mathbf{M}_{r r 12}, \mathbf{M}_{r r 21}$ and $\mathbf{M}_{r r 22}$ are $3 \times 3$ matrices and $\mathbf{M}_{r q 1}, \mathbf{M}_{r q 2}$, 
$\mathbf{M}_{r s 1}$ and $\mathbf{M}_{r s 2}$ are $3 \times 2 N$ matrices.

With the above definitions, the potential-flow aerodynamic forces and moments can be obtained as

$$
\left[\begin{array}{c}
\mathbf{F}_{A} \\
\mathbf{M}_{A} \\
\mathbf{Q}_{A}
\end{array}\right]=-\left[\begin{array}{lll}
\mathbf{M}_{A 11} & \mathbf{M}_{A 12} & \mathbf{M}_{A 13} \\
\mathbf{M}_{A 21} & \mathbf{M}_{A 22} & \mathbf{M}_{A 23} \\
\mathbf{M}_{A 31} & \mathbf{M}_{A 32} & \mathbf{M}_{A 33}
\end{array}\right]\left[\begin{array}{c}
\dot{\mathbf{v}}_{0} \\
\dot{\boldsymbol{\omega}} \\
\ddot{\mathbf{q}}
\end{array}\right]+\left[\begin{array}{l}
\mathbf{F}_{A, n o n}\left(\mathbf{v}_{0}, \boldsymbol{\omega}, \mathbf{q}, \dot{\mathbf{q}}\right) \\
\mathbf{M}_{A, n o n}\left(\mathbf{v}_{0}, \boldsymbol{\omega}, \mathbf{q}, \dot{\mathbf{q}}\right) \\
\mathbf{Q}_{A, n o n}\left(\mathbf{v}_{0}, \boldsymbol{\omega}, \mathbf{q}, \dot{\mathbf{q}}\right)
\end{array}\right]
$$

where the first term is related to the linear acceleration $\dot{\mathbf{v}}_{0}$, angular acceleration $\dot{\boldsymbol{\omega}}$ and the elastic generalized acceleration $\ddot{\mathbf{q}}$, while the second term contains nonlinear functions of the rigid-body motion velocities $\mathbf{v}_{0}, \boldsymbol{\omega}$, the generalized coordinate $\mathbf{q}$, and the generalized velocity $\dot{\mathbf{q}}$. To incorporate the potential-flow aerodynamics, the first term is added to the left hand side of the equations of motion (3.11) so that the mass matrix $\mathbf{M}_{\text {sys }}$ is replaced with $\mathbf{M}_{\text {sys }}+\mathbf{M}_{A T}$; while $\mathbf{F}_{A, n o n}, \mathbf{M}_{A, \text { non }}$ and $\mathbf{Q}_{A, \text { non }}$ are incorporated into the right hand side of the equations of motion. These force and moment terms are given as follows

$$
\begin{aligned}
& \mathbf{F}_{A, n o n}=-\boldsymbol{\omega}^{\times}\left(\mathbf{M}_{r r 11} \mathbf{v}_{0}+\mathbf{M}_{r r 12} \boldsymbol{\omega}\right) \\
&-\boldsymbol{\omega}^{\times} \mathbf{M}_{r q 1} \dot{\mathbf{q}} \\
&-u_{0} \boldsymbol{\omega}^{\times} \mathbf{M}_{r s 1} \mathbf{q}-u_{0} \mathbf{M}_{r s 1} \dot{\mathbf{q}}-\left[\boldsymbol{v}^{\mathrm{T}} \mathbf{M}_{r s} \dot{\mathbf{q}}+\dot{\mathbf{q}}^{\mathrm{T}} \mathbf{M}_{s q} \dot{\mathbf{q}}+2 u_{0} \dot{\mathbf{q}}^{\mathrm{T}} \mathbf{M}_{s s} \mathbf{q}, 0,0\right]^{\mathrm{T}} \\
&-\boldsymbol{\omega}^{\times}\left[\boldsymbol{v}^{\mathrm{T}} \mathbf{M}_{r s} \mathbf{q}+\mathbf{q}^{\mathrm{T}} \mathbf{M}_{s q} \dot{\mathbf{q}}+u_{0} \mathbf{q}^{\mathrm{T}} \mathbf{M}_{s s} \mathbf{q}, 0,0\right]^{\mathrm{T}} \\
& \mathbf{M}_{A, n o n}=-\mathbf{v}_{0}^{\times}\left(\mathbf{M}_{r r 11} \mathbf{v}_{0}+\mathbf{M}_{r r 12} \boldsymbol{\omega}\right)-\boldsymbol{\omega}^{\times}\left(\mathbf{M}_{r r 21} \mathbf{v}_{0}+\mathbf{M}_{r r 22} \boldsymbol{\omega}\right) \\
&-\mathbf{v}_{0}^{\times} \mathbf{M}_{r q 1} \dot{\mathbf{q}}-\boldsymbol{\omega}^{\times} \mathbf{M}_{r q 2} \dot{\mathbf{q}} \\
&-u_{0} \mathbf{M}_{r s 2} \dot{\mathbf{q}}-u_{0} \boldsymbol{\omega}^{\times} \mathbf{M}_{r s 2} \mathbf{q} \\
&-\mathbf{v}_{0}^{\times}\left(u_{0} \mathbf{M}_{r s 1} \mathbf{q}+\left[\boldsymbol{v}^{\mathrm{T}} \mathbf{M}_{r s} \mathbf{q}+\mathbf{q}^{\mathrm{T}} \mathbf{M}_{s q} \dot{\mathbf{q}}+u_{0} \mathbf{q}^{\mathrm{T}} \mathbf{M}_{s s} \mathbf{q}, 0,0\right]^{\mathrm{T}}\right) \\
&=-u_{0} \mathbf{M}_{q s} \dot{\mathbf{q}}+u_{0} \mathbf{M}_{s q} \dot{\mathbf{q}}+u_{0}^{2} \mathbf{M}_{s s} \mathbf{q}+u_{0} \mathbf{M}_{s r} \boldsymbol{v} \\
& \mathbf{Q}_{A, n o n}
\end{aligned}
$$


The first lines in the expressions of $\mathbf{F}_{A, \text { non }}$ and $\mathbf{M}_{A, \text { non }}$ contain the force and moment terms due to the rigid-body motion; the second lines contain the terms due to the coupling between the deflection rates and rigid-body motion; and the third and fourth lines include the terms related to the bending slopes. If the elastic generalized coordinates and velocities are zero, the external forces and moments are reduced to those of a rigid-body vehicle in Eq. (2.9).

The first two terms in $\mathbf{Q}_{A, n o n}$ denote the aerodynamic damping effects on the elastic deformation; the term $u_{0}^{2} \mathbf{M}_{s s} \mathbf{q}$ is an effective aerodynamic stiffness term; the last term represents the influence of the rigid-body motion on the deformation.

\subsubsection{Computation of the added-mass matrix}

In order to compute the total added-mass matrix $\mathbf{M}_{A T}$, the matrices defined in Table 4.1 must be evaluated. In fact, the added-mass matrix related to the pure rigidbody motion, $\mathbf{M}_{r r}$, can be computed with the approach discussed in Section 2.3.1.2, including the contributions of the hull and the fins. For the other added-mass matrices, $\mathbf{M}_{r q}, \mathbf{M}_{q r}, \mathbf{M}_{q q}, \mathbf{M}_{r s}, \mathbf{M}_{s r}, \mathbf{M}_{q s}, \mathbf{M}_{s q}$ and $\mathbf{M}_{s s}$, the Laplace equations must be solved to obtain $\Psi_{r i}, \Psi_{q i}$ and $\Psi_{s i}$; then the elements of each added-mass matrix can be evaluated by the integrals defined in Table 4.1. For a body of irregular shape, the Laplace equation can be solved with numerical approaches. In this work, the analytical solution of the Laplace equation for an ellipsoid of revolution is applied to obtain $\Psi_{r i}, \Psi_{q i}$ and $\Psi_{s i}$. The main steps of this solution are presented in the following.

Lamb [25] derived $\Psi_{r i}$ for an ellipsoid of revolution, in terms of the ellipsoidal coordinates, Legendre function and associated Legendre function, and estimated the hull contribution to the added-mass matrix of a rigid-body airship, i.e., $\mathbf{M}_{A H}$ in Eq. (2.10). Macagno and Landweber [100] extended Lamb's results to solve the Laplace equation in order to predict the natural frequencies of the ship vibration in water. They found that the velocity potential for an ellipsoid with arbitrary boundary conditions can be 
written as

$$
\Psi_{k i}=\sum_{n=0}^{\infty} \sum_{p=0}^{n}\left[B_{n}^{p} \sin (p \vartheta)+C_{n}^{p} \cos (p \vartheta)\right] P_{n}^{p}(\mu) Q_{n}^{p}(\zeta), \quad k=r, q, s
$$

where $P_{n}^{p}(\mu)$ and $Q_{n}^{p}(\zeta)$ are respectively the Legendre function and associated Legendre function, of degree $n$ and order $p$. The coefficients $B_{n}^{p}$ and $C_{n}^{p}$ are determined from the boundary conditions and will be discussed later. The ellipsoidal coordinates $(\zeta, \mu, \vartheta)$ can be converted to Cartesian coordinates as below with parameters $a$ and $e$ as the semi major axis and the eccentricity of the ellipsoid

$$
\begin{array}{ll}
x=a e \mu \zeta & 1 \leq \zeta \leq \infty \\
y=a e \sqrt{1-\mu^{2}} \sqrt{\zeta^{2}-1} \cos \vartheta & -1 \leq \mu \leq 1 \\
z=a e \sqrt{1-\mu^{2}} \sqrt{\zeta^{2}-1} \sin \vartheta & 0 \leq \vartheta \leq 2 \pi
\end{array}
$$

An ellipsoidal coordinate system is composed of three families of orthogonal surfaces: the ellipsoid surfaces and their associated hyperboloids and planes. The surfaces $\zeta=$ constant are ellipsoids of revolution, the surfaces $\mu=$ constant are hyperboloids and both ellipsoid and hyperboloid families have common foci at $( \pm a e, 0,0)$. The surfaces $\vartheta=$ constant are planes through the $x$ axis that are orthogonal to the first two families. Particularly, $\zeta=1 / e$ denotes an ellipsoid of a semi major axis $a$ and an eccentricity $e$, and the boundary condition of the velocity potentials $\Psi_{r i}, \Psi_{q i}$ and $\Psi_{s i}$ will be converted to ellipsoidal coordinates over the surface of this ellipsoid.

The coefficients $B_{n}^{p}$ and $C_{n}^{p}$ in Eq. (4.23) can be computed by Macagno and Landweber's formula [100]. For this purpose, the boundary conditions of the velocity potentials $\Psi_{r i}, \Psi_{q i}$ and $\Psi_{s i}$ presented in Eqs. (4.9) to (4.11) are rewritten in ellipsoidal coordinates as follows

$$
\frac{\partial \Psi_{k i}}{\partial \zeta}=a e F(\mu, \vartheta)=u_{b, k i} \frac{\partial x}{\partial \zeta}+v_{b, k i} \frac{\partial y}{\partial \zeta}+w_{b, k i} \frac{\partial z}{\partial \zeta}, \quad \text { on } \zeta=\frac{1}{e}, \quad k=r, q, s
$$


Table 4.2: Velocity distribution corresponding to boundary conditions

\begin{tabular}{cccc}
\hline Velocity potential & $u_{b, k i}$ & $v_{b, k i}$ & $w_{b, k i}$ \\
\hline$\Psi_{r 1}$ & 1 & 0 & 0 \\
$\Psi_{r 2}$ & 0 & 1 & 0 \\
$\Psi_{r 3}$ & 0 & 0 & 1 \\
$\Psi_{r 4}$ & 0 & $-z$ & $y$ \\
$\Psi_{r 5}$ & $z$ & 0 & $-x$ \\
$\Psi_{r 6}$ & $-y$ & $x$ & 0 \\
$\Psi_{q i}$ & $\Phi_{x i}$ & $\Phi_{y i}$ & $\Phi_{z i}$ \\
$\Psi_{s i}$ & $-\Phi_{x i}^{\prime}$ & $-\Phi_{y i}^{\prime}$ & $-\Phi_{z i}^{\prime}$ \\
\hline
\end{tabular}

where $u_{b, k i}, v_{b, k i}$ and $w_{b, k i}$ represent the velocity distribution corresponding to the boundary condition of velocity potential $\Psi_{k i}$, as listed in 4.2. Substituting $x, y$ and $z$ from Eqs. (4.24) to (4.26) leads to

$$
F(\mu, \vartheta)=u_{b, k i} \mu+\frac{\sqrt{1-\mu^{2}}}{\sqrt{1-e^{2}}}\left(v_{b, k i} \cos \vartheta+w_{b, k i} \sin \vartheta\right), \quad \text { on } \zeta=\frac{1}{e}, \quad k=r, q, s
$$

Then $B_{n}^{p}$ and $C_{n}^{p}$ can be computed as [100]

$$
\begin{aligned}
& B_{n}^{p}= \begin{cases}0 & \text { if } \quad p=0 \\
\frac{2 n+1}{2 \pi} \frac{(n-p) !}{(n+p) !} \frac{a e}{\partial Q_{n}^{p} / \partial \zeta} \int_{0}^{2 \pi} \int_{1}^{-1} F(\mu, \vartheta) P_{n}^{p}(\mu) \sin (p \vartheta) d \mu d \vartheta & \text { if } \quad p \geq 1\end{cases} \\
& C_{n}^{p}= \begin{cases}\frac{2 n+1}{4 \pi} \frac{a e}{\partial Q_{n}^{p} / \partial \zeta} \int_{0}^{2 \pi} \int_{1}^{-1} F(\mu, \vartheta) P_{n}^{p}(\mu) d \mu d \vartheta & \text { if } \quad p=0 \\
\frac{2 n+1}{2 \pi} \frac{(n-p) !}{(n+p) !} \frac{a e}{\partial Q_{n}^{p} / \partial \zeta} \int_{0}^{2 \pi} \int_{1}^{-1} F(\mu, \vartheta) P_{n}^{p}(\mu) \cos (p \vartheta) d \mu d \vartheta & \text { if } \quad p \geq 1\end{cases}
\end{aligned}
$$

Equation (4.23) gives the velocity potential at any point of the fluid and the coefficients $B_{n}^{p}$ and $C_{n}^{p}$ are independent of $\zeta$ since they are computed on a specific ellipsoid surface $\zeta=\frac{1}{e}$.

Macagno and Landweber [100] also gave the expression for the fluid kinetic energy using ellipsoidal coordinates and this can be extended to compute the entities in the 
added-mass matrices. That is, the added-mass entities defined in Table 4.1 can be rewritten in ellipsoidal coordinates as follows

$$
m_{k_{\mathrm{I}} k_{\mathrm{II}}, i j}=-\left.\rho a e\left(\frac{1}{e^{2}}-1\right) \int_{0}^{2 \pi} \int_{-1}^{1}\left[\Psi_{k_{\mathrm{I}}, i} \frac{\partial \Psi_{k_{\mathrm{II}}, j}}{\partial \zeta}\right]\right|_{\zeta=\frac{1}{e}} d \mu d \vartheta, \quad k_{\mathrm{I}}, k_{\mathrm{II}}=r, q, s
$$

where $\partial \Psi_{k_{\mathrm{II}}, j} / \partial \zeta$ is obtained from Eq. (4.23) as

$$
\frac{\partial \Psi_{k_{\mathrm{II}}, j}}{\partial \zeta}=\sum_{n=0}^{\infty} \sum_{p=0}^{n}\left[B_{n}^{p} \sin (p \vartheta)+C_{n}^{p} \cos (p \vartheta)\right] P_{n}^{p}(\mu) \frac{\partial Q_{n}^{p}(\zeta)}{\partial \zeta}, \quad k_{\mathrm{II}}=r, q, s
$$

Following Macagno and Landweber's method to solve $\Psi_{r i}, \Psi_{q i}$ and $\Psi_{s i}$, the procedure to evaluate the added-mass matrices $\mathbf{M}_{r q}, \mathbf{M}_{q r}, \mathbf{M}_{q q}, \mathbf{M}_{r s}, \mathbf{M}_{s r}, \mathbf{M}_{q s}, \mathbf{M}_{s q}$ and $\mathbf{M}_{s s}$ is summarized as follows:

1. Rewrite the corresponding boundary conditions in Eqs. (4.9) to (4.11) in terms of ellipsoidal coordinates, using Eq. (4.27).

2. Compute $B_{n}^{p}$ and $C_{n}^{p}$ according to the boundary condition, using Macagno and Landweber's formula in Eqs. (4.29) and (4.30).

3. Obtain $\Psi_{r i}(i=1,2, \ldots, 6), \Psi_{q i}(i=1,2, \ldots, 2 N)$, $\Psi_{s i}(i=1,2, \ldots, 2 N)$ and $\partial \Psi_{r j} / \partial \zeta, \partial \Psi_{q j} / \partial \zeta, \partial \Psi_{s j} / \partial \zeta$ from Eqs. (4.23) and (4.32), over the ellipsoid surface, by evaluating them at $\zeta=1 / e$.

4. Evaluate the components of the matrices $\mathbf{M}_{r q}, \mathbf{M}_{q r}, \mathbf{M}_{q q}, \mathbf{M}_{r s}, \mathbf{M}_{s r}, \mathbf{M}_{q s}, \mathbf{M}_{s q}$ and $\mathbf{M}_{s s}$, according to Eq. (4.31).

Once the added-mass matrices are computed, the potential-flow aerodynamic forces and moments can be obtained from Eqs. (4.19) to (4.22). 


\subsection{Viscous Effect on the Hull}

As discussed in Section 2.3.2, the viscous effect on the hull starts from a position $\varepsilon_{V}$ and was calculated previously for an airship modeled as a rigid body. To extend this method for an elastic airship, the corresponding aerodynamic normal force distribution along the centerline is first predicted by using the local velocity of a deformed airship, i.e.,

$$
\mathbf{f}_{V}=f_{V N}\left[0, \frac{-v_{V, d}}{\sqrt{v_{V, d}^{2}+w_{V, d}^{2}}}, \frac{-w_{V, d}}{\sqrt{v_{V, d}^{2}+w_{V, d}^{2}}}\right]^{\mathrm{T}}, \quad \text { from } \varepsilon_{V} \text { to the end }
$$

where

$$
f_{V N}=-q_{0} \sin 2 \gamma \cdot\left(k_{2}-k_{1}\right) \frac{d S}{d \varepsilon}+q_{0} \eta C_{D C} \sin ^{2} \gamma \cdot 2 R
$$

and recalling $k_{1}, k_{2}, \eta, C_{D C}, S$ and $R$ from Section 2.3.2, $u_{V, d}, v_{V, d}$ and $w_{V, d}$ denoting

the local velocity at the position $\varepsilon_{V}$ on a deformed airship computed using Eq. (4.4). These velocity components are also used to compute the dynamic pressure $q_{0}$ and the angle between centerline and velocity vector $\gamma$ for a flexible airship, i.e.,

$$
\gamma=\tan ^{-1}\left(\frac{\sqrt{v_{V, d}^{2}+w_{V, d}^{2}}}{u_{V, d}}\right)
$$

The total external force can be readily computed by integrating $\mathbf{f}_{V}$ from $\varepsilon_{V}$ to the end of the hull as

$$
\mathbf{F}_{V}=F_{V N}\left[0, \frac{-v_{V, d}}{\sqrt{v_{V, d}^{2}+w_{V, d}^{2}}}, \frac{-w_{V, d}}{\sqrt{v_{V, d}^{2}+w_{V, d}^{2}}}\right]^{\mathrm{T}}
$$

in which $F_{V N}$ can be computed using Eq. (2.15), because only $d S / d \varepsilon$ and $2 R$ in $f_{V N}$ depend on the location. The external moment is now computed by integrating 
$(\mathbf{r}+\mathbf{u})^{\times} \mathbf{f}_{V}$ from $\varepsilon_{V}$ to the end. After some algebra, we obtain

$$
\mathbf{M}_{V}=\left[M_{V x}, M_{V N} \frac{w_{V, d}}{\sqrt{v_{V, d}^{2}+w_{V, d}^{2}}}, M_{V N} \frac{-v_{V, d}}{\sqrt{v_{V, d}^{2}+w_{V, d}^{2}}}\right]^{\mathrm{T}}
$$

where $M_{V N}$ can be computed using Eq. (2.18) and

$$
\begin{aligned}
M_{V x}= & -q_{0} \sin 2 \gamma \cdot\left(k_{2}-k_{1}\right) \sum_{i=1}^{2 N} q_{i} \mathbf{I}_{V 1, i}^{\mathrm{T}}\left[0, \frac{-w_{V, d}}{\sqrt{v_{V, d}^{2}+w_{V, d}^{2}}}, \frac{v_{V, d}}{\sqrt{v_{V, d}^{2}+w_{V, d}^{2}}}\right]^{\mathrm{T}} \\
& +q_{0} \eta C_{D C} \sin ^{2} \gamma \cdot \sum_{i=1}^{2 N} q_{i} \mathbf{I}_{V 2, i}^{\mathrm{T}}\left[0, \frac{-w_{V, d}}{\sqrt{v_{V, d}^{2}+w_{V, d}^{2}}}, \frac{v_{V, d}}{\sqrt{v_{V, d}^{2}+w_{V, d}^{2}}}\right]^{\mathrm{T}}
\end{aligned}
$$

Two new shape function integrals are employed in Eq. (4.38) as

$$
\mathbf{I}_{V 1, i}=\int_{\varepsilon_{V}}^{L} \frac{d S}{d \varepsilon} \boldsymbol{\Phi}_{\mathbf{i}} d \varepsilon, \quad \mathbf{I}_{V 2, i}=\int_{\varepsilon_{V}}^{L} 2 R \boldsymbol{\Phi}_{\mathbf{i}} d \varepsilon, \quad i=1,2, \ldots, 2 N
$$

The roll moment $M_{V x}$ results from the term $\mathbf{u}^{\times} \mathbf{f}_{V}$. This moment arm $\mathbf{u}$ reflects the deflection of the centerline from the $x$ axis of the undeformed airship, as shown in Fig. 4.1.

The elastic generalized force of the $i^{\text {th }}$ shape function is computed as $Q_{V i}=$ $\int_{\varepsilon_{V}}^{L} \mathbf{f}_{V}^{\mathrm{T}} \boldsymbol{\Phi} d \varepsilon$. Substituting $\mathbf{f}_{V}$ from Eq. (4.34), we have

$$
\begin{aligned}
Q_{V i}= & -q_{0} \sin 2 \gamma \cdot\left(k_{2}-k_{1}\right) \cdot q_{i} \mathbf{I}_{V 1, i}^{\mathrm{T}}\left[0, \frac{-v_{V, d}}{\sqrt{v_{V, d}^{2}+w_{V, d}^{2}}}, \frac{-w_{V, d}}{\sqrt{v_{V, d}^{2}+w_{V, d}^{2}}}\right]^{\mathrm{T}} \\
& +q_{0} \eta C_{D C} \sin ^{2} \gamma \cdot q_{i} \mathbf{I}_{V 2, i}^{\mathrm{T}}\left[0, \frac{-v_{V, d}}{\sqrt{v_{V, d}^{2}+w_{V, d}^{2}}}, \frac{-w_{V, d}}{\sqrt{v_{V, d}^{2}+w_{V, d}^{2}}}\right]^{\mathrm{T}}
\end{aligned}
$$

We can see that the influence of the flexibility on the viscous effect is incorporated through the local velocity $\mathbf{v}_{V, d}$ in the computation of the dynamic pressure and the angle $\gamma$, as well as by adding a roll moment related to the centerline deflection. 


\subsection{Force Acting on the Fins}

The distribution of the force acting on the fins can be predicted using Eq. (2.20), then the force per unit length can be written as

$$
\mathbf{f}_{F}=f_{F N}\left[0, \cos \phi_{F},-\sin \phi_{F}\right]^{\mathrm{T}}
$$

where

$$
f_{F N}=q_{0} \frac{C_{L \alpha}}{C_{l \alpha}} \alpha_{F} \int_{R}^{b} \Delta C_{p \alpha}(x, s)\left(1+\frac{R^{2}}{s^{2}}\right) d s
$$

and $C_{L \alpha} / C_{l \alpha}$ and $\Delta C_{p \alpha}$ are defined as before in Section 2.3.3. For a flexible airship, the geometric angle of attack $\alpha_{F}$ used in the evaluation of $f_{F N}$ is computed with the velocity components $u_{d}(c / 4)$ and $v_{n d}(c / 4)$ at the center of the $1 / 4$-chord (plotted in Fig. 2.9), where the subscript $d$ denotes the local velocity components on a deformed airship computed from Eq. (4.4). That is

$$
\alpha_{F}=\tan ^{-1}\left[\frac{v_{n d}(c / 4)}{u_{d}(c / 4)}\right]
$$

The total normal force on the fin can then be obtained using Eq. (2.23) with $\alpha_{F}$ from Eq. (4.42). The corresponding moment is computed by integrating $(\mathbf{r}+\mathbf{u})^{\times} \mathbf{f}_{F}$ over the exposed fin area as follows

$$
\mathbf{M}_{F}=\left[M_{F x}, M_{F N} \sin \Phi_{F}, M_{F N} \cos \Phi_{F}\right]^{\mathrm{T}}
$$

where $M_{F N}$ is calculated using Eq. (2.25) and $\Phi_{F}$ is the angle from the oxz plane to the fin plane (see Fig. 2.9). The roll moment $M_{F x}$ results from the term $\mathbf{u}^{\times} \mathbf{f}_{F}$ and is 
obtained as

$$
\begin{aligned}
M_{F x}= & -q_{0} \frac{C_{L \alpha}}{C_{l \alpha}} \alpha_{F} \int_{x_{F s}}^{x_{F e}} \int_{R}^{b} s \Delta C_{p \alpha}(x, s)\left(1+\frac{R^{2}}{s^{2}}\right) d s d x \\
& -q_{0} \frac{C_{L \alpha}}{C_{l \alpha}} \alpha_{F} \sum_{i=1}^{2 N} q_{i} \mathbf{I}_{F i}^{\mathrm{T}}\left[0, \cos \phi_{F}, \sin \phi_{F}\right]^{\mathrm{T}}
\end{aligned}
$$

The shape function integrals $\mathbf{I}_{F i}$ required in Eq. (4.44) are defined as

$$
\mathbf{I}_{F i}=\int_{x_{F s}}^{x_{F e}} \int_{R}^{b} \Delta C_{p \alpha}(x, s)\left(1+\frac{R^{2}}{s^{2}}\right) \boldsymbol{\Phi}_{i} d s d x, \quad i=1,2, \ldots, 2 N
$$

The elastic generalized force is computed as $Q_{F i}=\int_{x_{F s}}^{x_{F e}} \mathbf{f}_{F}^{\mathrm{T}} \mathbf{\Phi} d x$, or,

$$
Q_{F i}=-q_{0} \frac{C_{L \alpha}}{C_{l \alpha}} \alpha_{F} \mathbf{I}_{F i}^{\mathrm{T}}\left[0, \cos \phi_{F}, \sin \phi_{F}\right]^{\mathrm{T}}, \quad i=1,2, \ldots, 2 N
$$

We can see that the influence of the flexibility on the fin forces is incorporated through the local velocity components $v_{n d}(c / 4)$ and $u_{d}(c / 4)$ at the center of $1 / 4$-chord for the computation of the geometric angle of attack $\alpha_{F}$, as well as an additional roll moment as the second term in Eq. (4.44). This roll moment is because of the deflection of the centerline from the $x$ axis of the undeformed airship

\subsection{Force Acting on the Hull due to the Fins}

The extra normal force per unit length on the hull due to the fins $\mathbf{f}_{H(F)}$ can be predicted using Eq. (2.26). For a flexible airship, the fin-induced downwash should be computed with the geometric angle of attack $\alpha_{F}$ from Eq. (4.42).

The external force on the hull due to the fins is obtained by integrating $\mathbf{f}_{H(F)}$ over the hull length, or,

$$
\mathbf{F}_{H(F)}=F_{H(F)}\left[0, \cos \Phi_{F},-\sin \Phi_{F}\right]^{\mathrm{T}}
$$


where $F_{H(F)}=2 q_{0} \alpha_{F} \int_{L} \pi R^{2} C_{H(F)} d x$ and

$$
C_{H(F)}=\frac{C_{L \alpha}}{C_{l \alpha}} \frac{d}{d x} \int_{x_{F s}}^{x_{F e}} \int_{R}^{b}\left[8 \pi d_{F}\left(d_{F}-x+x_{F}\right)\right]^{-1} \Delta C_{p \alpha}\left(1+\frac{R^{2}}{s^{2}}\right) d s d x_{F}
$$

Recall that $d_{F}=\sqrt{\left(x-x_{F}\right)^{2}+s^{2}}$. The corresponding external moment is computed by integrating $(\mathbf{r}+\mathbf{u})^{\times} \mathbf{f}_{H(F)}$ over the hull, i.e.,

$$
\mathbf{M}_{H(F)}=\left[M_{H(F) x}, M_{H(F) N} \sin \Phi_{F}, M_{H(F) N} \cos \Phi_{F}\right]^{\mathrm{T}}
$$

where

$$
\begin{aligned}
M_{H(F) x} & =-2 q_{0} \alpha_{F} \sum_{i=1}^{2 N} q_{i} \mathbf{I}_{H(F), i}^{\mathrm{T}}\left[0, \cos \phi_{F}, \sin \phi_{F}\right]^{\mathrm{T}} \\
M_{H(F) N} & =2 q_{0} \alpha_{F} \int_{L} \pi R^{2} C_{H(F)} x d x
\end{aligned}
$$

The roll moment in Eq. (4.49) is obtained from the term $\mathbf{u}^{\times} \mathbf{f}_{H(F)}$. The new shape function integrals $\mathbf{I}_{H(F), i}$ employed in Eq. (4.49) are defined as

$$
\mathbf{I}_{H(F), i}=\int_{L} \pi R^{2} C_{H(F)} \boldsymbol{\Phi}_{i} d x, \quad i=1,2, \ldots, 2 N
$$

Lastly, the corresponding elastic generalized force is computed as

$$
Q_{H(F), i}=2 q_{0} \alpha_{F} \mathbf{I}_{H(F), i}^{\mathrm{T}}\left[0, \cos \phi_{F}, \sin \phi_{F}\right]^{\mathrm{T}}, \quad i=1,2, \ldots, 2 N
$$

Similar to the fin forces, the influence of the flexibility on $\mathbf{F}_{H(F)}$ and $\mathbf{M}_{H(F)}$ is incorporated through the local velocity in the computation of the geometric angle of attack, as well as an additional roll moment due to the deflection of the centerline. 


\subsection{Axial Drag}

The axial drag force acting on the hull and on a fin are modeled as concentrated forces at the C.V. and at the centers of the fin's 1/4-chord respectively. The external axial drag forces, $\mathbf{F}_{\text {Axial }, H}$ and $\mathbf{F}_{\text {Axial,F}}$, can be computed using Eq. (2.28), with the dynamic pressure and the angles of attack $\alpha$ and $\alpha_{F}$ calculated from the velocity components in Eq. (4.4). In addition, the elastic displacement should be considered in the moment computation. Recall from Section 3.4 that the elastic displacement of the C.V. is computed as $\mathbf{u}_{V}=\sum_{i=1}^{2 N} q_{i}(t) \mathbf{I}_{A S, i}$ with $\mathbf{I}_{A S, i}$ defined in Eq. (3.38), so that the moment due to the axial drag on the hull is

$$
\mathbf{M}_{A x i a l, H}=\mathbf{u}_{V}^{\times} \mathbf{F}_{A x i a l, H}=\sum_{i=1}^{2 N} q_{i}(t) \mathbf{I}_{A S, i}^{\times} \mathbf{F}_{A x i a l, H}
$$

Similarly, the moment due to the axial drag on a fin is calculated as

$$
\mathbf{M}_{\text {Axial }, F}=\mathbf{r}(c / 4)^{\times} \mathbf{F}_{\text {Axial }, F}+\sum_{i=1}^{2 N} q_{i} \boldsymbol{\Phi}_{i}(c / 4)^{\times} \mathbf{F}_{\text {Axial }, F}
$$

where $\mathbf{r}(c / 4)$ denotes the position vector of the center of $1 / 4$-chord from the bodyframe origin and $\boldsymbol{\Phi}_{i}(c / 4)$ is the $i^{\text {th }}$ shape function at this point. The second term reflects the fact that the elastic deflection at $\mathbf{r}(c / 4)$ should be included in the moment arm in the moment calculation. Since the axial drag is along the $x$ axis, while the modeled flexibility is due to bending only, the corresponding elastic forces are zero, i.e., $Q_{A x i a l, H i}=Q_{A x i a l, F i}=0$. 


\subsection{Force and Moment due to Control Surface De- flection}

The force due to the control surface deflection is modeled as a concentrated force acting at a position $\mathbf{r}_{\delta}$ on the undeformed body. The external force $\mathbf{F}_{\delta}$ and the position $\mathbf{r}_{\delta}$ can be computed using Eqs. (2.29), (2.31), and (2.32), as well as a dynamic pressure calculated from the local velocity on a deformed airship from Eq. (4.4). The external moment is calculated as

$$
\mathbf{M}_{\delta}=\mathbf{r}_{\delta}^{\times} \mathbf{F}_{\delta}+\sum_{i=1}^{2 N} q_{i}(t) \boldsymbol{\Phi}_{i}\left(\mathbf{r}_{\delta}\right)^{\times} \mathbf{F}_{\delta}
$$

where $\boldsymbol{\Phi}_{i}\left(\mathbf{r}_{\delta}\right)$ is the $i^{\text {th }}$ shape function at $\mathbf{r}_{\delta}$. The second term reflects the fact that the elastic deflection at $\mathbf{r}_{\delta}$ should be incorporated in the moment arm when computing the external moment. The elastic force due to the control surface deflection is then obtained as

$$
Q_{\delta i}=\mathbf{F}_{\delta}^{\mathrm{T}} \boldsymbol{\Phi}_{i}\left(\mathbf{r}_{\delta}\right)
$$

Finally, the rudder and elevator forces and moments $\mathbf{F}_{\delta}, \mathbf{M}_{\delta}$ and $\mathbf{Q}_{\delta}$ are included in $\mathbf{F}_{C}, \mathbf{M}_{C}$ and $\mathbf{Q}_{C}$ respectively in the equations of motion (3.11). 


\section{Chapter 5}

\section{Dynamics Model of Flexible}

\section{Airships: Implementation and Simulation}

Chapters 3 and 4 present a theoretical framework for the dynamics modeling of flexible airships, with regard to the equations of motion and aerodynamics computation, respectively. This model is implemented in the present chapter to simulate the dynamics of the elastic Skyship-500 airship. The chapter is organized as follows. The implementation of the dynamics model is first explained and the physical parameters of the simulated airship are given. Then, the following simulation results are presented: the normal mode shapes of the airship, selected shape function integrals involved in the calculation of inertial and aerodynamic forces, the airship's time responses to elevator/rudder input with and without control, and the frequency responses to the control surface deflections based on the linearized model.

The SI units (length $[\mathrm{m}]$, time $[\mathrm{s}]$, mass $[\mathrm{kg}]$ ) are employed in the presentation of all simulation results. 


\subsection{Implementation}

\subsubsection{Summary of the dynamics model}

Before presenting the simulation results, we give a summary of the theoretical model in Chapters 3 and 4 . The dynamics model of a flexible airship is represented by a single set of nonlinear ordinary differential equations (3.11). The mass matrix and the inertial, gravity and aerostatic forces are investigated in Sections 3.3 and 3.4; various aerodynamic effects are discussed in Sections 4.2 to 4.7. Tables 5.1 and 5.2 provide a summary of the computational formulations of each term, the involved shape function integrals and the information required to calculate these integrals. Note that these shape function integrals are all independent of time and can be evaluated before we solve the dynamics equations of motion.

Table 5.1: Summary of mass matrix and inertial, gravity and aerostatic forces

\begin{tabular}{|c|c|c|c|}
\hline Term & Formulation & $\begin{array}{c}\text { Shape function } \\
\text { integrals }\end{array}$ & $\begin{array}{c}\text { Required } \\
\text { information }\end{array}$ \\
\hline $\begin{array}{c}\text { Mass matrix } \\
\mathbf{M}_{\text {sys }}\end{array}$ & $(3.21)$ to $(3.23)$ & $\begin{array}{c}\mathbf{p}_{i}, \mathbf{J}_{r u, i}^{\prime}, \mathbf{J}_{u u, i j}^{\prime \prime} \\
\mathbf{H}, \mathbf{H}_{u, i}^{\prime}, \mathbf{M}_{e} \\
\end{array}$ & $\bar{m}$ \\
\hline $\begin{array}{l}\text { Elastic force } \\
\qquad \mathbf{S}_{E}\end{array}$ & $(3.13)$ & K & $E I$ \\
\hline $\begin{array}{l}\text { Inertial force } \\
\left(\mathbf{F}_{I}, \mathbf{M}_{I}, \mathbf{Q}_{I}\right)\end{array}$ & $(3.30)$ & $\begin{array}{c}\mathbf{p}_{i}, \mathbf{J}_{r u, i}^{\prime}, \mathbf{J}_{u u, i j}^{\prime \prime} \\
\mathbf{H}, \mathbf{H}_{u, i}^{\prime}\end{array}$ & $\bar{m}$ \\
\hline $\begin{array}{l}\text { Gravity force } \\
\left(\mathbf{F}_{G}, \mathbf{M}_{G}, \mathbf{Q}_{G}\right)\end{array}$ & $(2.5),(3.35),(3.36)$ & $\mathbf{p}_{i}$ & $\bar{m}$ \\
\hline $\begin{array}{l}\text { Aerostatic force } \\
\left(\mathbf{F}_{A S}, \mathbf{M}_{A S}, \mathbf{Q}_{A S}\right)\end{array}$ & $(2.7),(3.37),(3.39)$ & $\mathbf{I}_{A S, i}$ & $S$ \\
\hline
\end{tabular}


Table 5.2: Summary of aerodynamic forces

\begin{tabular}{|c|c|c|c|}
\hline Term & Formulation & $\begin{array}{l}\text { Shape function } \\
\text { integrals }\end{array}$ & $\begin{array}{c}\text { Required } \\
\text { information }\end{array}$ \\
\hline \multicolumn{4}{|c|}{ Potential-flow aerodynamic force } \\
\hline $\mathbf{F}_{A}$ & $(4.19),(4.20)$ & $\mathbf{M}_{r q}, \mathbf{M}_{r s}, \mathbf{M}_{s q}, \mathbf{M}_{s s}$ & $\Phi_{r i}, \Phi_{q i}, \Phi_{s i}$ \\
\hline $\mathbf{M}_{A}$ & $(4.19),(4.21)$ & $\mathbf{M}_{r q}, \mathbf{M}_{r s}, \mathbf{M}_{s q}, \mathbf{M}_{s s}$ & $\Phi_{r i}, \Phi_{q i}, \Phi_{s i}$ \\
\hline $\mathbf{Q}_{A}$ & $(4.19),(4.22)$ & $\begin{array}{c}\mathbf{M}_{q s}, \mathbf{M}_{s r}, \mathbf{M}_{r q} \\
\mathbf{M}_{q q}, \mathbf{M}_{s s}\end{array}$ & $\Phi_{r i}, \Phi_{q i}, \Phi_{s i}$ \\
\hline \multicolumn{4}{|c|}{ Viscous effect on the hull } \\
\hline $\mathbf{F}_{V}$ & $(4.35),(2.15),(4.36)$ & & \\
\hline $\mathbf{M}_{V}$ & $\begin{array}{l}(4.35),(2.18) \\
(4.37),(4.38)\end{array}$ & $\mathbf{I}_{V 1, i}, \mathbf{I}_{V 2, i}$ & $d S / d \varepsilon, R$ \\
\hline $\mathrm{Q}_{V}$ & $(4.35),(4.40)$ & $\mathbf{I}_{V 1, i}, \mathbf{I}_{V 2, i}$ & $d S / d \varepsilon, R$ \\
\hline \multicolumn{4}{|l|}{ Force acting on fins } \\
\hline $\mathbf{F}_{F}$ & $(4.42),(2.23)$ & & \\
\hline \multirow[t]{2}{*}{$\mathbf{M}_{F}$} & $(4.42),(4.43)$, & $\mathbf{I}_{F i}$ & $\Delta C_{p \alpha}\left(1+\frac{R^{2}}{s^{2}}\right)$ \\
\hline & $(2.25),(4.44)$ & & \\
\hline $\mathbf{Q}_{F}$ & $(4.42),(4.46)$ & $\mathbf{I}_{F i}$ & $\Delta C_{p \alpha}\left(1+\frac{R^{2}}{s^{2}}\right)$ \\
\hline \multicolumn{4}{|c|}{ Force on the hull due to the fins } \\
\hline $\mathbf{F}_{H(F)}$ & $(4.42),(4.47)$ & & \\
\hline $\mathbf{M}_{H(F)}$ & $\begin{array}{l}(4.42),(4.48) \\
(4.49),(4.50)\end{array}$ & $\mathbf{I}_{H(F), i}$ & $\pi R^{2} C_{H(F)}$ \\
\hline $\mathbf{Q}_{H(F)}$ & $(4.42),(4.52)$ & $\mathbf{I}_{H(F), i}$ & $\pi R^{2} C_{H(F)}$ \\
\hline \multicolumn{4}{|l|}{ Axial drag } \\
\hline $\mathbf{F}_{\text {Axial }, H}, \mathbf{F}_{\text {Axial }, F}$ & $(2.28)$ & & \\
\hline $\mathbf{M}_{\text {Axial }, H}, \mathbf{M}_{A x i a l, F}$ & $(4.53),(4.54)$ & $\mathbf{I}_{A S, i}, \boldsymbol{\Phi}_{i}(1 / 4 c)$ & $S$ \\
\hline $\mathbf{Q}_{\text {Axial }, H}, \mathbf{Q}_{A x i a l, F}$ & 0 & & \\
\hline \multicolumn{4}{|c|}{ Force due to control surface deflection } \\
\hline $\mathbf{F}_{\delta}$ & $(2.29),(2.31),(2.32)$ & & \\
\hline $\mathbf{M}_{\delta}$ & $(4.55)$ & $\boldsymbol{\Phi}_{i}\left(\mathbf{r}_{\delta}\right)$ & \\
\hline $\mathrm{Q}_{\delta}$ & $(4.56)$ & $\Phi_{i}\left(\mathbf{r}_{\delta}\right)$ & \\
\hline
\end{tabular}




\subsubsection{Implementation}

A dynamics simulation program was developed in the MATLAB environment to implement the nonlinear dynamics model of a flexible airship according to the following steps. First, the normal mode shapes are calculated to be used as the shape functions. In this work, the eigen problem for the free-free beam vibration is solved numerically using a finite element model with Euler-Bernoulli beam elements. Second, we evaluate the shape function integrals listed in Tabs. 5.1 and 5.2. Since the numerical mode shapes are used as shape functions, the shape function integrals are obtained by numerical quadrature using Simpson's rule. Third, the nonlinear dynamics equations of motion (3.11) are solved numerically in MATLAB using one of the algorithms from the MATLAB ODE suite, ode15s, which is based on the numerical differential formulas [115].

This dynamics simulation program seamlessly integrates the flight dynamics, structural dynamics, aerostatics and aerodynamics of a flexible airship. The elastic deformation is represented by time-dependent generalized coordinates and time-independent shape functions. Accordingly, the effects of flexibility on various forces (inertial, gravitational, control, aerostatic and aerodynamic) are expressed in terms of generalized coordinates, generalized velocities, and shape function integrals. In addition, the simulation program is developed in a modular way. Various force terms are computed in different modules, and the algorithm for each term can be replaced by an alternative computational approach if desired.

\subsubsection{Validation}

Considering the complexity of the dynamics model and the simulation program, several steps were taken to check the validity of the results.

1. The free-free beam mode shape solver is used to perform modal analysis for a 
uniform beam and a double conical beam, because analytical results for natural frequencies and mode shapes are available for these beams $[87,116]$. We find that the numerical mode shapes are nearly identical to the analytical results. In addition, for a free-free Euler-Bernoulli beam, the shape function integrals must satisfy the equalities $\int_{L} \Phi_{i} \bar{m} d x=\int_{L} x \Phi_{i} \bar{m} d x=0$, according to the orthogonality conditions. These integral results are also used to check the numerical mode shape results.

2. The added-mass matrix computation module is based on the procedure discussed in Section 4.2.4. It is used to calculate the added mass and moment of inertia for a rigid ellipsoid $\left(\mathbf{M}_{r r}\right)$ and the generalized added mass $\left(\mathbf{M}_{q q}\right)$ for an elastic ellipsoid, because the corresponding analytical results can be found in the literature $[25,100]$. We find that the added-mass matrix results obtained in the present procedure perfectly match the published analytical results.

3. The simulation program is also validated by checking the energy balance of the system. The kinetic energy and the elastic potential energy of the system (the airship and the surrounding fluid) are obtained as

$$
\begin{aligned}
\mathcal{T}_{\text {all }} & =\frac{1}{2}\left[\boldsymbol{v}^{\mathrm{T}}, \dot{\mathbf{q}}^{\mathrm{T}}\right] \mathbf{M}_{\text {sys }}\left[\boldsymbol{v}^{\mathrm{T}}, \dot{\mathbf{q}}^{\mathrm{T}}\right]^{\mathrm{T}}+\frac{1}{2}\left[\boldsymbol{v}^{\mathrm{T}}, \dot{\mathbf{q}}^{\mathrm{T}}\right] \mathbf{M}_{A T}\left[\boldsymbol{v}^{\mathrm{T}}, \dot{\mathbf{q}}^{\mathrm{T}}\right]^{\mathrm{T}} \\
\mathcal{V} & =\frac{1}{2} \mathbf{q}^{\mathrm{T}} \mathbf{K} \mathbf{q}
\end{aligned}
$$

The work $\mathcal{W}$ is equal to the integral of the power input by the gravity, aerostatic, aerodynamic (excluding the potential-flow terms) and control forces over the elapsed time, i.e.,

$$
\mathcal{W}=\int_{0}^{t}\left(\mathcal{P}_{G}+\mathcal{P}_{A S}+\mathcal{P}_{C}+\mathcal{P}_{V}+\mathcal{P}_{F}+\mathcal{P}_{H(F)}+\mathcal{P}_{\text {Axial }}\right) d t
$$

Recall that the subscripts $G, A S, C, V, F, H(F)$ and Axial denote gravity, aerostatic, control, viscous effect on the hull, force on the fin, force on the hull 
due to the fins, and axial drag respectively. It has been verified that at any step in the simulation, the corresponding energy change of the system $\Delta \mathcal{T}_{\text {all }}+\Delta \mathcal{V}$ is approximately equal to the work of external forces $\mathcal{W}$. The discrepancy between $\Delta \mathcal{T}_{\text {all }}+\Delta \mathcal{V}$ and $\mathcal{W}$ occurs because of the computational error in the numerical integration and diminishes as the integration tolerance used in the numerical integration algorithm is reduced.

\subsection{Simulated Airship}

The solution of the equations of motion for a flexible airship requires its geometry, mass per unit length and bending stiffness distribution. In the numerical simulation, the Skyship-500 non-rigid airship (shown in Figs. 1.3 and 2.12(c)), is used as an example, because its geometric and inertial parameters (presented in Table 2.2) are available in [17]. Recall that for a neutral buoyancy for the Skyship-500, the air density and the altitude are $\rho=1.158 \mathrm{~kg} / \mathrm{m}^{2}$ and $Z_{I}=575 \mathrm{~m}$ respectively, and the operating flight speed is usually below $30 \mathrm{~m} / \mathrm{s}$.

The variation of mass per unit length $\bar{m}$ along the centerline is estimated from a CAD model based on the inertial information from [17] and is plotted in Fig. 5.1. The bending stiffness $E I$ is assumed to be identical in the $y$ and $z$ directions and it can be calculated as

$$
E I=\pi R^{3} E T
$$

where $E$ is the elastic modulus of the hull envelope and $T$ is its thickness. However, the envelope material properties were not provided for the Skyship-500 in [17], and thus the bending stiffness used in the simulation is computed from the material properties of the fabric in [70], in particular, $E T=433,440 \mathrm{~N} / \mathrm{m}$. The latter value was measured by biaxial stress cylinder tests for the envelopes of two aerostats [70]. 


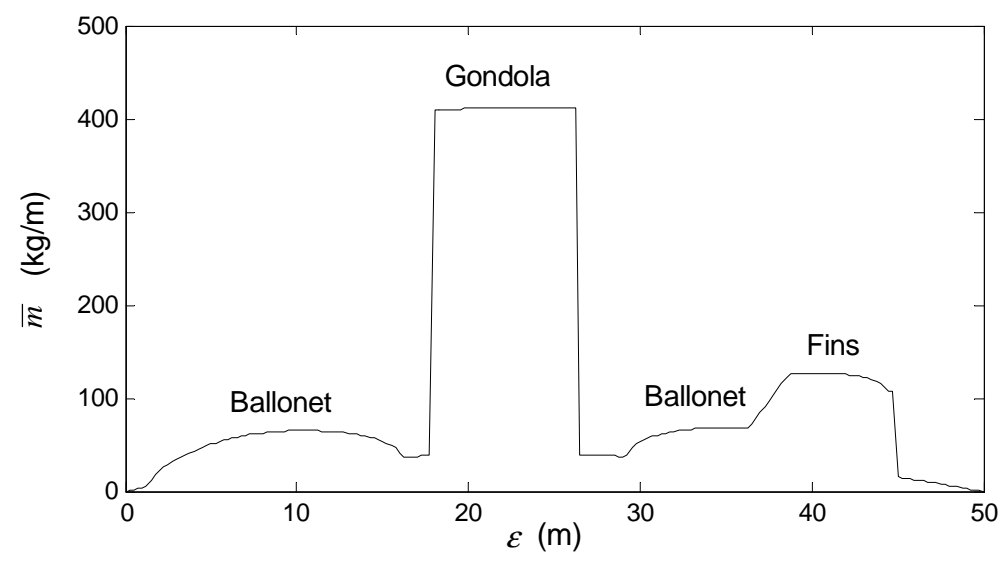

Figure 5.1: Mass per unit length of the Skyship-500

\subsection{Shape Functions and Some Shape Function In- tegrals}

This section presents the shape functions computed by solving for the normal modes of a free-free Euler-Bernoulli beam and selected shape function integrals that reflect the influence of elastic deformation on the inertial and aerodynamic forces.

\subsubsection{Shape functions}

In this work, two shape functions are applied to describe the deflections in each of the $y$ and $z$ directions, i.e., $N=2$ and then the generalized coordinate vector is written as $\mathbf{q}=\left[q_{1}, q_{2}, q_{3}, q_{4}\right]^{\mathrm{T}}$, where $q_{1}$ and $q_{2}$ denote the bending deformation in the $y$ direction while $q_{3}$ and $q_{4}$ denote that in the $z$ direction. Correspondingly, the first and second shape functions can be written as $\boldsymbol{\Phi}_{1}=\left[0, \Phi_{1}, 0\right]^{\mathrm{T}}, \boldsymbol{\Phi}_{2}=\left[0, \Phi_{2}, 0\right]^{\mathrm{T}}$, and the third and fourth ones are $\boldsymbol{\Phi}_{3}=\left[0,0, \Phi_{1}\right]^{\mathrm{T}}, \boldsymbol{\Phi}_{4}=\left[0,0, \Phi_{2}\right]^{\mathrm{T}}$.

The two mode shapes $\Phi_{1}$ and $\Phi_{2}$ are plotted in Fig. 5.2 and the corresponding natural frequencies are: $\omega_{n 1}=22.8 \mathrm{rad} / \mathrm{s}, \omega_{n 2}=64.2 \mathrm{rad} / \mathrm{s}$. We can observe that the deflection in the middle is smaller than those at both ends of the airship, because the bending stiffness in the middle is larger than those at the ends. 

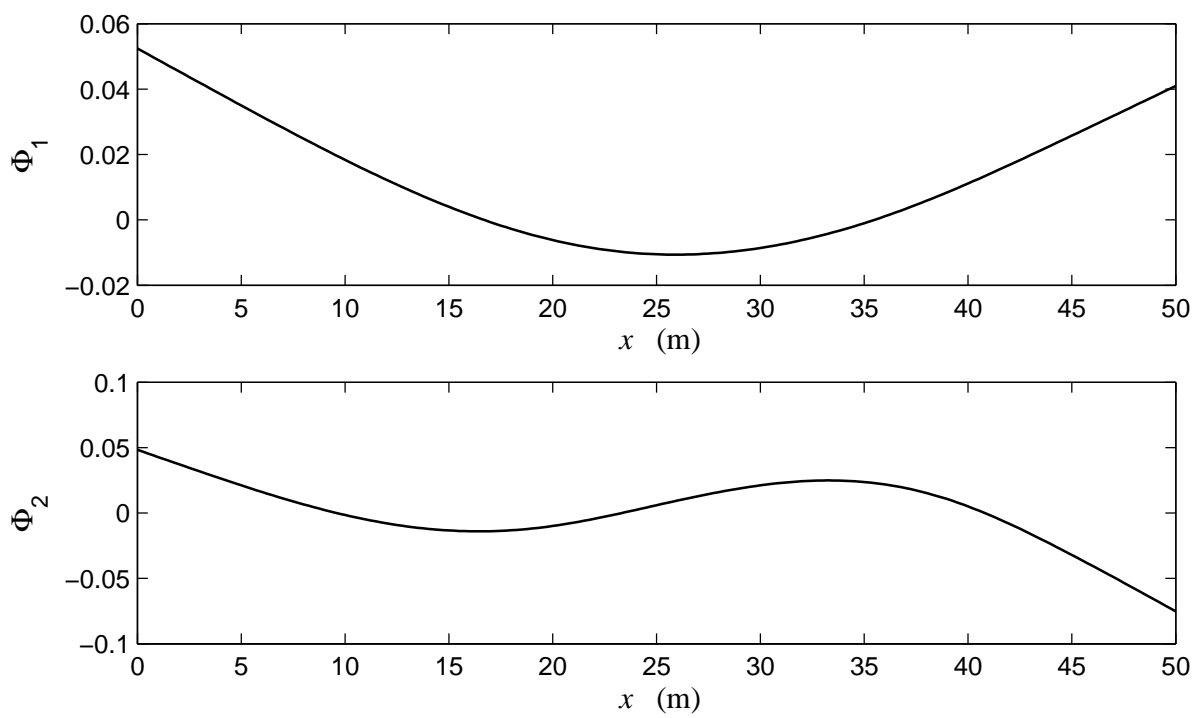

Figure 5.2: The first two normal mode shapes

\subsubsection{Shape function integrals}

Many shape function integrals as listed in Tables 5.1 and 5.2 are included in the equations of motion to incorporate the effects of elastic deformation. Some results of these integrals are given in this section, in order to demonstrate the coupling between flexibility and inertial or aerodynamic forces.

\subsubsection{Effects of deformation on the linear and angular momenta}

According to the second orthogonality condition in Eq. (3.4), we have $\int_{L} \boldsymbol{\Phi}_{i} \bar{m} d x=\mathbf{0}$; thus, the linear momentum matrix defined in Eq. (3.24) is $\mathbf{P}=\mathbf{0}$. In other words, by using the normal modes to express the elastic deformation, the deflection does not affect the position of the C.G., the first moment of inertia computed in Eq. (3.25) and the linear momentum of the flexible airship in Eq. (3.14). 
The angular momentum coefficient matrix defined in Eq. (3.27) is obtained as

$$
\mathbf{H}=\left[\begin{array}{cccc}
178.1 & 50.7 & 0 & 0 \\
0 & 0 & 0 & 0 \\
0 & 0 & 0 & 0
\end{array}\right]
$$

Recall that the $i^{\text {th }}$ column of the matrix $\mathbf{H}$ is defined as $\mathbf{h}_{i}=\int_{L} \mathbf{r}^{\times} \boldsymbol{\Phi}_{i} \bar{m} d x$ and represents the influence of the $i^{\text {th }}$ deflection rate $\dot{q}_{i}$ on the angular momentum of the flexible airship. The non-zero elements in the first row of $\mathbf{H}$ reflect the strong coupling between the first two deflection rates (corresponding to the deflection in the $y$ direction) and the $x$ component of the angular momentum (corresponding to the roll rotation). This coupling results from the fact that the C.G. of the airship is below the centerline. The elements in the second and third rows of $\mathbf{H}$ reduce to zero due to the orthogonality condition between the elastic and rigid-body modes of a free-free Euler-Bernoulli beam as discussed in Eq. (3.4).

\subsubsection{Added-mass matrices}

The influence of the flexibility on the added-mass matrices is now shown for the Skyship-500 airship. First, we investigate the effects of different potential-flow terms on the aerodynamic force and moment, from Eqs. (4.19) to (4.22). For a rigid body, the most important effect is the Munk moment [24], which tends to destabilize the pitch and yaw rotations as discussed in Chapter 2. The contributions of structural flexibility to the external forces and moments are given in the second to fourth lines in Eqs. (4.20) and (4.21). From the magnitudes of the elements in the added-mass matrices $\mathbf{M}_{r q}$, $\mathbf{M}_{r s}, \mathbf{M}_{q q}, \mathbf{M}_{s q}, \mathbf{M}_{s s}$, and the magnitudes of $\mathbf{v}_{0}, \boldsymbol{\omega}, \mathbf{q}$ and $\dot{\mathbf{q}}$, we find that, among the nonlinear flexible potential-flow terms, $-u_{0} \mathbf{M}_{r s 1} \dot{\mathbf{q}}$ has the largest contribution to the aerodynamic force $\mathbf{F}_{A, n o n}$, and $-u_{0} \mathbf{M}_{r s 2} \dot{\mathbf{q}}$ has the largest contribution to the moment $\mathbf{M}_{A, n o n}$. To demonstrate the coupling between the rigid-body motion and deformation 
in these nonlinear terms, the matrices $\mathbf{M}_{r s 1}$ and $\mathbf{M}_{r s 2}$ are listed for the Skyship-500 airship as

$$
\mathbf{M}_{r s 1}=\left[\begin{array}{cccc}
0 & 0 & 0 & 0 \\
-1.2 & -4.3 & 0 & 0 \\
0 & 0 & -1.2 & -4.3
\end{array}\right], \quad \mathbf{M}_{r s 2}=\left[\begin{array}{cccc}
0 & 0 & 0 & 0 \\
0 & 0 & 92.7 & -30.9 \\
-92.7 & 30.9 & 0 & 0
\end{array}\right]
$$

The non-zero elements in the second and third rows in $\mathbf{M}_{r s 1}$ denote the effects of the deflection rate $\dot{\mathbf{q}}$ on the forces in the $y$ and $z$ directions, respectively. Similarly, those non-zero elements in $\mathbf{M}_{r s 2}$ represent the coupling between the deflection rate $\dot{\mathbf{q}}$ and the pitch and yaw moments.

We now investigate the influence of potential-flow aerodynamic force on the vibration of the hull. It has been demonstrated that the generalized added-mass matrix can significantly reduce the natural frequencies of a vehicle moving in a heavy fluid, such as a ship [99-102]. The generalized added-mass matrix $\mathbf{M}_{q q}$ of the Skyship-500 is calculated as

$$
\mathbf{M}_{q q}=\left[\begin{array}{cccc}
0.75 & -0.14 & 0 & 0 \\
-0.14 & 0.96 & 0 & 0 \\
0 & 0 & 0.75 & -0.14 \\
0 & 0 & -0.14 & 0.96
\end{array}\right]
$$

Compared to the elastic generalized mass matrix $\mathbf{M}_{e}$, which is identity from Section 3.3.2, we can see that the diagonal components in $\mathbf{M}_{q q}$ will considerably decrease the natural frequencies of the free vibrations. The new natural frequencies of the Skyship-500 in the air are $\omega_{n 1}=17.2 \mathrm{rad} / \mathrm{s}$ and $\omega_{n 2}=46.0 \mathrm{rad} / \mathrm{s}$, approximately 25-30\% lower than those in vacuum as given in Section 5.3.1. 


\subsection{Time Responses}

We begin by presenting the time responses to simple step-like inputs from the elevator and rudder. Then we design a simple controller for the thruster and control surface to simulate a more complicated path-following maneuver. The response results for a flexible airship are compared to those from the dynamics model for a rigid-body vehicle as described in Chapter 2.

To perform the nonlinear dynamics simulation for a flexible airship, we first define the initial values of the motion states and control input. As an initial condition, the airship is in a steady level flight, that is, we define a steady-state forward speed $\bar{u}_{0}$. The airship experiences some static deformation $\overline{\mathbf{q}}$ due to the gravity and aerostatic forces. All the other steady velocity components, $\bar{w}_{0} \bar{v}_{0}, \overline{\boldsymbol{\omega}}$ and $\dot{\overline{\mathbf{q}}}$ are defined as zero at the initial time $t=0$. Substituting the initial motion states into the equations of motion (3.11) and setting the right hand side of the equations to zero, we can obtain the initial control input $\overline{\mathbf{F}}_{C}, \overline{\mathbf{M}}_{C}$ and $\overline{\mathbf{Q}}_{C}$ needed to maintain the steady level flight condition.

\subsubsection{Case I: Response to elevator input}

In this example, the initial steady level flight speeds are $\bar{u}_{0}=30 \mathrm{~m} / \mathrm{s}$ and $\bar{w}_{0}=0$. This forward speed is a high operating speed for the Skyship-500. The elevator deflection input (positive trailing edge downward) consists of a step input of $10^{\circ}$, as plotted in Fig. 5.3. The time histories of the rigid-body linear and angular velocities in the longitudinal plane $\left(u_{0}, w_{0}, q\right)$ and the corresponding aerodynamic forces and moment $\left(F_{A D, x}, F_{A D, z}, M_{A D, y}\right)$ computed from Eq. (4.1) are displayed in Fig. 5.4. Furthermore, these simulation results are compared to those from a model based on a rigid-body assumption. The time responses of the generalized coordinates and velocities of the third and fourth modes (representing the deformation in the $z$ direction) are drawn in 


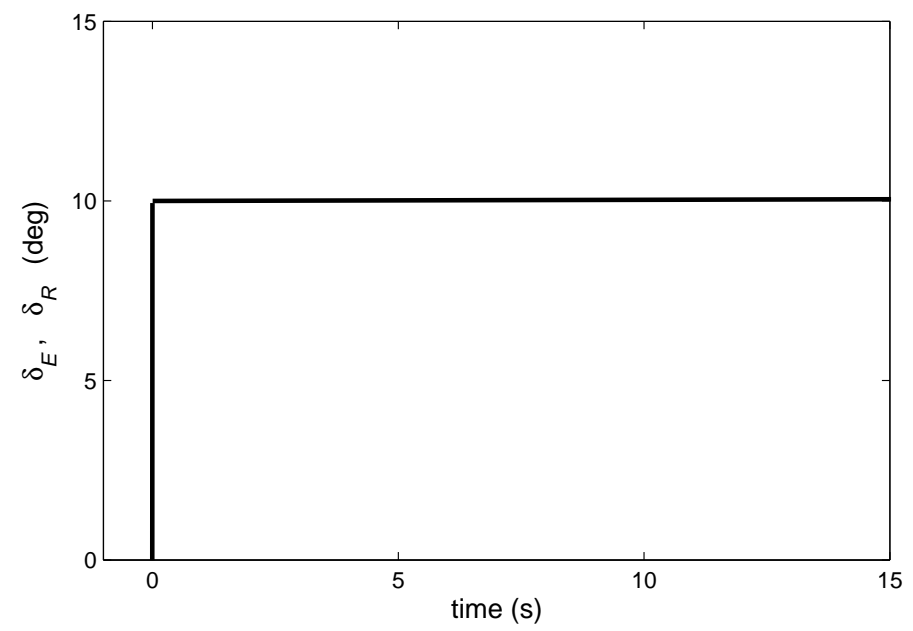

Figure 5.3: Elevator and rudder input in Cases I and II

Fig. 5.5. We can observe small effects of structural flexibility on the rigid-body motion and aerodynamic forces and moment in this maneuver. The oscillation frequencies of $\dot{q}_{3}$ and $\dot{q}_{4}$ is in agreement with the natural frequencies of the airship in air obtained in Section 5.3.2.2. These deflection rates are damped out in about 10 seconds.

\subsubsection{Case II: Response to rudder input}

In the second example, we specify a step rudder input as $\delta_{R}=10^{\circ}$ (positive trailing edge left). A proportional controller is applied to the thrust input so that the airship maintains a constant airspeed. The rigid-body velocities $\left(v_{0}, p, r\right)$ and aerodynamic force and moments $\left(F_{A D, y}, M_{A D, x}, M_{A D, z}\right)$ in the lateral plane are shown in Fig. 5.6. The elastic generalized coordinates and velocities are plotted in Fig. 5.7.

The effects of flexibility on the time histories of rigid-body velocities are minor in this example. Small difference between the flexible and rigid-body dynamics models can be observed in the yaw moment results. The speed in the $y$ direction $v_{0}$ in this case is larger than the speed in the $z$ direction $w_{0}$ in Case I, and causes more aerodynamic damping effects on the elastic motion. Therefore the elastic generalized velocities are damped faster than those in Case I. The oscillation frequencies observed in the $\dot{q}_{1}, \dot{q}_{2}$ 

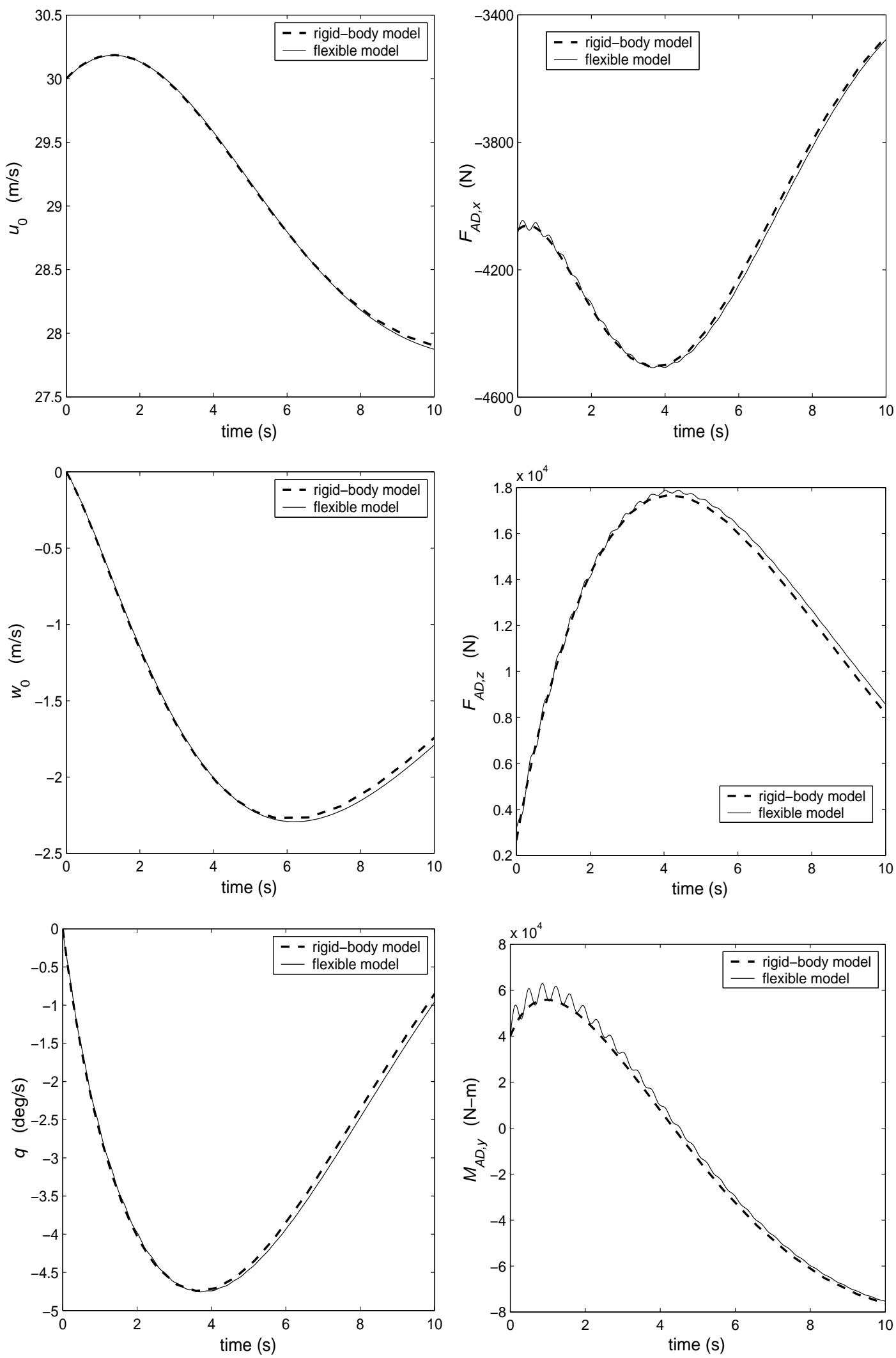

Figure 5.4: Time histories of rigid-body velocities, aerodynamic force and moment, Case I, at $30 \mathrm{~m} / \mathrm{s}$ 

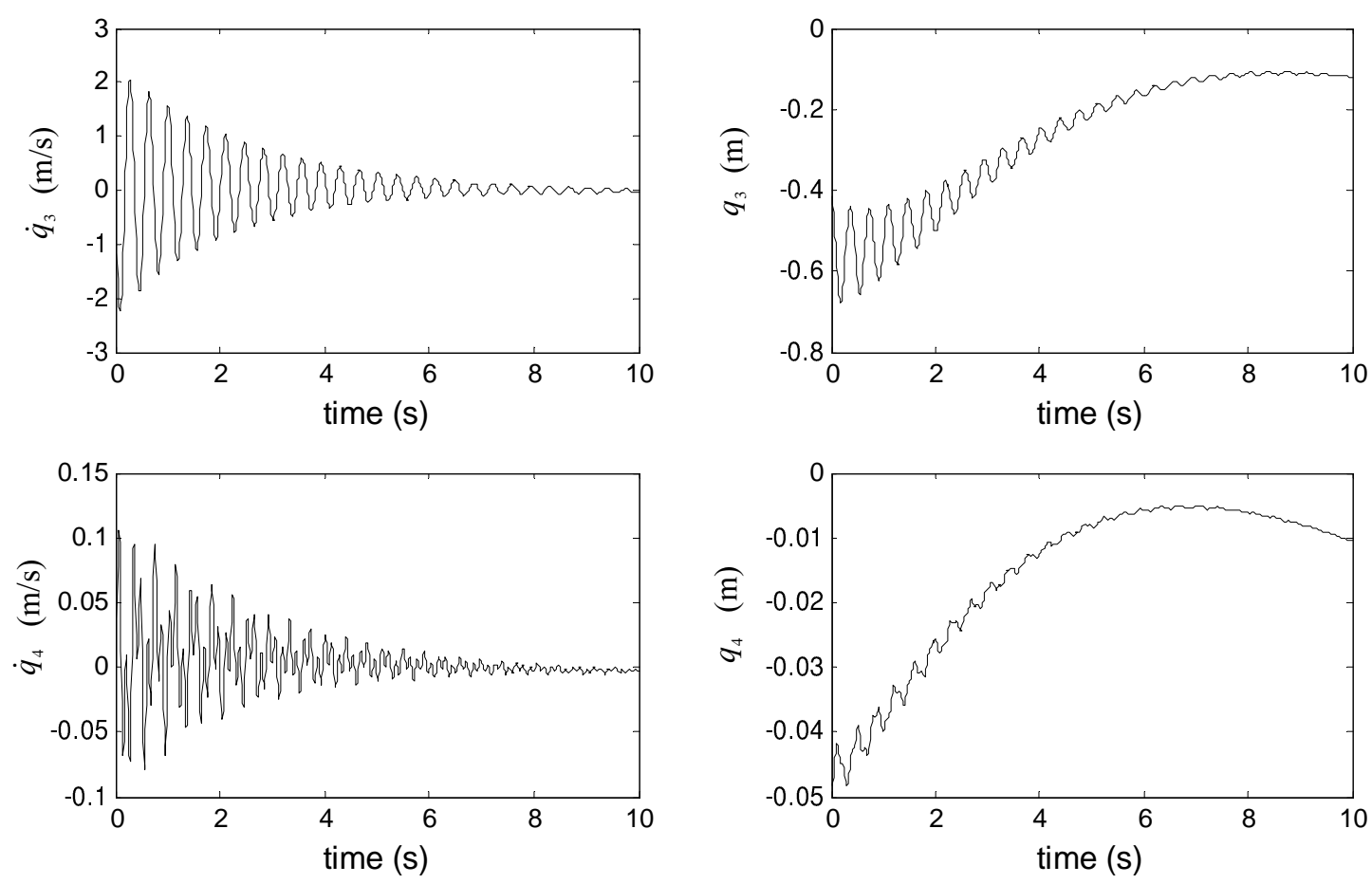

Figure 5.5: Time histories of generalized coordinates and velocities (Case I)

plots are slightly different from those in the $\dot{q}_{3}, \dot{q}_{4}$ plots in Case I. This is due to the coupling between the elastic deformation in the $y$ direction and the roll rotation, as discussed in Section 5.3.2.1.

\subsubsection{Case III: Path-following flights}

In this subsection, we investigate simulation examples where the airship performs a path-following flight in a horizontal plane at two constant forward speeds. The airship is desired to follow a " $\infty "$-shaped path as plotted in Fig. 5.8 (top view) with a radius $\tilde{R}=220 \mathrm{~m}$. The corresponding desired yaw angle $\tilde{\psi}$ and angular velocity $\tilde{r}=\dot{\tilde{\psi}}$ are drawn in Fig. 5.9.

In order to complete this flight task, a simple automatic control system is designed to control the thruster, the elevator and the rudder, discussed as follows.

- Proportional control is applied for the throttle crank input to maintain the 

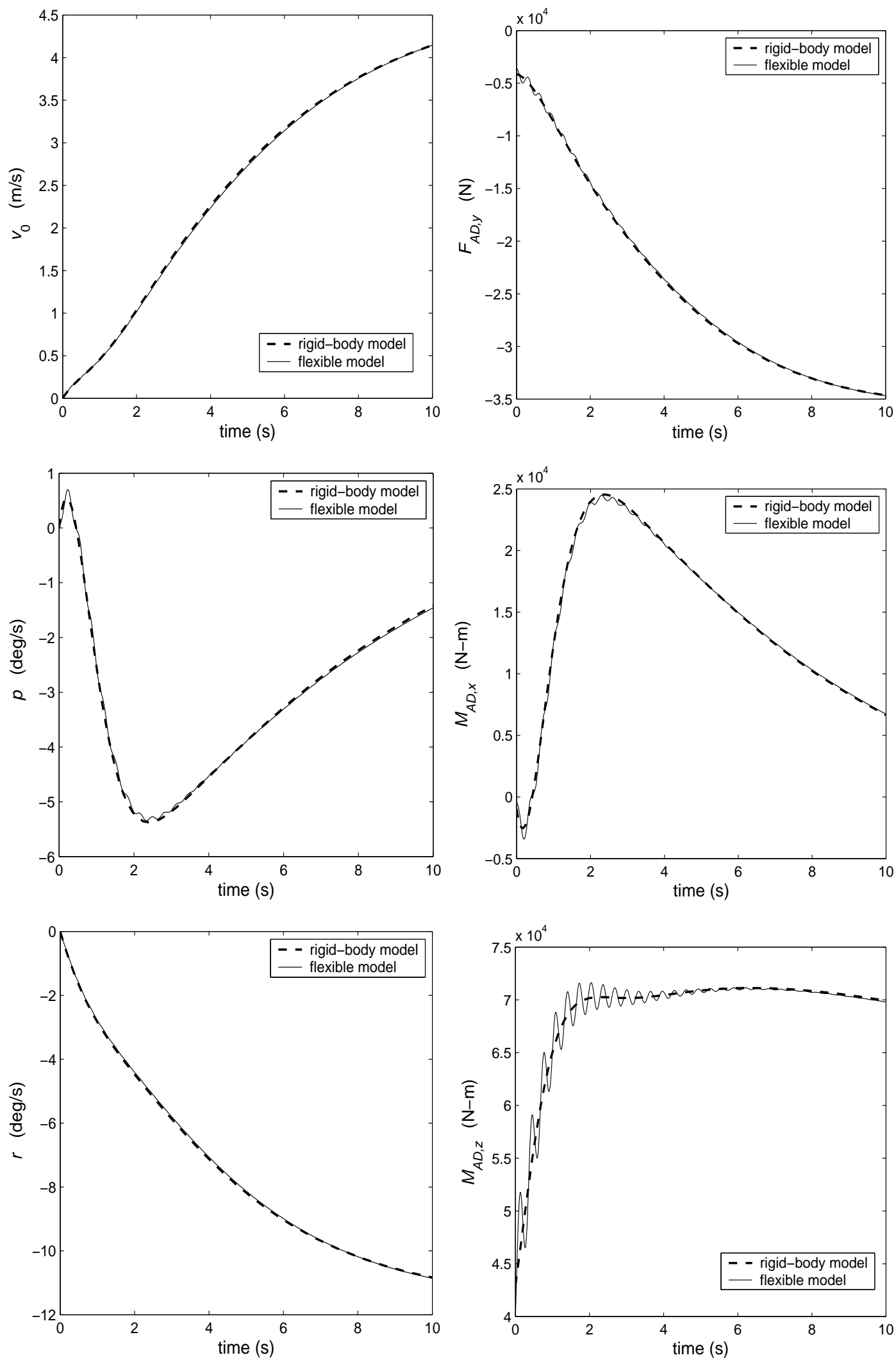

Figure 5.6: Time histories of rigid-body velocities, aerodynamic force and moment, Case II, at $30 \mathrm{~m} / \mathrm{s}$ 

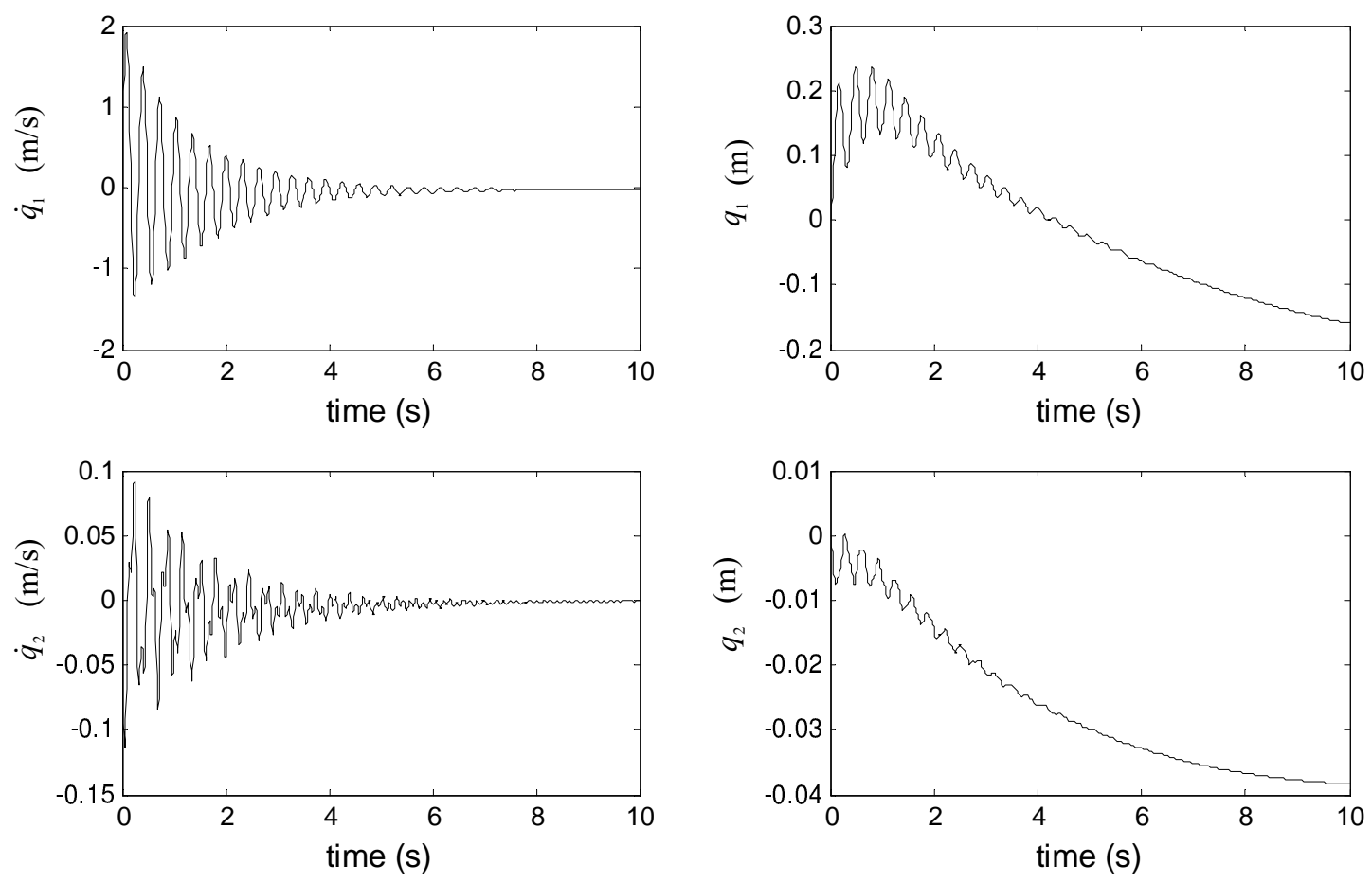

Figure 5.7: Time histories of generalized coordinates and velocities (Case II)

desired forward speed $\tilde{u}_{0}$ as

$$
\delta_{T}=K_{T}\left(\tilde{u}_{0}-u_{0}\right)
$$

- Proportional-integral control is employed for the elevator deflection to maintain the desired velocity $\tilde{w}_{0}$ in the $z$ direction, i.e.,

$$
\delta_{E}=K_{E 1}(\tilde{Z}-Z)+K_{E 2}\left(\tilde{w}_{0}-w_{0}\right)
$$

where $\tilde{Z}$ is the desired altitude in the inertial frame. In this case, $\tilde{Z}=575 \mathrm{~m}$ and $\tilde{w}_{0}=0$.

- Proportional-integral control is used for the rudder deflection to maintain the desired angular velocity $\tilde{r}$ as

$$
\delta_{R}=K_{R 1}(\tilde{\psi}-\psi)+K_{R 2}(\tilde{r}-r)
$$




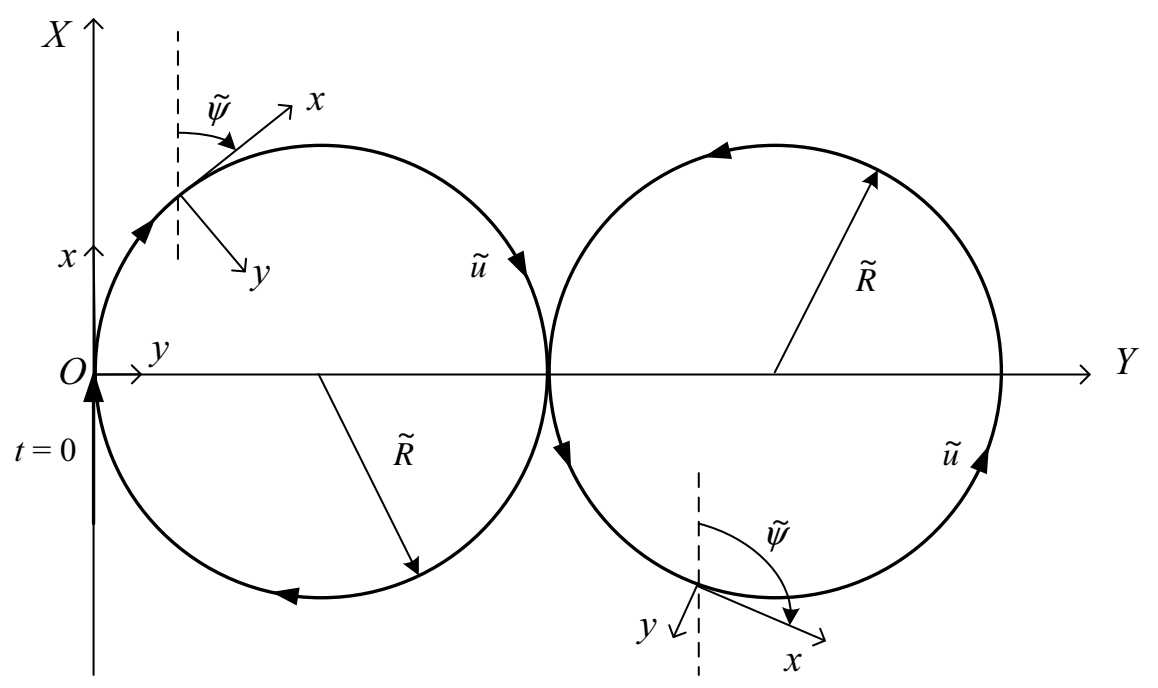

Figure 5.8: Desired path in Case III
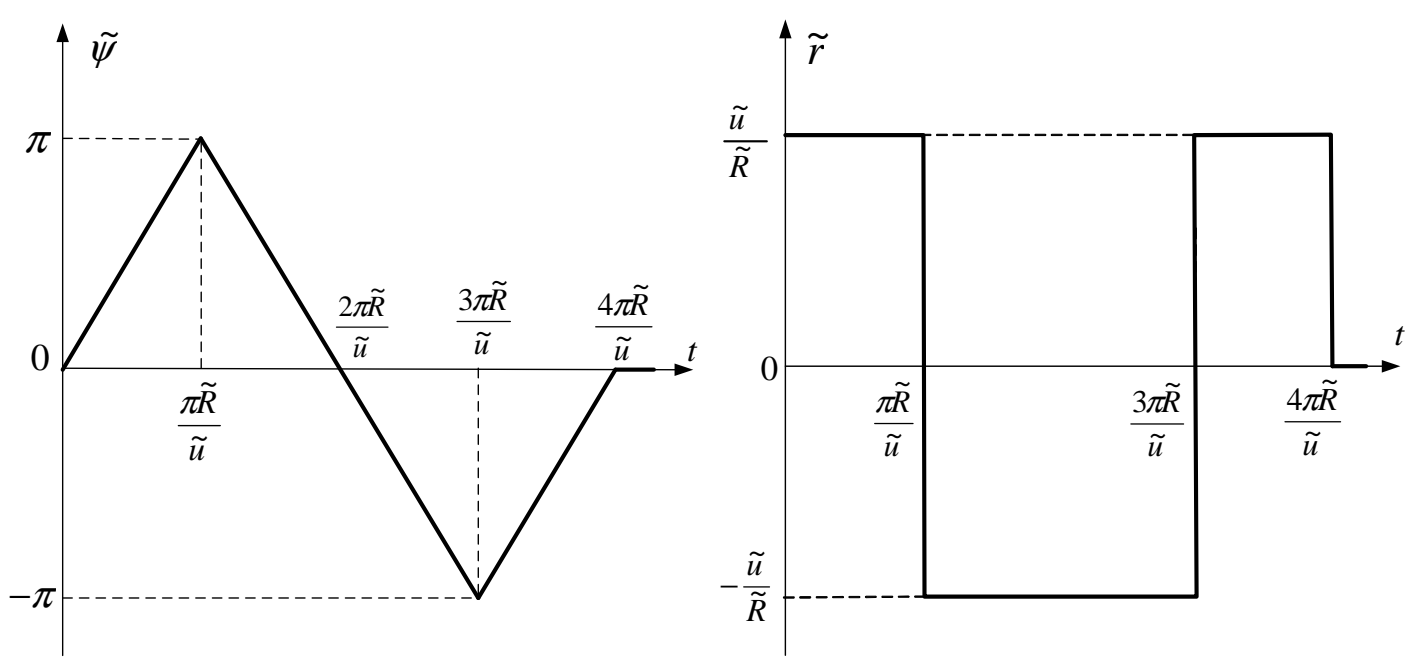

Figure 5.9: Desired yaw angle and yaw rate in Case III

For a real airship, the forward speed $u_{0}$, the velocity in the $z$ direction $w_{0}$, the altitude $Z$, the angular velocity $r$ and the yaw angle $\psi$ are measured by sensors; in the numerical simulation, these motion states are computed in the course of the dynamics simulation. The control gains $K_{T}, K_{E 1}, K_{E 2}, K_{R 1}, K_{R 2}$ are obtained by trial and error and they should be chosen according to the forward speed, for example, the values used at $15 \mathrm{~m} / \mathrm{s}$ and $30 \mathrm{~m} / \mathrm{s}$ for the Skyship-500 are listed in Table 5.3.

The responses of the Skyship-500 at two desired forward speeds $(15 \mathrm{~m} / \mathrm{s}$ and $30 \mathrm{~m} / \mathrm{s}$ ) are obtained by the simulation program. The simulation results of rigid- 
Table 5.3: Control parameters at different speeds

\begin{tabular}{cccccc}
\hline Forward speed $(\mathrm{m} / \mathrm{s})$ & $K_{T}$ & $K_{E 1}$ & $K_{E 2}$ & $K_{R 1}$ & $K_{R 2}$ \\
\hline 15 & -0.044 & 0.65 & -8 & -50 & -150 \\
30 & -0.044 & 0.03 & -2 & -60 & -120 \\
\hline
\end{tabular}

body velocities are plotted in Figs. 5.10 and 5.11 for the airship at $15 \mathrm{~m} / \mathrm{s}$ and $30 \mathrm{~m} / \mathrm{s}$, respectively. The flight path in the $O X Y$ plane and the elevator and rudder inputs are displayed in Fig. 5.12. The higher speed with the above automatic control system leads to larger position errors in the flight path than at the lower speed. The effects of deformation are not important for the rigid-body motion and the control inputs at $15 \mathrm{~m} / \mathrm{s}$; small differences in the elevator input can be observed between the results from flexible and rigid-body models at $30 \mathrm{~m} / \mathrm{s}$. These simulation examples show that the dynamics simulation program can serve as an evaluation tool for automatic control system design.

\subsection{Frequency Response}

A linear dynamics model is now formulated to investigate the frequency responses to control inputs. The linearization procedure employed is similar to that described in Section 2.4, i.e., the state and control matrices are evaluated numerically by using finite difference of the nonlinear equations of motion. For an elastic airship, the dynamics model is represented by $8+4 N$ nonlinear ordinary differential equations. Correspondingly, a state vector $\mathbf{X}$ with $8+4 N$ state variables is defined as follows

$$
\mathbf{X}=\left[u_{0}, w_{0}, q, \theta, \dot{q}_{N+1}, q_{N+1}, \ldots, \dot{q}_{2 N}, q_{2 N}, v_{0}, p, r, \phi, \dot{q}_{1}, q_{1}, \ldots, \dot{q}_{N}, q_{N}\right]^{\mathrm{T}}
$$

The first $4+2 N$ states relate to the longitudinal (rigid-body and elastic) motion and the last $4+2 N$ states relate to the lateral motion. 

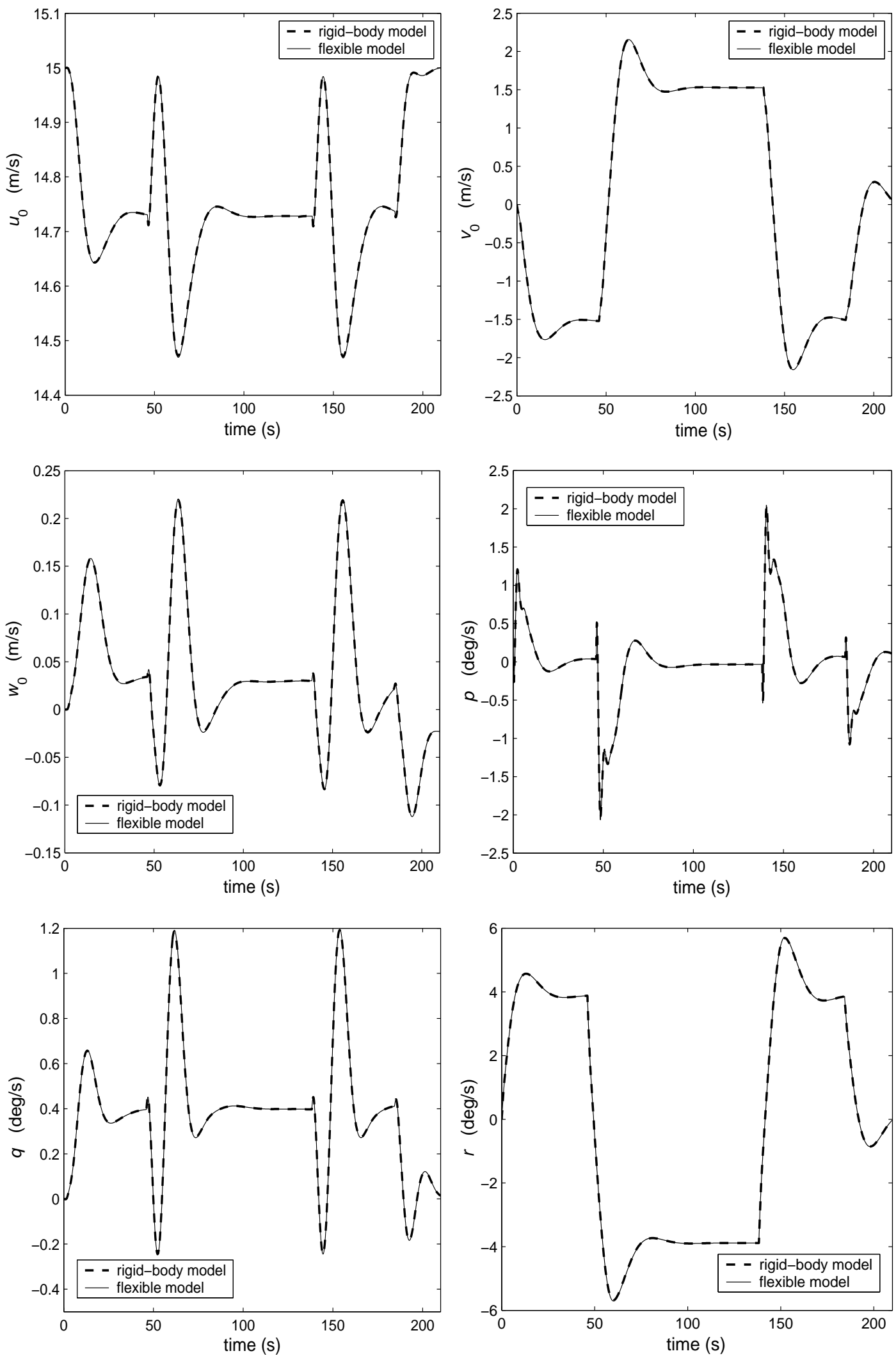

Figure 5.10: Time histories of rigid-body velocities, Case III, at $15 \mathrm{~m} / \mathrm{s}$ 

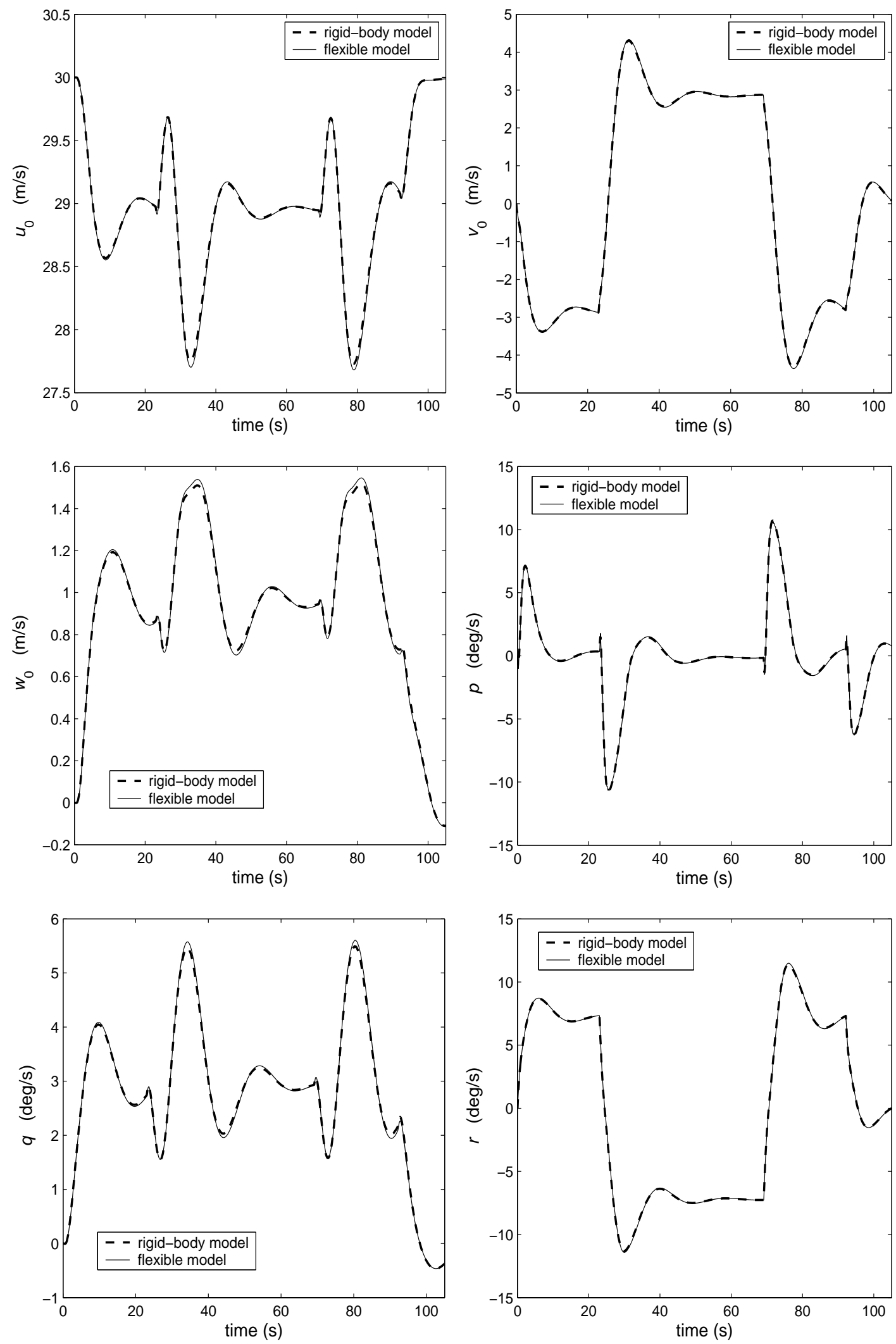

Figure 5.11: Time histories of rigid-body velocities, Case III, at $30 \mathrm{~m} / \mathrm{s}$ 


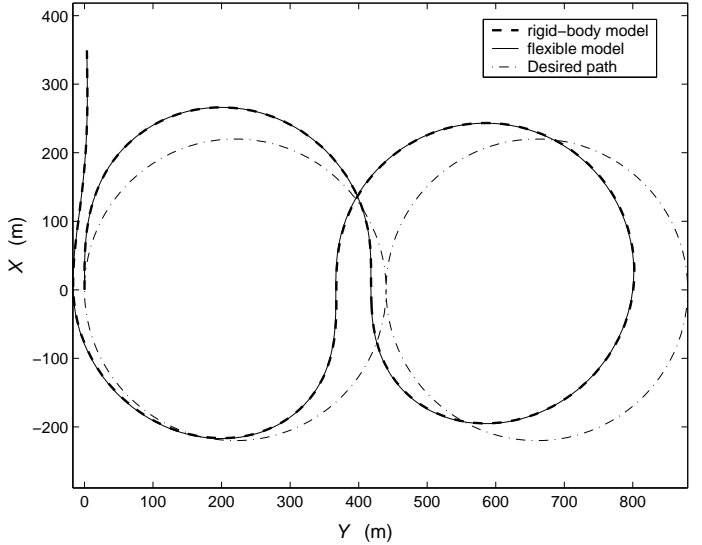

(a) Flight path at $15 \mathrm{~m} / \mathrm{s}$

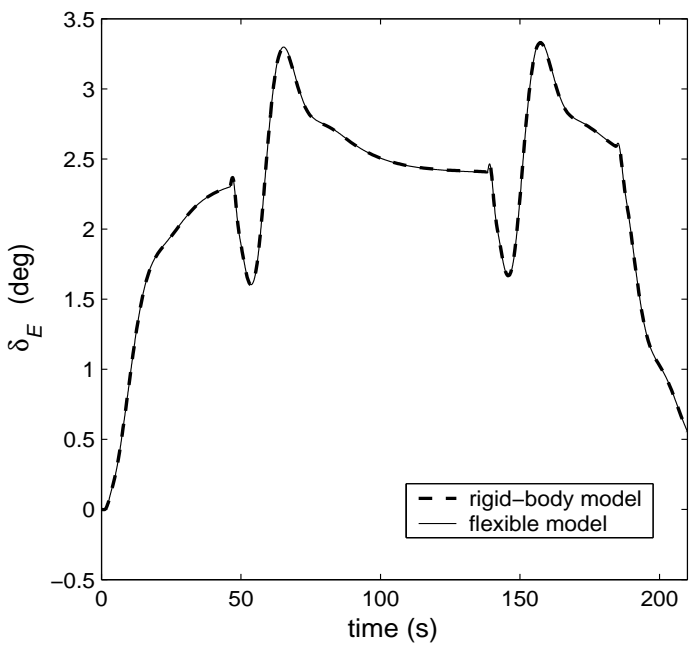

(c) Elevator deflection at $15 \mathrm{~m} / \mathrm{s}$

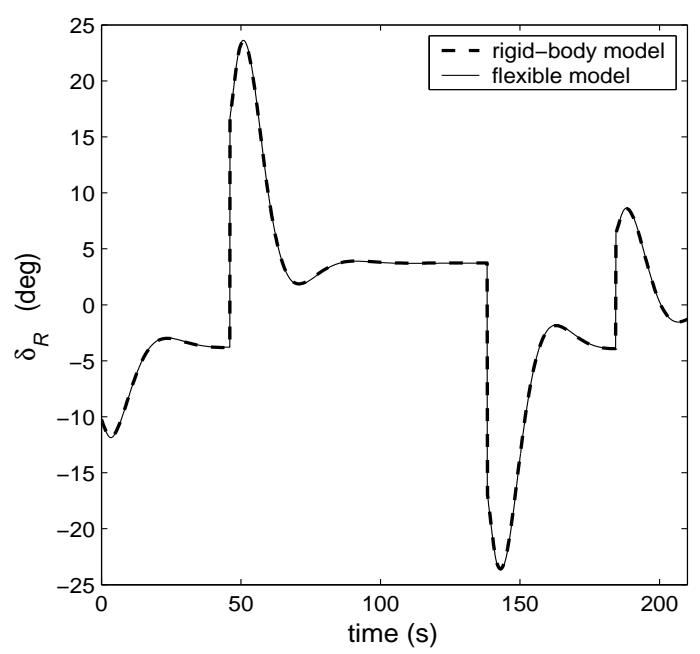

(e) Rudder deflection at $15 \mathrm{~m} / \mathrm{s}$

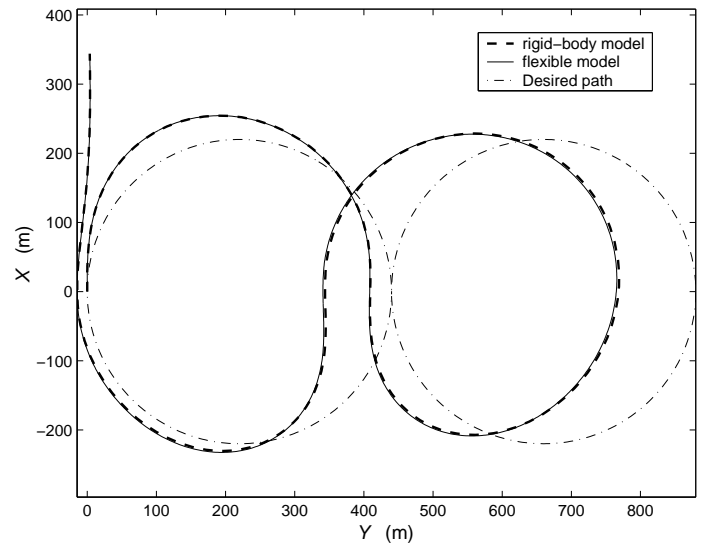

(b) Flight path at $30 \mathrm{~m} / \mathrm{s}$

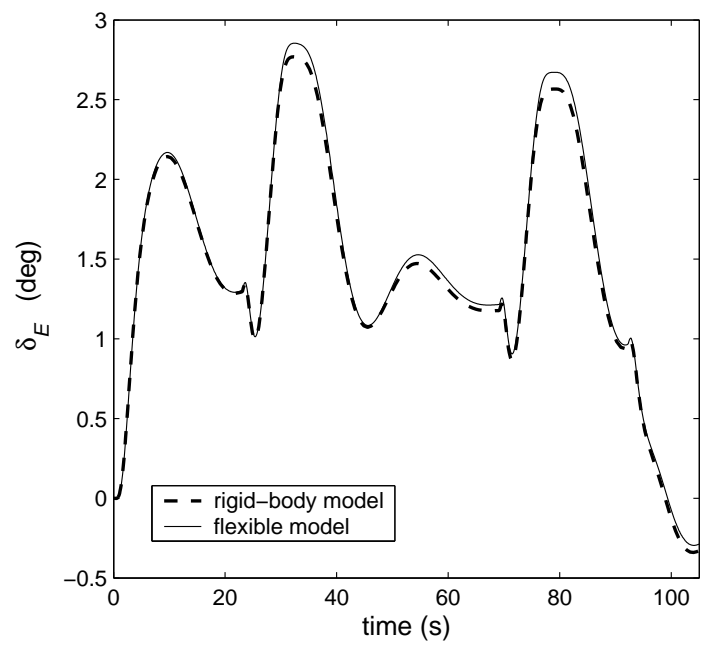

(d) Elevator deflection at $30 \mathrm{~m} / \mathrm{s}$

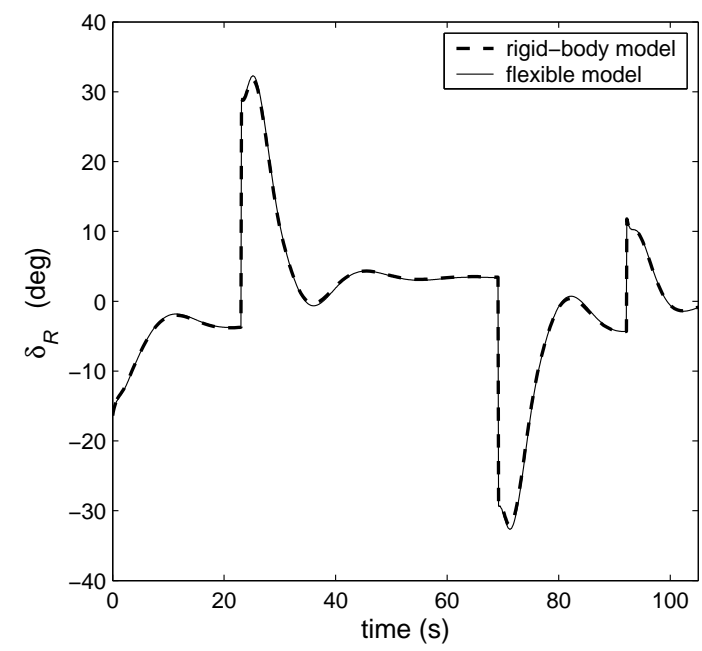

(f) Rudder deflection at $30 \mathrm{~m} / \mathrm{s}$

Figure 5.12: Flight path and control input, Case III 
Once the state and control matrices are obtained, the responses in the frequency domain can be computed. The frequency responses of pitch and yaw rates due to elevator and rudder inputs at 15 and $30 \mathrm{~m} / \mathrm{s}$ are displayed in Bode plots in Figs. 5.13 and 5.14, respectively. In addition, the response results from the flexible airship model are compared to those obtained from the rigid-body dynamics model.

We can observe that 1 ) the flexible and rigid-body models lead to similar results at low frequencies for both longitudinal and lateral motions. 2) The differences between the rigid-body and flexible models occurs at the natural frequencies of the airship in the air. But even if excited at natural frequencies, there is small amplification of the response due to high damping. 3) The influence of flexibility on the yaw rate $r$ is stronger than that on the pitch rate $q$ at both speeds. 4) The natural frequencies observed in the yaw-rate plots are slightly different from those in the pitch-rate plots, because the elastic deflection in the $y$ direction has strong coupling with the roll rotation, as discussed in Section 5.3.2.1, through the angular momentum coefficient matrix. 

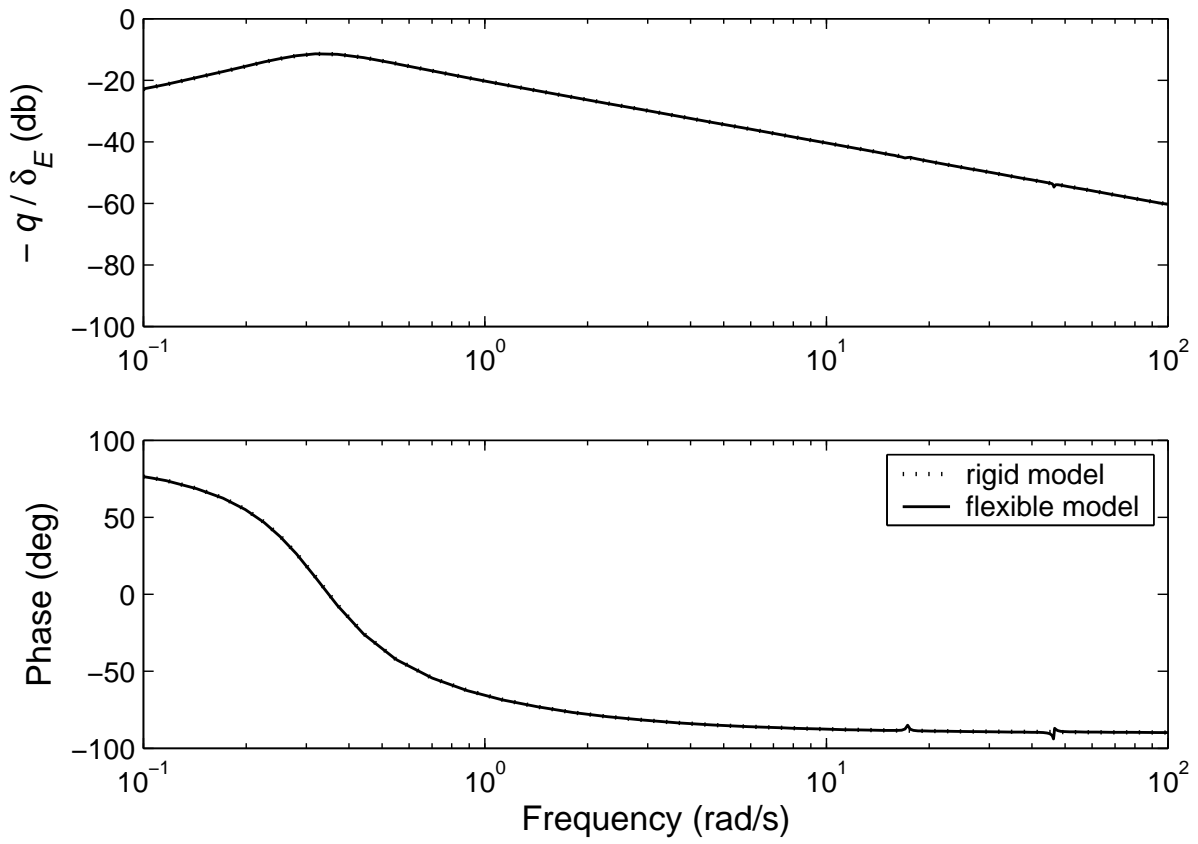

(a) Pitch rate response due to elevator input
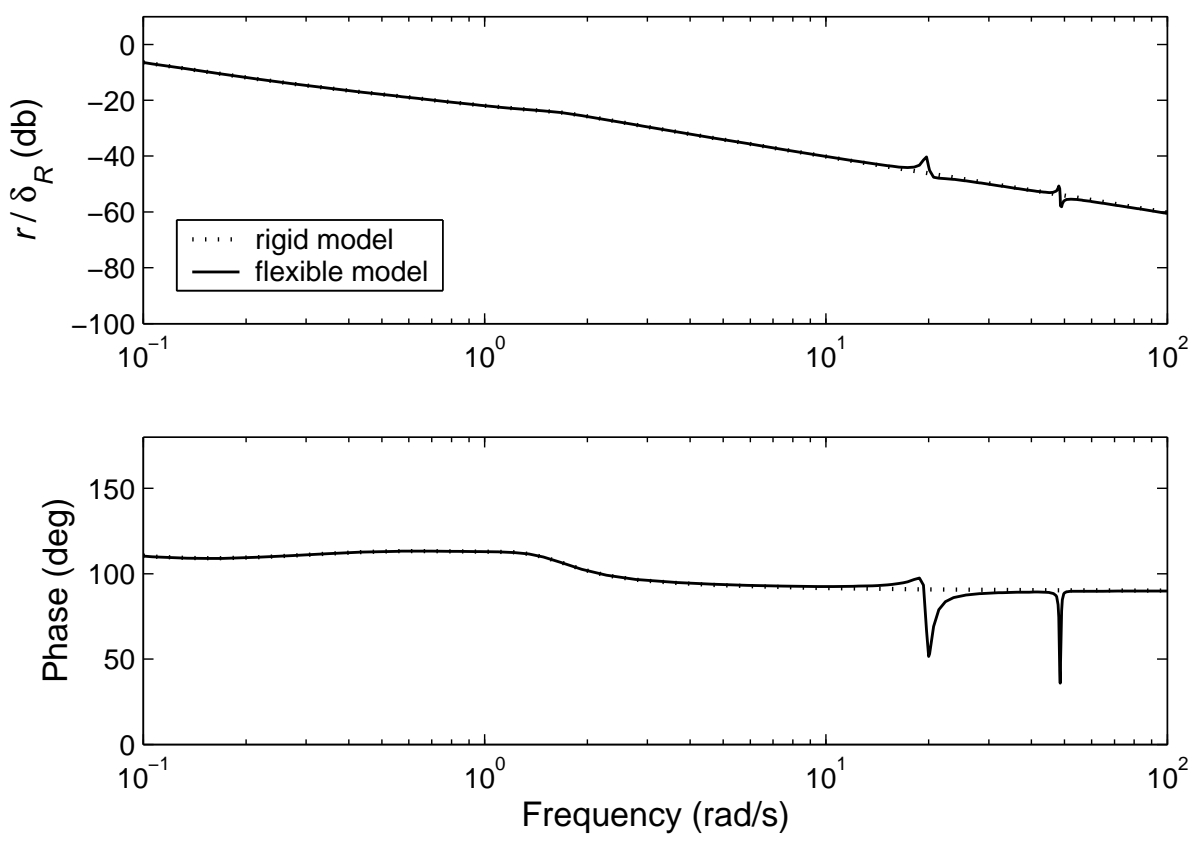

(b) Yaw rate response due to rudder input

Figure 5.13: Control response in the frequency domain at $15 \mathrm{~m} / \mathrm{s}$ 

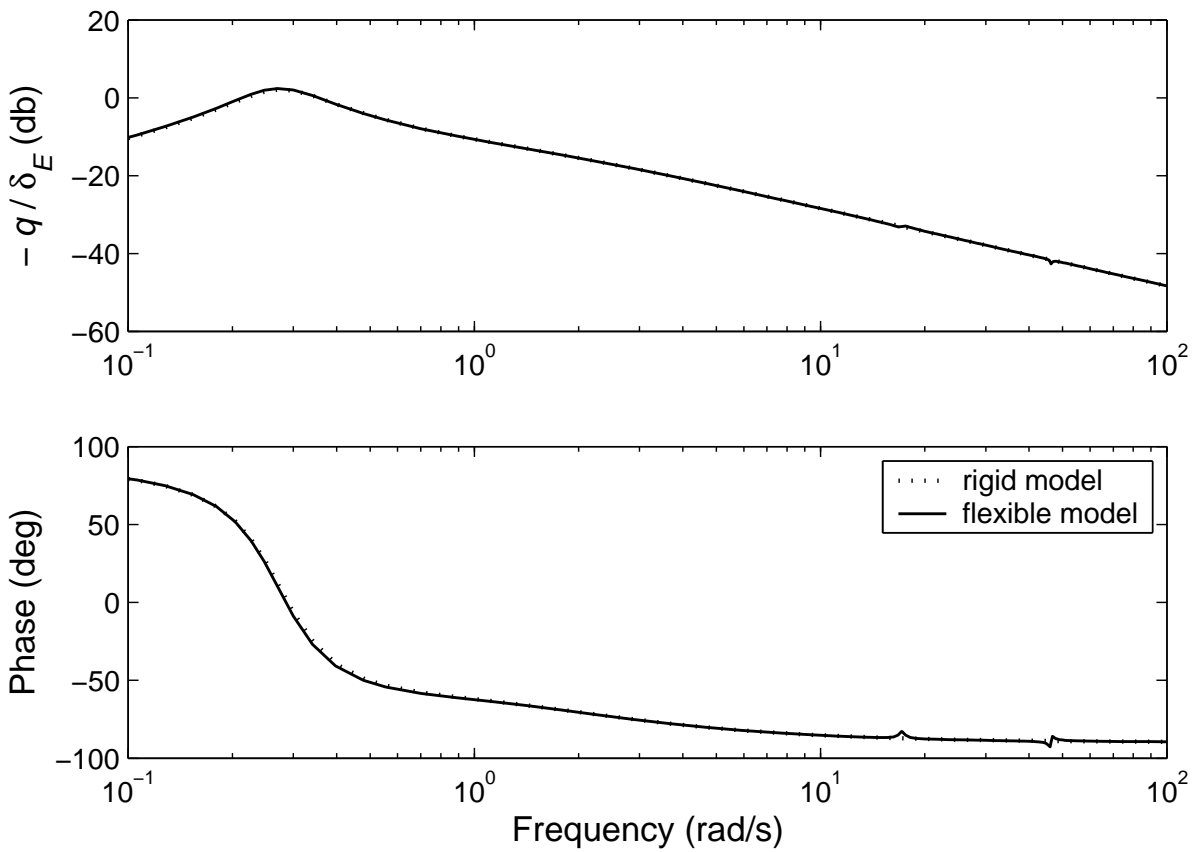

(a) Pitch rate response due to elevator input
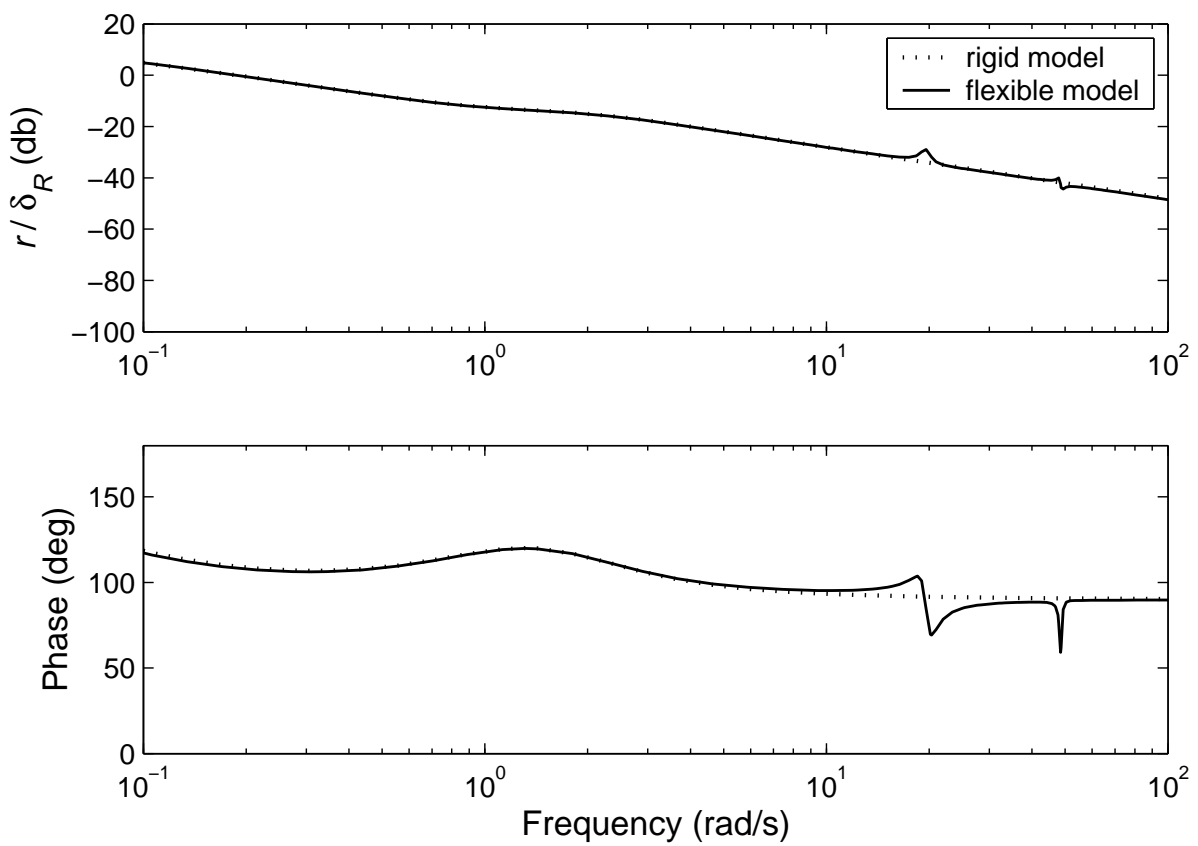

(b) Yaw rate response due to rudder input

Figure 5.14: Control response in the frequency domain at $30 \mathrm{~m} / \mathrm{s}$ 


\section{Chapter 6}

\section{Dynamics Model of Flexible}

\section{Airships: Aeroelastic Stability}

The most important issue in the study of aeroelasticity of conventional HTA aircraft is to determine the conditions at which aeroelastic instability can occur. Although LTA aircraft fly at relatively low speeds, it is still important to investigate the possibility of aeroelastic instability because such instability could have disastrous results. Additional motivation comes from the recently proposed airship designs which make use of very thin films for the vehicle's envelope construction. For example, in [77], a high-altitude airship was designed with the envelope thickness of about $0.048 \mathrm{~mm}$. These envelopes are extremely light in weight, but also provide much lower bending stiffness than the more conventional airship envelopes, because in addition to being very thin, the materials used have lower elastic moduli.

\subsection{Aeroelastic Instability}

In the conventional aeroelasticity of an airplane, the wing is usually modeled as a cantilevered beam mounted at the root. The aerodynamic moment on the wing can 
equivalently lead to a negative aerodynamic torsional stiffness. The static aeroelastic instability (divergence) of a wing is associated with the interaction of aerodynamic force in a steady fluid and the resulting static elastic deformation [87]. Once the airspeed increases to the point where this aerodynamic stiffness exceeds the structural torsional stiffness, divergence occurs. On the other hand, dynamic aeroelastic analysis involves inertial, elastic and aerodynamic forces. The structural dynamics of a wing is represented by a set of linear ordinary differential equations, with the aerodynamic loads linearly expressed in terms of generalized coordinates q, velocities $\dot{\mathbf{q}}$ and accelerations $\ddot{\mathbf{q}}$. The dynamic aeroelastic instability results from the influence of aerodynamic generalized force on the structural inertia, damping and stiffness matrices. For example, the flutter of a wing, the most frequently encountered dynamic aeroelastic instability of an airplane, is caused by an asymmetric aerodynamic stiffness matrix which couples the bending and torsional deformations.

We now investigate the possibility of whether the structural deformation of an airship can lead to instability of the rigid-body or elastic motion. The aeroelastic instability discussed here is different from the divergence or flutter of a cantilevered wing in the following respects. First, the analysis is based on the bending deformation of the hull of an airship and not its fins, because the hull is the largest flexible component of a modern airship, and also because the most important aerodynamic effect, the Munk moment, acts on the hull. Second, the rigid-body translational and rotational motions are included in the aeroelastic analysis here, for example, the inertia matrix considered is not the $2 N \times 2 N$ structural inertia matrix but the $(6+2 N) \times(6+2 N)$ total inertia matrix of the whole flexible airship. Third, we demonstrate that the instability of the airship is due to the coupling between the rigid-body motion and elastic deformation. Specifically, the elastic airship can become unstable because the bending deflection reduces the effectiveness of the fins. In addition, as we have seen in Chapters 3 and 4, the dynamics model of an elastic airship is represented by a set of nonlinear ordinary differential equations and it is intractable to obtain an analyti- 
cal linear model. For the purpose of stability analysis conducted here, the nonlinear model is linearized numerically as discussed previously in Section 5.5.

The aeroelastic unstable phenomenon of an elastic airship is found to be similar to the aeroelastic stability of a slender missile, because it reflects the interaction of the vehicle's aerodynamics, elasticity and flight dynamics. It has been found, in [96], that the instability of a missile was associated with the interaction between its bending, aerodynamic force and rigid-body motion (especially the roll rotation).

\subsubsection{Instability speed}

A linearized dynamic model of the Skyship-500 has already been employed to investigate the influence of deformation on the frequency response. Using this linear model, we investigate the variation of eigenvalues of the state matrix with airship forward speed. Once the real part of any eigenvalue becomes positive, aeroelastic instability occurs. A similar approach was used to obtain the instability condition of a slender missile [96], and it is implemented in the following steps:

1. Define a forward speed range to be evaluated.

2. Use each speed as an equilibrium speed and then formulate the corresponding linear equations of motion.

3. Plot the eigenvalues $\lambda_{1,2}=\sigma \pm \mathrm{j} \omega_{d}$ of the state matrix for each speed. If the real part of any eigenvalue is positive, instability occurs.

The eigenvalues are represented with plots of $\sigma$ and $\omega_{d}$ for the longitudinal and lateral modes respectively in Figs. 6.1 and 6.2. In order to observe the effects of structural flexibility, the eigenvalue results based on the rigid-body dynamics model are revisited in Figs. 6.3 and 6.4, from which we can see that the longitudinal and lateral modes based on the rigid-body assumption are all stable from 0 to $130 \mathrm{~m} / \mathrm{s}$. 
Recall that in the longitudinal plane, the first rigid-body mode is associated with the surge motion caused by aerodynamic axial drag; the second rigid-body mode is due to the coupling between the heave; and pitch motion and the third is a pitch-incidence oscillation mode. In the lateral plane, the first and second lateral rigid-body modes are associated with the coupling sideslip-yaw and yaw-roll motion respectively, and the third is a roll oscillation mode.

From Fig. 6.1, we observe that the aeroelastic instability occurs at approximately $u_{\text {unstable }}=88 \mathrm{~m} / \mathrm{s}$, and we find that the third longitudinal rigid-body mode, related to pitch-incidence oscillation, becomes oscillatory unstable $\left(\sigma>0\right.$ and $\left.\omega_{d}>0\right)$ at this speed. The elements of the eigenvector of the corresponding unstable eigenvalue are dominated by a positive velocity $\Delta w_{0}$ in the $z$ direction (downward) and a negative third generalized coordinate $\Delta q_{3}$ (hull deflecting upward at both ends). That is, the third rigid-body mode becomes a pitch/bending coupling mode for an elastic airship. The cause of the aeroelastic instability is illustrated in Fig. 6.5. The vertical downward velocity $\Delta w_{0}$ leads to a nose-up unstable Munk moment and also generates three upward forces $F_{V, z}, F_{F, z}, F_{H(F), z}$ at the rear of the airship. Recall that the latter three aerodynamic forces result from the hull viscous effect, the force on the fins and the force on the hull due to the fins respectively, and they produce nose-down pitching moments which tend to stabilize the airship. However, the upward deflection at the rear causes a decrease in the local angles of attack, which effectively produces three downward forces $\Delta F_{V, z}, \Delta F_{F, z}, \Delta F_{H(F), z}$. In other words, the deformation reduces the stable aerodynamic effects of viscosity and fin force. As the forward speed increases to $u_{\text {unstable }}=88 \mathrm{~m} / \mathrm{s}, \Delta F_{V, z}, \Delta F_{F, z}, \Delta F_{H(F), z}$ become more significant, so that the viscous effect and the fin force cannot provide enough negative pitch moment to stabilize the airship. The positive imaginary part $\omega_{d}$ of the eigenvalue represents this is an under damped oscillation system at this speed. As the speed increases to $123 \mathrm{~m} / \mathrm{s}$, the deformation further reduces the stable aerodynamic effects, so that the real part of the eigenvalue $\sigma$ becomes more positive and the imaginary part $\omega_{d}$ becomes zero for 

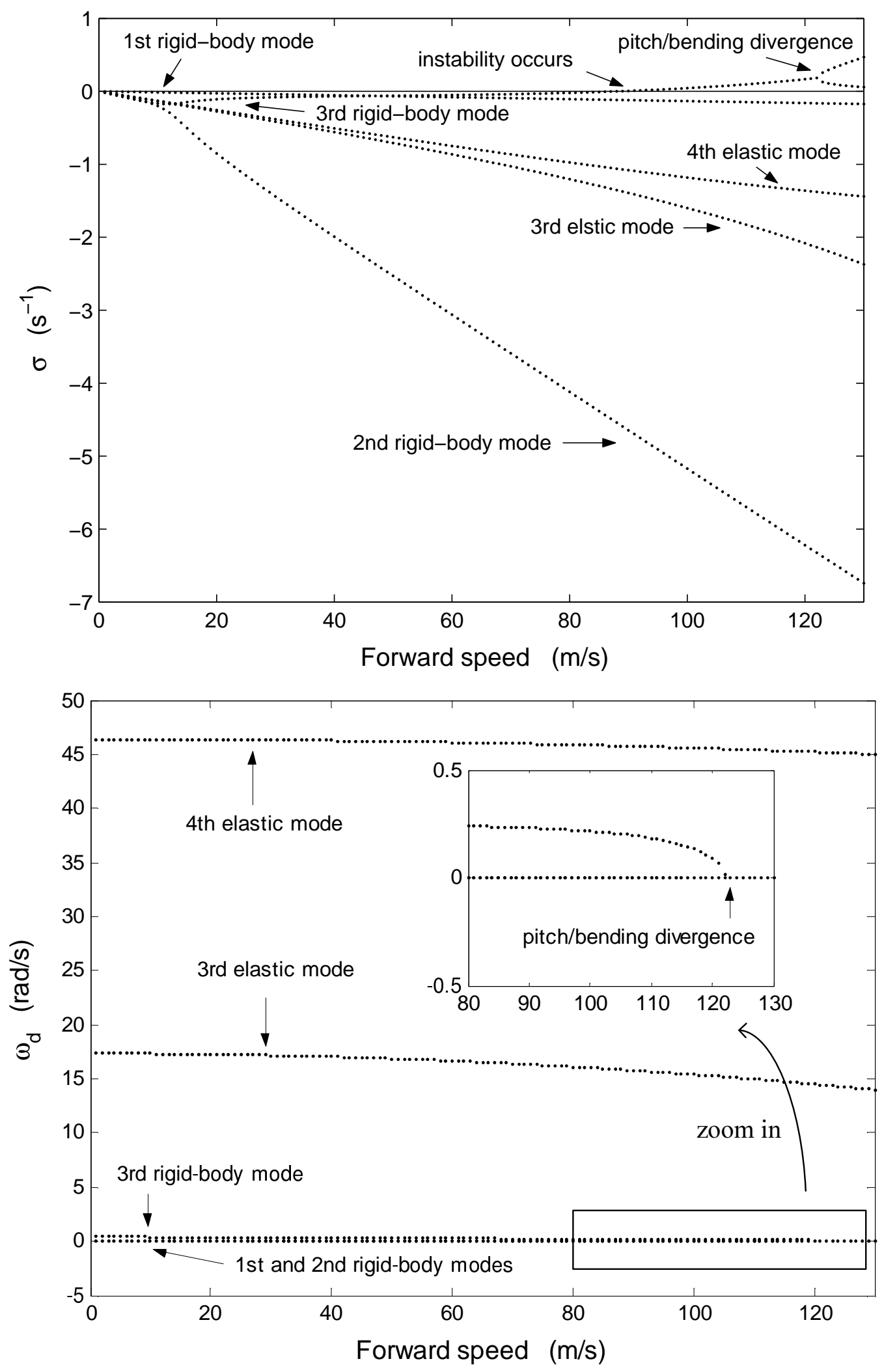

Figure 6.1: Eigenvalues of longitudinal modes from flexible-body dynamics 

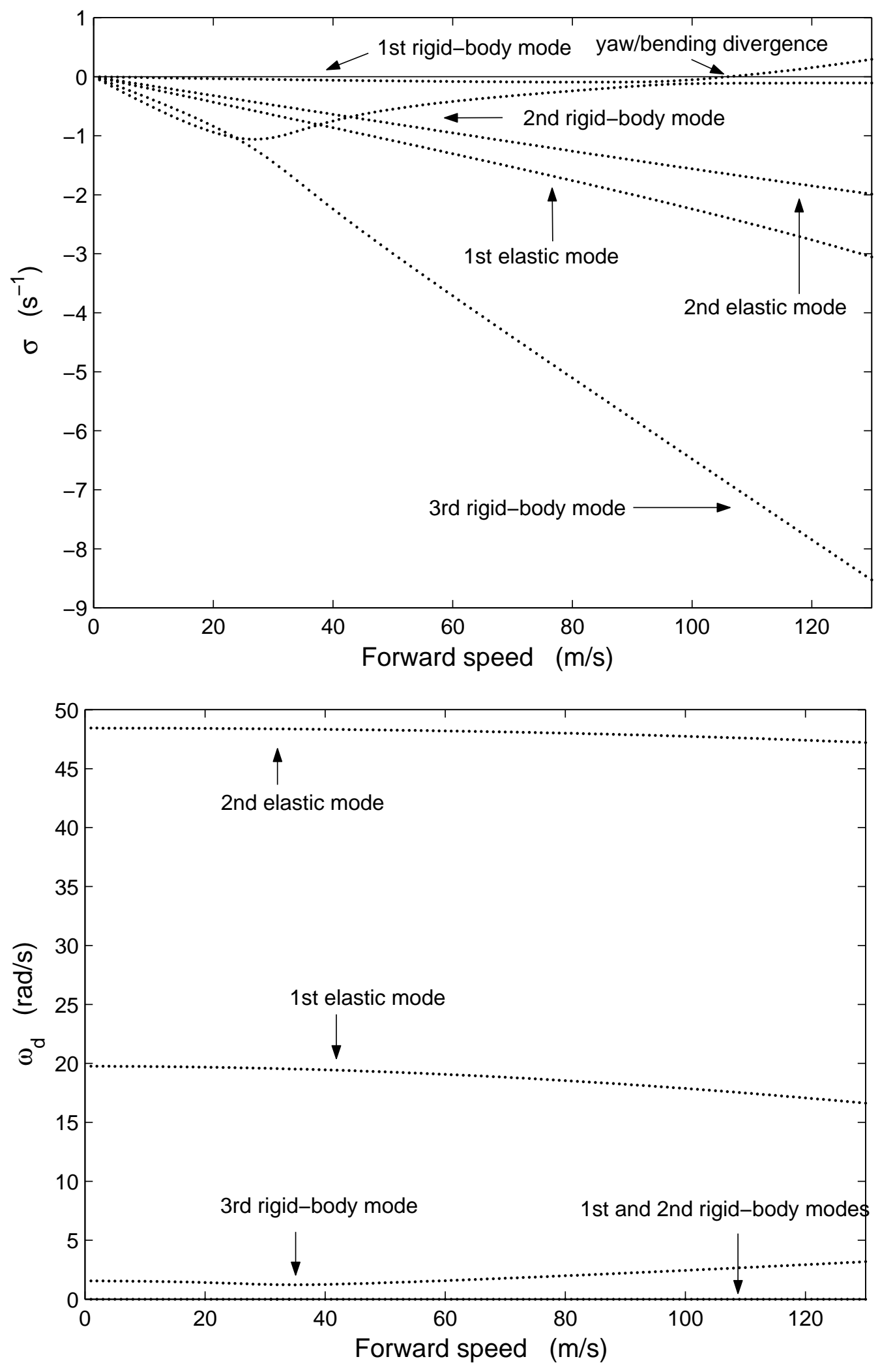

Figure 6.2: Eigenvalues of lateral modes from flexible-body dynamics 

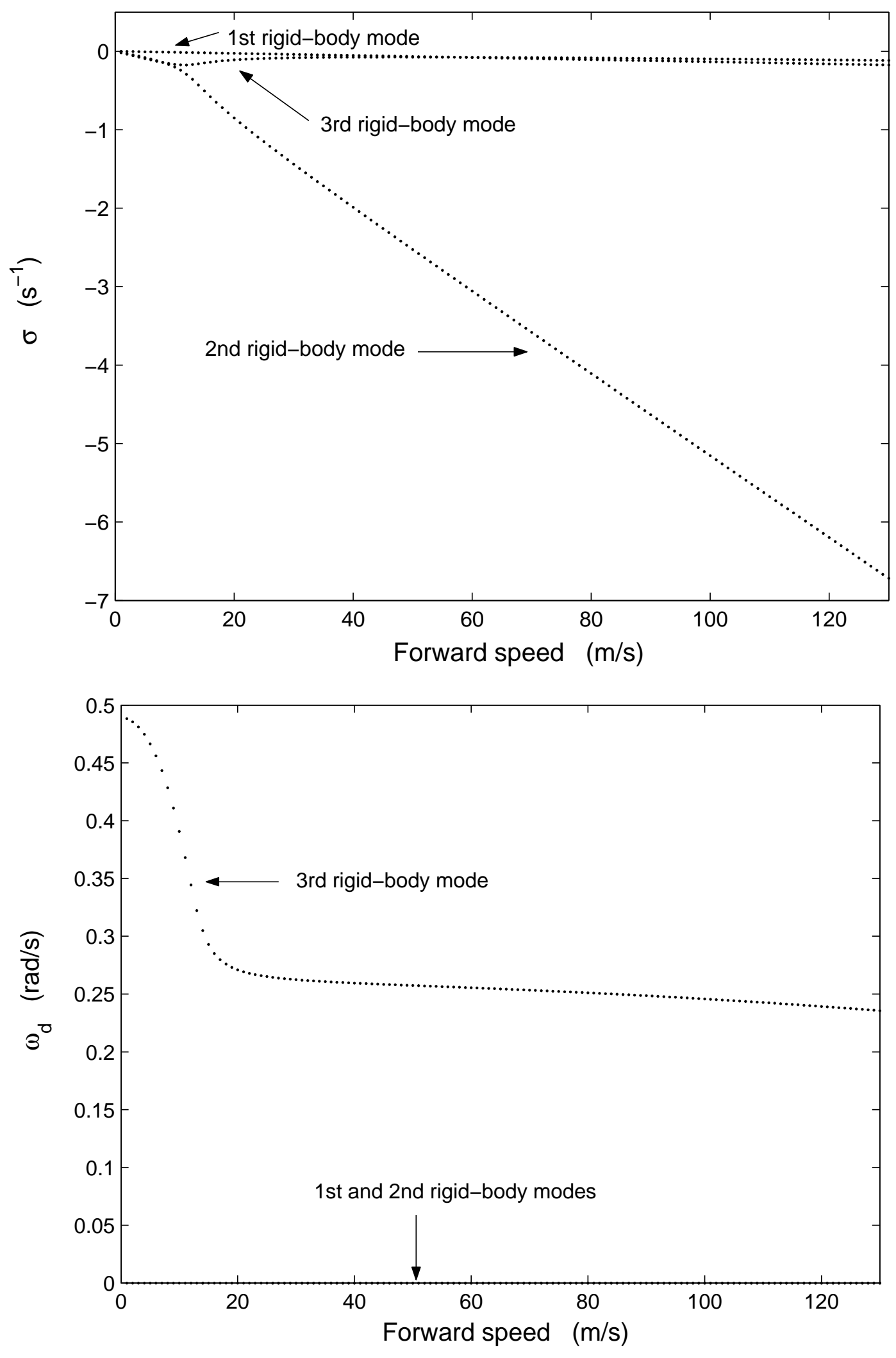

Figure 6.3: Eigenvalues of longitudinal modes from rigid-body dynamics 

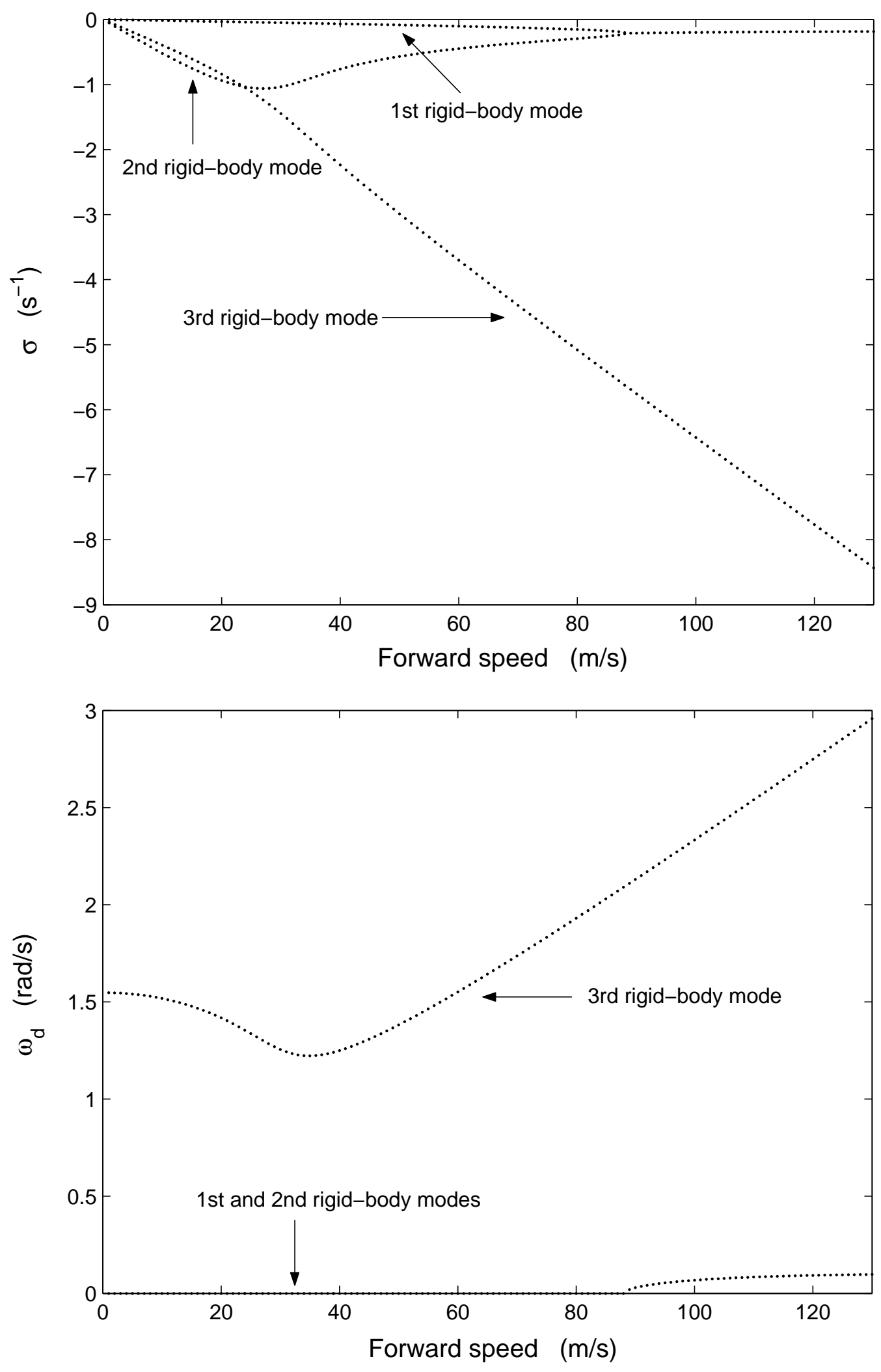

Figure 6.4: Eigenvalues of lateral modes from rigid-body dynamics 


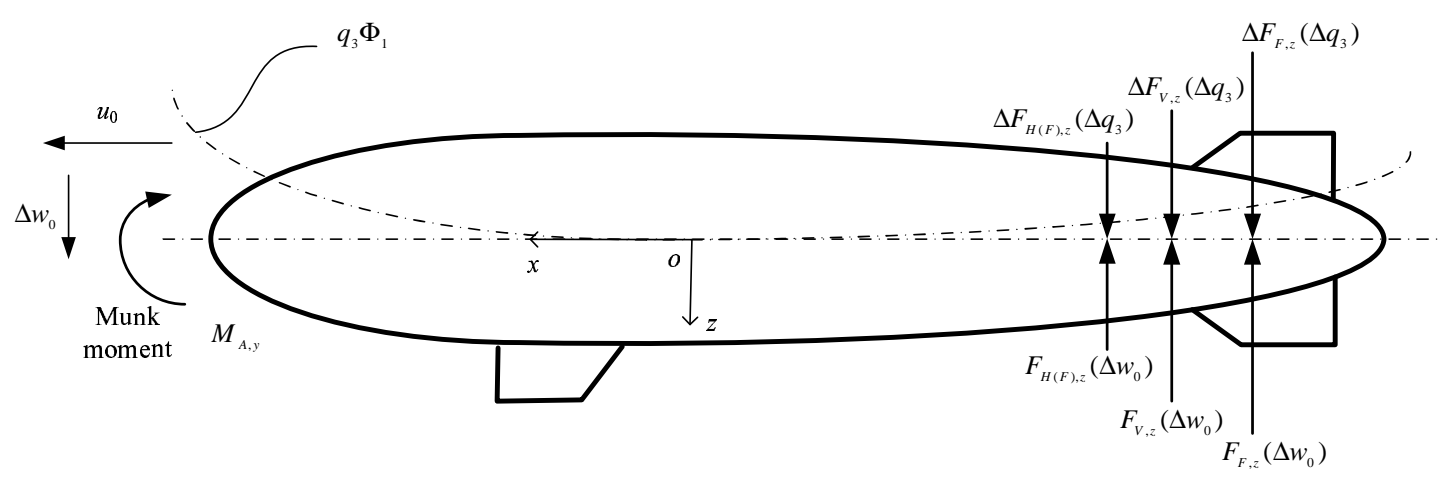

Figure 6.5: Cause of the aeroelastic instability

this mode, representing that pitch/bending divergent instability occurs.

From Fig. 6.2, the first lateral rigid-body mode, related to sideslip-yaw motion, becomes divergent at about $106 \mathrm{~m} / \mathrm{s}$. It can be observed from the eigenvector that this rigid-body mode becomes a yaw/bending coupling mode for an elastic airship. This instability is caused by the yaw Munk moment and by the decrease of the aerodynamic damping effects due to the bending deflection in the lateral plane. Note that there are no oscillatory instabilities that can be observed in the lateral motion, because the deformation has only small influence on the third rigid-body mode that is related to the roll oscillation.

The aeroelastic instability discussed here reflects the interaction between the aerodynamics, deformation and rigid-body motion of the airship. The generation of this instability results from the existence of the unstable aerodynamic Munk moment and from the fact that the deformation reduces the other aerodynamic effects that stabilize the airship. This instability may significantly impact the airship's flight behavior and even cause a catastrophic failure. For the Skyship-500, the operating flight speed is usually less than one half of the lowest aeroelastic instability speed $u_{\text {unstable }}$ determined here. 


\subsubsection{Time response at high speeds}

To validate the previous analysis and to demonstrate that the flexibility can cause instability at the speed $u_{\text {unstable }}$ determined from a linearized model, a nonlinear simulation is performed for the Skyship-500 at high speeds. Similar to the examples discussed in Section 5.4, control inputs are provided so that the airship is in a steady level flight with a forward speed $u_{0}$ at the initial condition. Then, a disturbance to the vertical speed of $\Delta w_{0}=1 \mathrm{~m} / \mathrm{s}$ is given and the transient response is computed by the nonlinear dynamics simulation program.

The time histories of vertical speed $w_{0}$, pitch angle $\theta$ and aerodynamic pitch moment $M_{A D, y}$ are displayed in Figs. 6.6 and 6.7 at forward speeds of $86 \mathrm{~m} / \mathrm{s}, 90 \mathrm{~m} / \mathrm{s}$, $105 \mathrm{~m} / \mathrm{s}$ and $125 \mathrm{~m} / \mathrm{s}$ respectively. The simulated results are compared to those obtained from the rigid-body dynamics model. We can observe the significant impact of the structural flexibility on the rigid-body motion at these speeds. In particular, Fig. 6.6(a) shows that the vertical speed disturbance, the pitch angle and the aerodynamic moment are damped out for the speed of $86 \mathrm{~m} / \mathrm{s}$, while the response in Fig. 6.6(b) shows an oscillatory instability at $90 \mathrm{~m} / \mathrm{s}$. Due to the instability at $105 \mathrm{~m} / \mathrm{s}$, the pitch angle can be as high as $-90^{\circ}$ in about 56 seconds. As the speed increases to $125 \mathrm{~m} / \mathrm{s}$, the longitudinal motion tends to be divergently unstable, and the pitch angle increases be $90^{\circ}$ in only 8 seconds. It is noted that the angle of attack of the airship remains less than $12^{\circ}$ in the above simulation cases at high speeds.

The above simulation results confirm that the stability analysis conducted with the linearized equations of motion can accurately predict the instability condition of the nonlinear model. 


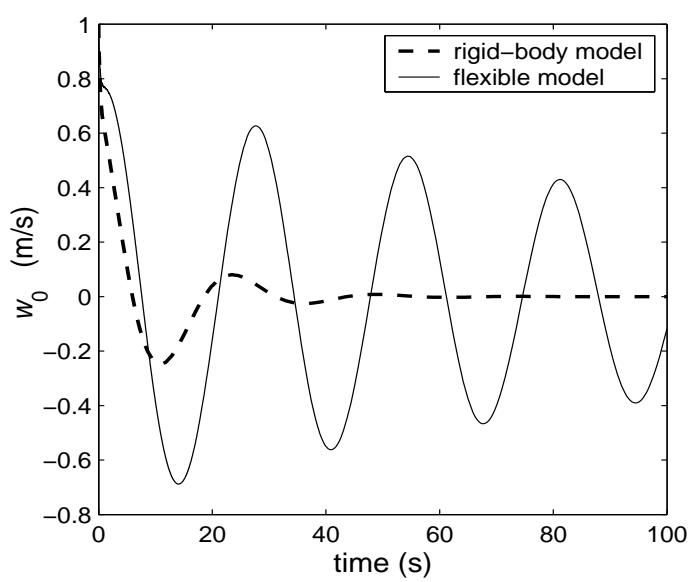

(a) At $86 \mathrm{~m} / \mathrm{s}$

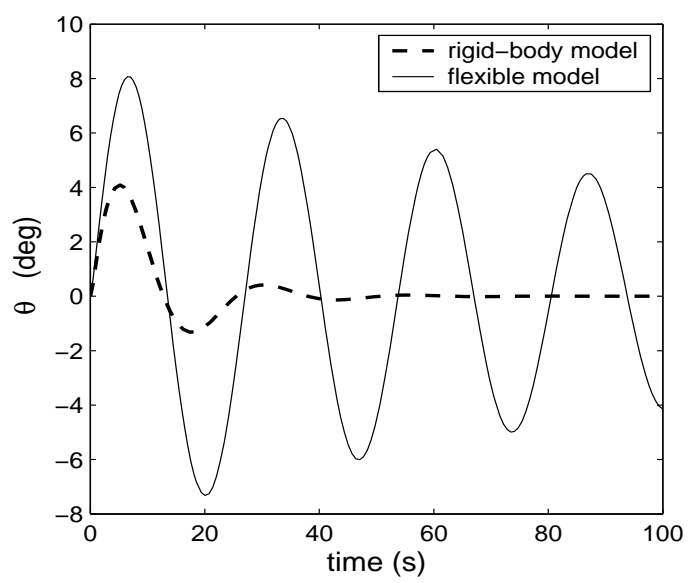

(c) At $86 \mathrm{~m} / \mathrm{s}$

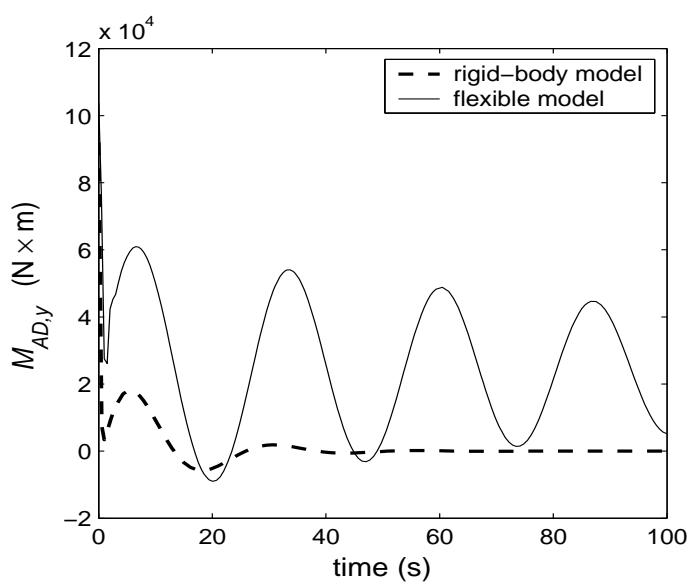

(e) At $86 \mathrm{~m} / \mathrm{s}$

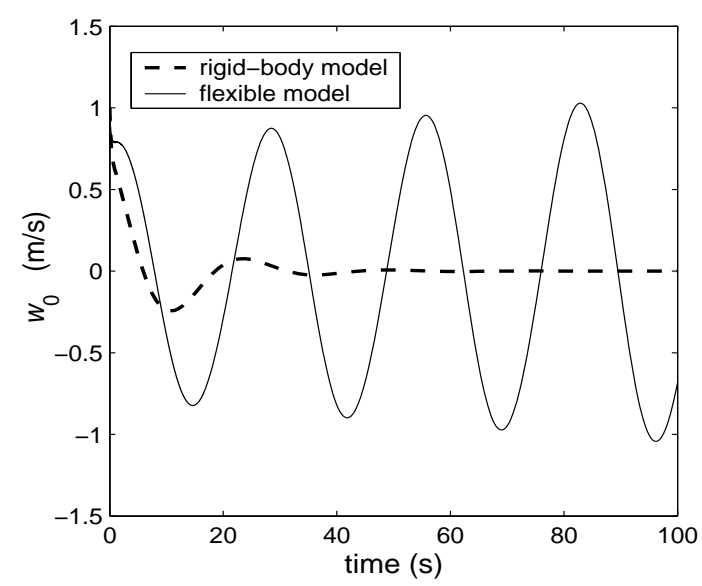

(b) At $90 \mathrm{~m} / \mathrm{s}$

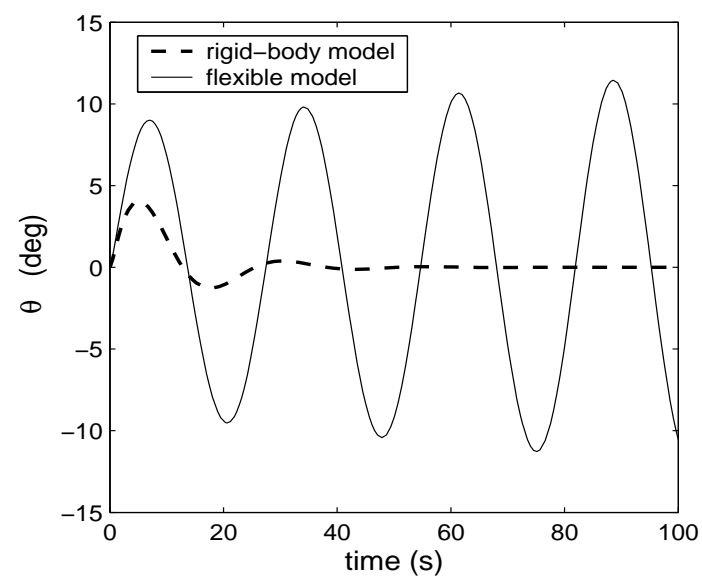

(d) At $90 \mathrm{~m} / \mathrm{s}$

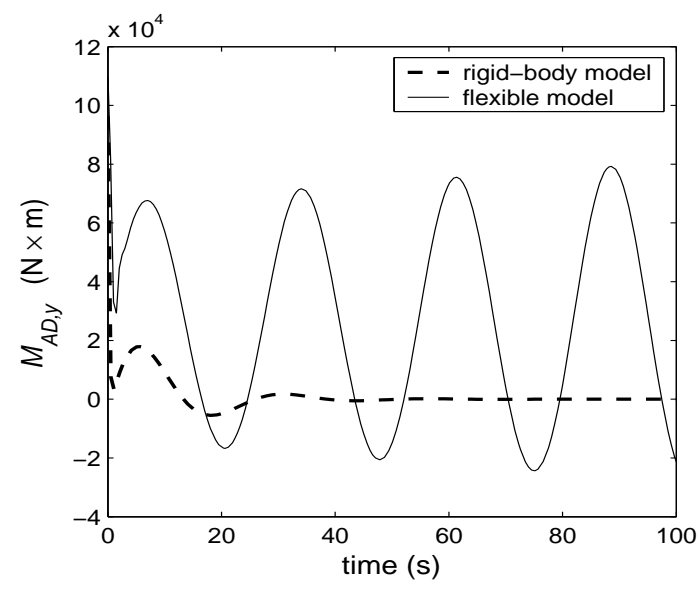

(f) At $90 \mathrm{~m} / \mathrm{s}$

Figure 6.6: Time histories of vertical speed, pitch angle and aerodynamic pitch moment at $86 \mathrm{~m} / \mathrm{s}$ and $90 \mathrm{~m} / \mathrm{s}$, given a disturbance of $\Delta w_{0}=1 \mathrm{~m} / \mathrm{s}$ 


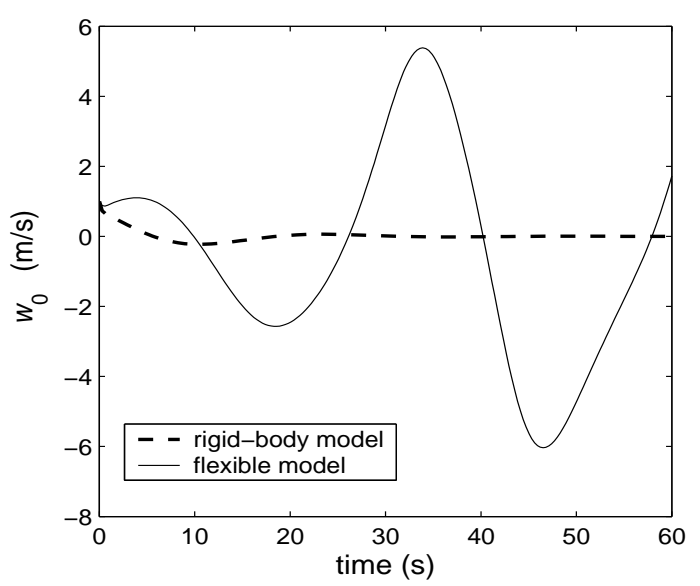

(a) At $105 \mathrm{~m} / \mathrm{s}$

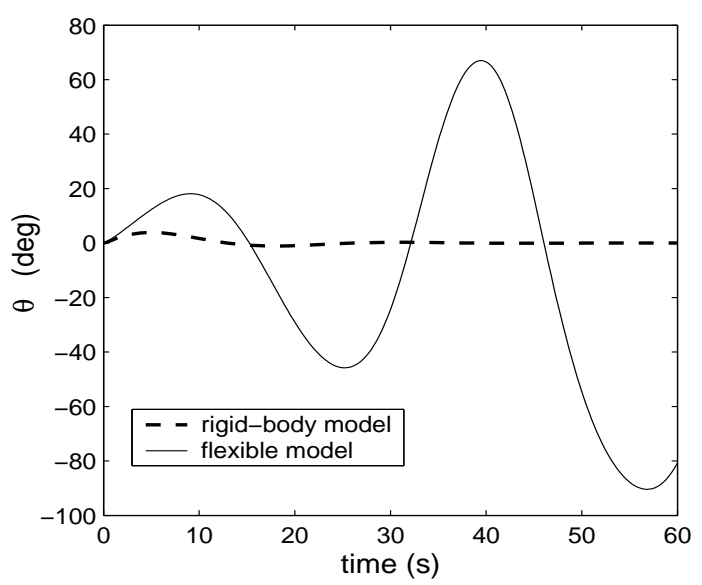

(c) At $105 \mathrm{~m} / \mathrm{s}$

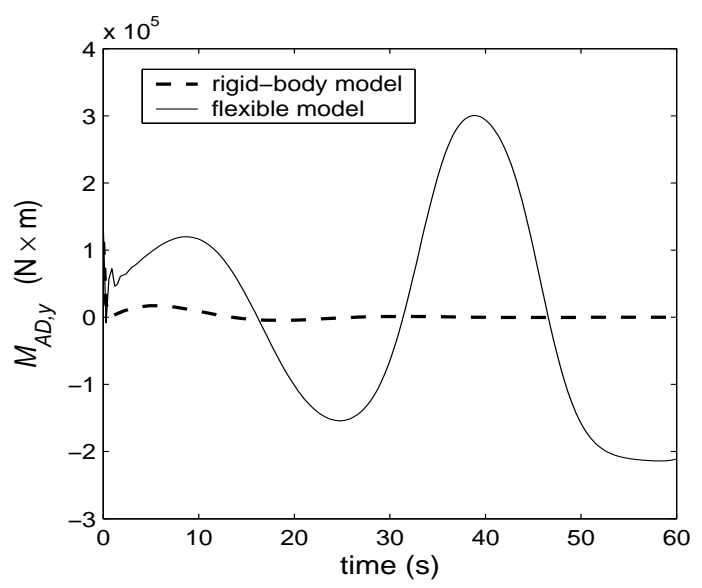

(e) At $105 \mathrm{~m} / \mathrm{s}$

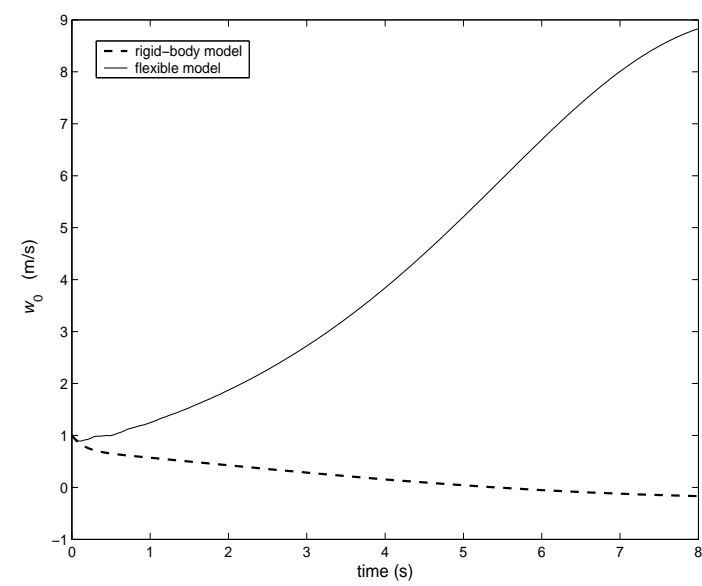

(b) At $125 \mathrm{~m} / \mathrm{s}$

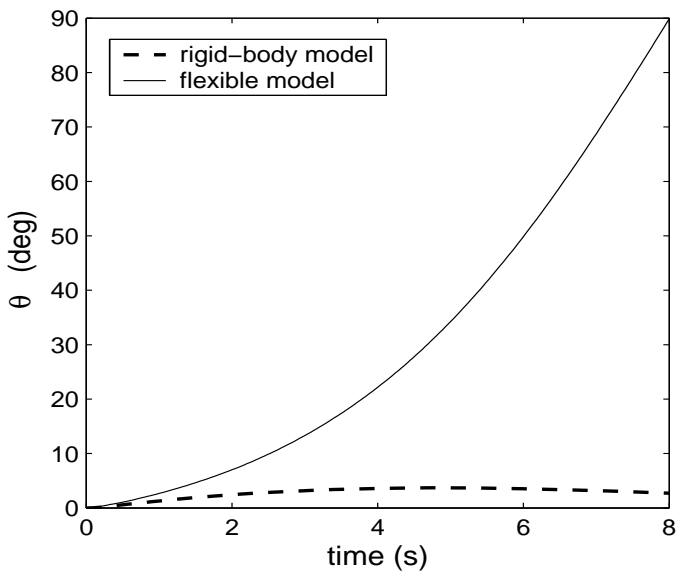

(d) At $125 \mathrm{~m} / \mathrm{s}$

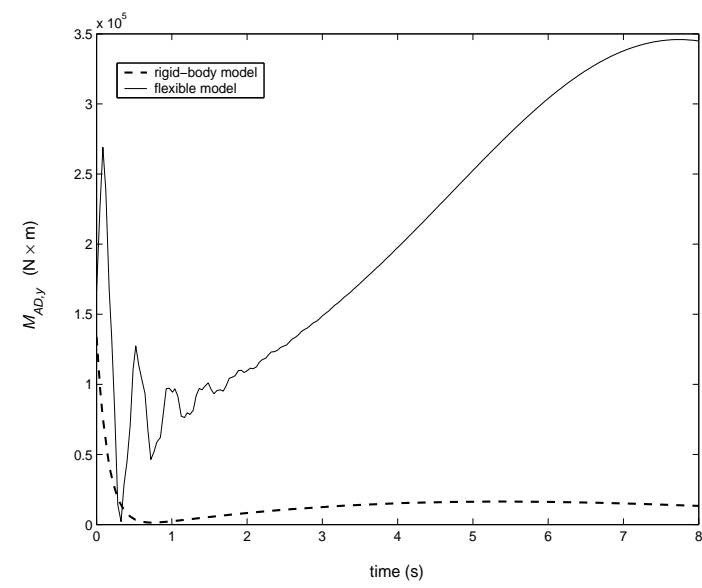

(f) At $125 \mathrm{~m} / \mathrm{s}$

Figure 6.7: Time histories of vertical speed, pitch angle and aerodynamic pitch moment at $105 \mathrm{~m} / \mathrm{s}$ and $125 \mathrm{~m} / \mathrm{s}$, given a disturbance of $\Delta w_{0}=1 \mathrm{~m} / \mathrm{s}$ 


\subsection{Influence of $E T$ on $u_{\text {unstable }}$}

The structural characteristics of airships depend on their envelope material, in addition to the geometry. Recently, very thin films have been proposed for stratospheric airships [77]. These materials were originally developed as part of NASA's Ultra Long Duration Balloon project $[77,117]$. It is possible to use such thin films for highaltitude LTA vehicles because the air density and wind speed, and the corresponding aerodynamic loads are relatively low in the stratosphere. Since these films provide much lower values of $E T$, we investigate the impact of ET on an airship's aeroelastic stability. For this purpose, the elastic modulus $E$ and thickness $T$ of the envelope of the Skyship-500 in Eq. (5.4) are changed to the following values:

$$
E=2.14 \times 10^{8} \mathrm{~Pa}, \quad T=0.048 \times 10^{-3} \mathrm{~m}
$$

These material properties are of a thin film employed for ultra long-duration balloons given in [117]. The resulting bending stiffness $E I$ is approximately $1 / 40$ of that used in the simulation examples presented in Sections 5.3.1 to 6.1.

Using the material properties in Eq. (6.1), the eigenvalues of the longitudinal and lateral modes are displayed in Figs. 6.8 and 6.9 respectively. The aeroelastic instability occurs at $u_{\text {unstable }}=16.6 \mathrm{~m} / \mathrm{s}$, however, now in the lateral motion and is associated with the first lateral rigid-body mode. This instability in the lateral motion is caused by the yaw Munk moment and by the decrease of the viscous effect and vertical fin force due to the bending deflection. The instability first occurs in the lateral plane but not in the longitudinal plane like was the case in Section 6.1, because the first lateral rigid-body mode is less stable than the third longitudinal rigid-body mode at the speed of $16.6 \mathrm{~m} / \mathrm{s}$. 

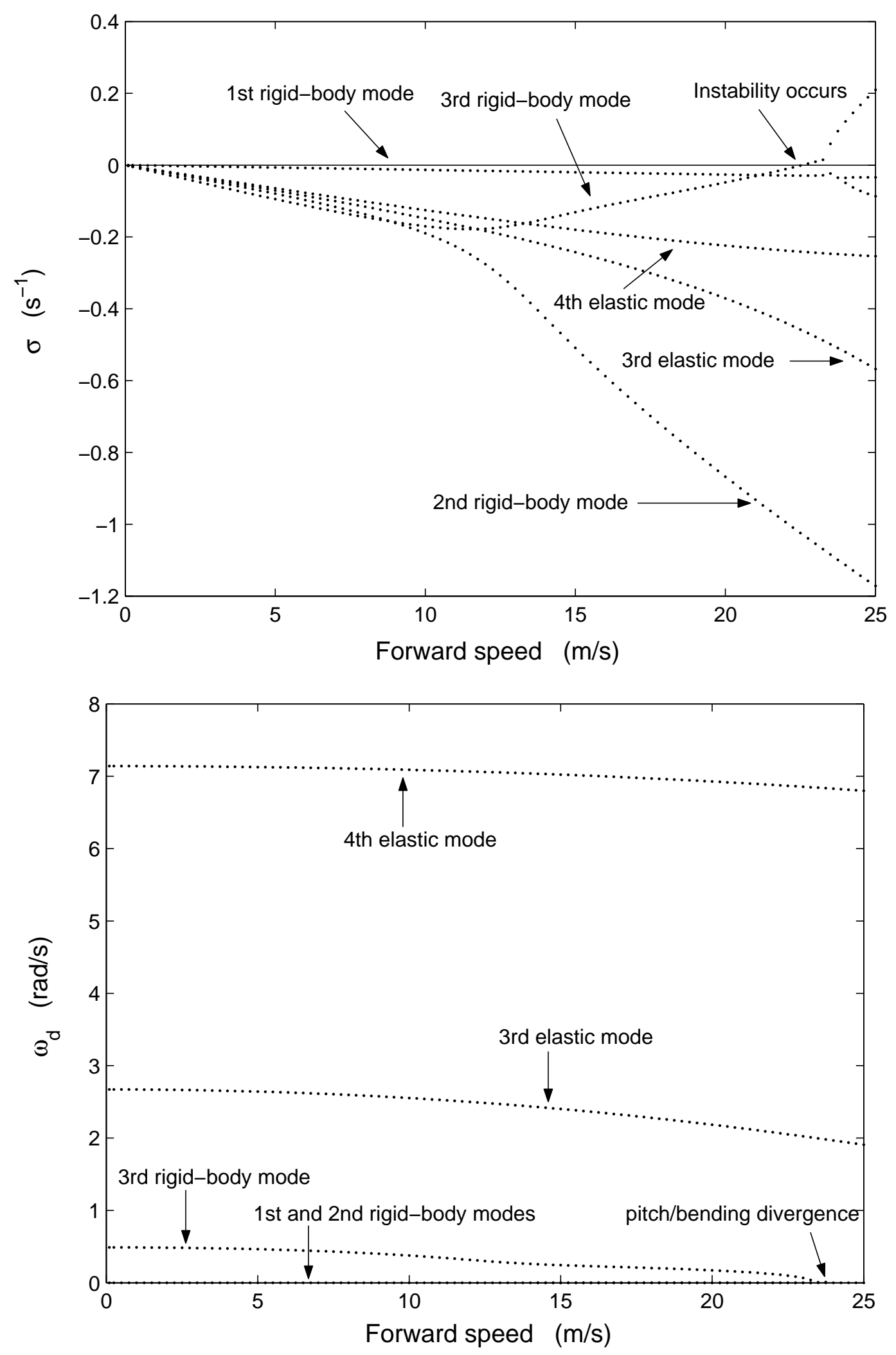

Figure 6.8: Eigenvalues of longitudinal modes from flexible-body dynamics, with material in Eq. (6.1) 

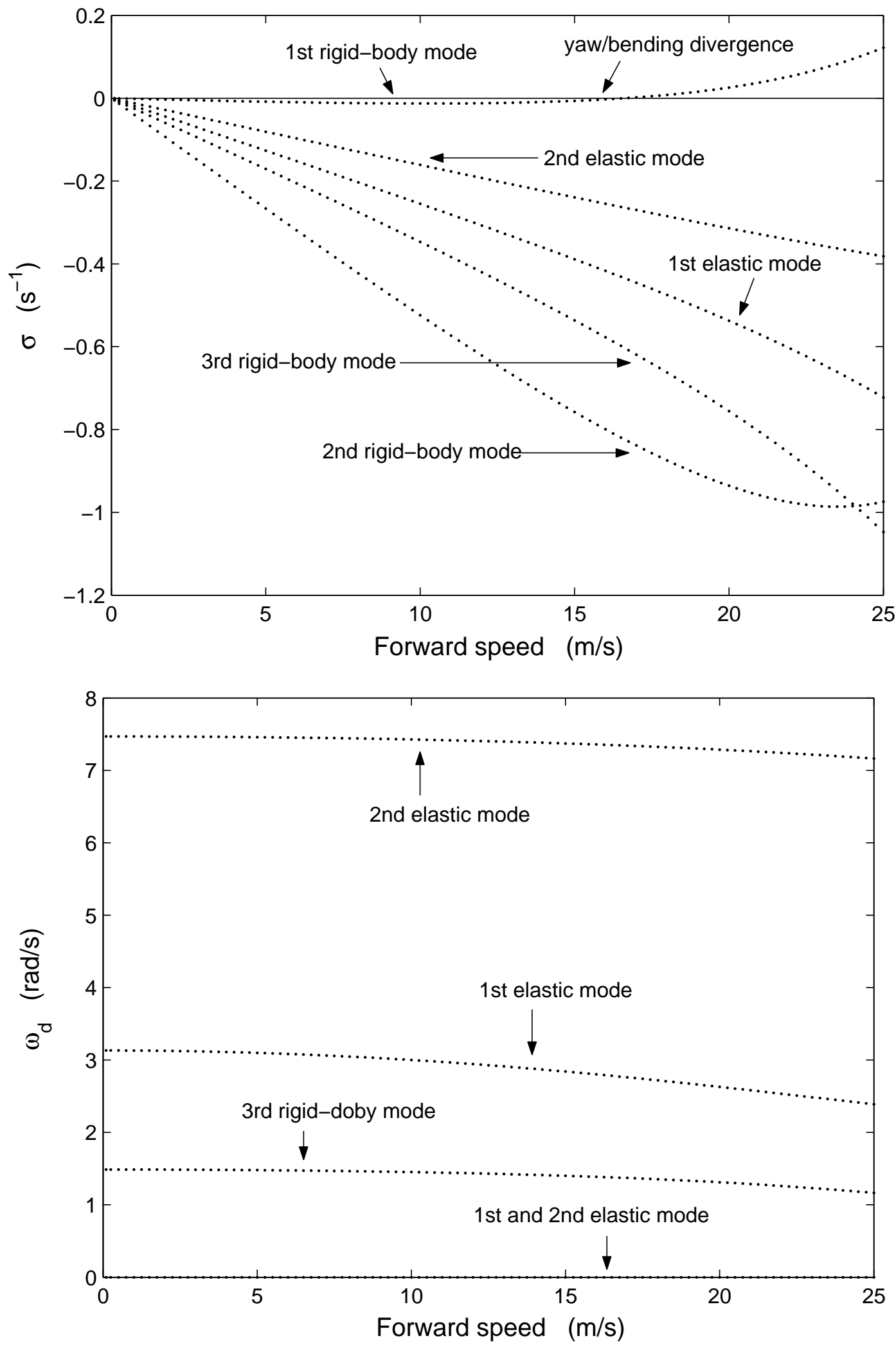

Figure 6.9: Eigenvalues of lateral modes from flexible-body dynamics, with material in Eq. (6.1) 


\subsection{Summary of the Aeroelastic Stability Analysis}

For fixed-wing aircraft, the divergence of a wing is caused by the negative aerodynamic stiffness, and flutter is generated from the coupling between bending and torsional deformations. However, the aeroelastic instability of an airship discussed here is due to the interaction between the aerodynamics, deformation and rigid-body motion. The instability results from the fact that the bending deflection reduces the aerodynamic effects that stabilize the airship against the Munk moment.

The aeroelastic stability also depends on the elastic modulus and thickness of the envelope. Using conventional materials, the aeroelastic instability will not occur for the Skyship-500. However, our analysis indicates that thin film materials discussed in Section 6.2 are not suitable for a 50-meter airship operating at low altitudes. 


\section{Chapter 7}

\section{Conclusions}

This thesis is devoted to the dynamics modeling and simulation of flexible airships, with a particular focus on a theoretical framework that unifies the flight dynamics, structural dynamics, aerostatics and aerodynamics.

The study began with a dynamics model of airships based on a rigid-body assumption. A comprehensive aerodynamic computational model was proposed, in which the aerodynamic effects were categorized into various terms based on different physical effects: the added-mass force, the viscous effect, the force on the fins, the force on the hull due to the fins, and the axial drag. A series of prediction methods were used for each of these aerodynamic terms. The most significant aerodynamic effect on an airship, the Munk moment in the added-mass terms, tends to destabilize the pitch and yaw rotations, while other aerodynamic forces normal to the centerline stabilize the airship. The numerical results of the added mass and moment of inertia, the steady-state aerodynamic force and moment, the time and frequency responses to control surface inputs, and the steady turn rates were compared to existing CFD, wind-tunnel and flight test data. The comparison showed that the present aerodynamic computational model can lead to reasonable prediction of the aerodynamic and dynamic characteristics of an airship. A linearized model was computed numerically 
to analyze the flight stability of the Skyship-500 airship. We found that all modes were stable based on a rigid-body model. Furthermore, a simplified 2-DOF dynamics model was developed to predict the steady turn rates, providing an accurate, effective and easy means to evaluate the turn performance of an airship.

With a thorough understanding of the aerodynamic effects on airships and the trust from validation based on the rigid-body model, we proceeded to formulate the dynamics model of flexible airships. The airship was modeled as a free-free EulerBernoulli beam and the bending deformations were represented by time-dependent generalized coordinates and time-independent shape functions. Once the velocity distribution of the flexible airship was obtained, the Lagrangian formulation was applied to derive the dynamics model that describes the elastic deformation and the rigidbody motion of the airship. The inertial, gravity, aerostatic and thrust forces were then incorporated, including the effects of structural flexibility. In order to capture the coupling between the aerodynamic forces and structural elasticity, the local velocity distribution on the deformed vehicle was used in the computation of various aerodynamic forces. Finally, the dynamics model of an elastic airship was represented by a single set of nonlinear ordinary differential equations, where the effects of flexibility on different forces were expressed in terms of generalized coordinates, generalized velocities, and shape function integrals.

A dynamics simulation program was developed in Matlab and used to analyze the dynamics characteristics of the Skyship-500 and the following simulation results were presented: the normal free-free mode shapes, shape function integrals, time and frequency responses to elevator and rudder inputs. From the simulation results, we observed that strong coupling exists between the roll rotation and the bending deflection in the lateral plane through the angular momentum coefficient matrix. The natural frequencies of the airship in the air are greatly affected by the generalized added-mass matrix, and are about 25-30\% lower than the natural frequencies in vac- 
uum. The effects of deformation become more obvious as the flight speed increases. However, generally speaking, the influence of flexibility on the rigid-body motion is relatively small for the Skyship-500 at its operating speeds.

The possibility of aeroelastic instability was investigated using the linearized dynamics model. The aeroelastic instability of an airship is associated with the interaction between the aerodynamics, deformation and rigid-body motion, because of the fact that the bending deflection reduces the aerodynamic effects that stabilize the airship against the Munk moment. As a result, some rigid-body modes can become unstable as the flight speed increases. The numerical results obtained from the stability analysis showed that the aeroelastic instability speeds are more than twice the operating speeds of the Skyship-500. The effects of the envelope's elastic modulus and thickness on the aeroelastic instability conditions were analyzed in order to investigate the possibility of employing a very thin film for the Skyship-500. The simulation results showed that the instability speed could be as low as $16.6 \mathrm{~m} / \mathrm{s}$. This indicated that such thin films cannot be used for the Skyship-500 at low altitude.

The present dynamics model provides insights into the interaction between the rigid-body motion, elastic deformation and aerodynamic forces of an airship. It can be extended to other airships to evaluate their dynamics behavior and their control systems. The results in this work have demonstrated that the structural deformation has small effects on the dynamics properties of the Skyship-500 airship. However, in order to reduce the weight of the envelope, very thin films have been proposed for airships. Since these films lead to much lower bending stiffness than the conventional airship envelopes, the effects of flexibility on these designs should be considered. 


\subsection{Recommendations for Future Research}

Several directions are suggested for future research based on the study presented in this dissertation:

- Although the Euler-Bernoulli beam model has been employed successfully to analyze the deflection behavior of inflated cylinders and also has been verified through experiments for several non-rigid airships, the structural deformation model should be further refined for flexible airships. Efforts should be made to model the effects of shear and torsional deformations of the hull, and the deformation in the cross section related to the membrane modes of the envelope.

- The wrinkling of the hull envelope has not been investigated in this work, but it has been demonstrated that the wrinkling can considerably influence the bending stiffness of inflated cylinders [82]. Prediction methods should be developed for the conditions under which wrinkling can occur on an airship, and the effects of wrinkling on the dynamics model should be investigated.

- Since the atmospheric turbulence can introduce high frequency disturbance to the system, it may excite structural vibration and cause considerable flexibility effects on the airship's dynamics. However, wind disturbances have not been considered in this work. To overcome this limitation, an accurate wind model should be incorporated into the dynamics model and simulation.

- The aeroelastic instability in Section 6.1 is associated with the rigid-body motion. Automatic control systems may be designed to improve the aeroelastic stability. To the author's knowledge, existing control systems for airships are based on a rigid-body dynamics model. To obtain better flight performance of very flexible airships such as those made of thin films mentioned in Section 6.2, one should develop new control techniques by considering the structural flexibility. 
- Simulation results in Section 6.2 have clearly shown that the thin films cannot be used for the Skyship-500 at low altitude. Efforts should be made to investigate the flexibility effects on the dynamics characteristics and the aeroelastic instability conditions of high-altitude airships made of these films.

- The comprehensive aerodynamics model in this work provides an effective way to predict the aerodynamic force and force distribution along the hull. However, for better understanding of the aerodynamics characteristics such as the pressure distribution over the hull surface, more complicated numerical approaches should be investigated in the aerodynamics computation.

- The thrust force calculation in this work is based on a simple proportional model, which produces reasonable airspeed results for the Skyship-500 in an acceleration test. However, the dynamics of the thruster may be an important issue for the dynamics analysis of smaller airships. A more detailed thruster model for airships is recommended.

- The actual C.G. location of an airship shifts during the flight due to the motion and inflation/deflation of the ballonets. The effects of ballonets should be incorporated into the dynamics model. 


\section{Bibliography}

[1] Gerken, L. C., Airships: History and Technology, American Scientific Corp., Chula Vista, CA, 1990, pp. 1-19.

[2] Khoury, G. and Gillett, J., Airship Technology, Cambridge University Press, Cambridge, UK, 1999, pp. 73-106, 141-209, 475-504.

[3] Elfes, A., Bueno, S. S., Bergerman, M., and Ramos, J. G., "A Semi-autonomous Robotics Airship for Environmental Monitoring Missions," Proceedings of the IEEE International Conference on Robotics and Automation, Vol. 4, Leuven, Belgium, May 1998, pp. 3449-3455.

[4] Wilson, J. R., "A New Era for Airships," Aerospace America, Vol. 42, No. 5, 2004, pp. 27-31.

[5] Hygounenc, E., Jung, I., Soueres, P., and Lacroix, S., "The Autonomous Blimp Project of LAAS-CNRS: Achievements in Flight Control and Terrain Mapping," The International Journal of Robotics Research, Vol. 23, No. 4-5, 2004, pp. 473-511.

[6] Kulczycki, E. A., Joshi, S. S., Hess, R. A., and Elfes, A., "Towards Controller Design for Autonomous Airship Using SLC and LQR Methods," AIAA Guidance, Navigation and Control Conference and Exhibit, Keystone, Colorado, August 21-24, 2006.

[7] Schmidt, D. K., "Modeling and Near-Space Stationkeeping Control of a Large HighAltitude Airship," Journal of Guidance, Control and Dynamics, Vol. 30, No. 2, 2007, pp. $540-547$. 
[8] Merriam-Webster, Merriam-Webster's Collegiate Dictionary, Merriam-Webster Inc., Springfield, MA, 11th ed., 2004.

[9] Etkin, B., Dynamics of Flight: Stability \& Control, Wiley, New York, 3rd ed., 1996, pp. 93-104, 156-159.

[10] Pamadi, B. N., Performance, Stability, Dynamics and Control of Airplanes, AIAA, Reston, VA, 2nd ed., 2004, pp. 361-391.

[11] Fossen, T. I., Guidance and Control of Ocean Vehicles, Wiley, New York, 1998, pp. $5-42$.

[12] Tischler, M. B., Ringland, R. R., and Jex, H. R., "Heavy Airship Dynamics," Journal of Aircraft, Vol. 20, No. 5, 1983, pp. 425-433.

[13] Tischler, M. B., Jex, H. R., and Ringland, R. F., "Simulation of Heavy Lift Airship Dynamics over Large Ranges of Incidence and Speed," AIAA Lighter-Than-Air Systems Technology Conference, Annapolis, MD, July 8-10, 1981, pp. 96-115.

[14] Tischler, M. B. and Jex, H. R., "Effects of Atmospheric Turbulence on a Quadrotor Heavy Lift Airship," Journal of Aircraft, Vol. 20, No. 12, 1983, pp. 1050-1057.

[15] Amann, J. H., "A Comparison of a Nonlinear Flight Dynamic Simulation of an Airship with Flight Test Results," 7th AIAA Lighter-Than-Air Technology Conference, Monterey, CA, August 17-19, 1987, pp. 78-86.

[16] Jones, S. P. and DeLaurier, J. D., "Aerodynamic Estimation Techniques for Aerostats and Airships," Journal of Aircraft, Vol. 20, No. 2, 1983, pp. 120-126.

[17] Jex, H. R. and Gelhausen, P., "Pre- and Post-Flight-Test Models versus Measured Skyship-500 Control Responses," 7th AIAA Lighter-Than-Air Technology Conference, Monterey, CA, August 17-19, 1987, pp. 87-97.

[18] Thomasson, P. G., "Equations of Motion of a Vehicle in a Moving Fluid," Journal of Aircraft, Vol. 37, No. 4, 2000, pp. 630-639. 
[19] Azinheira, J. R., de Paiva, E. C., and Bueno, S. S., "Influence of Wind Speed on Airship Dynamics," Journal of Guidance, Control and Dynamics, Vol. 25, No. 6, 2002, pp. $1116-1124$.

[20] Yamasaki, T. and Goto, N., "Identification of Blimp Dynamics via Flight Tests," Transactions of the Japan Society for Aeronautical and Space Sciences, Vol. 46, No. 153, 2003, pp. 195-205.

[21] Jones, R., "The Application of the Results of Experiments on Model Airships to FullScale Turning," ARC RM-716, 1920.

[22] Jones, R. and Bell, A. H., "Experiments on a Model of the Airship R.101 with Applications to Determine the Steady Motion of the Airship," ARC RM-1400, 1931.

[23] Cook, M. V., Lipscombe, J. M., and Goineau, F., "Analysis of the Stability Modes of the Non-Rigid Airship," The Aeronautical Journal, Vol. 104, No. 1036, 2000, pp. 279 290.

[24] Munk, M. M., "The Aerodynamic Forces on Airship Hulls," NACA TR-184, 1924.

[25] Lamb, H., Hydrodynamics, Dover, New York, 6th ed., 1945, pp. 160-201.

[26] von Karman, T., "Calculation of Presure Distribution on Airship Hulls," NACA TM$574,1930$.

[27] Upson, R. H. and Klikoff, W. A., "Application of Practical Hydrodynamics of Airship Design," NACA TR-405, 1933.

[28] Allen, H. J. and Perkins, E. W., "Estimation of the Forces and Moments Acting on Inclined Bodies of Revolution of High Fineness Ratio,” NACA RM-a9i26, 1949.

[29] Allen, H. J. and Perkins, E. W., "A Study of Effects of Viscosity on Flow over Slender Inclined Bodies of Revolution," NACA TR-1048, 1951.

[30] Hopkins, E. J., "A Semi-empirical Method for Calculating the Pitching Moment of Bodies of Revolution at Low Mach Numbers," NACA RM-A51C14, 1951. 
[31] Lawrence, H. R. and Flax, A. H., "Wing-Body Interference at Subsonic and Supersonic Speeds - Survey and New Developments," Journal of the Aeronautical Sciences, Vol. 21, No. 5, 1954, pp. 289-324.

[32] Pitts, W. C., Nielsen, J. N., and Kaattari, G. E., "Lift and Center of Pressure of WingBody-Tail Combinations at Subsonic, Transonic and Supersonic Speeds," NACA TR1307, 1957.

[33] Wong, K. Y., ZhiYung, L., and DeLaurier, J., "An Application of Source-Panel and Vortex Methods for Aerodynamic Solutions of Airship Configurations," 6th AIAA Lighter-Than-Air Technology Conference, Norfolk, VA, June 26-28, 1985, pp. 78-83.

[34] Lutz, T., Leinhos, D., Jakobi, A., and Wagner, S., "Theoretical Investigations of the Flowfield of Airships with a Stern Propeller," Proceedings International Airship Convention and Exhibition, Bedford, UK, July 5-7, 1996.

[35] Lutz, T., Funk, P., Jakobi, A., and Wagner, S., "Aerodynamic Investigations on Inclined Airship Bodies," 2nd International Airship Convention and Exhibition, Bedford, UK, July 26-28, 1998.

[36] Jakobi, A., Lutz, T., and Wagner, S., "Aerodynamic Investigations on Inclined Airship Bodies - Boundary Layer Calculation Method," 3rd International Airship Convention and Exhibition, Friedrichshafen, Germany, July 1-5, 2000.

[37] Jakobi, A., Funk, P., Lutz, T., and Wagner, S., "Modelling of Airship Wakes Applying Higher Order Panel Elements," 14th AIAA Lighter-Than-Air Technical Committee Convention and Exhibition, Akron, OH, July 15-19, 2001.

[38] Lutz, T., Funk, P., Jakobi, A., and Wagner, S., "Calculation of the Propulsive Efficiency for Airships with Stern Thruster," 14th AIAA Lighter-Than-Air Technical Committee Convention and Exhibition, Akron, OH, July 15-19, 2001.

[39] Lutz, T., Fund, P., Jakobi, A., and Wagner, S., "Summary of Aerodynamic Studies on the Lotte Airship," Proceeding of the 4th International Airship Convention and Exhibition, Cambridge, UK, July 28-31, 2002. 
[40] Lutz, T. and Wagner, S., "Drag Reduction and Shape Optimization of Airship Bodies," Journal of Aircraft, Vol. 35, No. 3, 1998, pp. 345-351.

[41] Nejati, V. and Matsuuchi, K., "Aerodynamics Design and Genetic Algorithms for Optimization of Airship Bodies," JSME International Journal, Series B: Fluids and Thermal Engineering,, Vol. 46, No. 4, 2003, pp. 610-617.

[42] Wang, X.-L. and Shan, X.-X., "Shape Optimization of Stratospere Airship," Journal of Aircraft, Vol. 43, No. 1, 2006, pp. 283-287.

[43] Jones, R., "The Aerodynamical Characteristics of the Airship as Deduced from Experiments on Models, with Application to Motion in a Horizaontal Plane," Journal of Royal Aeronautical Society, Vol. 28, No. 158, 1924, pp. 88-150.

[44] Curtiss, H. C., Hazen, D. C., and Putman, W. F., "LTA Aerodynamic Data Revised," Journal of Aircraft, Vol. 13, No. 11, 1976, pp. 835-844.

[45] Freeman, H. B., "Measurements of Flow in the Boundary Layer of a 1/40-Scale Model of the U.S. Airship 'Akron'," NACA TR-430, 1933.

[46] Freeman, H. B., "Force Measurements on a 1/40-Scale Model of the U.S. Airship Akron," NACA TR-432, 1933.

[47] Freeman, H. B., "Pressure Distribution Measurements on the Hull and Fins of a 1/40Scale Model of the U. S. Airship Akron," NACA TR-443, 1934.

[48] Silverstein, A. and Gulick, B. G., "Ground-handling Forces on a 1/40-Scale Model of the U. S. Airship 'Akron'," NACA TR-566, 1937.

[49] McHugh, J. G., "Pressure-distribution Measurements at Large Angles of Pitch on Fins of Different Span-Chord Ratio on a 1/40-scale Model of the U. S. Airship 'Akron'," NACA TR-604, 1937.

[50] Zahm, A. F., "Air Forces, Moments and Damping on Model of Fleet Airship Shenandoah," NACA TR-215, 1926. 
[51] Jones, R., Williams, D. H., and Bell, A. H., "Experiments on a Model of the Airship R.29," ARC RM-714, 1920.

[52] Jones, R. and Bell, A. H., "Experiments on a Model of the Airship R.101," ARC RM-1168, 1926.

[53] Pannel, J. R. and Jones, R., "Experiments on a Model of the German Rigid Airship L33," ARC RM-361, 1917.

[54] Funk, P., Jakobi, A., Lutz, T., and Wagner, S., "Experimental Investigations in the Tail Region of an Airship Configuration," 14th AIAA Lighter-Than-Air Technical Committee Convention and Exhibition, Akron, OH, July 15-19, 2001.

[55] Pannell, J. R. and Frazer, R. A., "Account of Some Experiments on Rigid Airship R.26," ARC RM-674, 1920.

[56] Pannell, J. R. and Bell, A. H., "Experiments on Rigid Airship R.29," ARC RM-675, 1920.

[57] Pannell, J. R. and Frazer, R. A., "Experiments on Rigid Airship R.33," ARC RM-668, 1919.

[58] DeFrance, S. J., "Flight Tests on U.S.S. Los Angeles. Part I, Full Scale Pressure Distribution Investigation," NACA TR-324, 1930.

[59] Burgess, C. P., "Flight Tests on U.S.S. Los Angeles. Part II, Stress and Strength Determination," NACA TR-325, 1930.

[60] Thompson, F. L., "Full-Scale Turning Characteristics of the U. S. S. Los Angeles," NACA TR-333, 1930.

[61] Bailey, D. B., "Patrol Airship Concept Evaluation (PACE): Final Report," NADC85019-60, 1985.

[62] Jex, H. R. and Gelhausen, P., "Control Response Measurements of the Skyship-500 Airship," 6th AIAA Lighter-Than-Air Technology Conference, Norfolk, VA, June 2628, 1985, pp. 130-141. 
[63] Kornienko, A., System Identification Approach for Determining Flight Dynamical Characteristics of an Airship from Flight Data, Ph.D. thesis, Universität Stuttgart, August 2006.

[64] Burgess, C. P., "The Strength of Rigid Airships," Journal of Royal Aeronautical Society, Vol. 28, No. 162, 1924, pp. 327-448.

[65] Ebner, H., "The Present Status of Airship Construction, Especially of Airship Framing Construction," NACA TR-872, 1937.

[66] Herrera, E., "The R-38 Catastrophe and the Mechanics of Rigid Airship Construction," NACA TM-105, 1922.

[67] Evans, J. R. and DeLaurier, J. D., "The Shenandoah Flies Again: A Computer Simulation," AIAA Lighter-Than-Air Systems Technology Conference, Annapolis, MD, July 8-10, 1981, pp. 62-73.

[68] Burgess, C. P., Airship Design, The Ronald Press Company, 1927, pp. 109-152.

[69] Hass, R. and Dietzius, A., "The Stretching of the Fabric and the Deformation of the Envelope in Nonrigid Balloons," NACA TR-16, 1917.

[70] Hunt, J. D., "Structural Analysis of Aerostat Flexible Structure by the Finite Element Method," Journal of Aircraft, Vol. 19, No. 9, 1982, pp. 674-678.

[71] Hunt, J. D., "Structural Analysis of the Light Weight Hard Nose on the 71M Aerostat," 10th AIAA Lighter-Than-Air Systems Technical Conference, Scottsdale, AZ, September 14-16, 1993.

[72] Witherow, R. G., "Structural Design Features of a 250,000 $\mathrm{ft}^{3}$ Tether Aerostat," 7th AFCRL Scientific Balloon Symposium, September 1972.

[73] Amiryants, G. A., Grigorive, V. D., Ishmuratov, F. Z., Franz, A., d'Henin, E., and Kaempf, B., "Investigations of Airship Aeroelasticity," 23rd International Congress of Aerospace Sciences, Toronto, Canada, 2002. 
[74] Bessert, N. and Frederich, O., "Nonlinear Airship Aeroelasticity," Journal of Fluids and Structures, Vol. 21, 2005, pp. 731-742.

[75] Omari, K., Schall, E., Koobus, B., and Dervieux, A., "Inviscid Flow Calculation Around a Flexible Airship," 8th Conference of Applied Mathematics and Statistics, Jaca, Spain, September 15-18, 2004.

[76] Bennaceur, S., Azouz, N., and Boukraa, D., "An Efficient Modelling of Flexible Airships," 8th Biennial ASME Conference on Engineering Systems Design and Analysis, Torino, Italy, July 4-7, 2006, pp. 573-582.

[77] Smith, M. S. and Rainwater, E. L., "Applications of Scientific Ballooning Technology to High Altitude Airships," 3rd AIAA Annual Aviation Technology, Integration, and Operations Technical Forum, Denver, CO, November 17-19, 2003.

[78] Jenkins, C. H. and Leonard, J. W., "Nonlinear Dynamic Response of Membranes: State of the Art," Applied Mechanics Review, Vol. 44, No. 7, 1991, pp. 319-328.

[79] Jenkins, C. H. and Leonard, J. W., "Nonlinear Dynamic Response of Membranes: State of the Art - update," Applied Mechanics Review, Vol. 49, No. 10, 1996, pp. S41S48.

[80] Fang, H. and Lou, M. C., "Analytical Characterization of Space Inflatable Structures - an Overview," 40th AIAA/ASME/ASCE/AHS/ASC Structures, Structural Dynamics, and Materials Conference and Exhibit, St. Louis, MO, April 12-15, 1999.

[81] Ruggiero, E. J., Jha, A., Park, G., and Inman, D. J., "A Literature Review of UltraLight and Inflated Toroidal Satellite Components," The Shock and Vibration Digest, Vol. 35, No. 3, 2003, pp. 171-181.

[82] Main, J. A., Perterson, S. W., and Strauss, A. M., "Load-Deflection Behavior of SpaceBased Inflatable Fabric Beams," Journal of Aerospace Engineering, Vol. 7, No. 2, 1994, pp. 225-238. 
[83] Main, J. A., Carlin, R. A., Garcia, E., Perterson, S. W., and Strauss, A. M., "Dynamics Analysis of Space-Based Inflated Beam Structures," Journal of Acoustical Society of America, Vol. 97, No. 2, 1995, pp. 1035-1045.

[84] Rybski, M., Shoham, M., and Grossman, G., "Robotic Manipulators Based on Inflatable Structures," Robotics \& Computer-Integrated Manufacturing, Vol. 12, No. 1, 1996, pp. 111-120.

[85] Suhey, J. D., Kim, N. H., and Niezrecki, C., "Numerical Modeling and Design of Inflatable Structures - Application to Open-Ocean-Aquaculture Cages," Aquacultural Engineering, Vol. 33, 2005, pp. 285-303.

[86] Young, L. G., Ramanathan, S., Hu, J., and Pai, P. F., "Numerical and Experimental Dynamic Characteristics of Thin-Film Membranes," International Journal of Solids and Structures, Vol. 42, 2005, pp. 3001-3025.

[87] Hodges, D. H. and Pierce, G. A., Introduction to Structural Dynamics and Aeroelasticity, Cambridge Universtiy Press, New York, 2002, pp. 1-4, 80-130.

[88] Fung, Y. C., An Introduction to the Theory of Aeroelasticity, Dover, New York, 1993, pp. 81-113, 186-244.

[89] Dowell, E. H. D., Curtiss, H. C., and Scanlan, R. H., A Modern Course in Aeroelasticity, Kluwer Academic Publishers, Dordrecht, Netherlands, 2nd ed., 1989, pp. 3-46.

[90] Bisplinghoff, R. L. and Ashley, H., Principles of Aeroelasticity, Dover, New York, 1975, pp. 281-329, 450-486.

[91] Waszak, M. R. and Schmidt, D. K., "Flight Dynamics of Aeroelastic Vehicles," Journal of Aircraft, Vol. 25, No. 6, 1998, pp. 563-571.

[92] Schmidt, D. K., "Modeling and Simulation of Flexible Flight Vehicles," Journal of Guidance, Control and Dynamics, Vol. 24, No. 3, 2001, pp. 539-546.

[93] Meirovitch, L. and Tuzcu, I., "Integrated Approach to the Dynamics and Control of Maneuvering Flexible Aircraft," NASA CR-211748, 2003. 
[94] Meirovitch, L. and Tuzcu, I., "Unified Theory for the Dynamics and Control of Maneuvering Flexible Aircraft," AIAA Journal, Vol. 42, No. 4, 2004, pp. 714-727.

[95] Bolender, M. A. and Doman, D. B., "A Non-Linear Model for the Longitudinal Dynamics of a Hypersonic Air-breathing Vehicle," AIAA Guidance, Navigation, and Control Conference and Exhibition, San Francisco, CA, 2005.

[96] Platus, D. H., "Aeroelastic Stability of Slender, Spinning Missiles," Journal of Guidance, Control and Dynamics, Vol. 15, No. 1, 1992, pp. 141-151.

[97] Patil, M. J. and Hodges, D. H., "Flight Dynamics of Highly Flexible Flying Wings," Journal of Aircraft, Vol. 43, No. 6, 2006, pp. 1790-1798.

[98] Cesnik, C. and Su, W., "Nonlinear Aeroelastic Modeling and Analysis of Fully Flexible Aircraft," 46th AIAA/ASME/ASCE/AHS/ASC Structures, Structural Dynamics and Materials Conference, Austin, Texas, April 18-21, 2005.

[99] Lewis, F. M., "The Inertia of the Water Surrounding a Vibrating Ship," Transactions of the Society of Naval Architects and Marine Engineers, Vol. 37, 1929, pp. 1-20.

[100] Macagno, E. O. and Landweber, L., "Irrotational Motion of the Liquid Surrounding a Vibrating Ellipsoid of Revolution," Journal of Ship Research, Vol. 2, No. 1, 1958, pp. 37-49.

[101] Armand, J.-L. and Orsero, P., "A Method for Evaluating the Hydrodynamic Added Mass in Ship Hull Vibrations," Transactions of the Society of Naval Architects and Marine Engineers, Vol. 87, 1979, pp. 99-120.

[102] Vorus, W. S. and Hylarides, S., "Hydrodynamic Added-Mass Matrix of Vibrating Ship Based on a Distribution of Hull Surface Sources," Transactions of the Society of Naval Architects and Marine Engineers, Vol. 89, 1981, pp. 397-416.

[103] Newman, J. N., Marine Hydrodynamics, MIT press, Cambridge, MA, 1977, pp. 132149.

[104] Nielsen, J. N., Missile Aerodynamics, AIAA, Cambridge, MA, 1988, pp. 363-394. 
[105] Blevins, R. D., Formulas for Natural Frequency and Mode Shape, Robert E. Krieger Publishing Company, New York, 1979, p. 405.

[106] Meyerhoff, W. K., "Added Masses of Thin Rectangular Plates Calculated from Potential Theory," Journal of Ship Research, Vol. 14, No. 2, 1970, pp. 100-111.

[107] Finck, R. D., USAF Stability and Control DATCOM, Flight Control Division, Air Force Dynamics Laboratory, Wright-Patterson Air Force Base, OH, 1978, Section 4.1, 4.2 and 6.1.4.

[108] McCormick, B. W., Aerodynamics, Aeronautics and Flight Mechanics, Wiley, New York, 2nd ed., 1995, pp. 99-116, 160-165.

[109] von Karman, T. and Burgers, J. M., "General Aerodynamic Theory - Perfect Fluids," Aerodynamic Theory, edited by W. F. Durand, Vol. II, Dover, New York, 1943, p. 153.

[110] Blanger, P., Control Engineering: A Modern Approach, Saunders College Publishing, Orlando, FL, 1995, pp. 24-32.

[111] Lambert, C. and Nahon, M., "Stability Analysis of a Tethered Aerostat," Journal of Aircraft, Vol. 40, No. 4, 2003, pp. 705-715.

[112] Nikravesh, P. E., "Understanding Mean-Axis Conditions as Floating Reference Frames," Advances in Computational Multibody Systems, edited by J. A. C. Ambrosio, Springer, Netherlands, 2005, pp. 185-203.

[113] McTavish, D. J. and Davidson, K., "Practical Large-Motion Modeling of Geometrically Complex Flexible Vehicles," 47th AIAA/ASME/ASCE/AHS/ASC Structures, Structural Dynamics, and Materials Conference, Newport, Rhode Island, May 1-4, 2006.

[114] Damaren, C. and Sharf, I., "Simulation of Flexible-Link Manipulators with Inertial and Geometric Nonlinearities," Journal of Dynamic Systems, Measurement and Control, Vol. 117, 1995, pp. 74-87.

[115] Shampine, L. F. and Reichelt, M. W., "The MATLAB ODE Suite," SIAM Journal on Scientific Computating, Vol. 18, 1997, pp. 1-22. 
[116] Nicholson, J. W., "The Lateral Vibration of Bars of Variable Section," Proceedings of the Royal Society of London. Series A, Containing Papers of a Mathematical and Physical Character, Vol. 654, 1917, pp. 506-519.

[117] Pagitz, M. and Pellegrino, S., "Shape Optimization of 'Pumpkin' Balloons," AIAA Balloon Systems Conference, Williamsburg, VA, May 21-24, 2007. 